\title{
Cutting the Gordian knot of excited-state modeling in complex environments
}

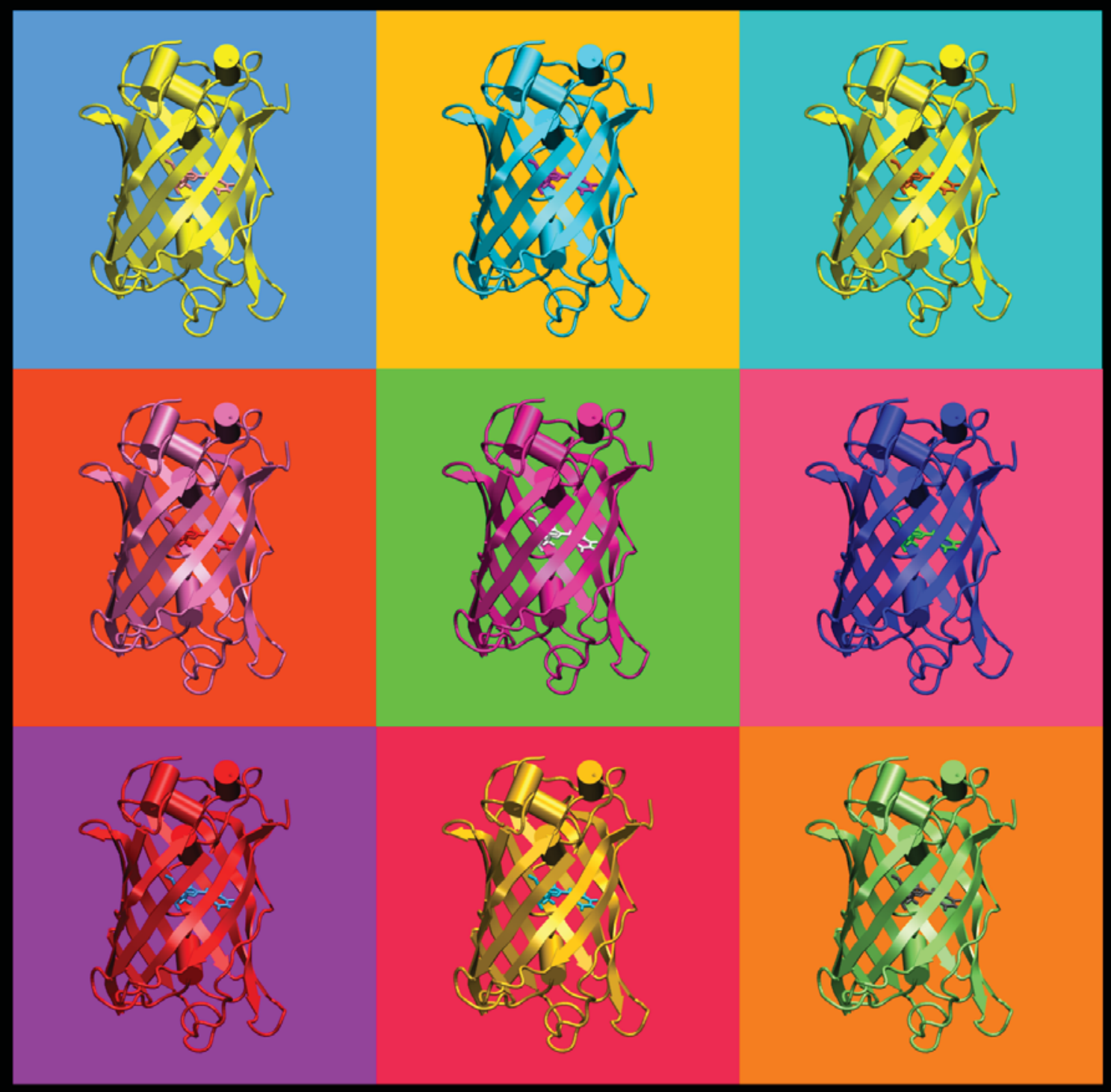

Csaba Daday 
CUTTING THE GORDIAN KNOT OF EXCITED-STATE MODELING IN COMPLEX ENVIRONMENTS

Csaba Daday 
Promotion committee:

Prof. dr. ir. J. W. M. Hilgenkamp University of Twente, Chairman

Prof. dr. ir. J. W. M. Hilgenkamp University of Twente, Secretary

Prof. dr. C. Filippi

University of Twente, Supervisor

Prof. dr. W. J. Briels

University of Twente

Dr. C. Blum

University of Twente

Prof. dr. L. Visscher

VU University Amsterdam

Prof. dr. J. Neugebauer

Münster University

Dr. A. Sinicropi

University of Siena

C. Daday

Cutting the Gordian knot of excited-state modeling in complex environments

Ph.D. Thesis, University of Twente, Enschede

ISBN: 978-90-365-3933-3

Copyright $(2015$ by C. Daday

No part of this work may be reproduced by print, photocopy or any other means without the permission in writing from the author.

DOI: $10.3990 / 1.9789036539333$

Online version: http://dx.doi.org/10.3990/1.9789036539333

Csaba Daday

dadaycs@gmail.com

Printed by: Gildeprint Drukkerijen - www.gildeprint.nl 


\section{CUTTING THE GORDIAN KNOT OF EXCITED-STATE MODELING IN COMPLEX ENVIRONMENTS}

\section{DISSERTATION}

to obtain

the degree of doctor at the University of Twente, on the authority of the rector magnificus,

Prof. dr. H. Brinksma, on account of the decision of the graduation committee,

to be publicly defended on Friday $18^{\text {th }}$ of September 2015 at $14: 45$

by

\section{Csaba Daday}

born on June 16, 1987

in Oradea, Romania 
This doctoral dissertation is approved by:

Prof. dr. C. Filippi 
Nature isn't classical, dammit, and if you want to make a simulation of nature, you'd better make it quantum mechanical, and by golly it's a wonderful problem, because it doesn't look so easy.

Richard Feynman 


\section{Contents}

1 Introduction 1

1.1 GFP and its mutants . . . . . . . . . . . . . . 1

1.1.1 Structure and photocycle of GFP . . . . . . . . . . . 3

1.2 Complex environments and multiscale methods: Point charges and

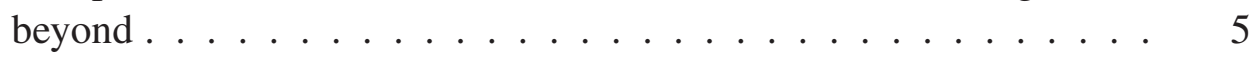

1.3 This thesis ........................... 9

2 Theoretical Methods 17

2.1 Introduction . . . . . . . . . . . . . . . . 17

2.2 Quantum Mechanical Calculations . . . . . . . . . . . . 17

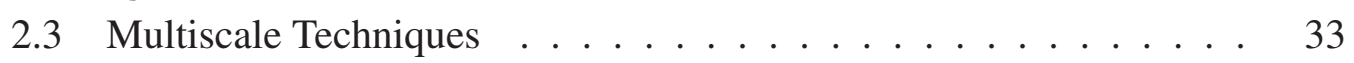

2.4 Computational Details . . . . . . . . . . . . . 39

3 State-Specific Embedding Potentials for Excitation-Energy Calculations 47

3.1 Introduction . . . . . . . . . . . . . . . . . . . . . . . . . 47

3.2 Theory ............................... 49

3.3 Computational details . . . . . . . . . . . . . . . . . 56

3.4 Results . . . . . . . . . . . . . . . . . . . . . . . . . . . . . . .

3.5 Conclusions ......................... 71

4 WF/DFT Embedding for Excited States: Which Wavefunctions, which $\begin{array}{ll}\text { Densities? } & 81\end{array}$

4.1 Introduction . . . . . . . . . . . . . . . . . 81

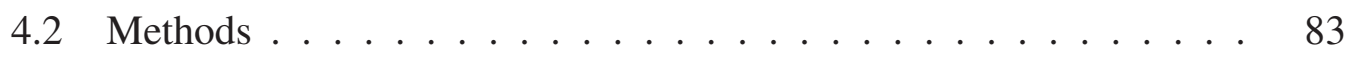

4.3 Computational details . . . . . . . . . . . . . . . . . . . . . . . . . . . . . . . . . . 87

4.4 Results . . . . . . . . . . . . . . . . . . . . . . . 89

4.5 Conclusions . . . . . . . . . . . . . . . 101

5 Multiscale excited-state modeling of GFP: Solving a polarizing issue 111

5.1 Introduction . . . . . . . . . . . . . . . . . 112

5.2 Computational details . . . . . . . . . . . . . . . . . . . . . . . . . . . . . . . 114

5.3 Results . . . . . . . . . . . . . . . . . . . . . 117

5.4 Discussion and conclusions . . . . . . . . . . . . . 136 
6 Wavefunction embedding using exact Freeze\&Thaw cycles 149

6.1 Theory . . . . . . . . . . . . . . . . . . . . . . 149

6.2 Computational details . . . . . . . . . . . . . . . . . 154

6.3 Results . . . . . . . . . . . . . . . . . . . . 155

6.4 Conclusions . . . . . . . . . . . . . . . . . 160

7 Full CI excitations of ethene and butadiene: Resolution of an ancient question 163

7.1 Introduction . . . . . . . . . . . . . . 163

7.2 The FCIQMC method . . . . . . . . . . . . . . . . . 165

7.3 Computational details . . . . . . . . . . . . . . . . 167

7.4 Results . . . . . . . . . . . . . . . . . . . . 170

7.5 Conclusions ......................... 183

$\begin{array}{lr}\text { List of publications } & 190\end{array}$

$\begin{array}{ll}\text { Samenvatting } & 193\end{array}$

$\begin{array}{ll}\text { Acknowledgements } & 194\end{array}$ 


\section{Chapter 1}

\section{Introduction}

\subsection{GFP and its mutants}

Intrinsically fluorescent proteins (FP's) [1,2] represent a very important class of photoactive biological systems which have launched a revolution in cell biology, being compatible with non-invasive imaging in living cells and allowing to dynamically visualize cellular processes in vivo in combination with conventional microscopy. Wild-type Aequorea green fluorescent protein (GFP) is the progenitor of the large family of FP's, which now covers almost the entire visible spectrum both in emission and absorption through the mutagenesis and continuous discovery of a variety of GFP-like proteins in different sea organisms [1]. The wide range of absorption and emission frequencies is for instance exploited in the Brainbow technique [3], which, using mixtures of FP's of sufficiently different colors, creates up to 100 distinguishable labels and can discriminate individual neurons. Another very important class of mutants are photoswitchable proteins, which have allowed surpassing the diffraction limit in so-called super-resolution microscopy as a very sharp final image can be reconstructed from several snapshots in which only a fraction of the markers is turned on and located very precisely $[4,5]$.

Most FP's share the same $\beta$-barrel structure and their chromophores are similar or even identical to that of GFP. Given the rich photophysical and photochemical behavior of these proteins, it is well understood that even subtle variations in the protein environment surrounding the chromophore can induce significant changes in its spectral properties. One astonishing example of the delicate interaction between the chromophore and its surroundings is the difference between the green Dronpa and cyan fluorescent mTFP0.7. Even though these two proteins share the same chromophore and most residues in proximity of the chromophore are identical, mTFP0.7 has an absorption blue shifted by $50 \mathrm{~nm}$ (or $0.3 \mathrm{eV}$ ) compared to Dronpa (see Figure 1.1). By inspecting visually the crystal structures of these proteins, one can infer the direction of the change in absorption from the location of the hydrogen bonds: The excitation involves a partial charge transfer from the phenolic ring to the imidazolinone ring, so the additional bond on the phenolic side in mTFP0.7 will stabilize the ground state while the additional bond on the imidazolinone ring in Dronpa will 


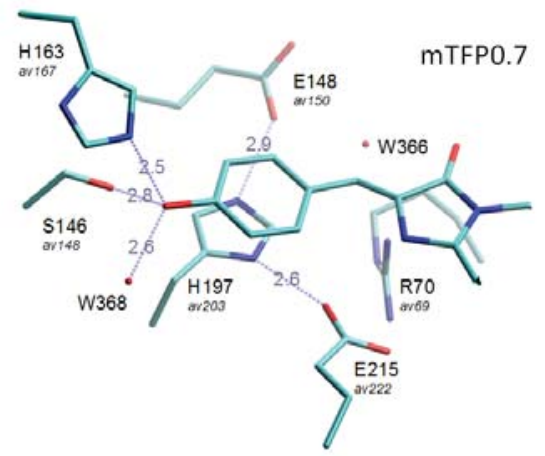

$453 \mathrm{~nm}(2.74 \mathrm{eV})$

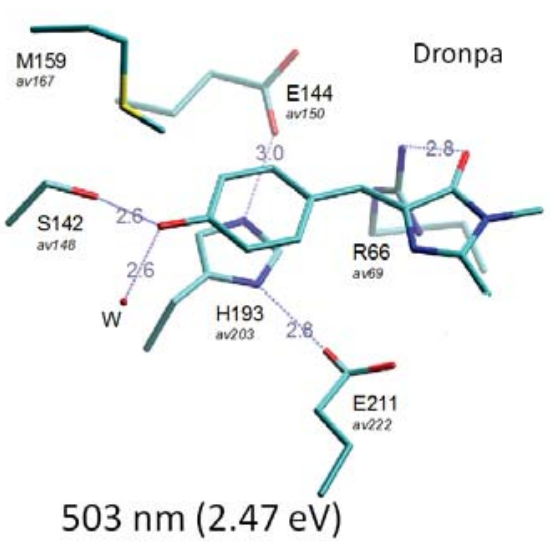

$503 \mathrm{~nm}(2.47 \mathrm{eV})$

Figure 1.1: Difference between mTFP0.7 and Dronpa structurally and in terms of absorption maxima. (Courtesy of R. Nifosì). The numbers with "av" refer to the position of the respective residues in wild-type (or aequorea victoria) GFP.

stabilize the excited state, therefore blue-shifting the absorption of mTFP0.7 with respect to Dronpa. However, the extent of the shift surpasses what one might expect from such little changes in non-covalent interactions. Since subtle variations in the protein environment can have large consequences on the absorption (and emission) properties of FP's, an indispensable feature of any computational model aimed at a quantitative description of these systems is the accurate treatment of the coupling between the chromophore and the protein.

Today, the design of new and more efficient FP's is an ongoing field of research $[1,2]$ as more advantageous mutants could further improve imaging techniques microscopy. For example, generating infrared- or red-fluorescent proteins (iRFP's or RFP's) is a desirable goal since light with longer wavelength is less phototoxic and is also less absorbed by the tissue, therefore being more suited for in vivo imaging [6-8]. For several years, the most red-shifted GFP mutant remained yellow fluorescent protein (YFP), [1] and red-emitting proteins were eventually generated from Anthozoa and not Aequorea (today, there are also GFP mutants that have red emission [9]). However, conventional RFP's often suffer from issues such as low brightness [7] or low photostability, and better mutants are necessary to bridge these shortcomings. In general, the design of novel FP's relies on phenomenological arguments and very approximate computational approaches are only used, for example, to screen for the stability of the $\beta$-barrel structure [10]. A recent review [11] notes, "For now, (semi-) random mutagenesis followed by high-throughput screening continues to be the technique of choice." A reliable computational strategy to excited-state modeling of these systems is in fact still elusive and this thesis takes a step into the direction of an ab initio electronic-structure description of the excitation process inside FP's, in particular, modeling absorption. We focus on absorption since predicting excitation energies is the first task in a computational study aimed at capturing any phenomenon in a FP, and if a model fails to accurately reproduce absorption (and shifts in absorption), other results it yields will also be dubious. 
The first test of the performance of a theoretical method is reproducing already known data. On that note, several recent studies [12-14] have attempted the computational characterization of these photoactive systems but reproduced trends in absorption between the various FP's with only limited degree of success. In this thesis, we will focus on wild-type GFP for several reasons. It has been extensively characterized experimentally as regards structure and spectral properties, making validation of the tested models easier. GFP is also a rare example of a fluorescent protein which has two significantly populated protonation forms at physiological $\mathrm{pH}$ and room temperature, which allows an additional route to comparison to experiments (i.e., the distance between the absorption maxima of the two forms) without changing the species. Finally, given that a large number of FP's are close mutants of GFP, the conclusions reached about GFP will likely be transferable to other members of the FP family.

\subsubsection{Structure and photocycle of GFP}

The structure in GFP was first identified via X-ray crystallography in 1996 by Ormö et al. [15] The protein has a barrel structure created by $11 \beta$-sheets, in which the photoactive chromophore is firmly held. By fixing the chromophore, the protein plays a crucial role in enabling fluorescence, which is characterized by a significant quantum yield also at room temperature. This must be contrasted to the behavior in solution where the chromophore has instead a very low quantum yield at room temperature (less than 0.001 [16]) but does fluoresce at $77 \mathrm{~K}[17,18]$ when the low temperature inhibits its numerous available modes of relaxation [18-20].

The absorption spectrum of GFP has two clear peaks at $398 \mathrm{~nm}$ and $478 \mathrm{~nm}$ attributed to two different protonation forms of the chromophore, namely, the neutral A and the anionic B form, respectively. From the relative intensities of these two peaks, it is possible to infer that at room temperature, the ratio between the $\mathrm{A}$ and B form populations is about 6:1 [21], while the B form becomes energetically favorable at low temperature $(1.6 \mathrm{~K})$ with the peaks corresponding to the two forms moving closer [22]. At room temperature, upon excitation at $398 \mathrm{~nm}$, the neutral chromophore loses its proton to Glu222 through the proton-transfer wire consisting of a water molecule, Ser205, and Glu222, and transforms into an intermediate (I) form as illustrated in Figure 1.2. This intermediate is characterized by the same hydrogen-bond network as the A form and an anionic chromophore as the B form. From the I form, the protein can convert into the B form through a slow and rare process which requires conformational changes in the protein environment, chiefly a rotation of Thr203 [23]. More commonly, it converts back into the A form [21].

In the protein pocket, there are in total four candidate residues that may bond to the phenolic side of the chromophore: Tyr145, His148, Thr203, and the aforementioned water molecule. The only two residues in the vicinity of the chromophore that may be charged are Arg96, which is always cationic and Glu222, which may be either protonated (neutral) or deprotonated (anionic) in the B and the A form, respectively $[23,24]$. We illustrate the location of the relevant residues in the A, I, and B forms along with the photocycle in Figure 1.2. 

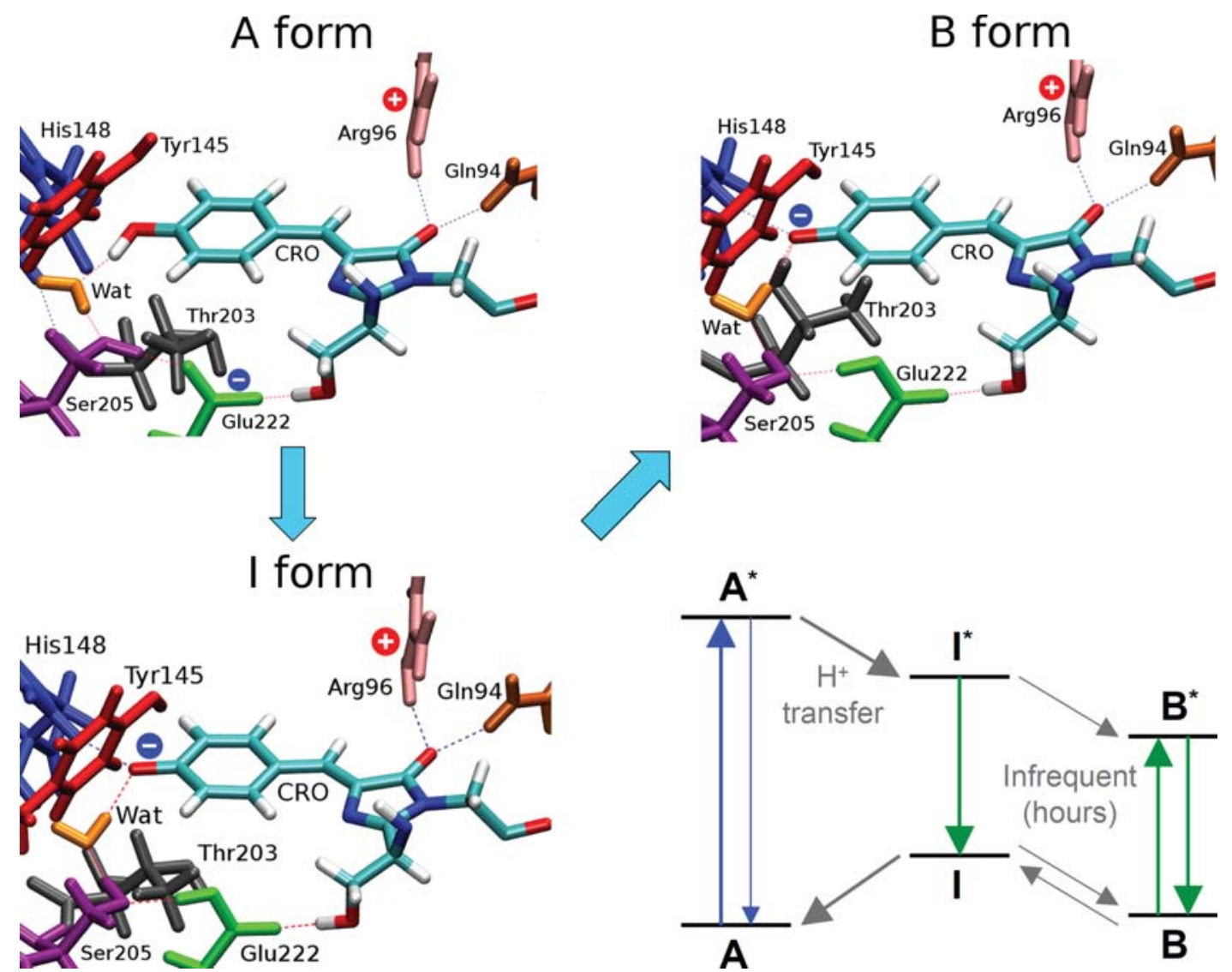

Figure 1.2: The transformation between the A and B forms of GFP through the intermediate I form. The conversion between the A and I forms is due to a proton transfer from the chromophore to Glu222, resulting in an anionic chromophore and a neutral Glu, while the conversion between the I and B forms is due to a change in conformation of Thr203. Bottom right: The photocycle between A, B, and I forms.

Given the size of the protein, about 2000 heavy atoms, a full quantum mechanical description of the system is clearly impossible and a multiscale approach is required: An expensive electronic-structure method must be employed for the chromophore and the protein environment must be treated at a lower level of theory. However, there is a wide range of choices available for how to perform the electronic-structure calculation and describe the coupling with the environment. Important criteria for choosing a combination of methods are computational cost, accuracy, and, critically, the number of (semi-)empirical parameters which should be as small as possible to allow us to work in a predictive framework as opposed to fit the particular experiments at hand. This is a crucial consideration since, ideally, a model developed for one protein should be readily and successfully applicable to any similar biosystem. The next subsection will explore the currently available multiscale methods and how they have been applied so far to FP's. 


\subsection{Complex environments and multiscale meth- ods: Point charges and beyond}

Based on the previous discussion, we can already dismiss some common multiscale methods. For example, we know that the protein environment is highly anisotropic and treating it as a continuum solvent will not be appropriate since describing each atom in the environment separately is an important ingredient. Furthermore, based on the relatively broad absorption spectrum and the change in the shape and position of the peaks at low temperatures, we can establish that thermal effects are also important for an accurate treatment of the system.

To reduce the computational cost for the molecular dynamics calculations at room temperature, we need the simplest available approximation for the atomistic environment, namely, quantum mechanics in molecular mechanics (QM/MM) [25, 26]. In this case, each atom in the environment is modeled as a single point charge and the interaction with the chromophore will be purely electrostatic (along with the relatively weak van der Waals forces). In general, QM/MM dynamics can be expected to yield reasonable molecular configurations since the force fields have been designed for this purpose. Nevertheless, once we choose a certain set of snapshots and wish to compute excitation energies on them, we may reasonably ask whether this point-charge description is sufficiently sophisticated to capture the subtle ways the environment can influence the excitation energies. Indeed, the bulk of the literature that employs this scheme seems to indicate that this level of treatment falls short of experimental agreement [13,27-33] and errors as large as $0.5 \mathrm{eV}$ in absorption have been recently reported for wild-type GFP [30]. What is the main aspect missing from the point-charge description?

To understand the reason why nonpolarizable classical descriptions fail, let us consider for instance the interaction between the positive counterion, Arg96, with the chromophore (see Figure 1.3). In the standard force field parameters, there is a positive charge of $0.75 e$ concentrated on the final seven atoms of guanidinium group: The amine groups are highly polar and attached to the significantly charged carbon atom. However, since the two nitrogen atoms and four hydrogen atoms are equivalent in an isolated arginine residue, their point charges will be fixed as equal in the force field and the interaction with the partially negative oxygen in the chromophore will not be taken into account. The frozen point charges will therefore miss (or at best, include very superficially) the various electronic interactions with the hydrogen-bond network and additional degrees of freedom are necessary to make the environment truly interact with the chromophore.

The most common and popular way to enhance the description of the ground state is through polarizable dipoles [34-37]. In this case, alongside the point charges mentioned before, each atom also possesses a polarizability and an induced dipole moment relating to it. Going back to our example, the different hydrogen bonds and local environments of the sites of the arginine residue will cause subtle variations in the dipole moments. However, including these additional degrees of freedom raise an additional issue: How to obtain the polarizabilities in the environment? In prac- 


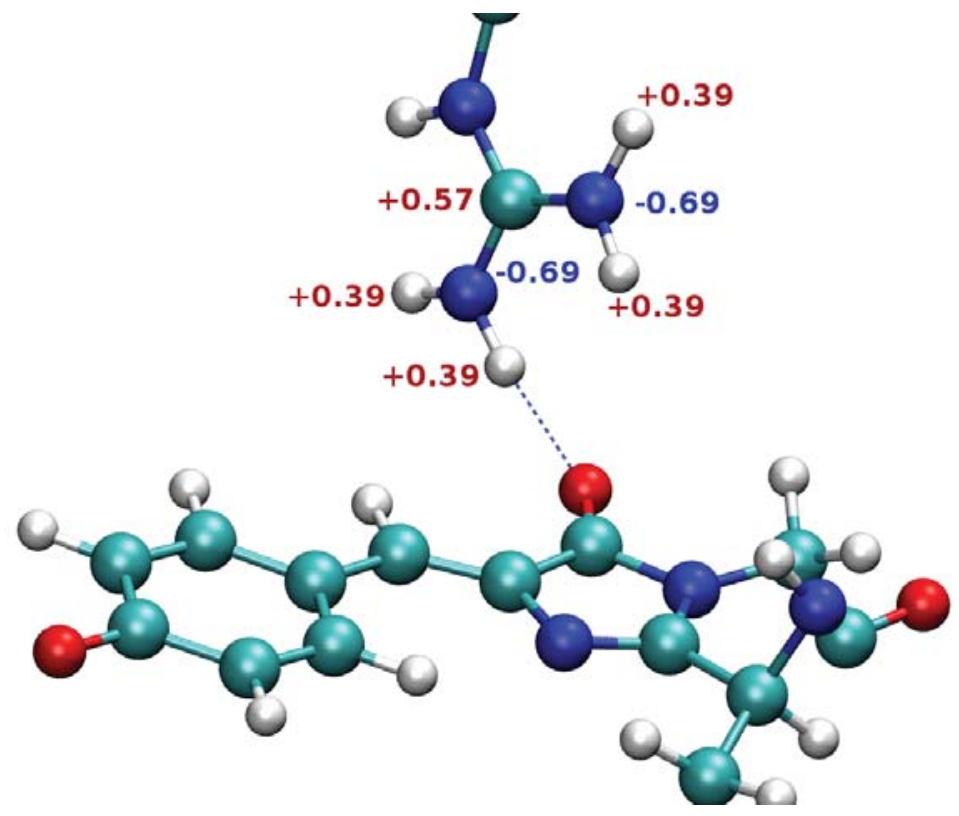

Figure 1.3: The point charges of the closest atoms to the chromophore in the counterion Arg96.

tice, additional experimental fits [38,39] are often performed, thereby even further distancing us from our initial goal of reducing the number of needed empirical parameters to the minimum. It is also possible to compute polarizabilities through ab initio calculations, for example, the popular LoProp method [40], but a certain amount of uncertainty relating to the chosen computational details (geometry, basis set, and quantum method) will still remain.

A more advanced way of introducing coupling between environment and chromophore is frozen-density embedding [41-43]. In this case, the environment is described as a continuous density computed at the quantum level through density functional theory (DFT). In principle, this scheme should significantly improve a classical description in several ways: One eliminates the majority of the empiricism (even the one related to the point charges) of the scheme, retaining only the (relatively few) parameters that had been used to develop the functionals needed in the scheme. Furthermore, one includes coupling beyond electrostatics such as Pauli repulsion. A drawback of DFT embedding is that computations become more expensive and one can include only part of the environment as a DFT density. However, in practice, the bottleneck of a calculation lies in the highly correlated method used to compute the excitation of the active site and reasonable sizes of quantum environments are feasible (see, for example, Figure 1.4). In fact, the treatment of an entire protein at the level of DFT embedding is possible if we split the system into several fragments $[44,45]$. In summary, as ground-state description, we would therefore expect DFT embedding to be a superior choice to polarizable dipoles.

On may however also ask if it is necessary to improve the description of the excited state as well. The excitation of the chromophore involves a certain degree of charge redistribution: In wild-type GFP, the phenolic ring loses some charge to the 


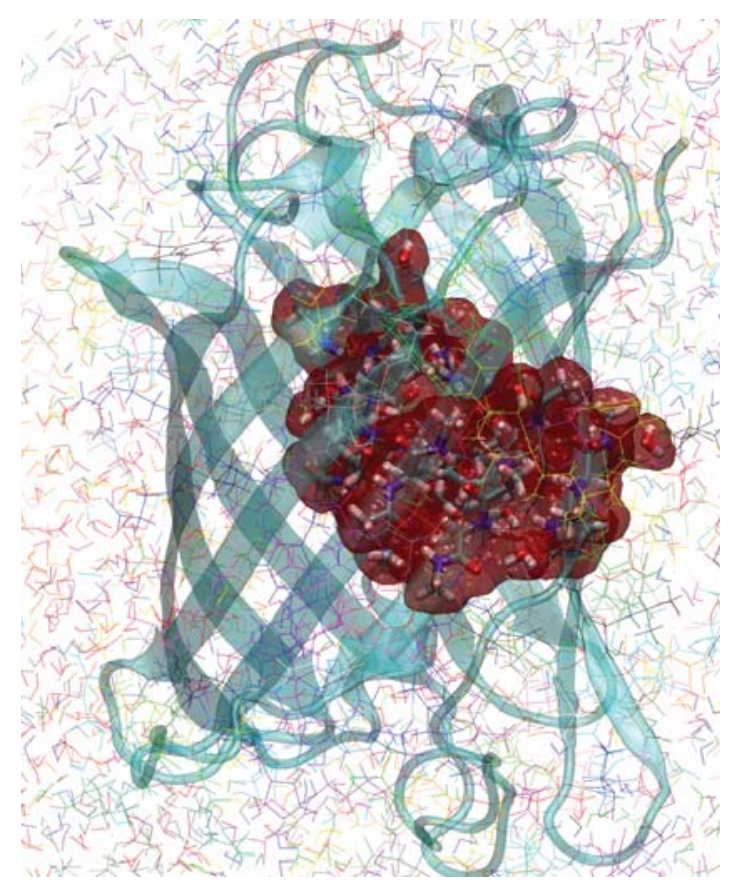

Figure 1.4: The size and position in the GFP barrel of a 344-atom cluster (in red) which is at the limit of what can be treated at a quantum level.

imidazolinone ring. At first sight, one might assume that this reorganization of charge has relatively little effect on the net electrostatic interaction: The chromophore contains more than 100 electrons and the mainly highest-occupied to lowest-unoccupied molecular orbital (HOMO $\rightarrow$ LUMO) excitation (see Figure 1.5) only involves the internal transfer of approximately one of these electrons. However, we must remember that most of the electric field created by the electrons will be canceled by the nuclei and it is more useful to think of the charge distribution in terms of a net dipole created by the whole chromophore. It turns out that, in wild-type GFP, this dipole changes significantly due to the excitation: For example, in the case of the A form, the excitation induces a change in the total dipole of the chromophore by a vector about $50 \%$ its size (see Figure 1.6). Based on this observation, we can expect this charge reorganization to polarize the environment in a different way than in the ground state. The change in the environment in response to the excitation of the embedded species will in turn affect the the excitation energy, introducing so-called "differential polarization" effects (and inducing a red shift in the excitation energy since the excited state is allowed more degrees of freedom). In the case of polarizable dipoles, including differential polarization in the model seems to be a straightforward task. The dipoles can readjust to any change in the density of the active subsystem to reach equilibrium in the excited state as well. We will return to this point in due course since the implementation of this effect actually strongly depends on the class of quantum method involved.

However, given that our "preferred" ground-state description is in principle DFT embedding, it is reasonable to treat differential polarization at the same level of the- 


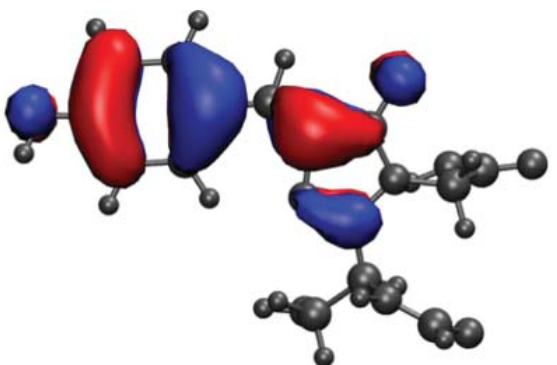

HOMO

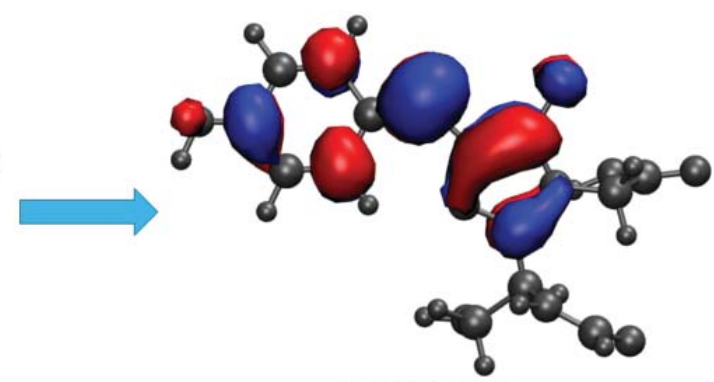

LUMO

Figure 1.5: The highest occupied and lowest unoccupied molecular orbitals (HOMO and LUMO) of the neutral chromophore of GFP.

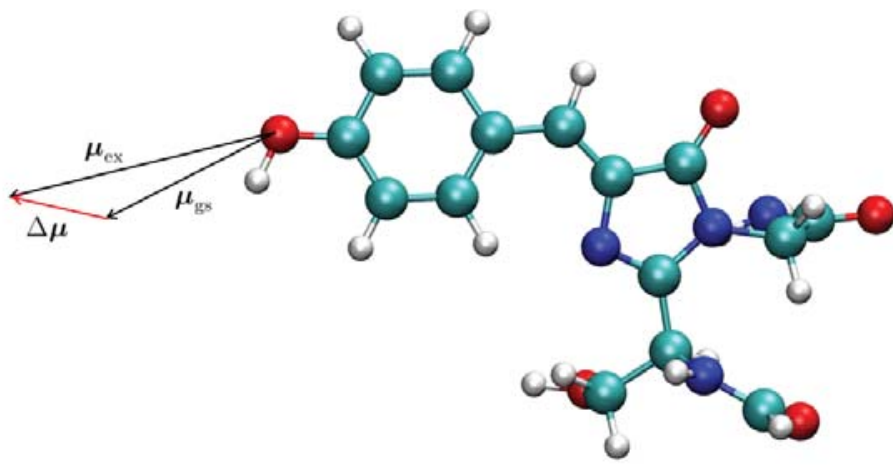

Figure 1.6: A form: The electrostatic dipole created by the chromophore in the ground state $\left(\boldsymbol{\mu}_{\mathrm{gs}}\right)$, excited state $\left(\boldsymbol{\mu}_{\mathrm{ex}}\right)$, and the difference between the two $(\Delta \boldsymbol{\mu})$. The relative magnitudes and directions of the dipoles are accurate but their origin and absolute size are chosen arbitrarily for pictorial reasons. The dipoles are computed with a wave function method (CASSCF) on the chromophore embedded in point charges (QM/MM).

ory, and have two different environmental densities and embedding potentials for the two states of interest. However, at the time of beginning our study, the formalism of differential polarization within DFT embedding had not been established yet. Therefore, we have taken the route of method development first, derived the necessary equations in Chapter 3, and tested the scheme further in Chapter 4, before applying it to our biological system of choice, GFP, in Chapter 5. For the sake of deeper understanding and to also facilitate a comparison with other studies in the literature, we also employ the simpler polarizable dipoles, which have in fact greatly helped us to gain a more complete picture of the origin of the chromophore-protein coupling.

In addition to the choice of the approach to describe the environment, we also need to choose a quantum method to treat the chromophore, a choice which can have very large effects on the final results. Significant spreads between excitation 
energies of different putatively accurate excited-state quantum methods are not uncommon, even in the case of calculations on small molecules done in vacuum [46] (see also Chapter 7). In this thesis, we will not take a definitive stance on the optimal choice of quantum methods and will instead employ more than one method whenever possible to eliminate the danger of a coincidental (or deliberate!) experimental agreement due to only a cancellation of errors. As an illustration of the effects of choosing a quantum method, we can mention two recent studies which have reported experimental agreement for GFP without accounting for any response from the environment, using however unconventional quantum methods [47,48]. In both cases, the quantum methods employed are documented to produce artificial red shifts, and thereby provide a counterbalance for the coarseness created by the lack of environmental response. Studies that directly determined the effect of differential polarization, however, $[14,33,49-52]$ have invariably arrived at the conclusion that it significantly affects the excitation. Choosing the wrong quantum method without careful analysis can in fact lead to qualitatively wrong conclusions: One may be satisfied by the experimental agreement and conclude that the environmental description is sufficient whereas in fact both the chromophore and the environment are inadequately modeled but causing opposite errors.

In the following section, we will give a short overview of how the chapters of this thesis fit together, moving from method development (in particular, differential polarization within DFT embedding) to the application of our new scheme to GFP.

\subsection{This thesis}

We introduce in Chapter $\mathbf{3}$ our novel wave function in density functional theory (WF/DFT) embedding technique we have developed to account for the response of the environment to the electronic excitation of the embedded species and for possible back-polarization effects on the excitation. In our hybrid scheme, density-based embedding potentials are constructed which differ for the ground and the excited states of interest, and are then employed in highly correlated calculations of the excited properties of the solute. We test our state-specific approach on the excited states of several small molecules solvated in both polar and nonpolar solvents, where the excitation is characterized by internal charge transfer and can strongly couple to the environment. The size of the solute-solvent clusters is such that calculations for the complete systems are feasible and, therefore, a direct comparison between our embedding approach and the supermolecular reference possible. For the four small molecules we used to test our model, differential polarization always improves or leaves unchanged the frozen DFT embedding results. Although, in several cases, the difference between the excitation energies obtained with the two DFT embedding schemes are small, one does not know in advance whether the environment responds to the excitation of the embedded species and our novel approach serves therefore as a useful diagnostic tool to assess the importance of this effect.

While the results of Chapter 3 are generally encouraging, several questions remain unanswered: First, how dependent is our scheme on the particular wave func- 
tion method used to describe the active site? Second, when the embedding method does not yield an improvement (or even worsens the results) compared to the use of a frozen, non-responsive environment, what is the origin of the error? Third, which is the best strategy to polarize the environment around the photo-excited active site? We answer these questions in Chapter $\mathbf{4}$ by considering several different wave function methods, namely, coupled cluster and quantum Monte Carlo methods in addition to the multi-determinant perturbation approach used in the previous chapter. We compare the response of these methods to a solvent described with DFT embedding and as a collection of explicit quantum water molecules. Our analysis suggests that problems in our responsive scheme can be often cured by polarizing the environment with respect to accurate excited-state solute densities. Remaining discrepancies with the reference are primarily due to the use of an approximate kinetic-energy functional in the construction of the embedding potential. This issue must be the focus of further research and will be briefly touched on in Chapter 6 .

Having introduced and tested our scheme involving differential polarization effects in a DFT environment, we are ready to employ it on GFP. In Chapter 5, we investigate the ability of our method and a variety of state-of-the-art multiscale schemes in predicting the absorption properties of GFP to identify the key theoretical ingredients for an accurate description of the chromophore-protein coupling in this prototypical photosensitive biosystem. On the classical side, we employ the simplest, most widely used approach of static point charges to which we then add polarizable dipoles and, on the quantum side, our DFT-based hybrid method. We test these embedding schemes against calculations on a large active region comprising the chromophore and a first shell of surrounding amino acids. We find that, in contrast to what commonly portrayed in the literature, a model where the protein is static or even polarized to the ground state of the chromophore (either quantum mechanically or through classical dipoles) is inadequate, indicating the need to couple the photexcited chromophore to its surroundings. Surprisingly, our quantum embedding scheme which should account for the environmental response to the excitation fails to improve on this description. Similarly, the use of dipoles polarized to the excited state of the chromophore produces negligible results. The best performance is in fact lent by classical polarizable dipoles but only when these are treated in a linear-response framework. While, in vacuum, excitations computed by linearresponse quantum methods coincide with those obtained through the direct solution of the time-independent Schrödinger in the limit of exact eigenfunctions [53], these two formulations yield different results when combined with a classical environment which depends on the quantum state through the induced dipoles. While the selfconsistent computation of eigenstates and the dipoles captures the main electrostatic interaction between the chromophore and the protein, linear-response calculations will miss this electrostatic term but happen to capture the instantaneous coupling between the environmental dipoles and the transition dipole moment (TDM) of the excitation itself $[54,55]$, which is fundamentally a quantum effect. Given this quantum (often referred to as "dispersion-like") coupling between the excitation and the environmental polarizabilities, our conclusion is that ultimately, only calculations on large quantum regions can reliably recover the subtle interaction between the photo- 
excited chromophore and its environment in GFP.

Given that the quantum-in-quantum DFT-embedding results in Chapter 5 fell short of our expectations, we test in Chapter $\mathbf{6}$ a novel embedding method where the role of the kinetic-energy functional is supplanted by a direct reconstruction of the embedding potentials. While this route is significantly more costly than using approximate functionals, it eliminates the main source of errors in WF/DFT embedding calculations. Importantly, this work is the first step towards the development of a reconstruction scheme where the target density of the embedded site is in fact the one of an excited species. Finally, the availability of accurate embedding potentials is essential for the benchmarking of new approximations of the kinetic-energy functional. We give a first numerical demonstration of the method, proving that we can recover the embedding potential and the target density in a numerically stable procedure via freeze\&thaw cycles without the need of an (in general, costly) supermolecular calculation.

Finally, Chapter 7 deviates from the main topic of electronic excitations in complex environments, being a benchmark study of the excited states of small polyenes, namely, ethene and butadiene, in the gas phase. The importance of the work stems from a combination of elements, namely, the difficulty encountered by most theoretical methods in describing the low-lying excited states of polyenes and the use of a novel quantum Monte Carlo approach to reach the full configuration interaction limit, a task previously thought to be impossible even for these small molecules. With this tool, we provide a robust estimate of the lowest vertical excitation energies of these polyenes, which are found to be significantly higher than the corresponding experimental absorption maximum. Our results represent therefore a reliable, important reference for other methods, especially given the difficulty of benchmarking theory on more complex systems as the ones described in the previous chapters. 


\section{Bibliography}

[1] R. N. Day and M. W. Davidson, Chem. Soc. Rev. 38, 2887 (2009).

[2] R. H. Newman, M. D. Fosbrink, and J. Zhang, Chemical Reviews 111, 3614 (2011).

[3] J. Livet, T. A. Weisman, H. Kang, R. W. Draft, J. Lu, R. A. Bennis, J. R. Sanes, and J. W. Lichtman, Nature 450, 56 (2007).

[4] S. W. Hell, Science 316, 1153 (2007).

[5] G. H. Patterson, Seminars in Cell \& Developmental Biology 20, 886 (2009).

[6] N. C. Deliolanis, R. Kasmieh, T. Würdinger, B. A. Tannous, K. Shah, and V. Ntziachristos, J. Biomed. Opt. 13, 044008 (2008).

[7] M. W. Davidson and R. E. Campbell, Nature Methods 6, 713 (2009).

[8] D. M. Shcherbakova and V. V. Verkhusha, Nature Methods 10, 751 (2013).

[9] A. S. Mishin, F. V. Subach, I. V. Yampolsky, W. King, K. A. Lukyanov, and V. V. Verkhusha, Biochemistry 47, 4666 (2008).

[10] R. A. Chica, M. M. Moore, B. D. Allen, and S. L. Mayo, Proc. Natl. Acad. Sci. 107, 20257 (2010).

[11] P. Dedecker, F. C. De Schryver, and J. Hofkens, J. Am. Chem. Soc. 135, 2387 (2013).

[12] J.-Y. Hasegawa, K. Fujimoto, B. Swerts, T. Miyahara, and H. Nakatsuji, J. Comput. Chem. 28, 2443 (2007).

[13] P. Amat and R. Nifosi, J. Chem. Theory Comput. 9, 497 (2013).

[14] M. T. Beerepoot, A. H. Steindal, J. Kongsted, B. O. Brandsdal, L. Frediani, K. Ruud, and J. M. Olsen, Phys. Chem. Chem. Phys. 15, 4735 (2013).

[15] M. Ormo, A. B. Cubitt, K. Kallio, L. A. Gross, R. Y. Tsien, and S. J. Remington, Science 273, 1392 (1996).

[16] W. W. Ward, C. W. Cody, R. C. Hart, and M. J. Cormier, Photochem. Photobiol. 31, 611 (1980). 
[17] H. Niwa, S. Inouye, T. Hirano, T. Matsuno, S. Kojima, M. Kubota, M. Ohashi, and F. I. Tsuji, Proc. Natl. Acad. Sci. 93, 13617 (1996).

[18] N. M. Webber, K. L. Litvinenko, and S. R. Meech, The Journal of Physical Chemistry B 105, 8036 (2001).

[19] K. L. Litvinenko, N. M. Webber, and S. R. Meech, Chem. Phys. Lett. 346, 47 (2001).

[20] D. Mandal, T. Tahara, N. M. Webber, and S. R. Meech, Chem. Phys. Lett. 358, 495 (2002).

[21] M. Chattoraj, B. A. King, G. U. Bublitz, and S. G. Boxer, Proc. Natl. Acad. Sci. 93, 8362 (1996).

[22] T. M. H. Creemers, A. J. Lock, V. Subramaniam, T. M. Jovin, and S. Völker, Nat. Struct. Biol. 6, 557 (1999).

[23] K. Brejc, T. K. Sixma, P. A. Kitts, S. R. Kain, R. Y. Tsien, M. Ormö, and S. J. Remington, Proc. Natl. Acad. Sci. 94, 2306 (1997).

[24] D. Stoner-Ma, A. A. Jaye, P. Matousek, M. Towrie, S. R. Meech, and P. J. Tonge, J. Am. Chem. Soc. 127, 2864 (2005).

[25] A. Warshel and M. Levitt, J. Mol. Biol. 103, 227 (1976).

[26] H. M. Senn and W. Thiel, Ang. Chem. Int. Ed. 48, 1198 (2009).

[27] M. Wanko, M. Hoffmann, T. Frauenheim, and M. Elstner, J. Phys. Chem. B 112, 11462 (2008).

[28] R. Send, V. R. I. Kaila, and D. Sundholm, J. Chem. Phys. 134, 214114 (2011).

[29] C. M. Isborn, A. W. Götz, M. A. Clark, R. C. Walker, and T. J. Martinez, J. Chem. Theory Comput. 8, 5092 (2012).

[30] C. Filippi, F. Buda, L. Guidoni, and A. Sinicropi, J. Chem. Theory Comput. 8, $112(2012)$

[31] V. R. I. Kaila, R. Send, and D. Sundholm, Phys. Chem. Chem. Phys. 15, 4491 (2013).

[32] O. Valsson, P. Campomanes, I. Tavernelli, U. Rothlisberger, and C. Filippi, J. Chem. Theory Comput. 9, 2441 (2013).

[33] T. Schwabe, M. T. Beerepoot, J. M. Olsen, and J. Kongsted, Phys. Chem. Chem. Phys. 17, 2582 (2015).

[34] M. A. Thomspon, J. Phys. Chem. 100, 14492 (1996). 
[35] C. Curutchet, A. Muñoz-Losa, S. Monti, J. Kongsted, G. D. Scholes, and B. Mennucci, J. Chem. Theory Comput. 5, 1838 (2009).

[36] J. M. Olsen, K. Aidas, and J. Kongsted, J. Chem. Theory Comput. 6, 3721 (2010).

[37] L. V. Slipchenko, J. Phys. Chem. A 114, 8824 (2010).

[38] J. Wang, P. Cieplak, J. Li, T. Hou, L. Ray, and D. Yong, J. Chem. Phys. B 8, 3091 (2011).

[39] J. Wang, P. Cieplak, J. Li, J. Wang, Q. Cai, M. Hsieh, H. Lei, R. Luo, and Y. Duan, J. Chem. Phys. B 8, 3100 (2011).

[40] L. Gagliardi, R. Lindh, and G. Karlström, The Journal of Chemical Physics 121, 4494 (2004).

[41] T. A. Wesolowski and A. Warshel, J. Phys. Chem. 97, 8050 (1993).

[42] A. S. P. Gomes, C. R. Jacob, and L. Visscher, Phys. Chem. Chem. Phys. 10, 5353 (2008).

[43] A. S. P. Gomes and C. R. Jacob, Annu. Rep. Prog. Chem., Sect. C 108, 222 (2012).

[44] O. Valsson, C. König, J. Neugebauer, and C. Filippi, private communication.

[45] C. König and J. Neugebauer, J. Chem. Theory Comput. 9, (2013).

[46] R. Send, O. Valsson, and C. Filippi, J. Chem. Theory Comput. 7, 444 (2011).

[47] B. L. Grigorenko, A. V. Nemukhin, I. Polyakov, D. I. Morozov, and A. I. Krylov, J. Am. Chem. Soc. 135, 11541 (2013).

[48] Q. Zhang, X. Chen, G. Cui, W.-H. Fang, and W. Thiel, Angewandte Chemie International Edition 53, 8649 (2014).

[49] A. H. Steindal, J. M. Olsen, K. Ruud, L. Frediani, and J. Kongsted, Phys. Chem. Chem. Phys. 14, 5440 (2012).

[50] A. Petrone, P. Caruso, S. Tenuta, and N. Rega, Phys. Chem. Chem. Phys. 15, 20536 (2013).

[51] M. T. Beerepoot, A. H. Steindal, K. Ruud, J. M. Olsen, and J. Kongsted, Comp. Theor. Chem. 1040-1041, 304 (2014).

[52] A. Pikulska, A. H. Steindal, M. T. P. Beerepoot, and M. Pecul, The Journal of Physical Chemistry B 119, 3377 (2015), pMID: 25646666.

[53] J. M. Olsen and P. Jorgensen, in Modern Electronic Structure Theory, Part II (World Scientific: Singapore, 1995). 
[54] R. Cammi, S. Corni, B. Mennucci, and J. Tomasi, J. Chem. Phys. 122, 104513 (2005).

[55] S. Corni, R. Cammi, B. Mennucci, and J. Tomasi, J Chem Phys 123, 134512 (2005). 


\section{Chapter 2}

\section{Theoretical Methods}

\subsection{Introduction}

We will employ several different electronic structure methods to study the excited states of systems having a wide range of sizes. Large systems will have to be treated at a computationally cheaper and more approximate level, while small systems can be handled at a higher computational cost and level of accuracy. We will invariably treat the excited states of our systems quantum mechanically, while the environment will be described more coarsely, possibly even classically. The main issues explored in this thesis relate to defining, and developing, the best computational strategy to combine accuracy and efficiency in a multiscale calculation of excited states. To avoid the pitfall of accepting a particular scheme based on a possibly coincidental agreement with experiments, we will employ a variety of quantum chemistry methods for the photoactive site in combination with several lower-level descriptions of the environment. Whenever feasible, we will compare multiscale results with supermolecular reference calculations.

In this chapter, we briefly describe the theoretical methods employed in this thesis. In particular, we will summarize the formalism of multi-configurational selfconsistent field (MCSCF) as well as its perturbative extensions, the quantum Monte Carlo (QMC) method, density functional theory (DFT), and time-dependent DFT (TDDFT). Likewise, we will explain how to couple these quantum methods with a lower-level description of the environment via a quantum mechanics-in-molecular mechanics (QM/MM) scheme and its polarizable variant (QM/MMpol), and conclude with a treatment of wavefunction-in-DFT embedding (WF/DFT).

\subsection{Quantum Mechanical Calculations}

We work here in the Born-Oppenheimer approximation [1,2], and neglect relativistic effects so that our non-relativistic system of $N$ interacting electrons is described by 
the Hamiltonian:

$$
\mathcal{H}=-\frac{1}{2} \sum_{i=1}^{N} \nabla_{i}^{2}+\sum_{i=1}^{N} v_{\text {ext }}\left(\mathbf{r}_{\mathbf{i}}\right)+\sum_{i<j}^{N} \frac{1}{\left|\mathbf{r}_{i}-\mathbf{r}_{j}\right|}
$$

where we used atomic units $\left(\hbar=m=e=1 / 4 \pi \epsilon_{0}=1\right)$. The external potential is either the bare electron-ion Coulomb potential $-Z / r$ where $Z$ is the charge of the ion, or a pseudopotential describing the ion plus the core electrons which have been eliminated from the calculation. We denote with $\mathrm{x}=(\mathbf{r}, \sigma)$ the 3 spatial and 1 spin coordinates of one electron where $\sigma= \pm 1$.

\subsubsection{Traditional Quantum Chemistry Methods}

In the Hartree-Fock (HF) method [1,2], one describes an interacting system of electrons with the optimal non-interacting wave function, namely, a Slater determinant of single-particle spin-orbitals $\left\{\Phi_{i}\right\}$ as

$$
\Psi_{\mathrm{HF}}\left(\mathbf{x}_{1}, \ldots, \mathbf{x}_{N}\right)=\frac{1}{\sqrt{N !}}\left|\begin{array}{cccc}
\Phi_{1}\left(\mathbf{x}_{1}\right) & \Phi_{1}\left(\mathbf{x}_{2}\right) & \ldots & \Phi_{1}\left(\mathbf{x}_{N}\right) \\
\Phi_{2}\left(\mathbf{x}_{1}\right) & \Phi_{2}\left(\mathbf{x}_{2}\right) & \ldots & \Phi_{2}\left(\mathbf{x}_{N}\right) \\
\vdots & \vdots & \vdots & \vdots \\
\Phi_{N}\left(\mathbf{x}_{1}\right) & \Phi_{N}\left(\mathbf{x}_{2}\right) & \cdots & \Phi_{N}\left(\mathbf{x}_{N}\right)
\end{array}\right|
$$

where the single-particle orbitals are determined by minimizing the expectation value of the interacting Hamiltonian on this wave function. The HF method is an important step for higher-order many-body methods, which usually contain linear combinations of determinants.

By expressing the spin-orbitals as the product of a spatial and a spin component, $\Phi_{i}(\mathbf{x})=\phi_{i}(\mathbf{r}) \chi_{s_{i}}(\sigma)$, and minimizing the energy subject to orthonormality constraints between the orbitals, one obtains the self-consistent HF equations for the spatial orbitals:

$$
\begin{aligned}
& {\left[-\frac{1}{2} \nabla^{2}+v_{\text {ext }}(\mathbf{r})+\sum_{j=1}^{N} \int \mathrm{d} \mathbf{r}^{\prime} \frac{\left|\phi_{j}\left(\mathbf{r}^{\prime}\right)\right|^{2}}{\left|\mathbf{r}-\mathbf{r}^{\prime}\right|}\right] \phi_{i}(\mathbf{r})} \\
& -\sum_{j=1}^{N} \delta_{s_{i}, s_{j}} \int \mathrm{d} \mathbf{r}^{\prime} \frac{\phi_{j}^{*}\left(\mathbf{r}^{\prime}\right) \phi_{i}\left(\mathbf{r}^{\prime}\right)}{\left|\mathbf{r}-\mathbf{r}^{\prime}\right|} \phi_{j}(\mathbf{r})=\epsilon_{i} \phi_{i}(\mathbf{r}) .
\end{aligned}
$$

The fully non-local HF exchange potential cancels the interaction of an electron with itself, that is, the self-interaction contribution from the the Hartree potential, and also implements the so-called Pauli exchange interaction so that electrons with the same spin tend to avoid each other.

The solution of a HF problem will entail starting from guess orbitals, determining the HF potential for these orbitals, and repeating the procedure until self-consistency (hence, the alternative name of the HF method being self-consistent field, SCF). 
For molecular systems, the orbitals are expanded as a linear combination of atomic orbitals (LCAO) centered on the nuclear positions:

$$
\phi_{i}(\mathbf{r})=\sum_{\mu}^{\text {nuclei }} \sum_{j} a_{j i}^{\mu} \eta_{j \mu}\left(\mathbf{r}-\mathbf{r}_{\mu}\right),
$$

where $\mathbf{r}_{\mu}$ denotes the position of a nucleus, and the minimization is performed with respect to the LCAO coefficients, $a_{j i}^{\mu}$. In most quantum chemistry codes, a Gaussian atomic basis is used:

$$
\eta(\mathbf{r})=x^{m} y^{n} z^{k} \exp \left(-\alpha r^{2}\right),
$$

as this choice allows all integrals to be computed analytically.

The difference between the exact energy $E$ and the HF energy is called the correlation energy, $E_{\text {corr }}=E-E_{\mathrm{HF}}$. The correlation energy is often considered as a sum of static correlation, which is linked to the insufficiency of a single-reference wave function, and dynamical correlation, which captures repulsions of the electrons beyond the mean-field approximation.

\section{Post Hartree-Fock Methods}

While the HF energy is missing all correlation effects, the full correlation energy can be recovered if one expresses the many-body wave function, $\Psi\left(\mathbf{x}_{1}, \ldots, \mathbf{x}_{N}\right)$, as an expansion in terms of all possible determinants of orthonormal single-particle orbitals, assuming our basis set is complete. This approach is called full configuration interaction (full CI or FCI). In practice, full CI is unfeasible for all but the smallest molecules and relatively modest basis sets, although a stochastic realization of this approach will be applied in Chapter 7 of this thesis for systems of up to 30 electrons and $10^{29}$ determinants. If we are using an atomic Gaussian basis set, the matrix elements of the Hamiltonian and the overlap of these $N$-electron basis functions can be expressed as integrals over one-electron orbitals and computed analytically.

The wavefunction in the configuration interaction (CI) approach is obtained by considering excitations out of the reference HF determinant to a set of virtual orbitals as

$$
\Psi_{\mathrm{CI}}=c_{0} D_{\mathrm{HF}}+\sum_{a b} c_{a \rightarrow b} D^{a \rightarrow b}+\sum_{a b c d} c_{a b \rightarrow c d} D^{a b \rightarrow c d}+\ldots
$$

where $D^{a \rightarrow b}$ denotes a single excitation where the occupied orbital $a$ in the HF determinant is substituted with the virtual orbital $b$. Similarly, $D^{a b \rightarrow c d}$ indicates a double excitation from $a$ and $b$ to the virtual orbitals $c$ and $d$. In practice, one often truncates this expansion to a low level: For example, CISD (configuration interaction singles and doubles) only includes single and double excitations.

If we include up to $N$-body excitations to all virtual orbitals, we obtain the full CI expansion, which must then be extrapolated to the infinite basis limit by considering a sequence of larger basis sets. If we denote with $C_{i}$ a spin- and space-adapted configuration state functions (CSF) (i.e. a fixed linear combination of determinants 
with proper spin and space symmetry), we can rewrite a CI expansion as

$$
\Psi_{\mathrm{CI}}=\sum_{i=1}^{K} c_{i} C_{i}
$$

and, by applying the variational principle, obtain the secular equations for the coefficients $c_{i}$ :

$$
\sum_{j=1}^{K}\left\langle C_{i}|\mathcal{H}| C_{j}\right\rangle c_{j}^{(k)}=E_{\mathrm{CI}}^{(k)} \sum_{j=1}^{K}\left\langle C_{i} \mid C_{j}\right\rangle c_{j}^{(k)},
$$

where $\left\langle C_{i} \mid C_{j}\right\rangle=\delta_{i j}$ as the orbitals are orthonormal.

For a CI expansion (and any linear expansion on a basis set), one obtains not only an approximation to the ground-state wave function but also to the excited states thanks to a generalized variational principle, known as the McDonald's theorem, which states that the approximate solutions with energies $E_{\mathrm{CI}}^{(0)} \leq E_{\mathrm{CI}}^{(1)} \leq \ldots \leq E_{\mathrm{CI}}^{(K)}$ satisfy

$$
E_{i} \leq E_{\mathrm{CI}}^{(i)}
$$

where $E_{i}$ are the exact energies of the eigenstates of the Hamiltonian $\mathcal{H}$. A CI wave function is however a slowly converging expansion and a large number of determinants must be included due to the lack of explicit dependence of the inter-electron coordinates, and the consequent poor description of the cusp occurring at the electronelectron coalescence points. Moreover, the number of determinants grows exponentially with the number of electrons $N$ and, while limiting the number of determinants to the most important excitations lowers the computational cost (for instance, CISD includes single and doubles and scales as $N^{6}$ ), it results in the loss of size consistency.

In the multi-configurational self-consistent field (MCSCF) approach, one minimizes the energy not only with respect to the linear coefficients $c_{i}$ but also the LCAO coefficients $a_{j i}$. The complete-active-space self-consistent field (CASSCF) approach is a particular type of MCSCF calculation, where $n$ electrons are distributed over a set of $m$ active orbitals, whose occupancy is allowed to vary. The resulting $\operatorname{CASSCF}(n, m)$ calculation is like a full CI calculation for $n$ electrons in $m$ orbitals, except that also the orbitals are now optimized to minimize the total energy. Outside the active space, there is generally an inactive space of orbitals that are doubly occupied in every determinant, but still influence the energy since they can change in the optimization, and there is the virtual space of unoccupied orbitals, which have no influence on the CASSCF energy but will be important in the perturbative expansions mentioned later.

When several states of the same symmetry are requested, it is customary to use the state averaged (SA) CASSCF approach to avoid root-flipping problems in the optimization. In a SA calculation, the weighted average of the energies of the states of interest is optimized:

$$
E_{\mathrm{SA}}=\sum_{I} w_{I} \frac{\left\langle\Psi_{I}|\mathcal{H}| \Psi_{I}\right\rangle}{\left\langle\Psi_{I} \mid \Psi_{I}\right\rangle}
$$


where $\sum_{I} w_{I}=1$ and the states are kept orthogonal. The optimization yields one common set of orbitals and the different states differ only in their CI coefficients, which ensure orthogonality. The most important step for a MCSCF/CASSCF calculation is the selection of the active space, which requires a fair amount of knowledge of the system under study and is rather time-consuming since a great number of trial calculations is often necessary. Furthermore, MCSCF/CASSCF calculations recover only a small part of the correlation energy as it only provides a description of nondynamical correlation.

One way to improve upon a MCSCF calculation is to use Rayleigh-Schrödinger perturbation theory, where the total Hamiltonian is partitioned into a zero-order Hamiltonian, $\mathcal{H}^{(0)}$, and a perturbing operator $\mathcal{V}$ :

$$
\mathcal{H}=\mathcal{H}^{(0)}+\mathcal{V}
$$

The definition of the perturbation theory is complete once we have decided on the zero-order wave function and Hamiltonian. If we take the zero-order wave function to be the HF wave function, we would obtain the Møller-Plesset perturbation theory (MP2). Taking a MCSCF wave function to be the zero-order wave function, we obtain the multi-reference perturbation theory (MRPT). Specifically, in the case of a CASSCF wave function, this has resulted in the complete active space perturbation theory (CASPT2) [3], which is one of the most widely used methods in excited-state quantum chemistry. However, for MRPT, there is no unique definition of the zero-order Hamiltonian and this has led to multiple formulations of CASPT2, which differ in the choice of the zero-order Hamiltonian yielding the same zero-order CASSCF wave function. For example, a more modern formulation of the zero-order one-body Hamiltonian employed in CASPT2 calculations involves an ionization potential-electronic affinity (IPEA) shift [4] of 0.25 a.u., which is a semiempirical parameter that compensates a discrepancy between orbital energies when exciting from and exciting into the active space. Excitation energies computed with the CASPT2 method without the IPEA shift are too low [5], which calls for particular care when making theoretical comparison to older works. A different formulation of MRPT also based on a CASSCF zero-order wave function is the so-called $n$-electron valence state perturbation theory (NEVPT2) [6], which is based on a more advanced zero-order Hamiltonian than CASPT2. In particular, the zero-order Hamiltonian in NEVPT2 explicitly includes two-electron terms for the active electrons, while the CASPT2 zero-order Hamiltonian only includes one-electron terms.

Finally, we also use coupled-cluster (CC) methods, which are similar to configuration interaction, but use an exponential excitation operator in the ansatz of the wave function:

$$
\left|\Psi_{\mathrm{CC}}\right\rangle=e^{\hat{T}}\left|\Psi_{\mathrm{HF}}\right\rangle,
$$

where $\hat{T}=\hat{T}^{1}+\hat{T}^{2}+\hat{T}^{3}+\ldots$ is the excitation operator grouping together single, double, triple, ... excitations. For example, the operator for the single-excitation class can be written as:

$$
\hat{T}^{1}=\sum_{i} \sum_{a} t_{a}^{i} \hat{a}_{a}^{\dagger} \hat{a}_{i}
$$


where $t_{a}^{i}$ are unknown excitation amplitudes.

In practice, as in the case of CI methods, this operator is truncated to define the CCS, CCSD [7], CCSDT [8,9], ... methods. The energy and excitation amplitudes are computed via the coupled-cluster equations which, for instance in CCSD, are given by:

$$
\begin{aligned}
\left\langle\Psi_{\mathrm{HF}}\left|e^{-\hat{T}} H e^{\hat{T}}\right| \Psi_{\mathrm{HF}}\right\rangle & =E_{\mathrm{CCSD}} \\
\left\langle\Psi_{i}^{a}\left|e^{-\hat{T}} H e^{\hat{T}}\right| \Psi_{\mathrm{HF}}\right\rangle & =0 \\
\left\langle\Psi_{i j}^{a b}\left|e^{-\hat{T}} H e^{\hat{T}}\right| \Psi_{\mathrm{HF}}\right\rangle & =0,
\end{aligned}
$$

where $\hat{T}=\hat{T}_{1}+\hat{T}_{2}$. There are several different routes which approximate coupledcluster equations at a reduced computational cost. For example, CCSD [7] can be approximated by CC2 [10-12], which scales as $N^{5}$ compared to $N^{6}$ in the case of CCSD.

A very important difference between $\mathrm{CC}$ and $\mathrm{CI}$ methods is that $\mathrm{CC}$ is size consistent: The sum of the energies of two systems computed separately is identical to that of the method applied to both subsystems taken together in the limit of infinite separation. This is true for any truncation of the coupled-cluster expansion. This property, which is due to the exponential ansatz of the CC wave function, comes however at a cost: While truncated CI methods are variational (they give an upper bound to the energy), the above energy estimator of a truncated $\mathrm{CC}$ wave function is not (it may recover more than $100 \%$ of the total correlation energy). This nonvariational character does not significantly affect the energies for small molecules close to the equilibrium condition. In particular, the coupled cluster theory with singles and doubles plus perturbative triples $[\operatorname{CCSD}(\mathrm{T})]$ has been very successful, in particular in small systems with low static correlation, and is often referred to as the "gold standard" of quantum chemistry.

Excited states can also be obtained in the coupled-cluster method through the so-called equation-of-motion coupled-cluster (EOM-CC) formalism by acting on the ground-state CC wave function with the excitation operators $\hat{R}_{i}$ (whose amplitudes are unknown):

$$
\left|\Psi_{i}\right\rangle=\hat{R}_{i}\left|\Psi_{0}\right\rangle=\hat{R}_{i} e^{\hat{T}}\left|\Psi_{\mathrm{HF}}\right\rangle
$$

Rearranging the coupled-cluster equations, we obtain that the excited-state wave functions are the right-eigenvectors of a non-Hermitian effective Hamiltonian $\bar{H}=$ $e^{-\hat{T}}\left(\hat{H}-E_{0}\right) e^{\hat{T}}$ :

$$
\bar{H} \hat{R}_{i}\left|\Psi_{\mathrm{HF}}\right\rangle=\omega_{i} \hat{R}_{i}\left|\Psi_{\mathrm{HF}}\right\rangle
$$

where $\omega_{i}=E_{i}-E_{0}$ gives the excitation energy at the coupled-cluster level. In practical calculations, the operators $\hat{R}$ are truncated to the same degree as the coupledcluster operator: For example, in EOM-CCSD, one would only include single and double excitations in $\hat{R}$. 


\subsubsection{Density Functional Theory}

Density functional theory (DFT) [13] is a very popular alternative to wave functionbased approaches due to its favorable scaling with system size. The DFT formalism is based on the electronic density, a 3-dimensional function, instead of the more impractical $3 \mathrm{~N}$-dimensional many-body wave function. While the Hohenberg-Kohn theorem [14] proves that the ground-state electron density uniquely determines the external potential and therefore the wave function together with all observables, the mapping functional is unknown. Therefore, compared to wave function-based methods where the basic equations are well known but often computationally intractable, DFT reduces the size of the problem by several orders of magnitude but introduces a fundamental unknown in the equations to solve. In practical DFT applications, one relies on the Kohn-Sham theorem [15], which maps the interacting system to a non-interacting one with the same ground-state density, and results in the simple single-particle equations:

$$
\left[-\frac{1}{2} \nabla^{2}+v_{\text {eff }}([\rho] ; \mathbf{r})\right] \phi_{i}=\epsilon_{i} \phi_{i}
$$

where the electronic density is constructed by summing over the $N$ lowest energy orbitals where $N$ is the number of electrons:

$$
\rho(\mathbf{r})=\sum_{i=1}^{N}\left|\phi_{i}(\mathbf{r})\right|^{2} .
$$

The effective Kohn-Sham potential is given by

$$
v_{\text {eff }}([\rho] ; \mathbf{r})=v_{\text {ext }}(\mathbf{r})+\int \frac{\rho\left(\mathbf{r}^{\prime}\right)}{\left|\mathbf{r}-\mathbf{r}^{\prime}\right|} \mathrm{d} \mathbf{r}^{\prime}+v_{\text {xc }}([\rho] ; \mathbf{r}),
$$

where $v_{\text {ext }}(\mathbf{r})$ is the external potential. The exchange-correlation potential $v_{\mathrm{xc}}([\rho] ; \mathbf{r})$ is the functional derivative of the exchange-correlation energy $E_{\mathrm{xc}}[\rho]$ that enters in the expression for the total energy:

$$
\begin{aligned}
E & =-\frac{1}{2} \sum_{i=1}^{N} \int \phi_{i} \nabla^{2} \phi_{i} \mathrm{~d} \mathbf{r}+\int \rho(\mathbf{r}) v_{\text {ext }}(\mathbf{r}) \mathrm{d} \mathbf{r} \\
& +\frac{1}{2} \iint \frac{\rho(\mathbf{r}) \rho\left(\mathbf{r}^{\prime}\right)}{\left|\mathbf{r}-\mathbf{r}^{\prime}\right|} \mathrm{d} \mathbf{r} \mathrm{d} \mathbf{r}^{\prime}+E_{\mathrm{xc}}[\rho] .
\end{aligned}
$$

The exchange-correlation functional is the aforementioned unknown component of DFT: Even though DFT is in principle exact, the exchange-correlation energy must be approximated.

\section{Approximate Exchange-Correlation Functionals}

Several approximate exchange-correlation functionals have been proposed in the literature [16], and recent years have in fact seen a proliferation of novel functionals, 
sometimes designed for the study of specific properties. In this thesis, we employ the local-density approximation (LDA), various generalized gradient approximation (GGA), hybrid, meta-hybrid, and long-range corrected (LC) functionals. The hierarchy of these functionals has been famously likened by John Perdew to the "Jacob's Ladder" between the "Hartree hell" and the "heaven of chemical accuracy" [17].

The LDA is the simplest functional:

$$
E_{\mathrm{xc}}^{\mathrm{LDA}}[\rho]=\int \mathrm{d} \mathbf{r} \epsilon_{\mathrm{xc}}^{\mathrm{hom}}(\rho(\mathbf{r})) \rho(\mathbf{r})
$$

where $\epsilon_{\mathrm{xc}}^{\mathrm{hom}}(\rho)$ is the exchange-correlation energy per electron of a uniform electron gas of density $\rho$. The generalized gradient approximation (GGA) introduces a dependence on the gradient and higher derivatives of the density, whose generic expression (here restricted to second-order derivatives) is given by

$$
E_{\mathrm{xc}}^{\mathrm{GGA}}[\rho]=\int \rho(\mathbf{r}) \epsilon_{\mathrm{xc}}^{\mathrm{GGA}}\left(\rho(\mathbf{r}),|\nabla \rho(\mathbf{r})|, \nabla^{2} \rho(\mathbf{r})\right) \mathrm{d} \mathbf{r} .
$$

Out of the class of GGA's, we will employ the Becke-Lee-Yang-Parr (BLYP) $[18,19]$ for some geometry optimizations in the gas phase and the Perdew-Burke-Ernzerhof (PBE) [20] functional in the ground-state ab initio molecular dynamics simulations. In our embedding calculations, we also use the Perdew-Wang exchange-correlation functional (PW91) [21].

Hybrid functionals introduce a dependence on the Kohn-Sham orbitals by mixing in the functional a portion of exact exchange from Hartree-Fock theory:

$$
E_{\mathrm{x}}^{\mathrm{HF}}[\rho]=-\frac{1}{2} \sum_{i=1}^{N} \sum_{j=1}^{N} \delta_{s_{i}, s_{j}} \iint \frac{\phi_{i}^{*}(\mathbf{r}) \phi_{j}^{*}\left(\mathbf{r}^{\prime}\right) \phi_{j}(\mathbf{r}) \phi_{i}\left(\mathbf{r}^{\prime}\right)}{\left|\mathbf{r}-\mathbf{r}^{\prime}\right|} \mathrm{d} \mathbf{r} \mathrm{d} \mathbf{r}^{\prime} .
$$

Mixing of exact exchange in the functional expression was introduced by Becke [22] based on the adiabatic connection formalism (which provides a path at constant density between the Kohn-Sham non-interacting system and the interacting one) and further rationalized in Ref. 23: At the limit of no Coulomb repulsion, the exchangecorrelation energy is identical to the Hartree-Fock exchange with Kohn-Sham orbitals, while at full Coulomb repulsion, semilocal functionals are more appropriate. The simplest approximation (assuming a linear switching between the two regimes) would then imply a 50\% exact exchange, a so-called "half-and-half" functional [22]. More sophisticated combinations have been developed, a particularly widely used one being the three parameter B3LYP functional $[24,25]$ which combines LDA and the BLYP GGA with $20 \%$ exact exchange. We employ the B3LYP functional for geometry optimization and in some cases for the construction of the embedding potentials.

Meta-GGA functionals introduce a dependence on the local non-interacting kinetic density:

$$
\tau=\frac{1}{2} \sum_{i}\left|\nabla \phi_{i}(\mathbf{r})\right|^{2} .
$$


Just like normal GGA's, meta-GGA functionals can and have been combined with exact exchange, resulting in the so-called meta-hybrid functionals. A popular family among these functionals is the M06 [26-29] set of four functionals that differ mainly in the amount of exact exchange included; M06-L (0\%), M06 (27\%), M06$2 \mathrm{X}(54 \%), \mathrm{M} 06-\mathrm{HF}(100 \%)$. We use the M06-HF functional for the construction of most embedding potentials since this functional ensures in general a better convergence for systems with separated charges (as one may encounter when performing a DFT calculation of a protein fragment containing close-by charged amino acids).

Finally, we consider the long-range corrected functionals (a good overview of them can be found in Ref. 30), where one splits the electron-electron interaction into a short- and a long-range component

$$
\frac{1}{r}=\frac{1-g(r)}{r}+\frac{g(r)}{r},
$$

where, for instance, $g(r)=\operatorname{erf}(\mu r)$. In the original proposal of Iikura, Tsuneda, Yanai, and Hirao [31], the short-range component is treated through a density functional approximation (DFA) while the long-range component is considered as exact exchange, resulting in

$$
E_{\mathrm{xc}}[\rho]=E_{\mathrm{x}}^{\mathrm{HF}, \mathrm{LR}}[\rho]+E_{\mathrm{x}}^{\mathrm{DFA}, \mathrm{SR}}[\rho]+E_{\mathrm{c}}^{\mathrm{DFA}}[\rho] .
$$

The usage of the error function allows the exact exchange part to be obtained analytically while, for the short-range part, a semi-local density functional approximation is used $[16,31]$. The parameter $\mu$ controls the range of separation and a value of $0.3-0.5$ a.u. is often found to be optimal.

As given above, the long-range corrected functionals include $0 \%$ exact exchange at short range and $100 \%$ exact exchange at long range. However, the formulation has been generalized to include a certain amount of exact exchange at both short and long ranges as proposed in the so-called Coulomb-attenuating method (CAM) [32]:

$$
\begin{aligned}
E_{\mathrm{xc}}[\rho] & =a E_{\mathrm{x}}^{\mathrm{HF}, \mathrm{SR}}[\rho]+b E_{\mathrm{x}}^{\mathrm{HF}, \mathrm{LR}}[\rho] \\
& +(1-a) E_{\mathrm{x}}^{\mathrm{DFA}, \mathrm{SR}}[\rho]+(1-b) E_{\mathrm{x}}^{\mathrm{DFA}, \mathrm{LR}}[\rho]+E_{\mathrm{c}}^{\mathrm{DFA}}[\rho] .
\end{aligned}
$$

In particular, the popular CAM-B3LYP functional [32] uses short- and long-range versions of the B88 functional and the same correlation functional used in B3LYP, and includes $19 \%$ exact exchange at short range $(a=0.19)$ and only $65 \%$ at long range ( $b=0.65)$ with $\mu=0.33$ a.u. It has been observed that the use of long-range corrected functionals can ameliorate the well-known shortcoming of other functional forms in describing charge-transfer excitations within time-dependent DFT.

In this thesis, we will employ the CAM-B3LYP functional [32] and the LC-BLYP functional (which uses a short-range version of the B88 functional for exchange, the LYP functional for correlation, $a=0, b=1$, and $\mu=0.47$ a.u.) in the study of the excited states of green fluorescent protein (GFP). We note in passing that alternative range-separated functionals also exist [33], in which the opposite separation is used with the short-range component containing more exact exchange than the long-range 
one. These were developed to simplify the calculation of the non-local exchange interaction in periodic systems.

Finally, an important actor in the DFT embedding methods describe below is the kinetic-energy density functional (KEDF) for orbital-free DFT (i.e., without the KS formalism). The simplest KEDF is the Thomas-Fermi functional [34-36], which is the LDA for the kinetic energy functional, namely, the kinetic energy of an uniform electron gas:

$$
T[\rho]=C_{F} \int[\rho(\mathbf{r})]^{5 / 3} \mathrm{~d} \mathbf{r} .
$$

We will usually employ the Perdew-Wang kinetic-energy functional (PW91k) [37], which is a re-parametrization of the PW91 [38] exchange-correlation functional with the correct Thomas-Fermi limit for homogeneous densities.

\section{Time-Dependent Density Functional Theory}

Time-dependent density-functional theory (TDDFT) [39] represents a rigorous formalism to compute response properties of molecules, but is most widely applied to determine excitation energies through the dynamic polarizability tensor. As in the case of ground-state DFT, while exact in principle, TDDFT also depends on the use of approximate exchange-correlation functionals alongside other approximations mentioned below.

The time-dependent equivalent of the Hohenberg-Kohn theorem is the RungeGross theorem [40]. It proves the one-to-one correspondence between the external time-dependent potential $v_{\text {ext }}(\mathbf{r}, \mathrm{t})$ and the time-dependent electronic density, $\rho(\mathbf{r}, \mathrm{t})$ and leads to the construction of a time-dependent Kohn-Sham scheme. The interacting problem is then exactly mapped to a system of non-interacting electrons in an effective external time-dependent potential:

$$
\left[-\frac{1}{2} \nabla^{2}+v_{\mathrm{eff}}([\rho] ; \mathbf{r}, t)\right] \phi_{i}(\mathbf{r}, t)=i \frac{\partial}{\partial t} \phi_{i}(\mathbf{r}, t)
$$

which yields the exact electronic density constructed from the Kohn-Sham orbitals as

$$
\rho(\mathbf{r}, t)=\sum_{i=1}^{N}\left|\phi_{i}(\mathbf{r}, t)\right|^{2} .
$$

The Kohn-Sham effective potential is given by:

$$
v_{\text {eff }}([\rho] ; \mathbf{r}, t)=v_{\text {ext }}(\mathbf{r}, t)+\int \frac{\rho\left(\mathbf{r}^{\prime}, t\right)}{\left|\mathbf{r}-\mathbf{r}^{\prime}\right|} \mathrm{d} \mathbf{r}^{\prime}+v_{\mathrm{xc}}([\rho] ; \mathbf{r}, t) .
$$

Here, it is important to note that the time-dependent exchange-correlation potential is not the same functional of the density as the ground-state exchange-correlation potential (Eq. 2.18). Instead, it is the functional derivative of the exchange-correlation component of the action functional $[40,41]$. 
Some approximations are needed as the time-dependent exchange-correlation potential is unknown. The simplest approximation is to assume that the exchangecorrelation potential is local in time, that is, reacts instantaneously and without memory to any temporal change of the density. This is known as the adiabatic approximation:

$$
v_{\mathrm{xc}}^{\mathrm{adiab}}([\rho] ; \mathbf{r}, t)=\left.v_{\mathrm{xc}}^{\mathrm{gs}}([\rho] ; \mathbf{r})\right|_{\rho=\rho(\mathbf{r}, t)},
$$

where $v_{\mathrm{xc}}^{\mathrm{gs}}$ is a particular chosen ground-state exchange-correlation potential. Here, $v_{\mathrm{xc}}^{\mathrm{gs}}$ is a property of the ground-state, so this approximation should work best for timedependent systems where the density is not too far from the ground-state density.

If we know how the system responds to a small time-dependent perturbation, then the excitation energies can easily be obtained from a TDDFT calculation. To achieve this, the key quantity is the linear density response function $\chi$ that gives the change in the density if the system is subjected to a small perturbation in the external potential:

$$
\delta \rho(\mathbf{r}, \omega)=\int \mathrm{d} \mathbf{r}^{\prime} \chi\left(\mathbf{r}, \mathbf{r}^{\prime}, \omega\right) \delta v_{\mathrm{ext}}\left(\mathbf{r}^{\prime}, \omega\right)
$$

Using the linear density response function, we can compute the dynamic polarizability and consequently obtain the photoabsorption cross section. We can rewrite this change in the density by using the time-dependent Kohn-Sham scheme (Eqs. 2.282.30 ), resulting in

$$
\delta \rho(\mathbf{r}, \omega)=\int \mathrm{d} \mathbf{r}^{\prime} \chi_{\mathrm{KS}}\left(\mathbf{r}, \mathbf{r}^{\prime}, \omega\right) \delta v_{\mathrm{eff}}\left(\mathbf{r}^{\prime}, \omega\right) .
$$

Here, $\chi_{\mathrm{KS}}$ is the density response function for the non-interacting Kohn-Sham electron system, which we can write in the terms of the unperturbed time-independent Kohn-Sham orbitals. We can obtain the linear change in the potential if we make use of the definition of the exchange-correlation potential (Eq. 2.29):

$$
\delta v_{\mathrm{eff}}(\mathbf{r}, \omega)=\delta v_{\mathrm{ext}}(\mathbf{r}, \omega)+\int \mathrm{d} \mathbf{r}^{\prime}\left[\frac{1}{\left|\mathbf{r}-\mathbf{r}^{\prime}\right|}+f_{\mathrm{xc}}\left(\mathbf{r}, \mathbf{r}^{\prime}, \omega\right)\right] \delta \rho\left(\mathbf{r}^{\prime}, \omega\right)
$$

Here, $f_{\mathrm{xc}}\left([\rho] ; \mathbf{r}, \mathbf{r}^{\prime}, \omega\right)$ is the Fourier transform of the exchange-correlation kernel:

$$
f_{\mathrm{xc}}\left([\rho] ; \mathbf{r}, \mathbf{r}^{\prime}, t-t^{\prime}\right)=\frac{\delta v_{\mathrm{xc}}([\rho] ; \mathbf{r}, t)}{\delta \rho\left(\mathbf{r}^{\prime}, t^{\prime}\right)} .
$$

By combining Eqs. 2.32-2.34, we can derive a Dyson-like equation for the response function

$$
\begin{aligned}
\chi\left(\mathbf{r}, \mathbf{r}^{\prime}, \omega\right) & =\chi_{\mathrm{KS}}\left(\mathbf{r}, \mathbf{r}^{\prime}, \omega\right) \\
& +\int \mathrm{d} \mathbf{x} \int \mathrm{d} \mathbf{x}^{\prime} \chi(\mathbf{r}, \mathbf{x}, \omega)\left[\frac{1}{\left|\mathbf{x}-\mathbf{x}^{\prime}\right|}+f_{\mathrm{xc}}\left(\mathbf{x}, \mathbf{x}^{\prime}, \omega\right)\right] \chi_{\mathrm{KS}}\left(\mathbf{x}^{\prime}, \mathbf{r}^{\prime}, \omega\right) .
\end{aligned}
$$

The response $\chi$ of the interacting system can be obtained via a self-consistent solution if the exact exchange-correlation kernel is known. However, achieving a complete numerical solution of this equation is rather difficult. Instead, we make use of 
the knowledge that the excitation energies of the interacting system correspond to the poles of the density response function $\chi$. In the same manner, the Kohn-Sham response function $\chi_{\mathrm{KS}}$ has poles that coincide with the non-interacting excitation energies, which are given by the difference of Kohn-Sham eigenvalues.

By employing a sequence of algebraic manipulations, linear-response TDDFT can be expressed in the form of the so-called Casida's equations [42], which is the form used in most quantum chemistry codes. There, the poles of the the response functions, $\Omega=E_{m}-E_{0}$, are obtained as solutions of a non-Hermitian eigenvalue problem:

$$
\left[\begin{array}{ll}
\mathbf{A} & \mathbf{B} \\
\mathbf{B} & \mathbf{A}
\end{array}\right]\left(\begin{array}{c}
\vec{X} \\
\vec{Y}
\end{array}\right)=\Omega\left[\begin{array}{cc}
-1 & 0 \\
\mathbf{0} & \mathbf{1}
\end{array}\right]\left(\begin{array}{c}
\vec{X} \\
\vec{Y}
\end{array}\right),
$$

where the matrices $\mathbf{A}$ and $\mathbf{B}$ are defined as:

$$
\begin{aligned}
& A_{i a, i^{\prime} a^{\prime}}=\delta_{i i^{\prime}} \delta_{a a^{\prime}}\left(\epsilon_{a}-\epsilon_{i}\right)+K_{i a, i^{\prime} a^{\prime}} \\
& B_{i a, i^{\prime} a^{\prime}}=K_{i a, a^{\prime} i^{\prime}}=\left(i a\left|\frac{1}{\left|\mathbf{r}-\mathbf{r}^{\prime}\right|}\right| a^{\prime} i^{\prime}\right)+\left(i a\left|f_{\mathrm{xc}}\right| a^{\prime} i^{\prime}\right) .
\end{aligned}
$$

The excitation energies are given by the eigenvalues, while the oscillator strengths can be computed from the eigenvectors.

TDDFT is a very appealing method for obtaining the excited-state properties of large molecular systems as it has a rather favorable scaling of approximately $\mathcal{O}\left(N^{3}\right)$ and is often fairly accurate. Nevertheless, it is important to note that approximate linear-response TDDFT does have limits [39]. In particular, it only contains dressed one-electron excitations, which is a problem if the system under study displays excited states with strong multi-configurational character. Another well-known issue is the underestimation of charge-transfer excitations [43-45], which can however be largely cured by the use of range-separated functionals.

\subsubsection{Quantum Monte Carlo Methods}

In our excited-state studies, we will employ the variational (VMC) and diffusion (DMC) Monte Carlo methods, which are the most commonly employed flavors of continuous quantum Monte Carlo (QMC) [46]. These methods are also wave function based but, at variance with conventional quantum chemistry, provide an approximate solution of the Schrödinger equation stochastically. Since the integrals are not computed analytically, QMC offers a lot of freedom in the choice of the functional form of the many-body wave function, which can depend explicitly on the electronelectron distances. The VMC and DMC approaches exhibit favorable scaling with the number of electrons, namely, $N^{4}$ as compared for instance to $N^{7}$ of the coupled cluster singles and doubles with perturbative triples $[\mathrm{CCSD}(\mathrm{T})]$ method.

\section{Variational Monte Carlo}

The variational Monte Carlo method is the simplest flavor of QMC and uses Monte Carlo techniques to evaluate the expectation value of an operator on a given wave 
function. For instance, let us consider computing the expectation value of the Hamiltonian $\mathcal{H}$ on a given trial wave function $\Psi_{\mathrm{T}}$ :

$$
E_{V}=\frac{\int \Psi_{\mathrm{T}}^{*}(\mathbf{R}) \mathcal{H} \Psi_{\mathrm{T}}(\mathbf{R}) d \mathbf{R}}{\int \Psi_{\mathrm{T}}^{*}(\mathbf{R}) \Psi_{\mathrm{T}}(\mathbf{R}) \mathrm{d} \mathbf{R}},
$$

where we denote with $\mathbf{R}$ the $3 \mathrm{~N}$ electron coordinates. This expectation value can be rewritten as

$$
E_{V}=\frac{\int\left|\Psi_{\mathrm{T}}(\mathbf{R})\right|^{2}\left[\Psi_{\mathrm{T}}(\mathbf{R})^{-1} \mathcal{H} \Psi_{\mathrm{T}}(\mathbf{R})\right] d \mathbf{R}}{\int\left|\Psi_{\mathrm{T}}(\mathbf{R})\right|^{2} d \mathbf{R}}=\int \rho(\mathbf{R}) E_{L}(\mathbf{R}) \mathrm{d} \mathbf{R},
$$

where

$$
\rho(\mathbf{R})=\frac{\left|\Psi_{\mathrm{T}}(\mathbf{R})\right|^{2}}{\int\left|\Psi_{\mathrm{T}}(\mathbf{R})\right|^{2} \mathrm{~d} \mathbf{R}},
$$

and the local energy is defined as

$$
E_{L}(\mathbf{R})=\Psi_{\mathrm{T}}(\mathbf{R})^{-1} \mathcal{H} \Psi_{\mathrm{T}}(\mathbf{R}),
$$

Since $\rho(\mathbf{R})$ is a positive quantity and integrates to 1 , we can interpret it as a probability distribution and use classical Monte Carlo techniques to sample a set of configurations $\left\{\mathbf{R}_{m}\right\}$ distributed according to $\rho(\mathbf{R})$. The expectation value can then be estimated as an average of the local energy $E_{L}(\mathbf{R})$ evaluated on these configurations:

$$
E_{V} \approx \frac{1}{M} \sum_{m=1}^{M} E_{L}\left(\mathbf{R}_{m}\right)
$$

Note that in this derivation, we can substitute the Hamiltonian $\mathcal{H}$ with any operator $\mathcal{O}$ diagonal in space representation.

For a realistic molecular system, the square of the many-body wave function is a complicated probability distribution in a high-dimensional space, whose normalization is usually not easy to compute. Therefore, we cannot use direct sampling techniques but we employ the classical Metropolis algorithm [47] to generate a sequence of configurations $\left\{\mathbf{R}_{m}\right\}$ distributed according to $\rho(\mathbf{R})$. The Metropolis algorithm is a general method to sample an arbitrary probability distribution without knowing its normalization. Here, we employ the VMC algorithm as described in Ref. 48, which uses a non-symmetrical transition matrix in the Metropolis algorithm, and which we properly modified to deal with pseudopotentials.

The functional form of our trial wavefunction is of the Jastrow-Slater form, that is, a conventional CI expansion multiplied by the so-called Jastrow factor:

$$
\Psi\left(\mathbf{r}_{1}, \ldots, \mathbf{r}_{N}\right)=\mathcal{J}\left(\mathbf{r}_{1}, \ldots, \mathbf{r}_{N}\right) \sum_{k} d_{k} D_{k}^{\uparrow}\left(\mathbf{r}_{1}, \ldots, \mathbf{r}_{N_{\uparrow}}\right) D_{k}^{\downarrow}\left(\mathbf{r}_{N_{\uparrow}+1}, \ldots, \mathbf{r}_{N}\right),
$$

where $D_{k}^{\uparrow}$ and $D_{k}^{\downarrow}$ are Slater determinants of single particle orbitals for the up and down spin electrons, respectively, and the Jastrow factor ensures the Kato cusp conditions, i.e., a proper discontinuity of the derivatives of the wave function at the 
coalescence points (points where the position of two electrons coincide). For most applications, we use the following form for the Jastrow factor:

$$
\mathcal{J}\left(\mathbf{r}_{1}, \ldots, \mathbf{r}_{N}\right)=\prod_{\alpha, i} \exp \left\{A\left(r_{i \alpha}\right)\right\} \times \prod_{i<j} \exp \left\{B\left(r_{i j}\right)\right\}
$$

in which $A\left(r_{i \alpha}\right)$ depends on the electron-nucleus distances and $B\left(r_{i j}\right)$ on the electron-electron distances. Both functions are fifth-order polynomials of scaled distances $\bar{r}=\frac{1-e^{\kappa r}}{\kappa}$, and different polynomial parameters are used for different atom types in the case of the electron-nucleus terms. We also include additional electronelectron-nucleus "three-body" terms in the Jastrow factor to test the quality of the wave function for the particular systems studied in Chapter 4.

The quality of the trial wave function affects the statistical efficiency of the QMC algorithms as well as the final accuracy of the results also in DMC. The parameters in the Jastrow factor must therefore be optimized together with the parameters in the determinantal component (CI and LCAO coefficients) since the optimal values will be different from the initial DFT or MCSCF ones, which have been obtained in the absence of the Jastrow factor. For the optimization of the parameters in the trial wave function of a system in its ground state, we use the so-called linear optimization method within energy minimization [49]. Since we are also interested in excited states, we want to be able to optimize the parameters of the multiple (ground and excited) orthogonal states described by the wave functions:

$$
\Psi_{I}=\sum_{i=1}^{N_{\mathrm{CSF}}} c_{i}^{I} \mathcal{J} C_{i} .
$$

which share the same Jastrow factor and orbitals but different linear coefficients. To this end, we employ a generalization of the linear method to state-average (SA) optimization [50] to determine a set of orbitals and a Jastrow factor which give a comparably good description of the states under considerations while preserving orthogonality among the states through the CI coefficients.

\section{Diffusion Monte Carlo}

VMC is a very effective approach to explore which type of correlation is important in the description of the system of interest and to construct a high-quality wave function via optimization of its parameters. It is then possible to improve on this wave function by employing other flavors of QMC, which go under the general name of projector Monte Carlo techniques. The idea of projection Monte Carlo is to construct an operator that inverts the spectrum of $\mathcal{H}$ and to project out all excited states, thereby obtaining the ground state of $\mathcal{H}$, by repeated application of this operator on an initial trial state. Diffusion Monte Carlo (DMC) and full CI QMC (FCIQMC) are both examples of this approach, and will be described here.

In DMC, the projection operator is given by $\exp \left[-\tau\left(\mathcal{H}-E_{\mathrm{T}}\right)\right]$, and is applied to an initial trial wave function to obtain the sequence of wave functions:

$$
\Psi^{(n)}=e^{-\tau\left(\mathcal{H}-E_{\mathrm{T}}\right)} \Psi^{(n-1)} .
$$


If we expand the initial wave function $\Psi^{(0)}$ on the eigenstates $\Psi_{i}$ with energies $E_{i}$ of $\mathcal{H}$, we obtain for $\Psi^{(n)}$ :

$$
\Psi^{(n)}=\sum_{i} \Psi_{i}\left\langle\Psi^{(0)} \mid \Psi_{i}\right\rangle e^{-n \tau\left(E_{i}-E_{\mathrm{T}}\right)},
$$

Since the coefficients of the excited states die off exponentially fast relative to the one of the ground state, we obtain:

$$
\lim _{n \rightarrow \infty} \Psi^{(n)}=\Psi_{0}\left\langle\Psi^{(0)} \mid \Psi_{0}\right\rangle e^{-n \tau\left(E_{0}-E_{\mathrm{T}}\right)} .
$$

Therefore, if we adjust the trial energy $E_{\mathrm{T}} \approx E_{0}$ to keep the overall normalization of $\Psi^{(n)}$ fixed, the projection gives us the ground state $\Psi_{0}$ of the Hamiltonian.

To perform a stochastic realization of the formulas above, we need to choose a basis set to express these equations. In DMC, one works in real space and rewrite Eq. 2.46 in integral form as:

$$
\Psi^{(n)}\left(\mathbf{R}^{\prime}, t+\tau\right)=\int \mathrm{d} \mathbf{R} G\left(\mathbf{R}^{\prime}, \mathbf{R}, \tau\right) \Psi^{(n-1)}(\mathbf{R}, t),
$$

where the Green's function is defined as:

$$
G\left(\mathbf{R}^{\prime}, \mathbf{R}, \tau\right)=\left\langle\mathbf{R}^{\prime}\left|e^{-\tau\left(\mathcal{H}-E_{\mathrm{T}}\right)}\right| \mathbf{R}\right\rangle .
$$

This integral is computed stochastically by sampling the trial wave function and the Green's function in Eq. 2.49 (for the moment, let us assume that the wave function is positive so that we can interpret it as a probability distribution). For small $\tau$, the Green's function can be approximated via the Trotter-Suzuki formula as:

$$
\left\langle\mathbf{R}^{\prime}\left|e^{-\mathcal{H} \tau}\right| \mathbf{R}\right\rangle \approx \frac{1}{(2 \pi \tau)^{3 N / 2}} \exp \left[-\frac{\left(\mathbf{R}^{\prime}-\mathbf{R}\right)^{2}}{2 \tau}\right] \exp [-\tau \mathcal{V}(\mathbf{R})] .
$$

Therefore, the iteration in Eq. 2.49 can be interpreted as a branching random walk, where the walkers perform a diffusion step and their weights are then rescaled by the positive exponential factor. The results of a DMC calculation must then be extrapolated for $\tau$ which goes to zero.

Since the potential can vary significantly in configuration space and even be unbounded, the approach is inefficient but it is possible to overcome this problem by using the so-called importance sampling algorithm [51] and exploiting the trial wave function $\Psi$ constructed in VMC. To do so, we start from Eq. 2.49, multiply each side by $\Psi$, and define the probability distribution $f^{(n)}(\mathbf{R})=\Psi(\mathbf{R}) \Psi^{(n)}(\mathbf{R})$ which satisfies

$$
f^{(n)}\left(\mathbf{R}^{\prime}, t+\tau\right)=\int \mathrm{d} \mathbf{R} \tilde{G}\left(\mathbf{R}^{\prime}, \mathbf{R}, \tau\right) f^{(n-1)}(\mathbf{R}, t) .
$$

The importance-sampled Green's function is given by:

$$
\tilde{G}\left(\mathbf{R}^{\prime}, \mathbf{R}, \tau\right)=\Psi\left(\mathbf{R}^{\prime}\right)\left\langle\mathbf{R}^{\prime}\left|e^{-\tau\left(\mathcal{H}-E_{\mathrm{T}}\right)}\right| \mathbf{R}\right\rangle / \Psi(\mathbf{R}) .
$$


For small $\tau$, the resulting drift-diffusion-branching short-time Green's function is given by:

$$
\begin{array}{r}
\tilde{G}\left(\mathbf{R}^{\prime}, \mathbf{R}, \tau\right)=(2 \pi \tau)^{3 N / 2} \exp \left[-\frac{\left(\mathbf{R}^{\prime}-\mathbf{R}-\tau \mathbf{V}(\mathbf{R})\right)^{2}}{2 \tau}\right] \times \\
\quad \times \exp \left\{-\tau\left[\left(E_{\mathrm{L}}(\mathbf{R})+E_{\mathrm{L}}\left(\mathbf{R}^{\prime}\right)\right) / 2-E_{\mathrm{T}}\right]\right\}+O\left(\tau^{2}\right) .
\end{array}
$$

This Green's function offers the advantage that the quantum velocity pushes the walkers towards regions where the wave function is large and that the local energy $E_{\mathrm{L}}(\mathbf{R})$ instead of the potential $\mathcal{V}(\mathbf{R})$ appears in the branching factor. A good trial wave function will result in smaller fluctuations in the branching factor and the instabilities due to the divergences of the Coulomb potential are removed since the wave function obeys the cusp conditions.

Unfortunately, electrons are fermions and the trial wave function must be antisymmetric, so it cannot be interpreted as a probability distribution. Algorithms which attempt to handle both signs of the wave function lead to the so-called fermion sign problem: The bosonic component grows at the expense of the fermionic one, and the antisymmetric signal is lost in the noise. To avoid this problem, we work in the so-called fixed-node approximation and solve the evolution equation with the boundary condition that the solution has the same nodes (the same zeros) as the given trial wave function. The Schrödinger equation is solved exactly inside the nodal regions but not at the nodes where the solution will have a discontinuity of the derivatives. The fixed-node solution is exact only if the nodes of the trial wave function are exact and, for approximate nodes, only gives an upper bound to the exact energy.

Importantly, DMC can also be used to study the excited states of a given Hamiltonian. If the state is the lowest state of a one-dimensional representation of the point group of the molecules, DMC will yield a solution which is variational. If the excited state is not the lowest state in its symmetry, we can use DMC in the fixed-node approximation and employ the nodes of a trial (excited-state) wave function to keep the solution from collapsing on the ground state. However, we do not have a variational principle on the excitation energy and are only guaranteed to obtain the exact solution in the limit of exact nodes of the trial wave function.

\section{Full Configuration Interaction Quantum Monte Carlo}

Full CI quantum Monte Carlo (FCIQMC) [52] is also a projection Monte Carlo method and uses the same projection operator as DMC but the equations are expressed in the Slater determinant space and not in real space. If we express the wave function in the basis of Slater determinants, $D_{i}$, generated by arranging the electrons in a given set of spin orbitals, we obtain the FCI representation of the wave function,

$$
\Psi=\sum_{i} c_{i} D_{i}
$$

The projection (Eq. 2.46) formulated in this determinantal basis is then equivalent to a set of coupled equations for the evolution of the determinant coefficients in the CI 
expansion as:

$$
-\frac{d c_{i}}{d \tau}=\left(H_{i i}-E_{\mathrm{T}}\right) c_{i}+\sum_{j \neq i} H_{i j} c_{j},
$$

where $H_{i j}$ are the matrix elements of the Hamiltonian between the Slater determinants.

Walkers in FCIQMC therefore live in determinant space and, at a given time, the CI coefficient of a certain determinant will be proportional to the number of walkers occupying it, $c_{i} \propto \sum_{\alpha_{i}} s_{\alpha}$, where $s_{\alpha}= \pm 1$ is the sign of a given walker. The wave function will evolve in time by walkers spawning new walkers onto other determinants (off-diagonal terms) and dying or cloning (diagonal terms) depending on the trial energy. The wave function will evolve to the ground-state solution in the limit of very large walker populations or by averaging the CI coefficients over a sufficiently long amount of time. The ground-state energy can be estimated either as the average trial energy $E_{\mathrm{T}}$ or through the so-called mixed estimator,

$$
E_{\text {proj }}=\frac{\left\langle D_{0}|\mathcal{H}| \Psi_{\mathrm{FCI}}\right\rangle}{\left\langle D_{0} \mid \Psi_{\mathrm{FCI}}\right\rangle}=\sum_{j}\left\langle D_{0}|\mathcal{H}| D_{j}\right\rangle \frac{\left\langle N_{j}\right\rangle}{\left\langle N_{0}\right\rangle},
$$

which will generally have better statistical behavior, especially if a good trial wave function (in place of $D_{0}$ ) is used.

Even though FCIQMC still suffers from exponential scaling, the exponent has a very small prefactor and, for a given system, the number of walkers needed to converge a FCIQMC calculation is several orders of magnitude smaller than the original full CI problem. Owing to the use of determinant space, the FCIQMC wave function is guaranteed to be fermionic, unlike the DMC one, but the method is still vulnerable to a different sort of sign problem, namely, that $|\Psi\rangle$ and $-|\Psi\rangle$ are both viable solutions. In practical calculations, the FCIQMC method is stabilized by introducing the so-called initiator approach [53], which allows only determinants with a sufficient number of walkers with the same sign (and therefore likely to possess a relatively sure sign) to spawn progeny on new determinants. Initiator FCIQMC suffers however from a bias and extrapolations with the size of the walker population must be performed. Other recent improvements on FCIQMC include a semi-stochastic propagation [54], where the propagation on a small part of the determinant space is performed deterministically and not stochastically.

In this thesis, we also consider excited states using FCIQMC but only in the lowest state of a particular symmetry. Consequently, these states are "ground states" in their symmetry class and the application of FCIQMC to their study is therefore straightforward. Algorithms to treat non-trivial excited states have also been recently proposed [55].

\subsection{Multiscale Techniques}

In this thesis, we investigate light absorption in complex systems comprising as many as thousands of atoms, for which a full quantum mechanical treatment is clearly im- 
possible. Fortunately, the primary photoabsorption process often involves a photosensitive component (the chromophore) and few surrounding residues, and is therefore spatially localized. Therefore, it is not unreasonable to attempt to partition the system into a smaller quantum subsystem and a larger environment to be simulated by less expensive classical methods, that is, either point charges or point charges and polarizable dipoles. These hybrid methods are called quantum mechanics in molecular mechanics (QM/MM) [56] approaches.

Another route is to treat the environment (or a part thereof) at the DFT level. This approach has the advantage that a large region surrounding the chromophore can be treated within DFT compared to what would be possible with highly correlated calculations, while being in principle superior to QM/MM owing to a more faithful, quantum mechanical description of the environment and its coupling to the embedded region.

\subsubsection{Quantum Mechanics in Molecular Mechanics Tech- niques}

Classical MM calculations employ empirical force fields to describe interactions of the atoms [57]. The chosen force field defines the functional form of the potentialenergy function and the parameters set for the atoms. The potential contains bonded terms, for example, bond stretching, bending of angles, and torsional angles as well as non-bonded contributions, which are van der Waals and the Coulomb interaction terms between the partial point charges of the atoms.

In Chapter 5, we perform ab initio molecular dynamics simulations of GFP using a QM/MM framework [58, 59] as implemented in the CPMD cose [60]. The dynamics of the chromophore is governed by DFT calculations via the Car-Parrinello approach [61], while the rest of the protein is described by a classical force field. We then extract snapshots from the QM/MM trajectory and perform single-point calculations of the excited-state properties of the system, where the the chromophore (or a slightly larger system) is treated quantum mechanically in the presence of an electrostatic potential created by the point charges of the environment:

$$
H=H_{\mathrm{QM}}+\sum_{a} q_{a} \hat{V}_{\mathrm{QM}}\left(\mathbf{r}_{a}\right)
$$

where $a$ runs over the atoms in the environment and $\hat{V}_{\mathrm{QM}}\left(\mathbf{r}_{a}\right)=\sum_{i} \frac{1}{\left|\mathbf{r}_{a}-\hat{\mathbf{r}}_{i}\right|}$ is the electrostatic potential operator due to the electrons of the embedded species.

Since the QM/MM boundary in our system cuts through chemical bonds of the protein, we "cap" each broken bond with a hydrogen atom, which is seen by the QM system but not by the MM atoms. In the single-point calculations, we set to zero the few MM charges closest to the QM/MM boundary to avoid problems of overpolarization and redistribute them to the nearest $\mathrm{MM}$ atoms of the same amino acid to keep its total charge constant. Finally, in standard wave function and (TD)DFT calculations, one computes the matrix elements of the external potential due to the charges on a given basis set only at the beginning of the run. In quantum Monte 
Carlo, since one must repeatedly evaluate the potential along the random walk for different positions of the electrons, we store the potential due to the point charges on a grid and, when needed, compute it by interpolatation using polynomials.

\subsubsection{QM/MMpol formalism}

A more sophisticated, yet classical, description of the environment can be obtained by including polarizable dipoles along with the static charges in a so-called QM/MMpol scheme. The use of polarizable dipoles is not computationally much more expensive and allows one to polarize the environment to the ground state of the active site for an enhanced description of its surroundings. Furthermore, it is possible to include the response of the protein to the excitation of the embedded region by polarizing the dipoles with respect to the excited chromophore. The resulting improvement in the description of the excited state of the embedded species can be significant and is not known a priori. On electrostatic grounds, one might expect that if the ground state has a large dipole moment (or even a charge), a correct description of the ground-state polarization is surely important, while if the change in dipole moment between the excited and the ground states is large, then including the response of the dipoles to the excitation will be also a crucial factor. We will analyze the validity of this principle in Chapter 5 for GFP.

In the QM/MMpol framework, the environment will be modeled as a set of sites with charge $q_{\mathrm{a}}$ and polarizability $\alpha_{\mathrm{a}}$, assumed here to be isotropic. For a given wave function $\Psi$, the induced dipoles in equilibrium with the quantum system are given by:

$$
\begin{aligned}
\boldsymbol{\mu}_{\mathrm{a}} & =\alpha_{\mathrm{a}} \mathbf{E}\left(\mathbf{r}_{\mathrm{a}}\right) \\
& =\alpha_{\mathrm{a}}\left[\left\langle\Psi\left|\hat{\mathbf{E}}_{\mathrm{QM}}\left(\mathbf{r}_{\mathrm{a}}\right)\right| \Psi\right\rangle+\sum_{\mathrm{b} \neq \mathrm{a}} \frac{q_{\mathrm{b}}\left(\mathbf{r}_{\mathrm{a}}-\mathbf{r}_{\mathrm{m}}\right)}{\left|\mathbf{r}_{\mathrm{a}}-\mathbf{r}_{\mathrm{m}}\right|^{2}}-\mathbf{T}_{\mathrm{ab}} \cdot \boldsymbol{\mu}_{\mathrm{b}}\right],
\end{aligned}
$$

where $\mathbf{E}$ is the total electric field at site $a$ which is generated by the quantum system (electrons and nuclei), the static charges, and the other dipoles. The interaction tensor $\mathrm{T}$ is equal to:

$$
\left(\mathbf{T}_{\mathrm{ab}}\right)_{i j}=\frac{1}{r_{\mathrm{ab}}^{3}}\left[\delta_{i j}-3 \frac{\left(\mathbf{r}_{a b}\right)_{i}\left(\mathbf{r}_{\mathrm{ab}}\right)_{j}}{r_{\mathrm{ab}}^{2}}\right] .
$$

We note that, in practical calculations, the sum over $b$ in Eq. 2.59 does not run over the sites within the same molecule or too close to dipole $a$ for the sake of numerical stability.

The wave function $\Psi$ obeys the Schrödinger equation:

$$
\left(\mathcal{H}_{\mathrm{QM}}+\mathcal{H}_{\mathrm{QM} / \mathrm{MM}}\right) \Psi=E \Psi
$$

in which $\mathcal{H}_{\mathrm{QM}}$ is the Hamiltonian of the isolated quantum system and

$$
\begin{aligned}
\mathcal{H}_{\mathrm{QM} / \mathrm{MM}} & =\mathcal{H}_{\text {solute }, \mathrm{q}}+\mathcal{H}_{\text {solute }, \mu} \\
& =\sum_{\mathrm{a}} q_{\mathrm{a}} \hat{V}_{\mathrm{QM}}\left(\mathbf{r}_{\mathrm{a}}\right)-\sum_{\mathrm{a}} \mu_{\mathrm{a}} \cdot \hat{\mathbf{E}}_{\mathrm{QM}}\left(\mathbf{r}_{\mathrm{a}}\right),
\end{aligned}
$$


which describes the interaction between the potential and the field of the electrons and nuclei with the MM charges and dipoles. Since the dipoles depend on the wave function, one solves a non-linear Schrödinger equation:

$$
\left[\mathcal{H}_{\mathrm{QM}}+\sum_{\mathrm{a}} q_{\mathrm{a}} \hat{V}_{\mathrm{QM}}\left(\mathbf{r}_{\mathrm{a}}\right)+V(\Psi)\right]|\Psi\rangle=E|\Psi\rangle
$$

where the potential depends on the wave function quadratically through the dipoles. Therefore, this equation must be solved self-consistently with the ones defining the dipoles (Eq. 2.59) and this procedure yields different potentials for the ground and the excited states of interest, with wave functions which will therefore be nonorthogonal.

The total energy of the system must also include the MM energy as a shift in the energy eigenvalue of the QM system, accounting for charge-charge, charge-dipole, dipole-dipole and self-energy contributions:

$$
E_{\mathrm{MM}}=U_{q, q}+U_{q, \mu}+U_{\mu, \mu}+U_{\text {self }} .
$$

The self-energy is the work exerted by the electric field to induce the dipole $\mu_{a}$ at site a with isotropic polarizability $\alpha_{\mathrm{a}}$ and is given by

$$
U_{\text {self }}=\frac{1}{2} \sum_{\mathrm{a}} \frac{\mu_{\mathrm{a}}^{2}}{\alpha_{\mathrm{a}}}
$$

If the dipoles are in equilibrium with the given wave function, Eq. 2.59 holds and the self-energy cancels half of the interaction of the dipoles with the rest of the system.

Alternatively, one introduces the so-called free-energy operator:

$$
\hat{G}(\Psi)=H_{\mathrm{QM}}+\sum_{a} \hat{V}_{\mathrm{QM}}\left(\mathbf{r}_{\mathrm{a}}\right)+\frac{1}{2} V(\psi) .
$$

whose expectation value yields the total energy of the system with dipoles in equilibrium with the quantum part, and obtain the Schrödinger equation (Eq. 2.63) from the variational principle:

$$
\delta \frac{\langle\Psi|\hat{G}(\psi)| \Psi\rangle}{\langle\Psi \mid \Psi\rangle}=0
$$

using that $\langle\Psi|V(\Psi)| \Psi\rangle$ depends quadratically on $\Psi^{1}$.

It is possible to follow several routes to compute the excited states of a quantum system embedded in polarizable dipoles. The first approximation neglects polarization altogether (denoted as "nopol") and simply corresponds to an embedding calculation in static point charges as in the previous subsection. The next possibility is to polarize the dipoles to the ground state of the quantum system (polGS) and to then freeze the dipole values in the calculation of the excited states. Finally, one can allow the environment to respond to the electronic excitation of the embedded

\footnotetext{
${ }^{1}$ In the more general case where $V(\Psi) \propto\left(\Psi^{*} \Psi\right)^{n}$, the potential in the free-energy operator is instead scaled by $1 / \mathrm{n}[62]$.
} 
species. While the formulation of the first two approximations (nopol and polGS) is straightforward for any quantum method, there are two possible ways of including responsive dipoles.

In the so-called state-specific formulation (polSS), we solve self-consistently the equations (Eqs. 2.59 and 2.63) for the ground and the excited states of interest. The dipoles respond to the change in electrostatic potential created by the wave function upon excitation and the classical and quantum domains are seperately brought into equilibrium in the ground and the excited states. The second formulation (polLR) is based on linear-response theory and determines the excitation energies as poles in the dynamic polarizability of the system. Its derivation relies on the time-dependent Dirac-Frenkel variational principle for the free-energy operator $\hat{G}(\Psi)$, an extension of Eq. 2.67 yielding the time-dependent Schrödinger equation corresponding to the time-independent Eq. 2.63. For isolated quantum calculations (with potentials which are linear in the wave function), state-specific and linear-response-based approach give identical excitations but the two routes for the computation of excited states (polSS and polLR) in the quadratic potential $V(\Psi)$ can yield significantly different excitation energies, even in the limit of the exact solutions of the Schrödinger equation (Eq. 2.63) An anlysis using first-order perturbation theory reveals that the polSS and polLR excitation energies behave as [63]:

$$
\frac{\Delta E_{\mathrm{polSS}}-\Delta E_{\mathrm{polGS}}}{\Delta E_{\mathrm{polLR}}-\Delta E_{\mathrm{polGS}}} \approx \frac{\left|\boldsymbol{\mu}_{1}-\boldsymbol{\mu}_{0}\right|^{2}}{2 \mu_{01}^{2}},
$$

where $\boldsymbol{\mu}_{I}=\left\langle\Psi_{I}\left|\sum_{i} \hat{\mathbf{r}}_{i}\right| \Psi_{I}\right\rangle$ is the expected value of the electronic dipole moment in a particular state and $\mu_{01}=\left\langle\Psi_{0}\left|\sum_{i} \hat{\mathbf{r}}_{i}\right| \Psi_{1}\right\rangle$ the transition dipole moment (TDM) of the excitation. For a given system and excitation, the change in dipole moment and the TDM can have sizable differences. The excitation-energy shift yielded by polSS is readily understood from electrostatics arguments and is what one might expect to recover from classical embedding. The polLR contribution has a somewhat less intuitive physical meaning and has been shown to correspond to a dispersion-like coupling between the quantum oscillations of the excitation and the classical dipoles in the environment [64].

Finally, we note that, as parameters for the polarizable dipoles, one can use standard polarixable force-field parameters or obtain them through ab initio calculations [65], but thereby possibly significantly increasing the computational cost.

\subsubsection{DFT embedding}

In DFT embedding, one treats the embedded system and its excitation with a highlevel wave function method, while describing the rest of the system with the cheaper, yet quantum mechanical, DFT method. This combination allows a proper description of the Pauli repulsion of the active site with the immediate surroundings and carries the advantage of being $a b$ initio and, potentially, more accurate.

DFT embedding is based on so-called subsystem DFT, where we partition our 
system in two subsystems ${ }^{2}$, each containing a given set of atoms and a fixed number of electrons. If we partition the density as $\rho_{\text {tot }}(\mathbf{r})=\rho_{1}(\mathbf{r})+\rho_{2}(\mathbf{r})$ and the number of electrons as a sum of two constant integers, $N_{\text {tot }}=N_{1}+N_{2}$, we can rewrite the total energy as:

$$
E_{\text {tot }}\left[\rho_{1}+\rho_{2}\right]=E_{1}\left[\rho_{1}\right]+E_{2}\left[\rho_{2}\right]+E_{\text {int }}\left[\rho_{1}, \rho_{2}\right]
$$

where $E_{I}\left[\rho_{I}\right]$ is the Kohn-Sham energy of the isolated system $I$ (Eq. 2.19) and the interaction energy is given by

$$
E_{\text {int }}\left[\rho_{1}, \rho_{2}\right]=J\left[\rho_{1}, \rho_{2}\right]+V_{\text {nuc1 } 1}\left[\rho_{2}\right]+V_{\text {nuc } 2}\left[\rho_{1}\right]+F^{\text {nadd }}\left[\rho_{1}, \rho_{2}\right],
$$

where $J\left[\rho_{1}, \rho_{2}\right]$ is the inter-subsystem electron-electron repulsion, $V_{\text {nucI }}\left[\rho_{J}\right]$ the interaction of the nuclei of system $I$ with the density of system $J$, and the nonadditive contribution is given by:

$$
\begin{aligned}
F_{\text {nadd }}\left[\rho_{1}, \rho_{2}\right] & =T_{s}\left[\rho_{1}+\rho_{2}\right]-\left(T_{s}\left[\rho_{1}\right]+T_{s}\left[\rho_{2}\right]\right) \\
& +E_{x c}\left[\rho_{1}+\rho_{2}\right]-\left(E_{x c}\left[\rho_{1}\right]+E_{x c}\left[\rho_{2}\right]\right) .
\end{aligned}
$$

and is generally different than zero since both $T_{s}$ and $E_{x c}$ are non-linear functional of the density. In particular, the kinetic contribution to $F_{\text {nadd }}$ is non zero since orbitals belonging to different subsystems are generally not orthogonal, and is approximated using an orbital-free kinetic energy functional. Furthermore, for the exchangecorrelation terms in $F_{\text {nadd }}$, we need to use an orbital-free approximate functional (e.g. LDA or GGA but not an hybrid) since the computation of $E_{x c}$ for the complete system would otherwise require the knowledge of the corresponding Kohn-Sham orbitals.

Differentiation of Eq. 2.69 with respect to the density of subsystem 1 will result in a modified Kohn-Sham equation:

$$
\left[-\frac{1}{2} \nabla^{2}+v_{\mathrm{eff}}\left(\left[\rho_{1}\right] ; \mathbf{r}\right)+v_{\mathrm{emb}}\left[\rho_{1}, \rho_{2}\right](\mathbf{r})\right] \phi_{i}=\epsilon_{i} \phi_{i}
$$

in which the embedding potential is the functional derivative of the interaction energy functional (Eq. 2.70):

$$
\begin{aligned}
v_{\mathrm{emb}}\left[\rho_{1}, \rho_{2}\right](\mathbf{r}) & =\frac{\delta E_{\text {int }}\left[\rho_{1}, \rho_{2}\right]}{\delta \rho_{1}(\mathbf{r})} \\
& =v_{\text {nuc } 2}(\mathbf{r})+\int \frac{\rho_{2}\left(\mathbf{r}^{\prime}\right)}{\left|\mathbf{r}-\mathbf{r}^{\prime}\right|} d \mathbf{r}^{\prime}+\frac{\delta F^{\text {nadd }}\left[\rho_{1}, \rho_{2}\right]}{\delta \rho_{1}(\mathbf{r})} .
\end{aligned}
$$

After solving these Kohn-Sham equations for subsystem 1 in the presence of the embedding potential, one may fix the newly obtained density for subsystem 1 to relax subsystem 2 , and continue in this way until self-consistency through a series of so-called "freeze\&thaw" cycles.

\footnotetext{
${ }^{2}$ The generalization to more than two subsystems is trivial but omitted here for simplicity.
} 
Wavefunction-in-DFT (WF/DFT) embedding is the generalization of this procedure where one obtains an embedding potential from a subsystem DFT calculation and uses it in a wave function calculation. In WF/DFT embedding, we express the energy of the full system as:

$$
E_{\text {tot }}\left[\Psi_{1}, \rho_{2}\right]=\left\langle\Psi_{1}\left|H_{1}\right| \Psi_{1}\right\rangle+E_{2}\left[\rho_{2}\right]+E_{\text {int }}\left[\rho_{1}, \rho_{2}\right]
$$

where the interaction energy term is still treated at the level of orbital-free DFT. When we minimize this energy expression with respect to $\Psi_{1}$, the resulting Schrödinger equation is

$$
\left[H_{1}+\sum_{i} v_{\mathrm{emb}}\left(\mathbf{r}_{i}\right)\right] \Psi=E \Psi
$$

which contains the same local embedding potential of Eq. 2.73. Through this procedure, we can obtain the ground state of the embedded system but can also compute the excited states in the same embedding potential, which can be viewed as describing a non-responsive or "frozen" environment. In this thesis, we introduce and test a new formulation of wavefunction-in-DFT embedding, in which the environment is allowed to respond to the excitation of the WF subsystem. This scheme results in different embedding potentials for the ground and excited states, much like in the case of MMpol described in the previous subsection. It is perhaps worth mentioning that the expression "response" should not be taken to express a temporal delay between the excitation of the active subsystem and the environmental relaxation, since both are changes in the electronic wave function and happen simultaneously. Details of our scheme can be found in Chapter 3 and 4 .

Finally, in Chapter 6, we present an embedding scheme which eliminates the need for an approximate kinetic-energy functional in the expression of the embedding potential, which is the main approximation of the method. However, at the time of writing, this approach has no rigorous way of treating environmental response since it is based on a ground-state potential reconstruction.

\subsection{Computational Details}

We list here the codes employed in the various calculations. Computational details specific to each calculation will be given in each chapter.

The Gromacs [66] code is a set of computational tools to perform and analyze classical molecular dynamics simulations.

The CPMD [60] code is used to perform the QM/MM calculation within DFT as QM method, while the Gromos [67] classical force field code is used for the MM part. We employ the QM/MM interface developed by Röthlisberger and coworkers [58, 59]. The CPMD code is a plane-wave/pseudopotential code particularly designed for ab-initio molecular dynamics.

The Molcas [68] code is a quantum chemistry code, based on Gaussian basis sets, that we use to perform CASSCF and CASPT2 calculations. 
The Gaussian 09 [69] code is a quantum chemistry code we use to perform ground-state DFT and linear-response TDDFT calculations. It uses Gaussian basis sets, and a wide range of exchange-correlation functionals is available.

The Amsterdam Density Functional ADF [70] code is a software package for first-principles electronic structure DFT calculations, using Slater functions for the construction of the orbitals. We employ this code to perform subsystem DFT calculations.

The GAMESS [71] code is a ab initio quantum chemistry package which we mostly use to generate the starting QMC wave functions either through a DFT or a SA-CASSCF calculation. This code employs Gaussian basis sets.

Other codes employed are the Dalton [72], Turbomole [73], and CFOUR [74] codes for coupled-cluster calculations, and the ORCA [75] code for NEVPT2 calculations. All of these codes employ Gaussian basis sets.

Finally, the code CHAMP is used for all the quantum Monte Carlo calculations. It can perform VMC and DMC calculations, and optimize the wave function parameters by energy minimization. 


\section{Bibliography}

[1] C. J. Cramer, Essentials of Computational Chemistry, 2nd ed. (Wiley, 2004).

[2] F. Jensen, Introduction to Computational Chemistry, 2nd ed. (Wiley, 2007).

[3] D. Roca-Sanjuán, F. Aquilante, and R. Lindh, WIREs Comput Mol Sc 2, 585 (2012).

[4] G. Ghigo, B. O. Roos, and P.-Å. Malmqvist, Chem. Phys. Lett. 396, 142 (2004).

[5] M. Schreiber, M. R. Silva-Junior, S. P. A. Sauer, and W. Thiel, J. Chem. Phys. 128, 134110 (2008).

[6] C. Angeli, M. Pastore, and R. Cimiraglia, Theor. Chem. Acc. 117, 743 (2007).

[7] G. D. Purvis and R. J. Bartlett, J. Chem. Phys. 76, 1910 (1982).

[8] K. Kowalski and P. Piecuch, J. Chem. Phys. 115, 643 (2001).

[9] S. A. Kucharski, M. Wloch, M. Musial, and R. J. Bartlett, J. Chem. Phys. 115, 8263 (2001).

[10] O. Christiansen, H. Koch, and P. Jørgensen, Chem. Phys. Lett. 243, 409 (1995).

[11] C. Hättig and F. Weigend, J. Chem. Phys. 113, 5154 (2000).

[12] C. Hättig, Adv. Quantum Chem. 50, 37 (2005).

[13] W. Koch and M. C. Holthausen, A Chemist's Guide to Density Functional Theory (Wiley-VCH, 2000).

[14] P. Hohenberg and W. Kohn, Phys. Rev. 136, B864 (1964).

[15] W. Kohn and L. J. Sham, Phys. Rev. 140, A1133 (1965).

[16] G. E. Scuseria and V. N. Staroverov, in Theory and Applications of Computational Chemistry - The First Forty Years, edited by C. E. Dykstra, G. Frenking, K. S. Kim, and G. E. Scuseria (Elsevier B.V, 2005), pp. 669-724.

[17] J. P. Perdew and K. Schmidt, AIP Conf. Proc. 577, 1 (2001).

[18] A. D. Becke, Phys. Rev. A 38, 3098 (1988). 
[19] C. Lee, W. Yang, and R. G. Parr, Phys. Rev. B 37, 785 (1988).

[20] J. P. Perdew, K. Burke, and M. Ernzerhof, Phys. Rev. Lett. 77, 3865 (1996).

[21] J. Perdew and Y. Wang, Phys. Rev. B 45, 13244 (1992).

[22] A. D. Becke, J. Chem. Phys. 98, 1372 (1993).

[23] J. P. Perdew, M. Ernzerhof, and K. Burke, J. Chem. Phys. 105, 9982 (1996).

[24] A. D. Becke, J. Chem. Phys. 98, 5648 (1993).

[25] P. J. Stephens, F. J. Devlin, C. F. Chabalowski, and M. J. Frisch, J. Phys. Chem. 98, 11623 (1994).

[26] Y. Zhao and D. G. Truhlar, J. Chem. Phys. 125, 194101 (2006).

[27] Y. Zhao and D. G. Truhlar, J. Phys. Chem. A 110, 13126 (2006).

[28] Y. Zhao and D. Truhlar, Theor. Chem. Acc. 120, 215 (2008).

[29] Y. Zhao and D. G. Truhlar, Acc. Chem. Res. 41, 157 (2008).

[30] K. A. Nguyen, P. N. Day, and R. Pachter, J. Chem. Phys. 135, 074109 (2011).

[31] H. Iikura, T. Tsuneda, T. Yanai, and K. Hirao, J. Chem. Phys. 115, 3540 (2001).

[32] T. Yanai, D. P. Tew, and N. C. Handy, Chem. Phys. Lett 393, 51 (2004).

[33] J. Heyd, G. E. Scuseria, and M. Ernzerhof, J. Chem. Phys. 118, 8207 (2003).

[34] L. A. Thomas, Proc. Camb. Phil. Soc. 23, 542 (1927).

[35] E. Fermi, Z. Phys. 48, 73 (1928).

[36] P. Cortona, Phys. Rev. B 44, 8454 (1991).

[37] A. Lembarki and H. Chermette, Phys. Rev. A 50, 5328 (1994).

[38] J. P. Perdew, Phys. Lett. A 165, 79 (1992).

[39] M. E. Casida, J. Mol. Struct. 914, 3 (2009).

[40] E. Runge and E. Gross, Phys. Rev. Lett. 52, 997 (1984).

[41] R. van Leeuwen, Phys. Rev. Lett. 82, 3863 (1998).

[42] M. E. Casida, in Recent Advances in Density Functional Methods, Part I, edited by D. P. Chong (World Scientific, 1995), p. 155.

[43] D. J. Tozer, R. D. Amos, N. C. Handy, B. O. Roos, and L. S. Andres, J. Mol. Phys. 97, 859 (1999). 
[44] M. E. Casida, F. Gutierrez, J. Guan, F. X. Gadea, and D. Salahub, J. Chem. Phys. 113, 7062 (2000).

[45] A. Dreuw, J. L. Weisman, and M. Head-Gordon, J. Chem. Phys. 119, 2943 (2003).

[46] A. Lüchow, WIREs Comput Mol Sc 1, 388 (2011).

[47] N. Metropolis, A. Rosenbluth, M. N. Rosenbluth, A. H. Teller, and E. Teller, J. Chem. Phys. 21, 1087 (1953).

[48] C. J. Umrigar, Phys. Rev. Lett. 71, 408 (1993).

[49] C. J. Umrigar, J. Toulouse, C. Filippi, S. Sorella, and R. G. Hennig, Phys. Rev. Lett. 98, 110201 (2007).

[50] C. Filippi, M. Zaccheddu, and F. Buda, J. Chem. Theory Comput. 5, 2074 (2009).

[51] D. M. Ceperley and B. J. Alder, Phys. Rev. Lett. 45, 566 (1980).

[52] G. H. Booth, A. J. W. Thom, and A. Alavi, J. Chem. Phys. 131, 054106 (2009).

[53] D. Cleland, G. H. Booth, and A. Alavi, J. Chem. Phys. 132, 041103 (2010).

[54] F. R. Petruzielo, A. A. Holmes, H. J. Changlani, M. P. Nightingale, and C. J. Umrigar, Phys. Rev. Lett. 109, 230201 (2012).

[55] G. H. Booth and G. K.-L. Chan, J. Chem. Phys. 137, 191102 (2012).

[56] H. M. Senn and W. Thiel, Angew. Chem. Int. Ed. 48, 1198 (2009).

[57] A. D. Mackerell, J. Comput. Chem. 25, 1584 (2004).

[58] A. Laio, J. VandeVondele, and U. Rothlisberger, J. Chem. Phys. 116, 6941 (2002).

[59] A. Laio, J. VandeVondele, and U. Rothlisberger, J. Phys. Chem. B 106, 7300 (2002).

[60] CPMD, http://www.cpmd.org/, Copyright IBM Corp 1990-2008, Copyright MPI für Festkörperforschung Stuttgart 1997-2001.

[61] R. Car and M. Parrinello, Phys. Rev. Lett. 55, 2471 (1985).

[62] J. E. Sanhueza, O. Tapia, W. G. Laidlaw, and M. Trsic, J. Chem. Phys. 70, 3096 (1979).

[63] R. Cammi, S. Corni, B. Mennucci, and J. Tomasi, J. Chem. Phys. 122, 104513 (2005). 
[64] S. Corni, R. Cammi, B. Mennucci, and J. Tomasi, J. Chem. Phys. 123, 134512 (2005).

[65] L. Gagliardi, R. Lindh, and G. Karlström, The Journal of Chemical Physics 121, 4494 (2004).

[66] B. Hess, C. Kutzner, D. van der Spoel, and E. Lindahl, J. Chem. Theory Comput. 4, 435 (2008).

[67] W. F. van Gunsteren, S. R. Billeter, A. A. Eising, P. H. Hünenberger, P. Krüger, A. E. Mark, W. R. P. Scott, I. G. Tironi, Biomolecular Simulation: The GROMOS96 Manual and User Guide; Vdf Hochschulverlag AG an der ETH Zurich: Zurich, 1996.

[68] F. Aquilante, L. De Vico, N. Ferré, G. Ghigo, P.-Å. Malmqvist, P. Neogrády, T. B. Pedersen, M. Pitoňák, M. Reiher, B. O. Roos, L. Serrano-Andrés, M. Urban, V. Veryazov, and R. Lindh, J. Comput. Chem. 31, 224 (2010).

[69] M. J. Frisch, G. W. Trucks, H. B. Schlegel, G. E. Scuseria, M. A. Robb, J. R. Cheeseman, G. Scalmani, V. Barone, B. Mennucci, G. A. Petersson, H. Nakatsuji, M. Caricato, X. Li, H. P. Hratchian, A. F. Izmaylov, J. Bloino, G. Zheng, J. L. Sonnenberg, M. Hada, M. Ehara, K. Toyota, R. Fukuda, J. Hasegawa, M. Ishida, T. Nakajima, Y. Honda, O. Kitao, H. Nakai, T. Vreven, J. J. A. Montgomery, J. E. Peralta, F. Ogliaro, M. Bearpark, J. J. Heyd, E. Brothers, K. N. Kudin, V. N. Staroverov, R. Kobayashi, J. Normand, K. Raghavachari, A. Rendell, J. C. Burant, S. S. Iyengar, J. Tomasi, M. Cossi, N. Rega, J. M. Millam, M. Klene, J. E. Knox, J. B. Cross, V. Bakken, C. Adamo, J. Jaramillo, R. Gomperts, R. E. Stratmann, O. Yazyev, A. J. Austin, R. Cammi, C. Pomelli, J. W. Ochterski, R. L. Martin, K. Morokuma, V. G. Zakrzewski, G. A. Voth, P. Salvador, J. J. Dannenberg, S. Dapprich, A. D. Daniels, Ö. Farkas, J. B. Foresman, J. V. Ortiz, J. Cioslowski, and D. J. Fox, Gaussian 09 Revision A.02, Gaussian Inc. Wallingford CT 2009.

[70] ADF2012, SCM, Theoretical Chemistry, Vrije Universiteit, Amsterdam, The Netherlands, http://www.scm.com.

[71] M. W. Schmidt, K. K. Baldridge, J. A. Boatz, S. T. Elbert, M. S. Gordon, J. H. Jensen, S. Koseki, N. Matsunaga, K. A. Nguyen, S. Su, T. L. Windus, M. Dupuis, and J. A. M. Jr, J. Comput. Chem. 14, 1347 (1993).

[72] DALTON, an ab initio electronic structure program, release 2.0, see http://www.kjemi.uio.no/software/dalton/dalton.html (2005).

[73] R. Ahlrichs, M. Bär, M. Häser, H. Horn, and C. Kölmel, Chem. Phys. Lett. 162, 165 (1989).

[74] CFOUR, Coupled-Cluster techniques for Computational Chemistry, a quantum-chemical program package by J.F. Stanton, J. Gauss, M.E. Harding, 
P.G. Szalay with contributions from A.A. Auer, R.J. Bartlett, U. Benedikt, C. Berger, D.E. Bernholdt, Y.J. Bomble, L. Cheng, O. Christiansen, M. Heckert, O. Heun, C. Huber, T.-C. Jagau, D. Jonsson, J. Jusélius, K. Klein, W.J. Lauderdale, D.A. Matthews, T. Metzroth, L.A. Mück, D.P. O’Neill, D.R. Price, E. Prochnow, C. Puzzarini, K. Ruud, F. Schiffmann, W. Schwalbach, S. Stopkowicz, A. Tajti, J. Vázquez, F. Wang, J.D. Watts and the integral packages MOLECULE (J. Almlöf and P.R. Taylor), PROPS (P.R. Taylor), ABACUS (T. Helgaker, H.J. Aa. Jensen, P. Jørgensen, and J. Olsen), and ECP routines by A. V. Mitin and C. van Wüllen. For the current version, see http://www.cfour.de. (accessed July 12, 2012).

[75] Neese, F. ORCA - an ab initio, Density Functional and Semiempirical program package, Version 2.8. Max-Planck-Insitut für Bioanorganische Chemie, Mülheim an der Ruhr, 2011. 



\section{Chapter 3}

\section{State-Specific Embedding Potentials for Excitation-Energy Calculations $^{\dagger}$}

Embedding potentials are frequently used to describe the effect of an environment on the electronic structure of molecules in larger systems, including their excited states. If such excitations are accompanied by significant rearrangements in the electron density of the embedded molecule, large differential polarization effects may take place, which in turn can require state-specific embedding potentials for an accurate theoretical description. We outline here how to extend wave function in density functional theory (WF/DFT) methods to compute the excitation energies of a molecule in a responsive environment through the use of state-specific density-based embedding potentials constructed within a modified subsystem DFT approach. We evaluate the general expression of the ground- and excited-state energy difference of the total system both with the use of state-independent and state-dependent embedding potentials, and propose some practical recipes to construct the approximate excitedstate DFT density of the active part used to polarize the environment. We illustrate these concepts with the state-independent and state-dependent WF/DFT computation of the excitation energies of $p$-nitroaniline, acrolein, methylenecyclopropene, and $p$ nitrophenolate in various solvents.

\subsection{Introduction}

A well-known strategy for electronic structure calculations on complex systems is to partition the system into an active region (system 1) and an environment (system 2), and then to employ a hybrid energy expression. In so-called additive hybrid schemes, the energy expression is written as

$$
E_{(1+2)}^{\text {hybrid }}=E_{1}^{M_{A}}+E_{2}^{M_{B}}+E_{(1 \leftrightarrow 2)}^{M_{C}},
$$

\footnotetext{
$\dagger$ This chapter has been published as C. Daday, C. König, O. Valsson, J. Neugebauer, and C. Filippi, "State-Specific Embedding Potentials for Excitation-Energy Calculations", J. Chem. Theory Comput. 2013, 9, 2355-2367
} 
where $M_{A / B / C}$ denote the different theoretical methods used to calculate the energies of system 1 and 2, and the interaction energy $E_{(1 \leftrightarrow 2)}$. Often, methods $M_{B}$ and $M_{C}$ are identical. Several combinations of different methods are possible [1-5], with the class of mixed quantum mechanics in molecular mechanics (QM/MM) methods being probably the most prominent ones [1]. In these methods, a polarization of system 1 due to the environment is achieved by including an embedding potential, in the simplest case just due to a set of point charges. If also the polarization of the environment by the active system shall be described, it is necessary to employ polarizable force fields.

Extensions for electronic excitations are also known in combination with the two standard ways of calculating excitation energies in electronic-structure theory $[6,7]$, namely, directly through a linear-response approach (i.e. as poles of the dynamic polarizability of the system) or as the difference between energies of two distinct electronic states of the combined system $(1+2)$. Since the electron density of system 1 changes upon excitation, the mutual polarization between systems 1 and 2 will be different in the two electronic states. The resulting effect on excitation energies is known as the differential polarization. In response-based QM/MM methods, these effects can be described by including the response of the environment, for instance via induced dipole moments [8-10], while, in energy-differences based QM/MM methods, they can be easily obtained if polarizable MM models for the environment are employed: The polarization of the environment depends on the charge distribution of the embedded system, and changes in the charge distribution immediately lead to a change in the polarization. It should be emphasized that, in both theoretical frameworks, we aim at describing the combined or supersystem $(1+2)$, so we want to describe either the response of the entire supersystem or a particular excited state of the supersystem in addition to its ground state.

Sub-system density-functional theory (DFT) [11,12], though in principle an alternative formulation of DFT, can be regarded as a hybrid method of Kohn-Sham (KS) DFT for system 1 and 2 in which the interaction energy is calculated based on orbital-free (OF) DFT [13]. By minimizing the total energy expression with respect to the density of system $1\left(\rho_{1}\right)$ given a fixed density of the environment $\left(\rho_{2}\right)$, one derives a set of Kohn-Sham-like equations for the optimal $\rho_{1}$, in which an effective embedding potential arises due to the presence of $\rho_{2}$ [14]. Since the same can also be done for system 2, a scheme is derived in which systems 1 and 2 are iteratively polarized with respect to each other, until self-consistency [15]. In this way, subsystem DFT can be regarded as a fully self-consistent alternative to KS-DFT $[5,16]$. As far as excited-state calculations are concerned, the environmental response can be included in the corresponding response-based subsystem time-dependent density functional theory (TDDFT) [17-19].

Following the pioneering work by the Carter group [20,21], several authors have dealt with the question of how to derive and numerically develop, in a similar spirit, wave function/density functional theory (WF/DFT) hybrid methods [7, 22-28]. In these methods, density-based embedding potentials are used to include polarization effects [29] caused by the environment, while accurate correlated wave function methods can be employed for the active system. Both state-dependent [30-34] and 
response-based methods [35] have been suggested, which are in principle capable of capturing differential polarization. Although this issue has received significant attention in recent years, to the best of our knowledge, no numerical applications have been presented yet containing these contributions.

Here, we investigate possible routes for extending hybrid WF/DFT approaches for the calculation of excited states. We do not consider response-based approaches, but concentrate on state-dependent methods. We will analyze different types of approximations that can or have to be made to make WF/DFT methods applicable in practice. We will compare the use of effective "embedding operators" in the calculation of the energy of system 1 with the explicit evaluation of the entire energydifference expression. These approaches will be analyzed for state-independent (no differential polarization) and state-specific (differential polarization included) embedding potentials.

We will start with a presentation of the theory in Section 3.2, followed by details of the implementation and computations in Section 3.3. Results for a number of test cases are given in Section 3.4 before we summarize our conclusions in Section 3.5.

\subsection{Theory}

\subsubsection{DFT/DFT and WF/DFT embedding methods}

We start by writing the subsystem DFT energy of a supersystem composed of two subsystems (1 and 2) as a DFT/DFT hybrid energy expression,

$$
E_{(1+2)}^{\mathrm{DFT} / \mathrm{DFT}}=E_{1}^{\mathrm{KS}}\left[\rho_{1}\right]+E_{2}^{\mathrm{KS}}\left[\rho_{2}\right]+E_{(1 \leftrightarrow 2)}^{\mathrm{OFDFT}}\left[\rho_{1}, \rho_{2}\right] .
$$

The KS-DFT energies of system 1 and 2 in this expression are,

$$
E_{I}^{\mathrm{KS}}\left[\rho_{I}\right]=T_{s}\left[\left\{\phi_{i_{I}}\right\}\right]+J\left[\rho_{I}\right]+V_{\mathrm{nuc}, I}\left[\rho_{I}\right]+E_{x c}\left[\rho_{I}\right]
$$

where $I$ denotes system 1 or $2, J$ is the Coulomb energy of a given electron density distribution, $E_{x c}$ is the exchange-correlation energy, $V_{\text {nuc }, I}\left[\rho_{I}\right]$ is the interaction of the electron density of system $I$ with the nuclei in the same system, and $T_{s}\left[\left\{\phi_{i_{I}}\right\}\right]$ is the kinetic energy as defined in the Kohn-Sham formalism, calculated from the Kohn-Sham-like orbitals, $\left\{\phi_{i_{I}}\right\}$. The interaction energy is then,

$$
\begin{aligned}
E_{(1 \leftrightarrow 2)}^{\mathrm{OFDFT}}\left[\rho_{1}, \rho_{2}\right] & =V_{\text {nuc }, 1}\left[\rho_{2}\right]+V_{\text {nuc }, 2}\left[\rho_{1}\right]+J\left[\rho_{1}, \rho_{2}\right] \\
& +E_{x c}^{\text {nadd }}\left[\rho_{1}, \rho_{2}\right]+T_{s}^{\text {nadd }}\left[\rho_{1}, \rho_{2}\right],
\end{aligned}
$$

where $V_{\text {nuc }, I}\left[\rho_{K}\right]$ is the Coulomb attraction between the nuclei of system $I$ and the electron density of system $K, J\left[\rho_{1}, \rho_{2}\right]$ is the Coulomb repulsion between the electron densities of the two systems,

$$
J\left[\rho_{1}, \rho_{2}\right]=\iint \frac{\rho_{1}\left(\mathbf{r}_{1}\right) \rho_{2}\left(\mathbf{r}_{2}\right)}{\left|\mathbf{r}_{1}-\mathbf{r}_{2}\right|} \mathrm{d} \mathbf{r}_{1} \mathrm{~d} \mathbf{r}_{2}
$$


and the non-additive density functionals $F^{\text {nadd }}=\left\{E_{x c}^{\text {nadd }}, T_{s}^{\text {nadd }}\right\}$ are defined as,

$$
F^{\text {nadd }}\left[\rho_{1}, \rho_{2}\right]=F\left[\rho_{1}+\rho_{2}\right]-F\left[\rho_{1}\right]-F\left[\rho_{2}\right] .
$$

By minimizing Eq. (3.2) with respect to the density $\rho_{1}$ (keeping $\rho_{2}$ fixed), one obtains a set of Kohn-Sham-like equations containing an effective embedding potential [14],

$$
\begin{aligned}
v_{\mathrm{emb}, 1}(\mathbf{r})= & v_{\text {nuc }, 2}(\mathbf{r})+\int \frac{\rho_{2}\left(\mathbf{r}^{\prime}\right)}{\left|\mathbf{r}-\mathbf{r}^{\prime}\right|} \mathrm{d} \mathbf{r}^{\prime} \\
& +\frac{\delta E_{x c}^{\text {nadd }}\left[\rho_{1}, \rho_{2}\right]}{\delta \rho_{1}(\mathbf{r})}+\frac{\delta T^{\text {nadd }}\left[\rho_{1}, \rho_{2}\right]}{\delta \rho_{1}(\mathbf{r})} .
\end{aligned}
$$

Here, $v_{\text {nuc }, 2}$ is the Coulomb potential due to the nuclei of system 2. A similar embedding potential, $v_{\mathrm{emb}, 2}(\mathbf{r})$ (with indices 1 and 2 interchanged in $v_{\mathrm{emb}, 1}$ ) is obtained by minimizing the energy with respect to $\rho_{2}$. In practical applications, an iterative "freeze-and-thaw" scheme can be adopted in which systems 1 and 2 are mutually polarized until self-consistency [15].

If the energy of system 1 shall be described in terms of a wave function method, we can, according to Eq. (3.1), employ the following WF/DFT energy expression,

$$
E_{(1+2)}^{\mathrm{WF} / \mathrm{DFT}}=E_{1}^{\mathrm{WF}}\left[\Psi_{1}\right]+E_{2}^{\mathrm{KS}}\left[\rho_{2}\right]+E_{(1 \leftrightarrow 2)}^{\mathrm{OFDFT}}\left[\rho_{1}, \rho_{2}\right]
$$

with

$$
E_{1}^{\mathrm{WF}}\left[\Psi_{1}\right]=\left\langle\Psi_{1}\left|\left\{\sum_{i}\left[-\frac{1}{2} \nabla_{i}^{2}+v_{\mathrm{nuc}, 1}\left(\mathbf{r}_{i}\right)\right]+\sum_{i<j} \frac{1}{r_{i j}}\right\}\right| \Psi_{1}\right\rangle,
$$

where the sum is over the electrons of system 1 . In the WF/DFT context, the wave function $\Psi_{1}$ is obtained under the influence of the embedding potential $v_{\mathrm{emb}, 1}(\mathbf{r})$, i.e. with a modified one-electron operator [36],

$$
\left\{\sum_{i}\left[-\frac{1}{2} \nabla_{i}^{2}+v_{\text {nuc }, 1}\left(\mathbf{r}_{i}\right)+v_{\mathrm{emb}, 1}\left(\mathbf{r}_{i}\right)\right]+\sum_{i<j} \frac{1}{r_{i j}}\right\} \Psi_{1}=\tilde{E}_{1}^{\mathrm{WF}} \Psi_{1} .
$$

A similar embedding potential $\left(v_{\mathrm{emb}, 2}\right)$ has to be included in the determination of $\rho_{2}$ within the KS-like equations for system 2. This potential depends on the density of system 1, so an iterative polarization of active part and environment needs to be carried out, similar to the freeze-and-thaw scheme in the DFT/DFT context. Since this is usually very time-consuming and technically difficult with highly correlated WF methods, one can resort to approximate schemes in which the freeze-and-thaw cycles are carried out in a DFT/DFT embedding framework, and the resulting embedding potential is taken as an approximation for the fully self-consistent WF/DFT embedding potential [37]. As an alternative, Carter and co-workers have employed an environmental density $\rho_{2}$ which was calculated from a DFT-based density of the total system, from which the density of the (isolated) system 1 was subtracted [23]. 


\subsubsection{Excitation energies in WF/DFT}

As mentioned before, here we will only consider state-dependent electronic-structure methods, and thus not discuss response-based treatments. Excitation energies $\Delta E$ are therefore calculated explicitly as energy differences between excited and ground electronic states. In the framework of WF/DFT embedding, this means,

$$
\Delta E=E_{(1+2) e}^{\mathrm{WF} / \mathrm{DFT}}-E_{(1+2) g}^{\mathrm{WF} / \mathrm{DFT}}
$$

where the subscripts $e$ and $g$ refer to the excited and the ground state, respectively. The main question in this work is how to approximate the calculation of this energy difference, and how different approximations affect the resulting excitation energies.

It has also been argued $[7,32,35]$ and is physically reasonable that state-specific embedding potentials are needed for a consistent theoretical description of excitation energies in WF/DFT, which include differential polarization effects. In particular, Khait and Hoffmann pointed out that WF/DFT embedding is formally correct not only for ground but also for excited states [32]. They make use of the observation by Perdew and Levy in the context of pure DFT [38] that every extremum of the energy functional corresponds to a stationary-state density. Since, however, not every stationary-state density is a also an extremum of the energy functional, the formal correctness only holds for a subset of excited states. In spite of this difficulty, we are going to investigate how a practical scheme for state-specific embedding potentials in WF/DFT calculations can be implemented, and whether the increased effort is justified by the results.

We will first neglect differential polarization effects. If the excitation is entirely localized within the embedded system (system 1), it is often a good approximation to assume that system 2 does not respond to the excitation of system 1 so that the density $\rho_{2}$ is the same in both calculations. The embedding potentials for ground- and excited-state calculations derived from $\rho_{2}$ will also be very similar since the (often dominant) electrostatic contribution is unchanged. However, they will not be precisely the same since the non-additive parts of the orbital-free embedding potential depend also on $\rho_{1}$, which is different for the two states. In addition, one can further approximate the DFT-based embedding potential by calculating it from a fixed guess for the density of the active system, [39] a strategy that has been used in the context of WF/DFT embedding before [37]. This has the advantage that the embedding potential can be generated on a grid, and then be re-used in both the ground- and excited-state calculations.

Writing the energy difference explicitly with the assumption of no differential polarization ( $\rho_{2}$ unchanged), we obtain

$$
\begin{aligned}
\Delta E^{\mathrm{no}-\text { dpol }} & =\left(E_{1}^{\mathrm{WF}}\left[\Psi_{1}^{e}\right]+E_{2}^{\mathrm{KS}}\left[\rho_{2}\right]+E_{(1 \leftrightarrow 2)}^{\mathrm{OFDFT}}\left[\rho_{1}^{e}, \rho_{2}\right]\right) \\
& -\left(E_{1}^{\mathrm{WF}}\left[\Psi_{1}^{g}\right]+E_{2}^{\mathrm{KS}}\left[\rho_{2}\right] E_{(1 \leftrightarrow 2)}^{\mathrm{OFDT}}\left[\rho_{1}^{g}, \rho_{2}\right]\right) \\
& =E_{1}^{\mathrm{WF}}\left[\Psi_{1}^{e}\right]+E_{(1 \leftrightarrow 2)}^{\mathrm{OFDFT}}\left[\rho_{1}^{e}, \rho_{2}\right] \\
& -\left(E_{1}^{\mathrm{WF}}\left[\Psi_{1}^{g}\right]+E_{(1 \leftrightarrow 2)}^{\mathrm{OFDFT}}\left[\rho_{1}^{g}, \rho_{2}\right]\right)
\end{aligned}
$$


where we denote the ground- and excited-state wave functions with $\Psi_{1}^{g}$ and $\Psi_{1}^{e}$, and the corresponding densities with $\rho_{1}^{g}$ and $\rho_{1}^{e}$. Note that, in addition to $E_{2}^{\mathrm{KS}}\left[\rho_{2}\right]$, the term $V_{\text {nuc }, 1}\left[\rho_{2}\right]$ contained in $E_{(1 \leftrightarrow 2)}^{\mathrm{OFDFT}}$ will also cancel out in the above expression.

If we considered only electrostatic interaction terms, i.e. neglected all differential non-additive terms, then the excitation energy would be:

$$
\begin{aligned}
\Delta E^{\mathrm{elstat}} & =\left(E_{1}^{\mathrm{WF}}\left[\Psi_{1}^{e}\right]-E_{1}^{\mathrm{WF}}\left[\Psi_{1}^{g}\right]\right)+V_{\mathrm{nuc}, 2}\left[\rho_{1}^{e}\right]-V_{\mathrm{nuc}, 2}\left[\rho_{1}^{g}\right] \\
& +J\left[\rho_{1}^{e}, \rho_{2}\right]-J\left[\rho_{1}^{g}, \rho_{2}\right] \\
& =\left(E_{1}^{\mathrm{WF}}\left[\Psi_{1}^{e}\right]-E_{1}^{\mathrm{WF}}\left[\Psi_{1}^{g}\right]\right) \\
& +\left\langle\Psi_{1}^{e}\left|\sum_{i} v_{\mathrm{emb}, 1}^{\text {elstat }}\left(\mathbf{r}_{i}\right)\right| \Psi_{1}^{e}\right\rangle-\left\langle\Psi_{1}^{g}\left|\sum_{i} v_{\mathrm{emb}, 1}^{\text {elstat }}\left(\mathbf{r}_{i}\right)\right| \Psi_{1}^{g}\right\rangle,
\end{aligned}
$$

where we introduced the electrostatic component of the embedding potential,

$$
v_{\mathrm{emb}, 1}^{\mathrm{elstat}}(\mathbf{r})=v_{\text {nuc }, 2}(\mathbf{r})+\int \frac{\rho_{2}\left(\mathbf{r}^{\prime}\right)}{\left|\mathbf{r}-\mathbf{r}^{\prime}\right|} \mathrm{d} \mathbf{r}^{\prime} .
$$

Evaluating excitation energies according to Eq. (3.13) would be particularly simple since it corresponds to computing the differences of the modified energies $\tilde{E}_{1}^{\mathrm{WF}}$ from Eq. (3.10) (only considering the electrostatic contribution in the embedding potential).

It is thus appealing to compute approximate excitation energies from WF/DFT as a simple difference in the ground- and excited-state eigenvalues of a modified Hamiltonian including the (ground-state-like) embedding potential as in Eq. (3.10), now containing all contributions,

$$
\begin{aligned}
\Delta E_{\text {approx }}^{(A)} & =E_{1}^{\mathrm{WF}}\left[\Psi_{1}^{e}\right]+\left\langle\Psi_{1}^{e}\left|\sum_{i} v_{\mathrm{emb}, 1}\left(\mathbf{r}_{i}\right)\right| \Psi_{1}^{e}\right\rangle \\
& -\left(E_{1}^{\mathrm{WF}}\left[\Psi_{1}^{g}\right]+\left\langle\Psi_{1}^{g}\left|\sum_{i} v_{\mathrm{emb}, 1}\left(\mathbf{r}_{i}\right)\right| \Psi_{1}^{g}\right\rangle\right) \\
& =E_{1}^{\mathrm{WF}}\left[\Psi_{1}^{e}\right]-E_{1}^{\mathrm{WF}}\left[\Psi_{1}^{g}\right]+V_{\text {nuc }, 2}\left[\rho_{1}^{e}\right]-V_{\text {nuc }, 2}\left[\rho_{1}^{g}\right] \\
& +J\left[\rho_{1}^{e}, \rho_{2}\right]-J\left[\rho_{1}^{g}, \rho_{2}\right] \\
& +\int v_{x c}^{\text {nadd }}\left[\rho_{1}^{g}, \rho_{2}\right](\mathbf{r})\left[\rho_{1}^{e}(\mathbf{r})-\rho_{1}^{g}(\mathbf{r})\right] \mathrm{d} \mathbf{r} \\
& +\int v_{t}^{\text {nadd }}\left[\rho_{1}^{g}, \rho_{2}\right](\mathbf{r})\left[\rho_{1}^{e}(\mathbf{r})-\rho_{1}^{g}(\mathbf{r})\right] \mathrm{d} \mathbf{r},
\end{aligned}
$$

where we denote the ground- and excited-state wave functions in the potential $v_{\mathrm{emb}, 1}$ [Eq. (3.10)] with $\Psi_{1}^{g}$ and $\Psi_{1}^{e}$, and the corresponding densities with and $\rho_{1}^{g}$ and $\rho_{1}^{e}$. The potentials $v_{x c}^{\text {nadd }}$ and $v_{t}^{\text {nadd }}$ are the functional derivatives of the corresponding energy functionals as appearing in Eq. (3.7). The use of a ground-state-like embedding potential in the ground- and excited-state WF calculations will be denoted as route (A) in the following. It is equivalent to the "first-order corrected" approach employed by Carter and co-workers in Ref. [34], while they used the approximation

$$
\Delta E^{\mathrm{no}-\mathrm{dpol}} \approx E_{1}^{\mathrm{WF}}\left[\Psi_{1}^{e}\right]-E_{1}^{\mathrm{WF}}\left[\Psi_{1}^{g}\right],
$$


in their earlier work $[30,31,33]$. This older approximation does however not contain the change in the electrostatic interaction contributions between the subsystems, which has been shown to substantially affect the excitation energies of $\mathrm{Mg}_{n} \mathrm{O}_{n}$ clusters [34].

\subsubsection{State-specific vs. state-independent embedding}

A second route in our current work - denoted with (B) - is to allow system 2 to respond to the excitation of 1 , and in turn to include "back" polarization effects on system 1 . This is equivalent to saying that the energy difference contains differential polarization effects, which requires state-specific embedding potentials.

We rewrite the general expression of the energy of the total system in an excited state localized on system 1 as

$$
E_{(1+2) e}^{\mathrm{WF} / \mathrm{DFT}}=E_{1}^{\mathrm{WF}}\left[\tilde{\Psi}_{1}^{e}\right]+E_{2}^{\mathrm{KS}}\left[\tilde{\rho}_{2}\right]+E_{(1 \leftrightarrow 2)}^{\mathrm{OFDFT}}\left[\tilde{\rho}_{1}^{e}, \tilde{\rho}_{2}\right]
$$

where $\tilde{\Psi}_{1}^{e}$ denotes the excited-state wave function computed in an embedding potential which accounts for differential polarization. In principle, all energy expressions for ground and excited states have to be evaluated with self-consistently optimized subsystem densities/wave functions [32] so that also the energy of system 2 and the interaction energy in the above expression differ from the ones in the groundstate case, Eq. (3.8). We denote these general, self-consistent quantities entering in Eq. (3.22) with a tilde, and discuss below some specific, practical choices for their computation.

The general excitation energy is given by the difference between this WF/DFT excited-state energy and the ground-state one of Eq. (3.8):

$$
\begin{aligned}
\Delta E & =E_{(1+2) e}^{\mathrm{WF} / \mathrm{DFT}}-E_{(1+2) g}^{\mathrm{WF} / \mathrm{DFT}} \\
& =E_{1}^{\mathrm{WF}}\left[\tilde{\Psi}_{1}^{e}\right]-E_{1}^{\mathrm{WF}}\left[\Psi_{1}^{g}\right]+E_{2}^{\mathrm{KS}}\left[\tilde{\rho}_{2}\right]-E_{2}^{\mathrm{KS}}\left[\rho_{2}\right] \\
& +E_{(1 \leftrightarrow 2)}^{\mathrm{OFDFT}}\left[\tilde{\rho}_{1}^{e}, \tilde{\rho}_{2}\right]-E_{(1 \leftrightarrow 2)}^{\mathrm{OFDFT}}\left[\rho_{1}^{g}, \rho_{2}\right] \\
& =E_{1}^{\mathrm{WF}}\left[\tilde{\Psi}_{1}^{e}\right]-E_{1}^{\mathrm{WF}}\left[\Psi_{1}^{g}\right]+E_{2}^{\mathrm{KS}}\left[\tilde{\rho}_{2}\right]-E_{2}^{\mathrm{KS}}\left[\rho_{2}\right] \\
& +V_{\mathrm{nuc}, 1}\left[\tilde{\rho}_{2}\right]-V_{\mathrm{nuc}, 1}\left[\rho_{2}\right]+V_{\text {nuc }, 2}\left[\tilde{\rho}_{1}^{e}\right]-V_{\text {nuc }, 2}\left[\rho_{1}^{g}\right]+J\left[\tilde{\rho}_{1}^{e}, \tilde{\rho}_{2}\right]-J\left[\rho_{1}^{g}, \rho_{2}\right] \\
& +E_{x c}^{\mathrm{nadd}}\left[\tilde{\rho}_{1}^{e}, \tilde{\rho}_{2}\right]-E_{x c}^{\mathrm{nadd}}\left[\rho_{1}^{g}, \rho_{2}\right]+T_{s}^{\text {nadd }}\left[\tilde{\rho}_{1}^{e}, \tilde{\rho}_{2}\right]-T_{s}^{\mathrm{nadd}}\left[\rho_{1}^{g}, \rho_{2}\right]
\end{aligned}
$$

where, for clarity, we denote the ground-state solutions for system 1 in Eq. (3.8) as $\Psi_{1}^{g}$ and $\rho_{1}^{g}$.

For both cases (A) and (B), the practical implementation of the ground-state energy calculations is the same: Following for instance Ref. [37], we do not employ the WF density of system 1 but perform an iterative "freeze-and-thaw" scheme within subsystem DFT, in which systems 1 and 2 are mutually polarized in the ground state until self-consistency. We then construct an embedding potential $v_{\mathrm{emb}, 1}(\mathbf{r})$ based on the final ground-state DFT densities of systems 1 and 2 to obtain the embedded ground-state wave function $\Psi_{1}^{g}$ of system 1 . 
For the excited-state calculation in case (A), the ground-state-like embedding potential $v_{\text {emb, } 1}(\mathbf{r})$ is also used to calculate an excited-state wave function, $\tilde{\Psi}_{1}^{e}=\Psi_{1}^{e}$, and the corresponding density $\tilde{\rho}_{1}^{e}=\rho_{1}^{e}$. The density of system 2 remains unchanged so that $\tilde{\rho}_{2}=\rho_{2}$. The excitation energy in case (A) can be calculated as:

$$
\begin{aligned}
\Delta E^{(A)} & =E_{1}^{\mathrm{WF}}\left[\Psi_{1}^{e}\right]-E_{1}^{\mathrm{WF}}\left[\Psi_{1}^{g}\right]+V_{\text {nuc }, 2}\left[\rho_{1}^{e}\right]-V_{\text {nuc }, 2}\left[\rho_{1}^{g}\right] \\
& +J\left[\rho_{1}^{e}, \rho_{2}\right]-J\left[\rho_{1}^{g}, \rho_{2}\right]+E_{x c}^{\text {nadd }}\left[\rho_{1}^{e}, \rho_{2}\right]-E_{x c}^{\text {nadd }}\left[\rho_{1}^{g}, \rho_{2}\right] \\
& +T_{s}^{\text {nadd }}\left[\rho_{1}^{e}, \rho_{2}\right]-T_{s}^{\text {nadd }}\left[\rho_{1}^{g}, \rho_{2}\right],
\end{aligned}
$$

where the $E_{2}^{\mathrm{KS}}\left[\rho_{2}\right]$ and $V_{\text {nuc, },}\left[\rho_{2}\right]$ terms cancel in the difference of the interaction energies. If we compare $\Delta E^{(A)}$ with the approximate expression (Eq. 3.17), we observe that the electrostatic interaction terms, which often dominate environment effects on excitation energies, are treated exactly in $\Delta E_{\text {approx }}^{(A)}$ (for the given densities), and that the approximation in Eq. (3.17) amounts to neglecting the following correction term,

$$
\begin{aligned}
\delta E_{\text {nadd }}^{(\mathrm{A})} & =\Delta E^{(A)}-\Delta E_{\text {approx }}^{(A)} \\
& =E_{x c}^{\text {nadd }}\left[\rho_{1}^{e}, \rho_{2}\right]-E_{x c}^{\text {nadd }}\left[\rho_{1}^{g}, \rho_{2}\right]+T_{s}^{\text {nadd }}\left[\rho_{1}^{e}, \rho_{2}\right]-T_{s}^{\text {nadd }}\left[\rho_{1}^{g}, \rho_{2}\right] \\
& -\int\left\{v_{x c}^{\text {nadd }}\left[\rho_{1}^{g}, \rho_{2}\right](\mathbf{r})+v_{t}^{\text {nadd }}\left[\rho_{1}^{g}, \rho_{2}\right](\mathbf{r})\right\}\left[\rho_{1}^{e}(\mathbf{r})-\rho_{1}^{g}(\mathbf{r})\right] \mathrm{d} \mathbf{r} .
\end{aligned}
$$

Although there is no rigorous justification for this approximation, we observe that (i) all ingredients here are "non-additive" terms, which go to zero for non-interacting systems and will be small for weakly interacting systems, (ii) we are taking differences between these terms evaluated with very similar (or even equal, for $\rho_{2}$ ) densities, and (iii) exchange-correlation and kinetic-energy contributions often cancel out to a certain degree. Therefore, this approximation can be expected to be rather good in practical applications.

For the excited-state calculation in case (B), where the WF/DFT hybrid scheme is improved by considering differential polarization effects, we construct a new embedding potential $v_{\mathrm{emb}, 2}^{\text {pol }}(\mathbf{r})$ based on an approximate excited-state density of system 1. With this potential, we calculate a ground-state density $\tilde{\rho}_{2}=\rho_{2}^{p o l}(\mathbf{r})$ that is polarized with respect to the excited-state density of system 1 . Then, we use $\rho_{2}^{p o l}(\mathbf{r})$ to construct an excited-state-like embedding potential $v_{\mathrm{emb}, 1}^{\text {pol,e }}(\mathbf{r})$, and then iterate until self-consistency. The final $v_{\mathrm{emb}, 1}^{\text {pol, }}(\mathbf{r})$ is employed to calculate an excited-state wave function, $\tilde{\Psi}_{1}^{e}=\Psi_{1}^{p o l, e}$, and corresponding density $\tilde{\rho}_{1}^{e}=\rho_{1}^{p o l, e}(\mathbf{r})$. The full excitationenergy expression is:

$$
\begin{aligned}
\Delta E^{(B)} & =E_{1}^{\mathrm{WF}}\left[\Psi_{1}^{\text {pol }, e}\right]-E_{1}^{\mathrm{WF}}\left[\Psi_{1}^{g}\right]+E_{2}^{\mathrm{KS}}\left[\rho_{2}^{\text {pol }}\right]-E_{2}^{\mathrm{KS}}\left[\rho_{2}\right] \\
& +V_{\text {nuc }, 1}\left[\rho_{2}^{\text {pol }}\right]-V_{\text {nuc }, 1}\left[\rho_{2}\right]+V_{\text {nuc }, 2}\left[\rho_{1}^{\text {pol,ee}}\right]-V_{\text {nuc }, 2}\left[\rho_{1}^{g}\right] \\
& +J\left[\rho_{1}^{\text {pol }, e}, \rho_{2}^{\text {pol }}\right]-J\left[\rho_{1}^{g}, \rho_{2}\right]+E_{x c}^{\text {nadd }}\left[\rho_{1}^{\text {pol }, e}, \rho_{2}^{\text {pol }}\right]-E_{x c}^{\text {nadd }}\left[\rho_{1}^{g}, \rho_{2}\right] \\
& +T_{s}^{\text {nadd }}\left[\rho_{1}^{\text {pol }, e}, \rho_{2}^{\text {pol }}\right]-T_{s}^{\text {nadd }}\left[\rho_{1}^{g}, \rho_{2}\right]
\end{aligned}
$$

where the situation changes with respect to case (A) as the energy contributions for system 2 do not cancel anymore. 
In analogy to case (A), the approximate form of the excitation energy where the system-interaction is accounted for via expectation values of embedding potentials is given by

$$
\begin{aligned}
& \Delta E_{\mathrm{approx}}^{(B)}=E_{1}^{\mathrm{WF}}\left[\Psi_{1}^{p o l, e}\right]+\left\langle\Psi_{1}^{\text {pol,e }}\left|\sum_{i} v_{\mathrm{emb}, 1}^{\text {pol,e }}\left(\mathbf{r}_{i}\right)\right| \Psi_{1}^{\text {pol,ee }}\right\rangle \\
& -\left(E_{1}^{\mathrm{WF}}\left[\Psi_{1}^{g}\right]+\left\langle\Psi_{1}^{g}\left|\sum_{i} v_{\mathrm{emb}, 1}\left(\mathbf{r}_{i}\right)\right| \Psi_{1}^{g}\right\rangle\right) \\
& =E_{1}^{\mathrm{WF}}\left[\Psi_{1}^{p o l, e}\right]-E_{1}^{\mathrm{WF}}\left[\Psi_{1}^{g}\right]+V_{\mathrm{nuc}, 2}\left[\rho_{1}^{p o l, e}\right]-V_{\text {nuc }, 2}\left[\rho_{1}^{g}\right] \\
& +\quad J\left[\rho_{1}^{p o l, e}, \rho_{2}^{\text {pol }}\right]-J\left[\rho_{1}^{g}, \rho_{2}\right] \\
& +\int\left[v_{x c}^{\text {nadd }}\left[\rho_{1}^{p o l, e}, \rho_{2}^{p o l}\right](\mathbf{r})+v_{t}^{\text {nadd }}\left[\rho_{1}^{p o l, e}, \rho_{2}^{p o l}\right](\mathbf{r})\right] \rho_{1}^{p o l, e}(\mathbf{r}) \mathrm{d} \mathbf{r} \\
& -\int\left[v_{x c}^{\text {nadd }}\left[\rho_{1}^{g}, \rho_{2}\right](\mathbf{r})+v_{t}^{\text {nadd }}\left[\rho_{1}^{g}, \rho_{2}\right](\mathbf{r})\right] \rho_{1}^{g}(\mathbf{r}) \mathrm{d} \mathbf{r} .
\end{aligned}
$$

Even if we assume that we can disregard all differential kinetic-energy and exchangecorrelation terms appearing as corrections to $E_{1}^{\mathrm{WF}}\left[\Psi_{1}^{p o l, e}\right]-E_{1}^{\mathrm{WF}}\left[\Psi_{1}^{g}\right]$, we nevertheless make the following approximation compared to the full excited-state expression [Eq. (3.26)]:

$$
E_{2}^{\mathrm{KS}}\left[\rho_{2}^{p o l}\right]-E_{2}^{\mathrm{KS}}\left[\rho_{2}\right]+V_{\text {nuc }, 1}\left[\rho_{2}^{p o l}\right]-V_{\text {nuc }, 1}\left[\rho_{2}\right] \approx 0,
$$

which is, as far as the electrostatic terms are concerned, equivalent to the following approximation,

$$
\int\left\{v_{\text {Coul }}\left[\rho_{2}+\Delta \rho_{2} / 2\right](\mathbf{r})+v_{\text {nuc }, 1}(\mathbf{r})+v_{\text {nuc }, 2}(\mathbf{r})\right\} \Delta \rho_{2}(\mathbf{r}) \mathrm{d} \mathbf{r} \approx 0,
$$

where $\Delta \rho_{2}(\mathbf{r})=\rho_{2}^{\text {pol }}(\mathbf{r})-\rho_{2}(\mathbf{r})$. This may be a rather drastic approximation, leading to errors of the same order of magnitude as the environment-induced shift that shall be calculated.

Our analysis reveals however a simple, practical cure to account for differential polarization effects and to remain within an approximation of the style of Eq. (3.27). We stress that the use of such an expression is, from a pragmatic point of view, preferable over the computation of the true excitation energy [Eq. (3.26)] since the one-electron integrals in the WF calculation can be used both in the variational optimization of the wave function and in the energy calculation. The use of Eq. (3.26) requires instead first to optimize the wave functions with the corresponding embedding potential and then to evaluate the energy expression without it. Fortunately, a simple solution is possible whose ingredients are to (i) calculate the change in the energy in system 2 explicitly and (ii) correct for the remaining Coulomb error, which leads to the simple correction

$$
\delta E_{\text {simple }}^{(B)}=E_{2}^{\mathrm{KS}}\left[\rho_{2}^{p o l}\right]-E_{2}^{\mathrm{KS}}\left[\rho_{2}\right]+\int v_{\text {nuc }, 1}(\mathbf{r}) \Delta \rho_{2}(\mathbf{r}) \mathrm{d} \mathbf{r} .
$$


An improved excitation energy with state-specific embedding potentials can then be obtained as

$$
\begin{aligned}
\Delta E_{\text {simple }}^{(B)} & =\Delta E_{\text {approx }}^{(B)}+\delta E_{\text {simple }}^{(B)} \\
& =E_{1}^{\mathrm{WF}}\left[\Psi_{1}^{p o l, e}\right]+\left\langle\Psi_{1}^{p o l, e}\left|\sum_{i} v_{\mathrm{emb}, 1}^{\text {pol,e }}\left(\mathbf{r}_{i}\right)\right| \Psi_{1}^{p o l, e}\right\rangle \\
& -E_{1}^{\mathrm{WF}}\left[\Psi_{1}^{g}\right]-\left\langle\Psi_{1}^{g}\left|\sum_{i} v_{\mathrm{emb}, 1}\left(\mathbf{r}_{i}\right)\right| \Psi_{1}^{g}\right\rangle \\
& +E_{2}^{\mathrm{KS}}\left[\rho_{2}^{\text {pol }}\right]-E_{2}^{\mathrm{KS}}\left[\rho_{2}\right]+\int v_{\mathrm{nuc}, 1}(\mathbf{r}) \Delta \rho_{2}(\mathbf{r}) \mathrm{d} \mathbf{r} .
\end{aligned}
$$

The remaining approximations are consistent with the ones made in Eq. (3.17) for a state-independent embedding potential and, to assess their impact, we can explicitly calculate the neglected terms,

$$
\begin{aligned}
\delta E_{\text {nadd }}^{(\mathrm{B})} & =E_{x c}^{\text {nadd }}\left[\rho_{1}^{\text {pol }, e}, \rho_{2}^{\text {pol }}\right]-E_{x c}^{\text {nadd }}\left[\rho_{1}^{g}, \rho_{2}\right] \\
& +T_{s}^{\text {nadd }}\left[\rho_{1}^{\text {pol,e }}, \rho_{2}^{\text {pol }}\right]-T_{s}^{\text {nadd }}\left[\rho_{1}^{g}, \rho_{2}\right] \\
& -\int\left[v_{x c}^{\text {nadd }}\left[\rho_{1}^{\text {pol }, e}, \rho_{2}^{\text {pol }}\right](\mathbf{r})+v_{t}^{\text {nadd }}\left[\rho_{1}^{\text {pol }, e}, \rho_{2}^{p o l}\right](\mathbf{r})\right] \rho_{1}^{\text {pol }, e}(\mathbf{r}) \mathrm{d} \mathbf{r} \\
& +\int\left[v_{x c}^{\text {nadd }}\left[\rho_{1}^{g}, \rho_{2}\right](\mathbf{r})+v_{t}^{\text {nadd }}\left[\rho_{1}^{g}, \rho_{2}\right](\mathbf{r})\right] \rho_{1}^{g}(\mathbf{r}) \mathrm{d} \mathbf{r}
\end{aligned}
$$

As mentioned above, in both schemes (A) and (B), we will introduce an additional approximation in practice and resort to the use of subsystem DFT densities for systems 1 and 2 to compute the embedding potentials needed in the WF/DFT calculations. For the ground-state calculations in both schemes, we follow the practical route to self-consistently converge the ground-state subsystem DFT densities of both systems, which are then used to construct the embedding potential, $v_{\mathrm{emb}, 1}(\mathbf{r})$, for the ground-state WF calculation of system 1. Similarly, for the excited-state calculation of scheme (B), we construct the excited-state-like embedding potential, $v_{\mathrm{emb}, 1}^{\mathrm{pol}, e}(\mathbf{r})$, from an approximate excited-state embedded DFT density of system 1 and the polarized ground-state DFT density of system 2, which are obtained self-consistently. In Section 4.1, we discuss two simple possible choices for approximate embedded excited-state DFT densities.

\subsection{Computational details}

To optimize the structures, we use either the Gaussian 09 [40] or the Turbomole code, version 6.0 [41]. We employ density functional theory (DFT) and the cc-pVDZ [42] basis set in combination with the B3LYP $[43,44]$ functional for $p$-nitroaniline and the BLYP $[45,46]$ functional for all other molecules.

All subsystem DFT calculations are performed using the ADF package [47]. We employ the M06-HF [48] exchange-correlation functional and the DZP [49] basis set. For the frozen-density embedding calculations, we use the PW91k kinetic-energy 
functional [50] to compute the non-additive terms of the kinetic energy since this functional has been shown to give a good performance in frozen density embedding [51]. For the non-additive exchange-correlation contribution, we employ the local density approximation (LDA) [52] since, in ADF, we cannot use the non-local M06-HF functional for this term as done in the intra-subsystem calculations. We have tested the use of PW91, instead of LDA, for all four molecules and found that the excitation energies within scheme (A) vary by less than $0.05 \mathrm{eV}$.

We perform the complete-active-space self-consistent field (CASSCF) and second-order perturbation theory (CASPT2) calculations using MOLCAS 7.4 [53]. For the CASSCF/DFT and CASPT2/DFT embedding calculations, we use a modified version of the MOLCAS code where the embedding interface is adapted from the Molcas-Embed interface developed by Carter and co-workers $[23,54]$. We use stateaveraged CASSCF wave functions with equal weights on the ground and the excited states of interest, and report below the single-state CASPT2 excitation energies. In the CASPT2 calculations, we employ the default IPEA zero-order Hamiltonian [55] and introduce an additional constant imaginary shift [56] of 0.1 a.u. In the CASPT2 calculations, we freeze the lowest $\sigma$ orbitals corresponding to the $1 s$ atomic orbitals of the heavy atoms in the system. We use the Cholesky decomposition of the twoelectron integrals [57] with the threshold of $10^{-4}$. The default convergence criteria are used for all calculations.

In all CASSCF and CASPT2 calculations, the aug-cc-pVDZ [42,58] basis set is used for the solvated molecule and, in the supermolecular calculations, a 6-31G [59] basis set is employed for the solvent molecules. To test the basis-set convergence, we compute the CASPT2 excitation energies of the $p$-nitroaniline with state-independent embedding potentials (case A) with the aug-cc-pVTZ basis set $[42,58]$ and find a decrease by at most $0.05 \mathrm{eV}$ with respect to the use of the aug-cc-pVDZ.

In the QM/MM calculations, we use the TIP3P [60] values of $\mathrm{q}_{\mathrm{O}}=-0.834 e$, $\mathrm{q}_{\mathrm{H}}=0.417 e$ when treating the water molecules as non-polarizable point charges.

\subsection{Results}

To illustrate the performance of the WF/DFT embedding schemes discussed above, we consider the four molecules from Figure 3.1, that is, $p$-nitroaniline (PNA), s-cis-

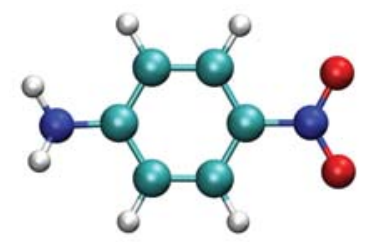

a)

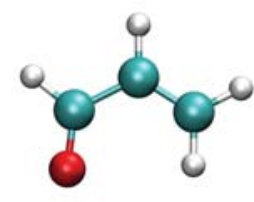

b)

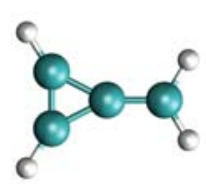

c)

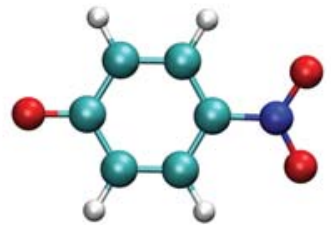

d)

Figure 3.1: The solvated molecules considered in this work: a) $p$-nitroaniline (PNA), b) $s$-cis-acrolein, c) methylenecyclopropene (MCP), and d) $p$-nitrophenolate (PNP). 


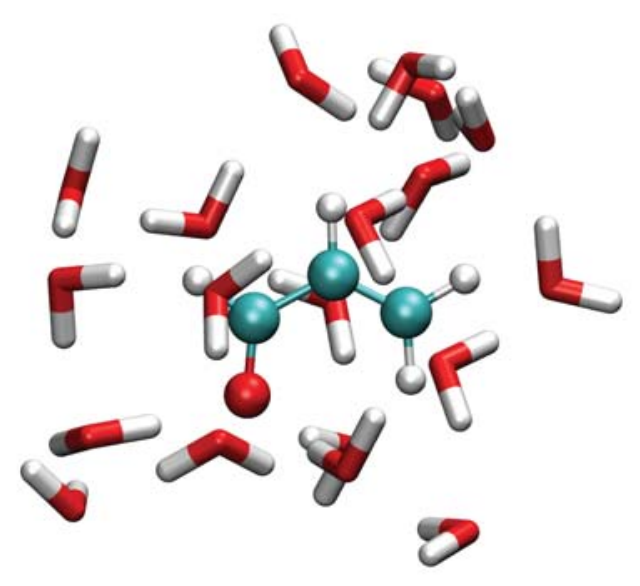

a)

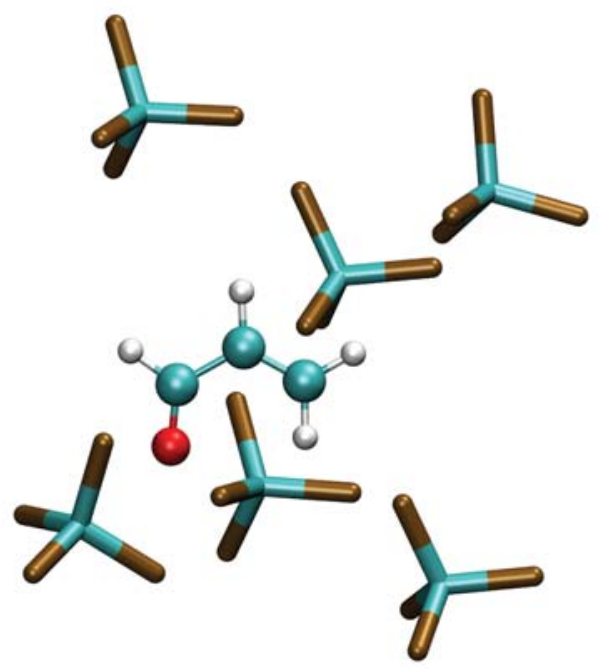

c)
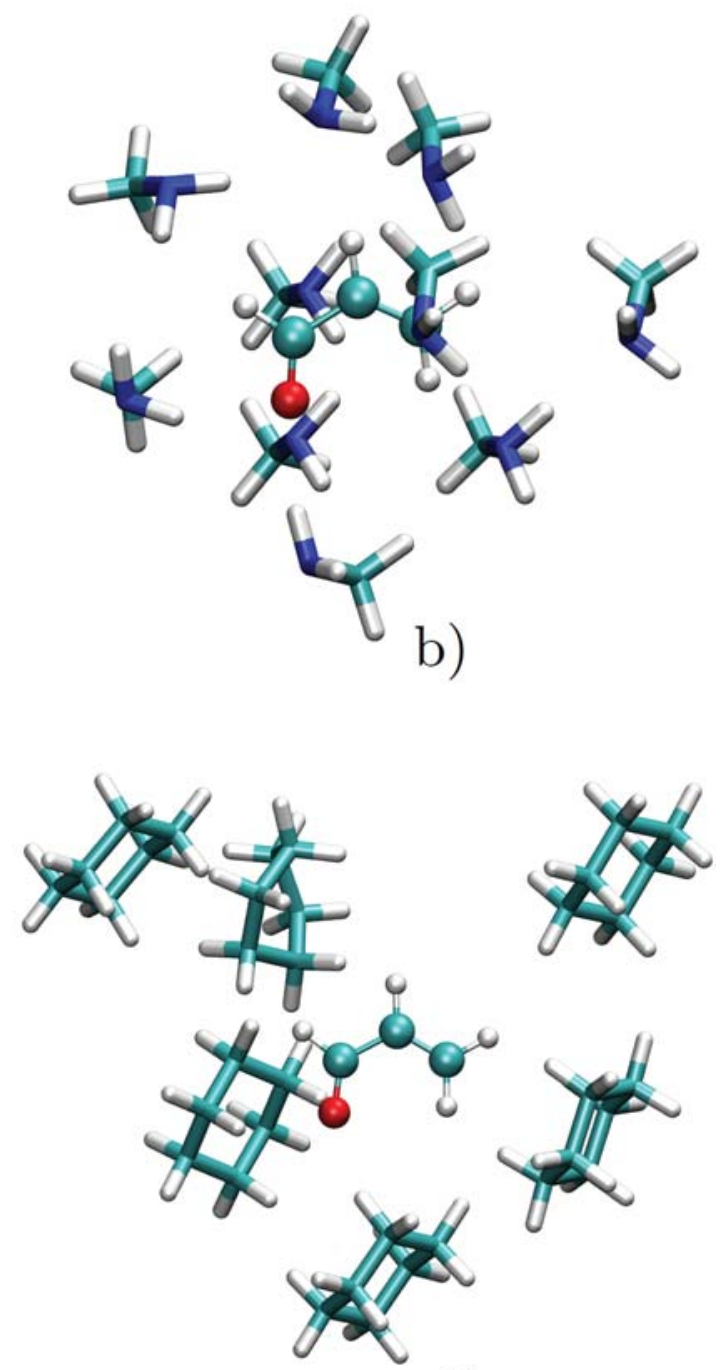

d)

Figure 3.2: Cluster models with acrolein solvated in a) water, b) methylamine $\left(\mathrm{CH}_{3} \mathrm{NH}_{2}\right)$, c) carbon-tetrachloride $\left(\mathrm{CCl}_{4}\right)$, and d) cyclohexane $\left(\mathrm{C}_{6} \mathrm{H}_{12}\right)$. 
acrolein, methylenecyclopropene (MCP), and the anionic p-nitrophenolate (PNP). For each molecule, we construct a model cluster consisting of the molecule solvated in a small number of waters as detailed in Table 3.1. For acrolein, we also consider as solvents methylamine $\left(\mathrm{CH}_{3} \mathrm{NH}_{2}\right)$, carbon-tetrachloride $\left(\mathrm{CCl}_{4}\right)$, and cyclohexane $\left(\mathrm{C}_{6} \mathrm{H}_{12}\right)$. The cluster models for acrolein in the four solvents are shown in Table 3.2.

The choice of these four molecules is motivated by different reasons. PNA represents a perfect test case for our study since its lowest singlet $\pi \rightarrow \pi^{*}$ excitation is characterized by strong intramolecular charge transfer and the response of the solvent environment is expected to be significant, leading to a shift of about $1 \mathrm{eV}$ in the excitation energy in water solution with respect to the gas phase [61,62]. Acrolein is an interesting molecule since it has both conjugated double bonds and lone-pair electrons with the two lowest singlet excitations being of different $n \rightarrow \pi^{*}$ and $\pi \rightarrow \pi^{*}$ character. Furthermore, these two transitions respond in an opposite fashion to a polar solvent like water, with the corresponding excitation energies displaying a blue and a red shift, respectively, with respect to the gas phase [63]. The MCP molecule is also a potentially promising test case since its properties change rather dramatically upon photoexcitation. For instance, in the vertical $\pi \rightarrow \pi^{*}$ transition, the dipole moments of the ground and excited states have opposite signs and the solvatochromic shift in water is expected to be significant and of the order of $0.5 \mathrm{eV}$ [64]. Finally, the anionic PNP has been recently studied with the aid of polarizable force fields and it has been reported that the mutual polarization between PNP and the water solvent accounts for a large part $(0.2-0.3 \mathrm{eV})$ of the shift in the excitation energy with respect to the gas phase [9].

As WF method, we employ the multi-reference perturbation theory approach (CASPT2) both to compute the excitation energies of the solvated molecule in an embedding potential as well as to perform the so-called supermolecular calculations, where the solvation shell is included in the active region and treated at the WF level. In the systems considered here, the excitations are predominantly localized on the solvated molecules and are therefore not characterized by charge transfer to the solvent. Consequently, also in the supermolecular calculations, the excitations can be described with the use of a CAS expansion constructed from a set of orbitals mainly localized on the molecule itself.

For all $\pi \rightarrow \pi^{*}$ excitations, we employ a minimal CAS expansion comprising all $\pi$ electrons in the reference and a number of active orbitals equal to the number of heavy atoms as obtained by using one atomic orbital of $p$ character per heavy atom. For acrolein, since we compute both the $n \rightarrow \pi^{*}$ and the $\pi \rightarrow \pi^{*}$ excitation energies, we use at least 3 states in the state-averaged (SA) calculations and extend the active space to include two lone-pair electrons on the oxygen as well as two additional $n$ and $\pi$ antibonding orbitals. For a given system, we employ the same active space and number of states in the state-averaged calculations both in the subsystem and in the supermolecular calculations as detailed in Table 3.1. For acrolein in cyclohexane, we employ 3 and 5 states in the supermolecular and embedding calculations, respectively, since the relevant states in the latter become the second and the fourth. 


\begin{tabular}{lclcc}
\hline Solute & \multicolumn{2}{c}{ Solvent } & $\mathrm{CAS}(n, m)$ & $\mathrm{SA}$ \\
\hline PNA & 22 & $\mathrm{H}_{2} \mathrm{O}$ & 12,10 & 2 \\
acrolein & 19 & $\mathrm{H}_{2} \mathrm{O}$ & 6,7 & 3 \\
& 10 & $\mathrm{CH}_{3} \mathrm{NH}_{2}$ & 6,7 & 3 \\
& 6 & $\mathrm{CCl}_{4}$ & 6,7 & 3 \\
& 6 & $\mathrm{C}_{6} \mathrm{H}_{12}$ & 6,7 & 3,5 \\
MCP & 17 & $\mathrm{H}_{2} \mathrm{O}$ & 4,4 & 2 \\
PNP & 26 & $\mathrm{H}_{2} \mathrm{O}$ & 12,10 & 2 \\
\hline
\end{tabular}

Table 3.1: Solute molecules and corresponding solvents. The number of solvent molecules included in the solute-solvent cluster model is indicated. We also list the dimension of the CAS expansion with the number $n$ of electrons in $m$ active orbitals, which we employ in the wave function calculations, and the number of states included in the state-averaged (SA) calculations.

\subsubsection{Excited-state DFT density}

The construction of the embedding potential in the WF/DFT calculations requires an approximation for the density of both the active system and the environment. In case (A), we use the same strategy as in Ref. [37], i.e., we utilize the densities obtained in a subsystem DFT treatment for the ground state of both systems. In case (B), we want to relax the environment within subsystem DFT in the presence of a solvated molecule in its excited state, and must therefore construct an approximate DFT density of the excited state of the solute. Here, we test two simple procedures, which lead to two different approximate densities named here self-consistent postKohn-Sham (scp-KS) and fully relaxed (fr-KS) densities.

In both schemes for case (B), we assume that the excitation is of pure singleorbital transition nature. We therefore start from the ground-state Kohn-Sham orbitals and modify the occupation numbers by moving an electron from an occupied to a virtual orbital with a 0.5 fractional occupation of the up- and down-spin components in both orbitals. Here, we generally focus on the lowest singlet excitation where one electron is promoted from the HOMO to the LUMO orbital. The only exception is the $n \rightarrow \pi^{*}$ excitation in acrolein, which is a transition from the HOMO-1 to the LUMO orbital at the KS level. In the scp-KS scheme, the orbitals of the molecules are not further relaxed, so the excited-state density is simply constructed with fractionally occupied ground-state orbitals. In the fr-KS case, we relax the orbitals at fixed fractional occupations until convergence.

We then keep the molecule in the chosen excited-state density and relax the environment in the ground state. Subsequently, we freeze the environment and find a new excited-state density of the solvated molecule by relaxing the orbitals either in the ground state or with fixed fractional occupations for the scp-KS and the fr-KS case, respectively. We repeat these steps (generating the excited-state density and relaxing the environment around the excited molecule) in cycles until the densities of the environment and of the solvated molecule are converged. We find that 5-6 cycles suffice for convergence for all systems. 


\subsubsection{Nitroaniline and acrolein}

We employ the WF/DFT embedding schemes with a state-independent (A) and a state-specific (B) embedding potential to compute the excitation energies $\Delta E^{(A)}$ and $\Delta E^{(B)}$ (Eqs. 3.24 and 3.26) of solvated PNA and acrolein. For scheme (B), we compute two sets of WF/DFT excitation energies corresponding to the two different embedding potentials we generate with the use of the scp-KS and the fr-KS excitedstate DFT density for the active region. In all cases, we also evaluate the corrections which must be added to the simple excitation energies obtained as eigenvalue differences of the solute Hamiltonian including the embedding potential (Eqs. 3.17 and 3.27). We denote these energy contributions as

$$
\begin{aligned}
& \delta E_{\text {tot }}^{(\mathrm{A})}=\delta E_{\text {nadd }}^{(\mathrm{A})} \\
& \delta E_{\text {tot }}^{(\mathrm{B})}=\delta E_{\text {nadd }}^{(\mathrm{B})}+\delta E_{\text {simple }}^{(B)},
\end{aligned}
$$

where we make use of Eqs. 3.25, 3.31, and 3.32. Then, in the case of water solvent, for which standard force fields are available, we consider a conventional QM/MM approach where the solvation shell is represented in terms of a non-polarizable pointcharge model as it is often done for complex biological or solvated systems. Finally, we perform a supermolecular calculation where the whole system is treated at the WF level, which provides a reference value for the other types of embedding. We collect all results in Table 3.2.

For PNA in water, we consider three structures of the model cluster corresponding to different steps during the geometry optimization, with the last structure (geometry 3 ) being the converged geometry of lower energy. For all structures, we find that already scheme (A) gives a better accuracy than the simple point-charge model, reducing the excitation energies by about $0.1-0.15 \mathrm{eV}$ and bringing them in better agreement with the supermolecular values. The use of scheme (B) in the variant which includes the correction to the eigenvalue difference of the state-specific WF calculations further improves the excitation energies. On the other hand, the neglect of the corrections in scheme (B) would yield significantly worse results. In fact, while these terms are rather small in case (A) where they only include differential non-additive contributions (Eqs. 3.25), they can instead be non negligible in case (B). Furthermore, they also appear to depend rather strongly on the particular choice of excited-state DFT density for the generation of the excited-state embedding potential, being consistently smaller in the case of the so-called fr-KS density. Perhaps surprisingly, the excitation energies in scheme (B) including these corrections are instead rather independent of the particular choice of excited-state DFT density employed.

The best performance of scheme (B) is observed in correspondence of geometry 1. To understand how (B) improves upon (A), we can inspect the difference $v_{\mathrm{emb}, 1}^{\text {pol,e }}(\mathbf{r})-v_{\mathrm{emb}, 1}(\mathbf{r})$ between the excited-state embedding potential used in the WF excited-state calculation of case (B) and the ground-state potential used both in case (A) and in the WF ground-state calculation of case (B), which is shown in Figure 3.3. We observe that the excited-state potential is more negative than the ground-state potential in the region close to the nitro group while the opposite holds in proximity 


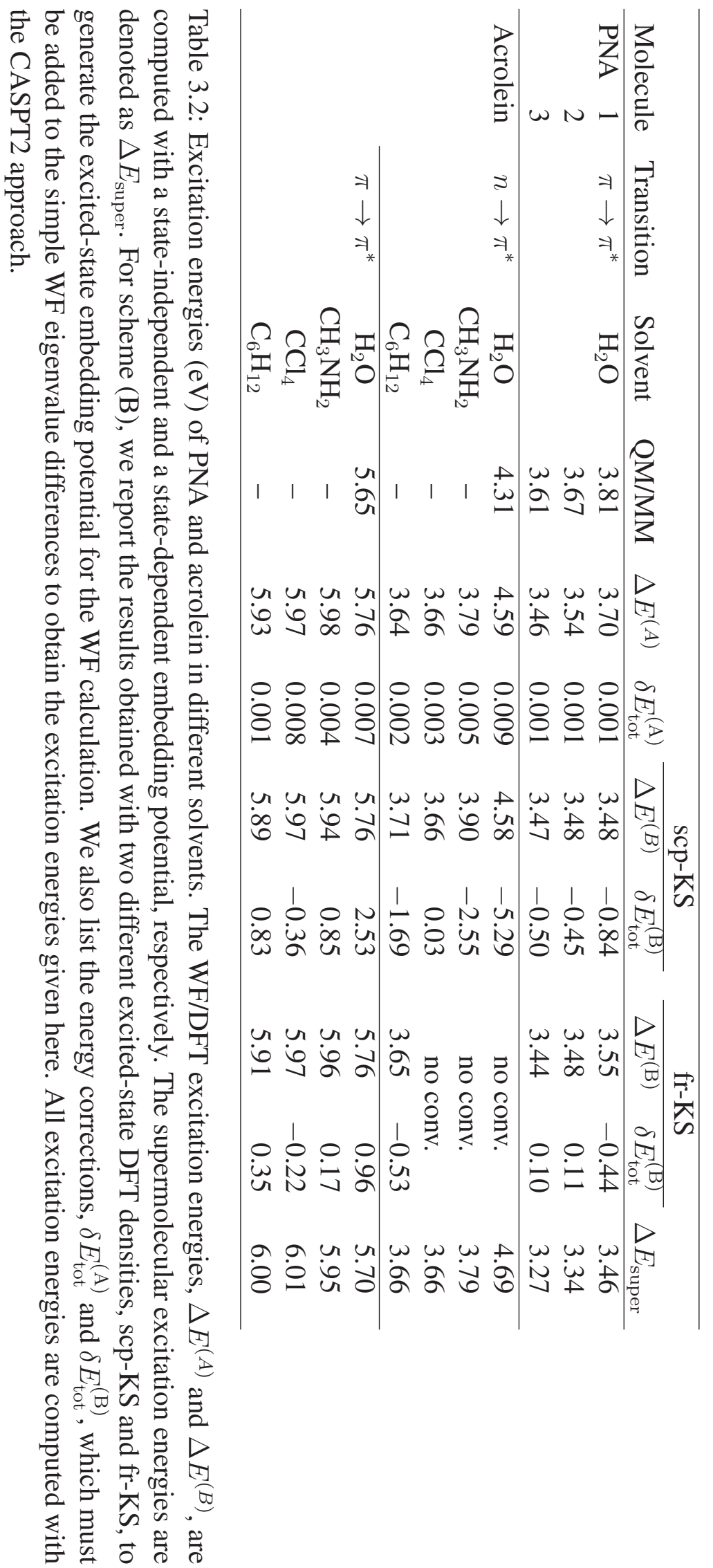




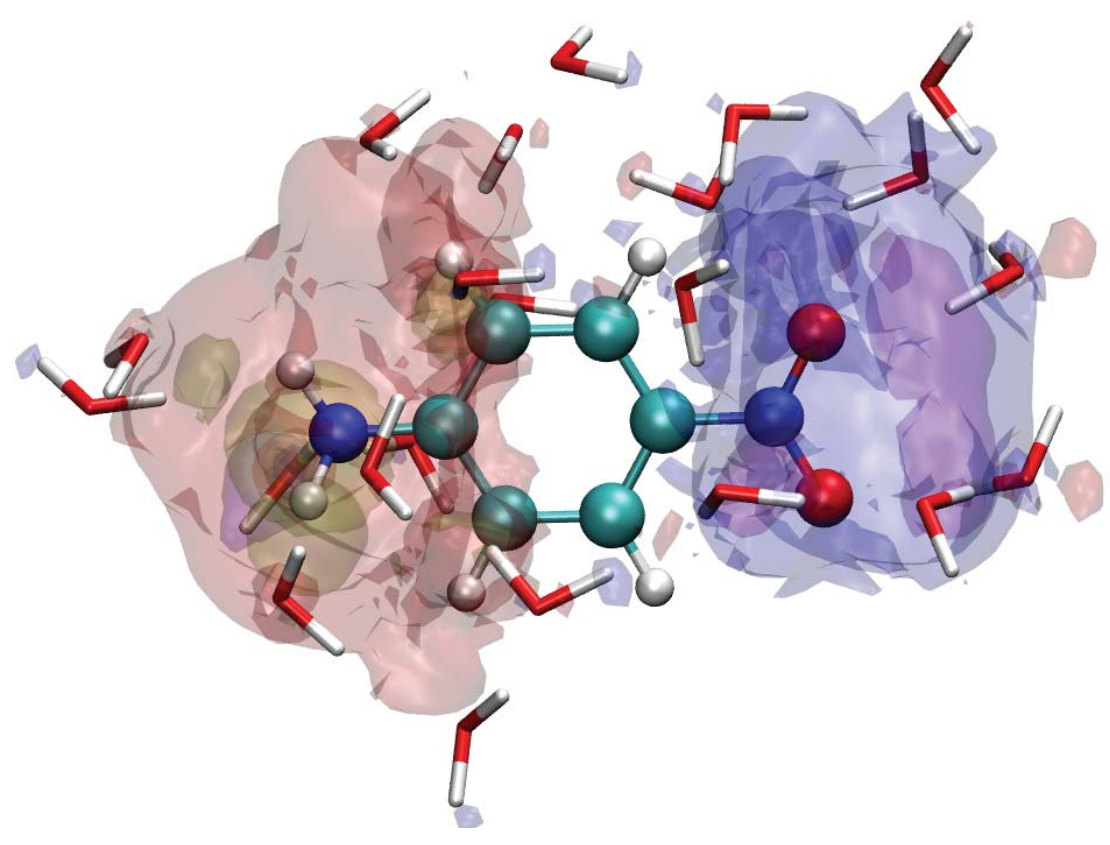

Figure 3.3: Difference of the state-specific excited- and ground-state embedding potentials for PNA (geometry 1). The excited-state potential is obtained with the use of the scp-KS excited-state density. The contours in violet, blue, red, and yellow correspond to the values of $-0.02,-0.01,+0.01$, and +0.02 a.u., respectively.

of the amine group. Consequently, since the excitation implies a transfer of charge from the amine to the nitro group, the excited-state embedding potential will stabilize the excited state more than the ground-state, resulting in a lower excitation energy in scheme (B) than in (A).

An analysis of the results for structures 2 and 3 leads to qualitatively similar observations, but the improvement of the corrected case (B) compared to case (A) is diminished along the sequence of structures 1, 2, 3. In particular, for geometry 3 , the (A) and (B) excitation energies are very similar, superior to the QM/MM value but about $0.2 \mathrm{eV}$ higher than the supermolecular result. Since geometries 1, 2, and 3 represent a succession of structures along a geometry optimization with the last one being a minimum, it is possible that the nature of the hydrogen bonds changes as the structure reaches equilibrium, rendering the partitioning in different regions not adequate to the present case. To investigate this hypothesis, we compute the QM/MM, (A), and (B) excitation energies of structure 3, including some water molecules in the active region which are depicted in Figure 3.4. As reported in Table 3.3, when the waters close to the amino group are included in the active region and treated at the WF level, the agreement with the supermolecular calculation improves and the (A) and (B) excitation energies become well within $0.1 \mathrm{eV}$ of the supermolecular value, with (B) being in better agreement that (A). Therefore, as conjectured, moving the partition between the two parts further away from the active systems and allowing 
a better description of relevant hydrogen bonds significantly improves the performance of the (A) and, in particular, the (B) scheme. Finally, while including these water molecules in the active part also improves the QM/MM excitation energy, the $\mathrm{QM} / \mathrm{MM}$ value remains $0.24 \mathrm{eV}$ higher than the supermolecular reference.

\begin{tabular}{ccccc}
\hline & & & \multicolumn{2}{c}{$\Delta E_{\mathrm{B}}$} \\
\cline { 4 - 5 } Active $\mathrm{H}_{2} \mathrm{O}$ & $\mathrm{QM} / \mathrm{MM}$ & $\Delta E_{\mathrm{A}}$ & scp-KS & fr-KS \\
\hline 0 & 3.61 & 3.46 & 3.47 & 3.44 \\
3 & 3.51 & 3.36 & 3.34 & 3.32 \\
22 (all) & 3.27 & 3.27 & 3.27 & 3.27 \\
\hline
\end{tabular}

Table 3.3: Excitation energies (eV) of PNA in water (geometry 3), computed with different numbers of water molecules included in the active region and treated at the WF level. The supermolecular computation is the limit of all four methods when all 22 water molecules are in the active system. For the notation, see caption of Table 3.2.

In 3.2, we also list the (A) and (B) excitation energies of acrolein in water and in the three other solvents shown in Figure 3.2. For case (B), we compute the excitedstate potentials starting from the scp-KS and the fr-KS excited-state DFT densities. Perhaps not surprisingly, we find that the second procedure presents problems of convergence for the $n \rightarrow \pi^{*}$ excitation, where the construction of the density does not follow the Aufbau principle. We do instead not encounter such problems in the case of the $\pi \rightarrow \pi^{*}$ excitation, which corresponds to the HOMO-LUMO transition in KS-DFT.

For the $\pi \rightarrow \pi^{*}$ transition of acrolein in all solvents, the two sets of (B) excitation energies are always similar, and rather close to both the (A) and the supermolecular values. Therefore, differential polarization does not appear to play a significant role in the description of this transition even though some of the solvents would have been expected to strongly interact with the $\pi$ electronic system of acrolein. This observation can be extended to the $n \rightarrow \pi^{*}$ transition where the (A) excitation energies are already in good agreement with the corresponding supermolecular calculation. In fact, for the $\pi \rightarrow \pi^{*}$ case, the use of scheme (B) with the scp-KS density slightly worsens the agreement with the reference values in the case of acrolein solvated in methylamine and cyclohexane. We note that, for cyclohexane, the use of a statespecific excited-state embedding potential constructed with the fully relaxed DFT excited-state density improves upon the scp-KS-based result, restoring the (A) value and the good agreement with the supermolecular calculation. We will further comment on the choice of excited-state DFT density for the construction of state-specific potentials below.

Finally, the energy corrections used in scheme (B) are always substantial for acrolein, being as large as $5 \mathrm{eV}$ for $n \rightarrow \pi^{*}$ transition in water and the scp-KS case. Consequently, the excitation energies computed without these corrections as the eigenvalue difference of the state-specific Hamiltonians of the solute would be totally unreasonable. We note that the corrections to compute the (B) excitation energies based on the fr-KS density are systematically smaller that those based on the 


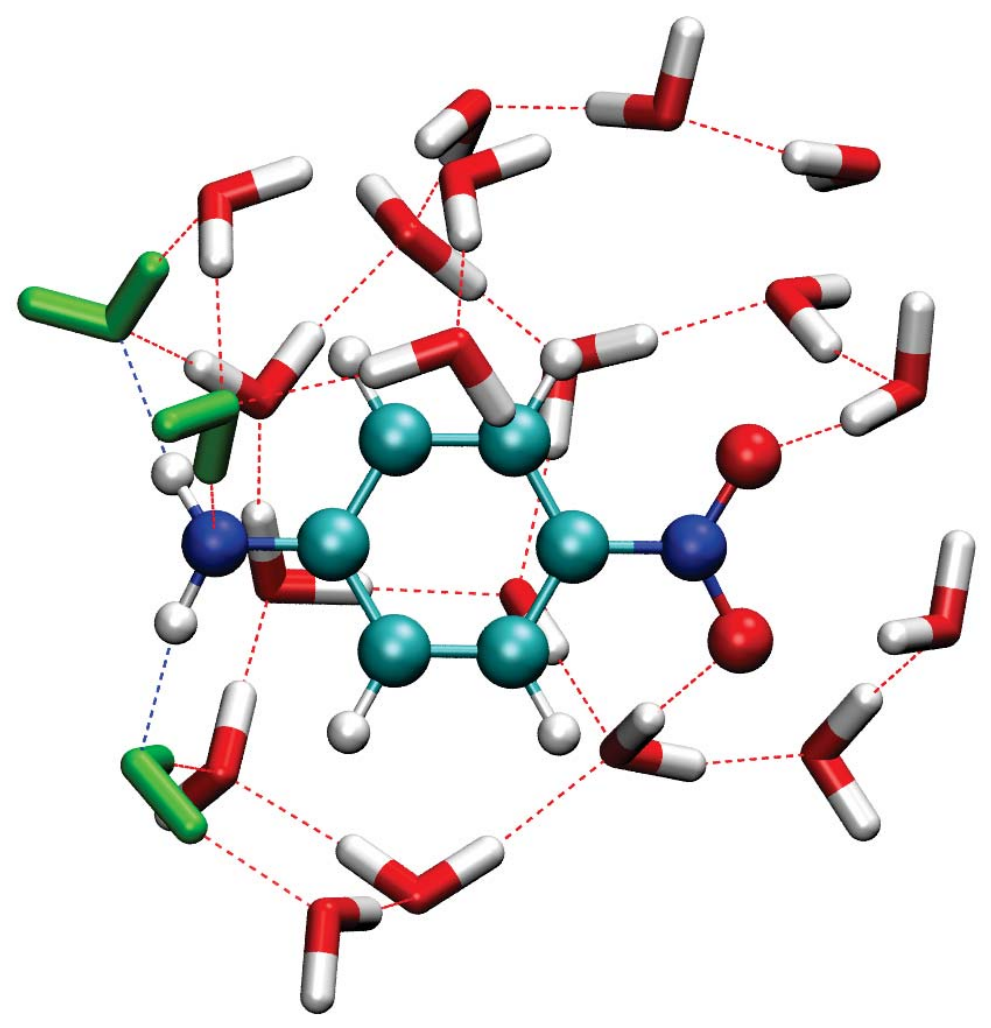

Figure 3.4: PNA and its solvation shell of 22 water molecules for structure 3. We depict in green the three water molecules added to the active region in Table 3.3.

scp-KS density. Finally, as for PNA, the use of eigenvalue differences of the solute Hamiltonian including the embedding potential is justified for scheme (A) since the energy corrections (computed as differences of non-additive quantities) are always smaller than $0.01 \mathrm{eV}$.

\subsubsection{Correction and non-additive contributions}

In Table 3.4, we analyze in more detail the energy corrections (Eqs. 3.31 and 3.32) which must be added to the eigenvalue differences of the state-specific WF calculations to obtain the final (B) excitation energy. In addition to PNA in water and acrolein in the four different solvents, we consider the MCP and PNP molecules in water, and focus on the excitation energies obtained with the use of the scp-KS excited-state density since it is possible to converge this DFT density for all cases reported in the table.

We observe that, similarly to what was found for scheme (A), the non-additive contributions are rather small, less than $0.02 \mathrm{eV}$, also in scheme (B). Therefore, the largest component in the energy corrections to the eigenvalue difference of statespecific solute Hamiltonians comes from the KS energy difference of the environment, being polarized in the ground and the excited state of the active part, plus the 


\begin{tabular}{lccccrrr}
\hline Solute & Transition & Solvent & $\Delta E_{\text {approx }}^{(\mathrm{B})}$ & $\delta E_{\text {simple }}^{(B)}$ & $\delta E_{\text {nadd }}^{(\mathrm{B})}$ & $\Delta E^{(\mathrm{B})}$ \\
\hline PNA & 1 & $\pi \rightarrow \pi^{*}$ & $\mathrm{H}_{2} \mathrm{O}$ & 4.32 & -0.85 & 0.005 & 3.48 \\
& 2 & & & 3.93 & -0.46 & 0.005 & 3.48 \\
& & & 3.98 & -0.51 & 0.005 & 3.47 \\
\hline Acrolein & $n \rightarrow \pi^{*}$ & $\mathrm{H}_{2} \mathrm{O}$ & 9.88 & -5.27 & -0.023 & 4.58 \\
& & $\mathrm{CH}_{3} \mathrm{NH}_{2}$ & 6.46 & -2.54 & -0.013 & 3.90 \\
& & $\mathrm{CCl}_{4}$ & 3.62 & 0.03 & 0.003 & 3.66 \\
& & $\mathrm{C}_{6} \mathrm{H}_{12}$ & 5.41 & -1.68 & -0.007 & 3.71 \\
\cline { 3 - 7 } & $\pi \rightarrow \pi^{*}$ & $\mathrm{H}_{2} \mathrm{O}$ & 3.23 & 2.54 & -0.012 & 5.76 \\
& & $\mathrm{CH}_{3} \mathrm{NH}_{2}$ & 5.08 & 0.85 & -0.002 & 5.94 \\
& & $\mathrm{CCl}_{4}$ & 6.33 & -0.36 & 0.001 & 5.97 \\
& & $\mathrm{C}_{6} \mathrm{H}_{12}$ & 5.07 & 0.83 & -0.004 & 5.89 \\
\hline PCP & $\pi \rightarrow \pi^{*}$ & $\mathrm{H}_{2} \mathrm{O}$ & 5.18 & -0.48 & -0.045 & 4.66 \\
\hline & 1 & $\pi \rightarrow \pi^{*}$ & $\mathrm{H}_{2} \mathrm{O}$ & 17.03 & -12.75 & 0.003 & 4.28 \\
& & & 16.45 & -12.29 & 0.006 & 4.17 \\
& & & & 14.95 & -10.85 & 0.010 & 4.11 \\
\hline
\end{tabular}

Table 3.4: Excitation energies (eV) computed with scheme (B). The energy corrections are split in the non-additive and remaining contributions, which must be added to the eigenvalue differences of state-specific WF calculations $\left(\Delta E_{\mathrm{approx}}^{(\mathrm{B})}\right)$ to obtain the correct excitation energies $\left(\Delta E^{(\mathrm{B})}\right)$. The scp-KS excited-state DFT density is used in the generation of the state-specific embedding potential.

difference of the nuclear interaction of the active fragment with these two environmental densities. This contribution can be remarkably large and, in the case of PNP in three different water clusters, amounts to as much as $-11 \mathrm{eV}$, greatly exceeding in absolute value the (B) excitation energy of about $4 \mathrm{eV}$. It is remarkable that the addition of such large energy corrections to a grossly incorrect eigenvalue difference leads to very reasonable excitation energies. As already mentioned and also shown below, it is even more remarkable that different procedures to compute the excitedstate density in the generation of the state-specific potentials leads to very different eigenvalue differences and energy corrections, but remarkably close final excitation energies.

\subsubsection{Construction of excited-state DFT density}

We discuss here in more detail the choice of excited-state density for the active site employed to polarize the other fragments and construct a state-specific embedding potential for the WF excited-state calculation. To understand how this choice affects the the (B) excitation energies, we consider the MCP and PNP molecules in water solvent and, in addition to the scp-KS and the fr-KS densities, we construct excitedstate densities of the active region with fractional occupations over the HOMO-1, HOMO, LUMO, and LUMO+1 orbitals. The occupation numbers are based on the occupations of the natural orbitals of the excited-state wave function obtained in the 


\begin{tabular}{llcccccrr}
\hline Method & Density & $\mathrm{H}-1$ & $\mathrm{H}$ & $\mathrm{L}$ & $\mathrm{L}+1$ & $\Delta E$ & $\delta E_{\text {simple }}$ & $\delta E_{\text {nadd }}$ \\
\hline Super & & - & - & - & - & 5.03 & - & - \\
QM/MM & & - & - & - & - & 4.88 & - & - \\
A & & 2 & 2 & 0 & 0 & 5.08 & - & 0.017 \\
B & scp-KS & 2 & 1 & 1 & 0 & 4.66 & -0.48 & -0.045 \\
B & fr-KS (1) & 2 & 1 & 1 & 0 & 4.81 & 0.12 & -0.035 \\
B & fr-KS (2) & 1.9 & 1 & 1 & 0.1 & 4.87 & -0.82 & -0.036 \\
& & & & PNP & & & & \\
Super & & - & - & - & - & 3.50 & - & - \\
QM/MM & & - & - & - & - & 3.51 & - & - \\
A & & 2 & 2 & 0 & 0 & 3.49 & - & 0.001 \\
B & scp-KS & 2 & 1 & 1 & 0 & 4.11 & -10.85 & 0.010 \\
B & fr-KS (1) & 2 & 1 & 1 & 0 & 3.72 & -4.79 & 0.009 \\
B & fr-KS (2) & 1.9 & 1.2 & 0.8 & 0.1 & 3.67 & -4.07 & 0.007 \\
\hline
\end{tabular}

Table 3.5: Excitation energies and energy corrections (non-additive and remaining contributions) of the MCP and PNP (geometry 3) molecules in water solvents, obtained in a supermolecular, QM/MM, (A), and (B) calculation. In scheme (B), we employ the scp-KS and fr-KS excited-state densities obtained with (1) standard HOMO-LUMO and (2) fractional occupations over the HOMO-1, HOMO, LUMO, and LUMO+1 KS orbitals. The corresponding natural-orbital occupation numbers of the excited-state CASSCF wave function obtained in scheme (A) are (1.94, 1.01, $1.00,0.06)$ and $(1.88,1.17,0.87,0.12)$ for MCP and PNP, respectively.

CASSCF calculations and the KS density is then relaxed in the spirit of the fr-KS density introduced before. The results are summarized in Table 3.5. We stress that the densities with fractional occupations are constructed to test the sensitivity of the result to the choice of approximate excited-state DFT density and are not proposed as a general practical route for scheme (B).

For both molecules, the (A) excitation energy is in very good agreement with the corresponding supermolecular calculation and even an MM description of the environment appears to be sufficiently accurate. Therefore, differential polarization effects due to the response of the environment to the excitation of the active region are surely expected to be negligible. Nevertheless, scheme (B) with the use of the scpKS density modifies the (A) excitation energy, significantly worsening the agreement of the WF/DFT approach with the reference. To understand whether this is a fundamental failure of scheme (B) or a problem with the construction of the approximate excited-state density needed in scheme (B), we compute the (B) excitation energies based on (1) the HOMO-LUMO fully relaxed density employed also above and (2) the fully relaxed density with fractional occupations. We find that the (B) excitation energy improves as we move from the scp-KS to the fr-KS (1) and the fr-KS (2) density, with the error with respect to the supermolecular value being reduced from 0.4-0.5 eV to less than $0.2 \mathrm{eV}$. Finally, as a consistency check, we consider two cases, 
PNA and the $\pi \rightarrow \pi^{*}$ transition of acrolein, where scp-KS and fr-KS densities yield similar (B) excitation energies and verify the robustness of this agreement by also constructing fully relaxed excited-state densities with fractional occupations inspired by the CASSCF wave functions. As shown in Table 3.6, the use of fractional occupations in the excited-state DFT density does not affect the (B) excitation energy of these two systems, supporting the idea that the equivalent performance of the scp-KS and fully relaxed HOMO-LUMO density is a good indicator of the robustness of the (B) results for the particular system under study.

\begin{tabular}{|c|c|c|c|c|c|c|c|c|}
\hline Method & Density & H-1 & $\mathrm{H}$ & $\mathrm{L}$ & $\mathrm{L}+1$ & $\Delta E$ & $\delta E_{\text {simple }}$ & $\delta E_{\text {nadd }}$ \\
\hline \multicolumn{9}{|c|}{ PNA (geometry 3) } \\
\hline QM/MM & & - & - & - & - & 3.61 & - & - \\
\hline A & & 2 & 2 & 0 & 0 & 3.46 & - & 0.001 \\
\hline B & scp-KS & 2 & 1 & 1 & 0 & 3.47 & -0.51 & 0.005 \\
\hline B & fr-KS (1) & 2 & 1 & 1 & 0 & 3.44 & 0.09 & 0.005 \\
\hline B & fr-KS (2) & 1.90 & 1.30 & 0.70 & 0.10 & 3.46 & -0.80 & -0.011 \\
\hline Super & & - & - & - & - & 3.27 & - & - \\
\hline \multicolumn{9}{|c|}{ Acrolein $\left(\pi \rightarrow \pi^{*}\right)$} \\
\hline QM/MM & & - & - & - & - & 5.65 & - & - \\
\hline A & & 2 & 2 & 0 & 0 & 5.76 & - & 0.007 \\
\hline B & scp-KS & 2 & 1 & 1 & 0 & 5.76 & 2.54 & -0.012 \\
\hline B & fr-KS (1) & 2 & 1 & 1 & 0 & 5.76 & 0.98 & -0.012 \\
\hline B & fr-KS (2) & 1.97 & 1.03 & 1 & 0 & 5.76 & 0.90 & -0.012 \\
\hline Super & & - & - & - & - & 5.70 & - & - \\
\hline
\end{tabular}

Table 3.6: Excitation energies and energy corrections (non-additive and remaining contributions) of the PNA and acrolein $\left(\pi \rightarrow \pi^{*}\right.$ transition) molecules in water solvents, obtained in a supermolecular, $\mathrm{QM} / \mathrm{MM},(\mathrm{A})$, and (B) calculation. In scheme (B), we employ the scp-KS and fr-KS excited-state densities obtained with (1) standard HOMO-LUMO and (2) fractional occupations over the HOMO-1, HOMO, LUMO, and LUMO+1 KS orbitals. The corresponding natural orbital occupation numbers of the excited-state CASSCF wave function obtained in scheme (A) are $(1.90,1.26,0.78,0.11)$ for PNA and $(1.95,1.04,0.96,0.03)$ for acrolein (note that, since the CAS has more than 4 orbitals, the CASSCF occupations do not need to sum to 4 while, for DFT, they necessarily do). Since the LUMO+1 orbital for acrolein has a different character in the CASSCF and KS calculations, we only use the HOMO-1, HOMO, and LUMO orbitals in the fully relaxed run with fractional occupations.

To investigate more closely the relative accuracy of the scp-KS and fr-KS procedures employed to obtain the excited-state density of the active molecule, we compare the difference between the ground- and excited-state densities computed within subsystem DFT and CASPT2 for MCP in water. As seen above, this molecule represents a good test case since the use of these two excited-state densities leads to different (B) excitation energies, with the values obtained with the fr-KS density being in better agreement with the supermolecular reference. As DFT densities for 


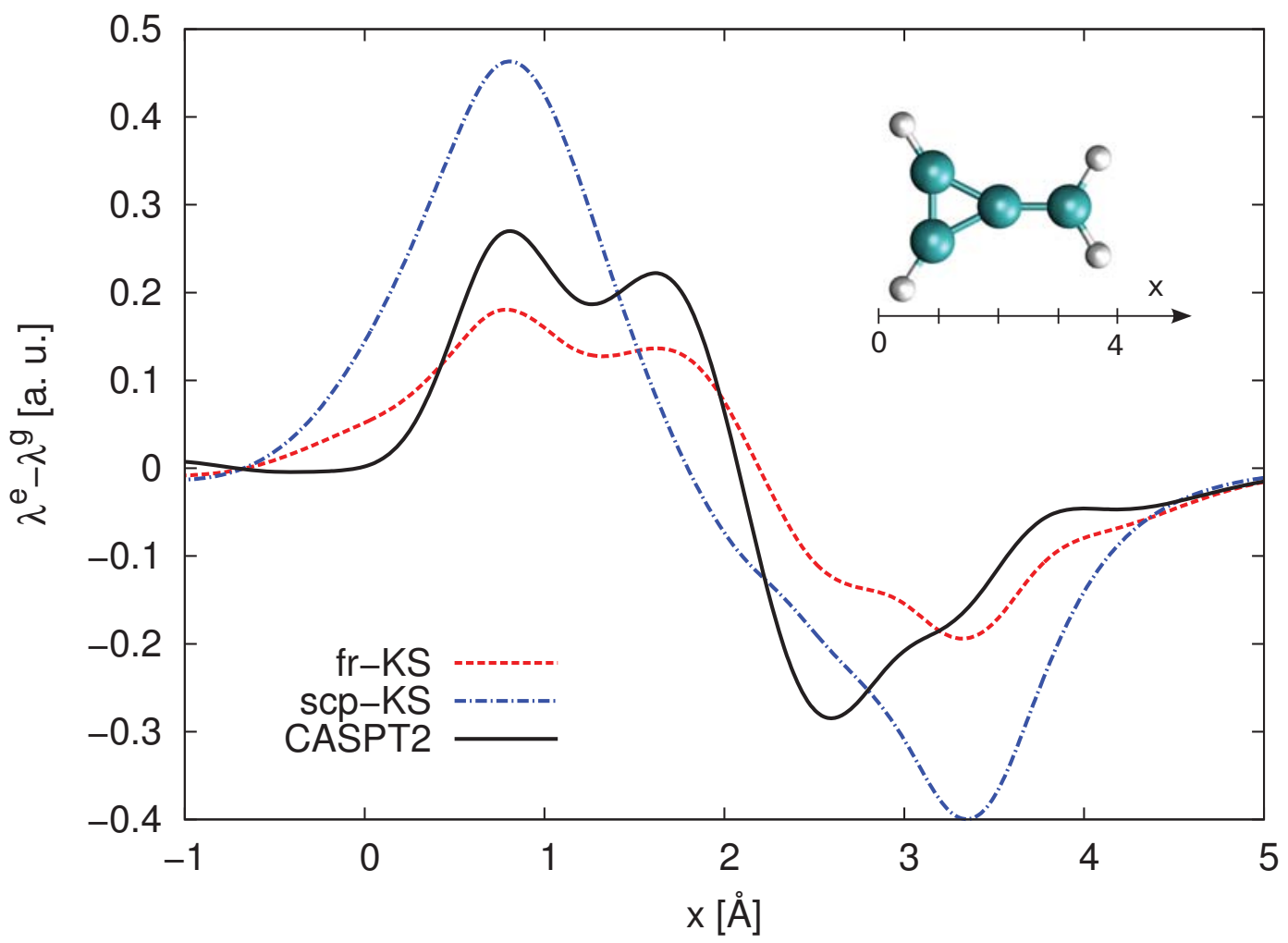

Figure 3.5: Difference between the excited- and ground-state densities of MCP in water, integrated over planes perpendicular to the X-axis (Eq. 3.34) and computed with CASPT2 and the approximate subsystem DFT schemes.

the total system, we use the sum of the densities of the molecule and the environment obtained self-consistently for each subsystem (a comparison of the densities of only the active subsystem yields a very similar plot, not shown here). The CASPT2 densities are computed with the first-order wave function from a supermolecular calculation (and therefore also include some second-order contributions) and are here obtained through perturbative calculations on top of state-specific CASSCF wave functions separately targeting the ground and the excited state. For the vertical excitation of MCP in water, the use of state-specific CASSCF energies is robust as the energy difference between the ground and excited states is large and, furthermore, the corresponding wave functions of the nearly planar molecule preserve approximately different symmetry character. On the other hand, the use of state-average orbitals leads to CASSCF densities of significantly lower quality and therefore to less reliable CASPT2 densities, as discussed in more detail in the Appendix.

In Figure 3.5, we plot the difference between the excited- and ground-state densities integrated over cross-sections normal to the axis formed by the two carbon atoms of the $\mathrm{CCH}_{2}$ terminus (defined here as the $\mathrm{x}$-axis):

$$
\lambda^{e}(x)-\lambda^{g}(x)=\int \rho^{e}(x, y, z) \mathrm{d} y \mathrm{~d} z-\int \rho^{g}(x, y, z) \mathrm{d} y \mathrm{~d} z .
$$

We observe that both approximate DFT densities correctly display the general feature 
that charge transfer occurs from the ethylene moiety to the ring but differ significantly in describing the extent of the change. In particular, the scp-KS procedure leads to a more marked transfer of charge which mainly interests the extremes of the molecule, while the fr-KS density is more structured and better reproduces the general features of the CASPT2 reference. Therefore, the improvement observed in the fr-KS excitation energy of MCP as compared to the scp-KS value coincides with a superior description of the excited-state density employed to construct the state-specific excited-state embedding potential for the WF calculation. Similarly, we have found that also for PNP, the fr-KS density is of better quality than the scp-KS one. It is however clear that alternative strategies must be devised for the construction of the excited-state density since non-negligible differences remain between the fr-KS and the reference density, and between the corresponding (B) excitation energy and the supermolecular value. Furthermore, we must recall that the fr-KS scheme may suffer from convergence problems for non-Aufbau excited-state densities as shown above for the $n \rightarrow \pi^{*}$ excitation of acrolein.

Finally, we observe that the use of different excited-state DFT densities in scheme (B) can lead to quite large and different energy corrections (Eq. 25) as shown in Table 5. For instance, in the case of PNP, the corrections corresponding to the scp-KS and fr-KS densities are -10.85 and $-4.79 \mathrm{eV}$, respectively, and, therefore, differ by as much as $6 \mathrm{eV}$. This remarkable difference is however diminished when the corrections are added to the eigenvalue differences of the state-specific Hamiltonians (Eq. 22). The final excitation energies of PNP in scheme (B) are in fact rather similar for the two choices of excited-state densities, and equal to 4.11 and $3.72 \mathrm{eV}$, respectively.

To understand the reasons behind this cancellation, we rewrite the expression of the eigenvalue difference (Eq. 22) neglecting the small non-additive contributions as

$$
\begin{aligned}
\Delta E_{\text {approx }}^{(B)} & \approx E_{1}^{\mathrm{WF}}\left[\Psi_{1}^{p o l, e}\right]-E_{1}^{\mathrm{WF}}\left[\Psi_{1}^{g}\right] \\
& +V_{\text {nuc }, 2}\left[\rho_{1}^{\text {pol }, e}\right]-V_{\mathrm{nuc}, 2}\left[\rho_{1}^{g}\right]+J\left[\rho_{1}^{\text {pol }, e}, \rho_{2}^{\text {pol }}\right]-J\left[\rho_{1}^{g}, \rho_{2}\right] \\
& =E_{1}^{\mathrm{WF}}\left[\Psi_{1}^{p o l, e}\right]-E_{1}^{\mathrm{WF}}\left[\Psi_{1}^{g}\right]+\int v_{\mathrm{nuc}, 2}(\mathbf{r}) \Delta \rho_{1}(\mathbf{r}) \mathrm{d} \mathbf{r} \\
& +J\left[\rho_{1}^{g}, \Delta \rho_{2}\right]+J\left[\rho_{2}, \Delta \rho_{1}\right]+J\left[\Delta \rho_{1}, \Delta \rho_{2}\right] .
\end{aligned}
$$

where $\Delta \rho_{1}(\mathbf{r})=\rho_{1}^{p o l, e}(\mathbf{r})-\rho_{1}^{g}(\mathbf{r})$. The last term is relatively small compared to the other electrostatic terms and the first WF energy difference is expected to be not so sensitive to changes in the embedding potential, which only affect the expectation value through changes in the excited-state wave function of system 1. Furthermore, the second term describing the interaction of nuclei of system 2 with $\Delta \rho_{1}$ will largely cancel the electrostatic interaction $J\left[\rho_{2}, \Delta \rho_{1}\right]$. Therefore, the only term in the expression above which may significantly vary with changes in $\Delta \rho_{2}$ is $J\left[\rho_{1}^{g}, \Delta \rho_{2}\right]$.

We then observe that the leading term in the energy correction (Eq. 25) is the interaction of the nuclei of the active system 1 with the density difference $\Delta \rho_{2}$ of the environment. We expect this contribution to largely cancel the electrostatic interaction of $\rho_{1}^{g}$ with $\Delta \rho_{2}$ in the equation above, namely,

$$
\int v_{\text {nuc }, 1}(\mathbf{r}) \Delta \rho_{2}(\mathbf{r}) \mathrm{d} \mathbf{r}+J\left[\rho_{1}^{g}, \Delta \rho_{2}\right] \approx 0,
$$


to zeroth order. Therefore, the final excitation energy obtained by adding the energy correction (Eq. 25) to the eigenvalue difference (Eq. 22) will vary less than the correction and the eigenvalue difference itself, when different excited-state DFT densities are employed to polarize the environment and construct the state-specific embedding potential. To illustrate this cancellation in PNP, we note that the interaction of the nuclei of system 1 with $\Delta \rho_{2}$ is equal to -10.74 and $-4.69 \mathrm{eV}$ in correspondence of the scp-KS and the fr-KS excited-state density, and amounts to almost the full energy correction of -10.85 and $-4.79 \mathrm{eV}$, respectively. Furthermore, these contributions largely cancel the corresponding terms, $J\left[\rho_{1}^{g}, \Delta \rho_{2}\right]$, which are of opposite sign and equal to 11.10 and $4.85 \mathrm{eV}$.

\subsection{Conclusions}

In this work, we have outlined a practical route to compute the excitation energies of a molecule embedded in a responsive environment within a WF/DFT scheme that can account for the mutual polarization of the fragments upon excitation of the active molecule. The approach relies on the construction of state-specific embedding potentials within an approximate subsystem DFT approach we have adapted to treat the active fragment in its excited state. We have evaluated the explicit energy expression of the total system in the ground and excited states, using both state-independent and state-dependent embedding potentials, that is, either freezing or relaxing the environment in response to the excitation of the embedded molecule.

We showed that, if state-independent embedding potentials are used (frozen environment), computing the excitation energies as WF eigenvalue differences of the Hamiltonian of the active molecule with included the embedding potential yields an approximate, reasonable treatment of embedding effects. The reasons for the effectiveness of these energy expressions lie in the fact that the neglected terms are differences of non-additive energy contributions, which are expected to be small. In contrast, if differential polarization effects are included in terms of state-dependent embedding potentials (responsive environment), we demonstrated that a simple correction must be added to the WF eigenvalue differences of the state-specific Hamiltonians of the embedded molecule, since important Coulomb contributions would otherwise be neglected. The uncorrected calculations with state-specific embedding potentials result in fact in significantly worse excitation energies than with state-independent embedding potentials while the corrected expression may bring the state-specific results into better agreement with the supermolecular reference values. Also in the case of state-dependent embedding potentials, the neglect of non-additive contributions in the evaluation of the excitation energies does not appreciably affect the results.

We demonstrated all these concepts with the state-independent and state-dependent computation of the excitation energies of $p$-nitroaniline, acrolein, methylenecyclopropene, and $p$-nitrophenolate in various solvents. We showed that the use of state-independent potentials generally improves on a QM/MM description. It is also often sufficient to obtain excitation energies in good agreement with the supermolec- 
ular values, surprisingly also in the case of $p$-nitrophenolate in water where calculations with polarizable force fields have instead reported large contributions to the energies from the polarization of the solvent upon excitation of the molecule. For $p$-nitroaniline, we found instead that mutual polarization effects are important and that the use of state-specific embedding can improve the description of the excitation energies of this system.

Finally, when using state-dependent potentials, we found that a key ingredient is the choice of excited-state density for the active part, which we employ to polarize the other fragments and construct a state-specific embedding potential for the WF excited-state calculation. We have outlined ways to obtain approximate excitedstate densities in terms of embedded KS-DFT calculations (also with non-Aufbau occupation numbers) but, when these approximate densities result in rather different excitation energies as in the case of methylenecyclopropene and $p$-nitrophenolate, we take this as indicating that a better description of the excited-state density is needed, possibly through a WF calculation. This particular issue will require further investigation and will be the subject of future studies. 


\section{Appendix: State-average versus state-specific CASSCF and CASPT2 densities}

In Figure 5 of the main chapter, we have computed the ground- and excited-state reference densities of MCP through CASPT2 calculations on top of state-specific (and not state-average) CASSCF calculations, which separately target the ground and excited states. To explain the reasons for this choice, we compare here CASSCF and CASPT2 densities of MCP obtained within state-average and state-specific calculations.

In 3.6, we plot the CASSCF densities and the two CASPT2 densities obtained either as the first-order density $\left(D=D_{0}+D_{1}\right)$ or as the density computed from the first-order wave function, which also contains some second-order contributions $\left(\mathrm{D}=\mathrm{D}_{0}+\mathrm{D}_{1}+\mathrm{D}_{2}^{\prime}\right)$. The symbols $D_{0}, D_{1}$, and $D_{2}^{\prime}$ denote, respectively, the zeroorder CASSCF density, the first-order cross terms between the CASSCF and the first-order contributions in the wave function, and the second-order term given by the expectation value of the density operator on the first-order contributions in the wave function. As in the paper, we analyze the change in linear density between the excited and the ground state given by:

$$
\lambda^{\mathrm{e}}(x)-\lambda^{\mathrm{g}}(x)=\int \rho^{\mathrm{e}}(x, y, z) \mathrm{d} y \mathrm{~d} z-\int \rho^{\mathrm{g}}(x, y, z) \mathrm{d} y \mathrm{~d} z,
$$

where the $\mathrm{x}$-axis is defined by the two carbon atoms in the $\mathrm{CCH}_{2}$ terminus. We observe that both the first-order and the partial second-order density are very different from the zero-order CASSCF one in the case of a state-average calculation, while the corresponding state-specific densities are very close to one another. Furthermore, the large perturbation corrections in the state-average case bring the density in the direction of the state-specific results.

The significant difference between the first-order and the CASSCF density is an indication that the single excitations in the first-order wave function are important (the difference between the first-order and the zero-order density is given by terms which couple the first-order contribution of wave function to the CASSCF one via a one-body operator, so only the single excitations survive in the expectation values). Therefore, in the state-average case, the non-negligible first-order correction to the zero-order density points at the importance of improving on the use of state-average orbitals employed in both the zero-order ground- and excited-state wave functions (the first-order terms can be regarded as an effective orbital-relaxation step). The addition of second-order contributions further modifies the density.

As discussed in the paper, it is a robust procedure to perform state-specific CASSCF calculations for MCP in water since the states are well separated and the wave functions have approximately different symmetry for this nearly planar molecule. The corresponding zero-order, first-order, and partial second-order CASPT2 densities are rather close, which signifies that the zero-order wave functions of the ground and excited states represent a rather good starting point. This must be contrasted to the case of the CASSCF wave functions constructed from state-average orbitals. 


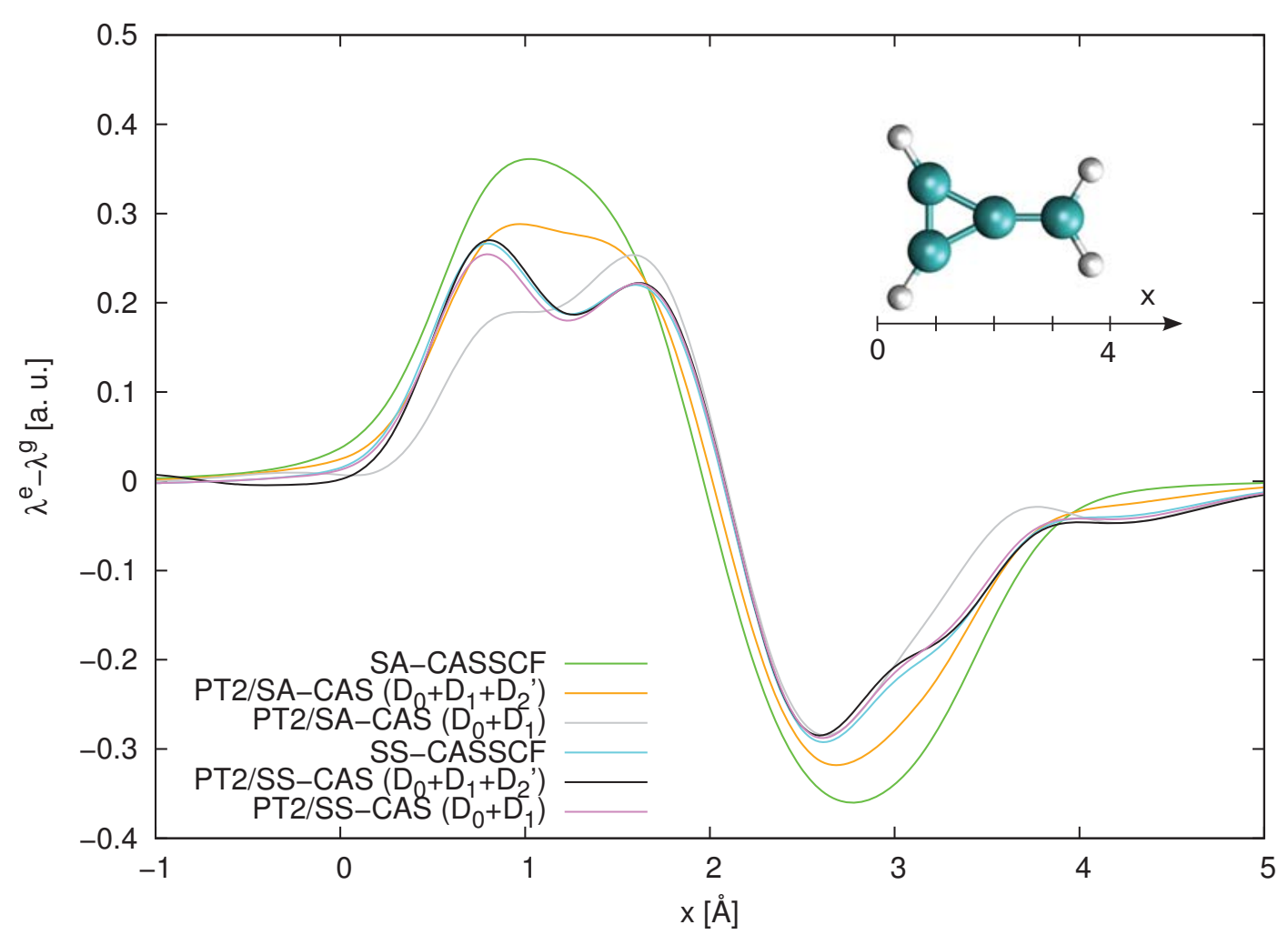

Figure 3.6: Difference between the excited- and ground-state densities of MCP in water integrated over planes perpendicular to the X-axis (Eq. 3.34) and computed with state-specific (SS) and state-averaged (SA) CASSCF and the corresponding CASPT2 calculations.

Therefore, we choose to use the perturbative densities obtained starting from statespecific CASSCF calculations as reference for our subsystem DFT densities. 


\section{Bibliography}

[1] H. M. Senn and W. Thiel, Angew. Chem., Int. Ed. 48, 1198 (2009).

[2] M. Svensson, S. Humbel, R. D. J. Froese, T. Matsubara, S. Sieber, and K. Morokuma, J. Phys. Chem. 100, 19357 (1996).

[3] D. G. Fedorov and K. Kitaura, J. Phys. Chem. A 111, 6904 (2007).

[4] M. S. Gordon, D. G. Fedorov, S. R. Pruitt, and L. V. Slipchenko, Chem. Rev. 112, 632 (2012).

[5] J. Neugebauer, Phys. Reports 489, 1 (2010).

[6] A. Dreuw and M. Head-Gordon, Chem. Rev. 105, 4009 (2005).

[7] A. S. P. Gomes and C. R. Jacob, Annu. Rep. Prog. Chem., Sect. C 108, 222 (2012).

[8] L. Jensen, P. T. van Duijnen, and J. G. Snijders, J. Chem. Phys. 119, 3800 (2003).

[9] K. Sneskov, T. Schwabe, O. Christiansen, and J. Kongsted, Phys. Chem. Chem. Phys. 13, 18551 (2011).

[10] F. Lipparini, C. Cappelli, and V. Barone, J. Chem. Theory Comput. 8, 4153 (2012).

[11] G. Senatore and K. R. Subbaswamy, Phys. Rev. B 34, 5754 (1986).

[12] P. Cortona, Phys. Rev. B 44, 8454 (1991).

[13] M. Iannuzzi, B. Kirchner, and J. Hutter, Chem. Phys. Lett. 421, 16 (2006).

[14] T. A. Wesolowski and A. Warshel, J. Phys. Chem. 97, 8050 (1993).

[15] T. A. Wesolowski and J. Weber, Chem. Phys. Lett. 248, 71 (1996).

[16] T. A. Wesolowski, in Computational Chemistry: Reviews of Current Trends, edited by J. Leszczynski (World Scientific, 2006), Vol. 10, pp. 1-82.

[17] M. E. Casida and T. A. Wesolowski, Int. J. Quantum Chem. 96, 577 (2004). 
[18] J. Neugebauer, J. Chem. Phys. 126, 134116 (2007).

[19] J. Neugebauer, C. Curutchet, A. Muños-Losa, and B. Mennucci, J. Chem. Theory Comput. 6, 1843 (2010).

[20] N. Govind, Y. A. Wang, A. J. R. da Silva, and E. A. Carter, Chem. Phys. Lett. 295, 129 (1998).

[21] N. Govind, Y. A. Wang, and E. A. Carter, J. Chem. Phys. 110, 7677 (1999).

[22] T. A. Wesolowski, Phys. Rev. A 77, 012504 (2008).

[23] P. Huang and E. A. Carter, J. Chem. Phys. 125, 084102 (2006).

[24] O. Roncero, M. P. de Lara-Castells, P. Villarreal, F. Flores, J. Ortega, M. Paniagua, and A. Aguado, J. Chem. Phys. 129, 184104 (2008).

[25] O. Roncero, A. Zanchet, P. Villarreal, and A. Aguado, J. Chem. Phys. 131, 234110 (2009).

[26] C. Huang and E. A. Carter, J. Chem. Phys. 135, 194104 (2011).

[27] F. Aquilante and T. A. Wesolowski, J. Chem. Phys. 135, 084120 (2011).

[28] F. R. Manby, M. Stella, J. D. Goodpaster, and I. Thomas F. Miller, J. Chem. Theory Comput. 8, 2564 (2012).

[29] Since different partitionings of the electron density can be adopted within subsystem density functional theory $[16,65]$, we use the term "polarization" with respect to the situation of no interaction, namely, the isolated case. We note that the notion of polarization is not ambiguous if a unique embedding potential can be achieved for all sub-systems as shown by Elliott et al. [66,67] within DFT-in-DFT, and transferred to WF-in-DFT methods by Huang et al. [26,68].

[30] T. Klüner, N. Govind, Y. A. Wang, and E. A. Carter, Phys. Rev. Lett. 86, 5954 (2001).

[31] T. Klüner, N. Govind, Y. A. Wang, and E. A. Carter, J. Chem. Phys. 116, 42 (2002).

[32] Y. G. Khait and M. R. Hoffmann, J. Chem. Phys. 133, 044107 (2010).

[33] S. Sharifzadeh, P. Huang, and E. A. Carter, J. Phys.: Condens. Matter 21, 355501 (2009).

[34] D. K. Kanan, S. Sharifzadeh, and E. A. Carter, Chem. Phys. Lett. 519-520, 18 (2012).

[35] S. Höfener, A. S. P. Gomes, and L. Visscher, J. Chem. Phys. 136, 044104 (2012). 
[36] We note that the use of an approximate WF method (as opposed to the full configuration interaction limit) does not lead to the ground-state energy of the total system but to an upper bound. Also in case of a frozen environmental density (system 2) which is larger than the exact total density in some region of space, the minimization with respect to the density of system 1 will only lead to an upper bound $[14,22]$. For the calculation of energy differences, this may lead to error cancellation although this is not guaranteed.

[37] A. S. P. Gomes, C. R. Jacob, and L. Visscher, Phys. Chem. Chem. Phys. 10, 5353 (2008).

[38] J. P. Perdew and M. Levy, Phys. Rev. B 31, 6264 (1985).

[39] One can regard this approximation as related to the linearization of the embedding potential, which was shown in Ref. [69] to have only mild effects on orbital energies and other molecular properties in general. An advantage of using the same embedding potential for both electronic states is that the resulting wave functions are orthogonal and transition moments can be evaluated in a straightforward way. Note that this is not the case if the non-linear terms are evaluated exactly, although deviations from orthogonality may be small in practice.

[40] M. J. Frisch, G. W. Trucks, H. B. Schlegel, G. E. Scuseria, M. A. Robb, J. R. Cheeseman, G. Scalmani, V. Barone, B. Mennucci, G. A. Petersson, H. Nakatsuji, M. Caricato, X. Li, H. P. Hratchian, A. F. Izmaylov, J. Bloino, G. Zheng, J. L. Sonnenberg, M. Hada, M. Ehara, K. Toyota, R. Fukuda, J. Hasegawa, M. Ishida, T. Nakajima, Y. Honda, O. Kitao, H. Nakai, T. Vreven, J. J. A. Montgomery, J. E. Peralta, F. Ogliaro, M. Bearpark, J. J. Heyd, E. Brothers, K. N. Kudin, V. N. Staroverov, R. Kobayashi, J. Normand, K. Raghavachari, A. Rendell, J. C. Burant, S. S. Iyengar, J. Tomasi, M. Cossi, N. Rega, J. M. Millam, M. Klene, J. E. Knox, J. B. Cross, V. Bakken, C. Adamo, J. Jaramillo, R. Gomperts, R. E. Stratmann, O. Yazyev, A. J. Austin, R. Cammi, C. Pomelli, J. W. Ochterski, R. L. Martin, K. Morokuma, V. G. Zakrzewski, G. A. Voth, P. Salvador, J. J. Dannenberg, S. Dapprich, A. D. Daniels, Ö. Farkas, J. B. Foresman, J. V. Ortiz, J. Cioslowski, and D. J. Fox, Gaussian 09 Revision A.02, Gaussian Inc. Wallingford CT 2009.

[41] R. Ahlrichs, M. Bär, M. Häser, H. Horn, and C. Kölmel, Chem. Phys. Lett. 162, 165 (1989).

[42] T. H. Dunning Jr., J. Chem. Phys. 90, 1007 (1989).

[43] A. D. Becke, J. Chem. Phys. 98, 5648 (1993).

[44] P. J. Stephens, F. J. Devlin, C. F. Chabalowski, and M. J. Frisch, J. Phys. Chem. 98, 11623 (1994).

[45] A. D. Becke, Phys. Rev. A 38, 3098 (1988). 
[46] C. Lee, W. Yang, and R. G. Parr, Phys. Rev. B 37, 785 (1988).

[47] Amsterdam Density Functional program, Theoretical Chemistry, Vrije Universiteit, Amsterdam, uRL: http://www.scm.com (access date: 15/07/2012).

[48] Y. Zhao and D. G. Truhlar, J. Phys. Chem. A 110, 13126 (2006).

[49] E. V. Lenthe and E. J. Baerends, J. Comput. Chem. 24, 1142 (2003).

[50] A. Lembarki and H. Chermette, Phys. Rev. A 50, 5328 (1994).

[51] T. A. Wesolowski, J. Chem. Phys. 106, 8516 (1997).

[52] S. H. Vosko, L. Wilk, and M. Nusair, Can. J. Phys. 58, 1200 (1980).

[53] F. Aquilante, L. De Vico, N. Ferré, G. Ghigo, P.-Å. Malmqvist, P. Neogrády, T. B. Pedersen, M. Pitoňák, M. Reiher, B. O. Roos, L. Serrano-Andrés, M. Urban, V. Veryazov, and R. Lindh, J. Comput. Chem. 31, 224 (2010).

[54] S. Sharifzadeh, P. Huang, and E. A. Carter, Chem. Phys. Lett. 470, 347 (2009).

[55] G. Ghigo, B. O. Roos, and P.-Å. Malmqvist, Chem. Phys. Lett. 396, 142 (2004).

[56] N. Forsberg and P.-Å. Malmqvist, Chem. Phys. Lett. 274, 196 (1997).

[57] F. Aquilante, P.-Å. Malmqvist, T. B. Pedersen, A. Ghosh, and B. O. Roos, J. Chem. Theory Comput. 4, 694 (2008).

[58] R. A. Kendall, T. H. Dunning Jr., and R. J. Harrison, J. Chem. Phys. 96, 6796 (1992).

[59] W. J. Hehre, R. Ditchfield, and J. A. Pople, J. Chem. Phys. 56, 2257 (1975).

[60] W. L. Jorgensen, J. Chandrasekhar, and J. D. Madura, J. Chem. Phys. 79, 926 (1983).

[61] S. Millefiori, G. Favini, A. Millefiori, and D. Grasso, Spectrochim. Acta A 33, 21 (1977).

[62] S. Kovalenko, R. Schanz, V. Farztdinov, H. Hennig, and N. Ernsting, Chem. Phys. Lett. 323, 312 (2000).

[63] A. F. Moskvin, O. P. Yablonskii, and L. F. Bondar, Theor. Exp. Chem. 2, 469 (1966).

[64] S. W. Staley and T. D. Norden, J. Am. Chem. Soc. 106, 3700 (1984).

[65] A. Savin and T. Wesolowski, in Advances in the Theory of Atomic and Molecular Systems, Vol. 19 of Progress in Theoretical Chemistry and Physics, edited by P. Piecuch, J. Maruani, G. Delgado-Barrio, and S. Wilson (Springer Netherlands, 2009), pp. 311-326. 
[66] P. Elliott, M. H. Cohen, A. Wasserman, and K. Burke, J. Chem. Theory Comput. 5, 827 (2009).

[67] P. Elliott, K. Burke, M. H. Cohen, and A. Wasserman, Phys. Rev. A 82, 024501 (2010).

[68] C. Huang, M. Pavone, and E. A. Carter, J. Chem. Phys. 134, 154110 (2011).

[69] M. Dulak and T. A. Wesolowski, J. Chem. Theory Comput. 2, 1538 (2006). 



\section{Chapter 4}

\section{Wavefunction-in-Density Functional Theory Embedding for Excited States: Which Wavefunctions, which Densities? ${ }^{\dagger}$}

We present a detailed analysis of our recently proposed wavefunction-in-densityfunctional theory method to include differential polarization effects through statespecific embedding potentials. We study methylenecyclopropene and acrolein in water, using several wavefunction (WF) approaches to validate the supermolecular reference and assess their response to embedding. We find that Quantum Monte Carlo, complete-active space second-order perturbation theory, and coupled cluster [CCSD and CCSDR(3)] methods give very consistent solvatochromic shifts and a similar response to embedding. Our scheme corrects the excitation energies produced with a frozen environment, but often overshoots. To ameliorate the problem, one needs to use WF densities to polarize the environment. The choice of the exchange-correlation functional in the construction of the potential has little effect on the excitation, while the approximate kinetic-energy functional appears to be the largest source of error.

\subsection{Introduction}

Density-functional theory (DFT) based subsystem and embedding methods have found widespread applications in the past years (for a recent review, see Ref. [1]) Related are so-called wavefunction-theory-in-DFT (WFT-in-DFT) embedding methods, in which DFT-based embedding potentials are combined with a wavefunction description of an active subsystem. They can be regarded as derived from WFT/DFT hybrid energy expressions [2], or as DFT approaches in which the active subsystem's density is described in terms of a (multi-determinantal) wavefunction. [3] The use of

$\dagger$ This chapter has been published as C. Daday, C. König, J. Neugebauer, and C. Filippi, "Wavefunction-in-Density Functional Theory Embedding for Excited States: Which Wavefunctions, which Densities?", ChemPhysChem 2014, 15, 3205-3217 
such WFT-in-DFT methods for accurate calculations of excitation energies has been pioneered by Carter; [4] a review is provided in Refs. [5] and [6]. A common approximation in such calculations is to simply add an embedding potential for a fixed environmental density to the one-electron terms in the WFT calculation. [7] This potential is derived along the lines of frozen-density embedding, [8] but often additional simplifications are made. An alternative quasi-energy formulation is provided in Ref. [9].

We have recently reported on the first WFT-in-DFT embedding calculations including (approximate) differential polarization effects through state-specific embedding potentials. [10] (See Chapter 3.) "State-specific" here explicitly refers to a freeze-and-thaw-like [11] relaxation of the environmental electron density for the different electronic states of the chromophore. There is also a state-specificity in the embedding potential through its dependence on the electron density of the active system, which we in general expect to be small for weakly overlapping densities (see Chapter 3).

From a formal point of view, state-specific embedding potentials should give an improvement for excitation energies, [12] as they correspond to fully relaxed electron densities in both electronic states. We observed in our study that state-specific embedding potentials can offer an improvement in some cases, but this was not generally true for all systems considered. Hence, the question remains: Which part of our approximate state-specific embedding procedure needs to be improved for more accurate results? Here, we will address two crucial aspects.

First, we will consider other high-level WFT methods for the embedded excitedstate calculation. In Chapter 3, we solely relied on the complete-active-space secondorder perturbation theory (CASPT2) calculations as a reference. However, when comparing environment-induced shifts from CASPT2, the results may actually be biased, because it is often difficult to find a balanced active space and basis set combination for an isolated molecule and the same molecule surrounded by a solvent cluster. So the reference shifts may be affected a bit; but since also differential polarization effects may be comparatively small, it is important to have alternative high-level reference results. In addition, different correlated wavefunction methods may react slightly differently to the embedding potential. Therefore, two different (groups of) WFT methods will be applied in addition to CASPT2 in this work: (i) for the first time, we will employ Quantum Monte Carlo (QMC) techniques in a WFT-inDFT embedding scheme for excited states, since QMC offers an alternative reliable high-accuracy wavefunction description; (ii) we will, in addition, introduce an approximate (linear-response) coupled-cluster-in-DFT embedding scheme that can be used to describe differential polarization effects without including an environmental contribution in the linear-response part. This method thus avoids a major practical bottleneck in applications of more consistent embedded response frameworks [9].

Second, we will compare several (approximate) choices for excited-state densities. These are needed in the relaxation steps to construct the embedding potential for the excited state. The $\triangle \mathrm{SCF}$-like strategy used in our previous chapter will be compared to embedding potentials constructed with excited-state densities from the high-level WFT method. This will be tested here for the case of 
CASPT2. As access to WFT densities is often difficult and considerably increases the computational demands, we also perform a comparison of excited-state densities from two different $\triangle \mathrm{SCF}$-like variants to two types of time-dependent densityfunctional theory (TDDFT) densities: (i) approximate TDDFT densities constructed in a configuration-interaction-singles (CIS) manner, following the approximate interpretation of TDDFT solution vectors suggested by Casida; [13] and (ii) "true" TDDFT densities constructed as a functional derivative of the excited-state energy w.r.t. the external potential. [14] All four different DFT-based excited-state densities will be compared to the CASPT2 density.

In addition, we will test the effect of different exchange-correlation and kineticenergy functionals in the construction of the embedding potentials, and perform an analysis of the resulting embedding potentials. Results from state-specific embedding calculations will be compared to the corresponding state-independent embedding potentials as well as to QM/MM and supermolecular reference calculations.

These are important steps towards an accurate and efficient construction of statespecific embedding potentials for WFT-in-DFT calculations, which pave the way for high-accuracy calculations of excited-state energies for chromophores in environments, such as solvated, biomolecular, or adsorbed dye molecules.

This paper is organized as follows: We discuss aspects regarding our WF-in-DFT embedding method in Section 4.2, and we present the details of our calculations in Section 4.3. Results are given and discussed in Section 4.4 before we conclude our study in Section 4.5.

\subsection{Methods}

In WFT-in-DFT embedding, the total energy of the system is given by the following hybrid expression:

$$
E_{(1+2)}^{\mathrm{WF} / \mathrm{DFT}}=E_{1}^{\mathrm{WF}}\left[\Psi_{1}\right]+E_{2}^{\mathrm{KS}}\left[\rho_{2}\right]+E_{(1 \leftrightarrow 2)}^{\mathrm{OFDFT}}\left[\rho_{1}, \rho_{2}\right]
$$

that is, the sum of the wavefunction energy of subsystem 1 (characterized by $\Psi_{1}$ ), the Kohn-Sham DFT energy of subsystem 2, and an orbital-free-DFT interaction energy between the two. This interaction energy is expressed as:

$$
\begin{aligned}
E_{(1 \leftrightarrow 2)}^{\mathrm{OFDFT}}\left[\rho_{1}, \rho_{2}\right] & =V_{\text {nuc }, 1}\left[\rho_{2}\right]+V_{\text {nuc }, 2}\left[\rho_{1}\right]+J\left[\rho_{1}, \rho_{2}\right] \\
& +E_{x c}^{\text {nadd }}\left[\rho_{1}, \rho_{2}\right]+T_{s}^{\text {nadd }}\left[\rho_{1}, \rho_{2}\right]
\end{aligned}
$$

where $V_{\text {nuc, } I}\left[\rho_{K}\right]$ is the Coulomb attraction between the nuclei of system $I$ and the electron density of system $K, J\left[\rho_{1}, \rho_{2}\right]$ is the Coulomb repulsion between the electron densities of the two systems,

$$
J\left[\rho_{1}, \rho_{2}\right]=\iint \frac{\rho_{1}\left(\mathbf{r}_{1}\right) \rho_{2}\left(\mathbf{r}_{2}\right)}{\left|\mathbf{r}_{1}-\mathbf{r}_{2}\right|} \mathrm{d} \mathbf{r}_{1} \mathrm{~d} \mathbf{r}_{2}
$$


and the non-additive density functionals $F^{\text {nadd }}=\left\{E_{x c}^{\text {nadd }}, T_{s}^{\text {nadd }}\right\}$ are defined as:

$$
F^{\text {nadd }}\left[\rho_{1}, \rho_{2}\right]=F\left[\rho_{1}+\rho_{2}\right]-F\left[\rho_{1}\right]-F\left[\rho_{2}\right],
$$

where a kinetic energy functional must be used to compute the non-additive kinetic terms.

Minimization of the energy expression (Eq. 4.1) with respect to $\Psi_{1}$ leads to a wave function equation containing an additional local potential due to the environment (subsystem 2), which consists of two electrostatic and two non-additive terms:

$$
\begin{aligned}
v_{\mathrm{emb}, 1}(\mathbf{r}) & =v_{\text {nuc }, 2}(\mathbf{r})+\int \frac{\rho_{2}\left(\mathbf{r}^{\prime}\right)}{\left|\mathbf{r}-\mathbf{r}^{\prime}\right|} \mathrm{d} \mathbf{r}^{\prime} \\
& +\underbrace{\frac{\delta E_{x c}^{\text {nadd }}\left[\rho_{1}, \rho_{2}\right]}{\delta \rho_{1}(\mathbf{r})}}_{v_{\mathrm{xc}}\left[\rho_{1}, \rho_{2}\right](\mathbf{r})}+\underbrace{\frac{\delta T_{x c}^{\text {nadd }}\left[\rho_{1}, \rho_{2}\right]}{\delta \rho_{1}(\mathbf{r})}}_{v_{\mathrm{t}}\left[\rho_{1}, \rho_{2}\right](\mathbf{r})} .
\end{aligned}
$$

To somewhat simplify the WFT-in-DFT embedding calculations, we first perform a freeze-and-thaw subsystem DFT calculation, in which systems 1 and 2 are mutually polarized until self-consistency [11], and then employ the resulting embedding potential in the wavefunction calculation for subsystem 1 .

As in our previous chapter we dub scheme (A) the approach where we use the same embedding potential to compute both the ground and the excited states of subsystem 1. The total excitation energy is then obtained as the eigenvalue difference of the embedding Hamiltonian (the Hamiltonian of system 1 with the additional embedding potential) plus a "non-additive" correction term:

$$
\begin{aligned}
\Delta E^{(A)} & =E_{1}^{\mathrm{WF}}\left[\Psi_{1}^{e}\right]+\left\langle\Psi_{1}^{e}\left|\sum_{i} v_{\mathrm{emb}, 1}\left(\mathbf{r}_{i}\right)\right| \Psi_{1}^{e}\right\rangle \\
& -E_{1}^{\mathrm{WF}}\left[\Psi_{1}^{g}\right]-\left\langle\Psi_{1}^{g}\left|\sum_{i} v_{\mathrm{emb}, 1}\left(\mathbf{r}_{i}\right)\right| \Psi_{1}^{g}\right\rangle+\delta E_{\text {nadd }}^{(\mathrm{A})}
\end{aligned}
$$

where

$$
\begin{aligned}
\delta E_{\text {nadd }}^{(\mathrm{A})} & =E_{x c}^{\text {nadd }}\left[\rho_{1}^{e}, \rho_{2}\right]-E_{x c}^{\text {nadd }}\left[\rho_{1}^{g}, \rho_{2}\right] \\
& +T_{s}^{\text {nadd }}\left[\rho_{1}^{e}, \rho_{2}\right]-T_{s}^{\text {nadd }}\left[\rho_{1}^{g}, \rho_{2}\right] \\
& -\int\left\{v_{x c}^{\text {nadd }}\left[\rho_{1}^{g}, \rho_{2}\right](\mathbf{r})+v_{t}^{\text {nadd }}\left[\rho_{1}^{g}, \rho_{2}\right](\mathbf{r})\right\} \\
& \times\left[\rho_{1}^{e}(\mathbf{r})-\rho_{1}^{g}(\mathbf{r})\right] \mathrm{d} \mathbf{r} .
\end{aligned}
$$

This contribution corrects for the fact that the excitation energy should contain the non-additive energy terms (Eq. 4.4) instead of the expectation value of their functional derivatives contained in the embedding potentials. For a detailed derivation of this and the following equations, we refer the reader to Chapter 3.

In scheme (A), the environment does not respond to the electronic excitation of subsystem 1. To account for differential polarization effects, one needs to relax subsystem 2 in the presence of an excited system 1, and iterate. Such a scheme, 
denoted as (B), leads to state-specific embedding potentials, that is, one potential for the ground state as obtained in scheme (A), and a different potential for each excited state of interest. The excitation energy will then be given by the following expression:

$$
\begin{aligned}
\Delta E^{(B)} & =E_{1}^{\mathrm{WF}}\left[\Psi_{1}^{\mathrm{pol}, e}\right]+\left\langle\Psi_{1}^{\mathrm{pol}, e}\left|\sum_{i} v_{\mathrm{emb}, 1}^{\mathrm{pol}, e}\left(\mathbf{r}_{i}\right)\right| \Psi_{1}^{\mathrm{pol}, e}\right\rangle \\
& -E_{1}^{\mathrm{WF}}\left[\Psi_{1}^{g}\right]-\left\langle\Psi_{1}^{g}\left|\sum_{i} v_{\mathrm{emb}, 1}\left(\mathbf{r}_{i}\right)\right| \Psi_{1}^{g}\right\rangle \\
& +\delta E_{\text {nadd }}^{(\mathrm{B})}+\delta E_{\text {simple }}^{(B)}
\end{aligned}
$$

where the two corrections are defined as:

$$
\begin{aligned}
\delta E_{\text {nadd }}^{(\mathrm{B})} & =E_{x c}^{\text {nadd }}\left[\rho_{1}^{\mathrm{pol}, e}, \rho_{2}^{\mathrm{pol}}\right]-E_{x c}^{\mathrm{nadd}}\left[\rho_{1}^{g}, \rho_{2}\right] \\
& +T_{s}^{\text {nadd }}\left[\rho_{1}^{\mathrm{pol}, e}, \rho_{2}^{\mathrm{pol}}\right]-T_{s}^{\text {nadd }}\left[\rho_{1}^{g}, \rho_{2}\right] \\
& -\int\left[v_{x c}^{\text {nadd }}\left[\rho_{1}^{\mathrm{pol}, e}, \rho_{2}^{\mathrm{pol}}\right](\mathbf{r})+v_{t}^{\text {nadd }}\left[\rho_{1}^{\mathrm{pol}, e}, \rho_{2}^{\mathrm{pol}}\right](\mathbf{r})\right] \\
& \times \rho_{1}^{\mathrm{pol}, \mathrm{e}}(\mathbf{r}) \mathrm{d} \mathbf{r} \\
& +\int\left[v_{x c}^{\text {nadd }}\left[\rho_{1}^{g}, \rho_{2}\right](\mathbf{r})+v_{t}^{\text {nadd }}\left[\rho_{1}^{g}, \rho_{2}\right](\mathbf{r})\right] \rho_{1}^{g}(\mathbf{r}) \mathrm{d} \mathbf{r}, \\
\delta E_{\text {simple }}^{(B)} & =E_{2}^{\mathrm{KS}}\left[\rho_{2}^{\mathrm{pol}}\right]-E_{2}^{\mathrm{KS}}\left[\rho_{2}\right] \\
& +\int v_{\text {nuc }, 1}(\mathbf{r}) \Delta \rho_{2}(\mathbf{r}) \mathrm{d} \mathbf{r} .
\end{aligned}
$$

The first correction is analogous to the non-additive contribution in scheme (A) (Eq. 4.7), namely, the difference between the non-additive functionals and the integral of their functional derivatives. The second term, also called the "environmental" correction, includes those energy terms that depend only on $\rho_{2}$ and would therefore vanish if the environment were not relaxed as in scheme (A). The accurate determination of this second correction term is crucial to the success of scheme (B): It is usually of the same order of magnitude as the excitation energy itself (see Chapter 3) and, therefore, even relatively small errors in its value can undermine any improvement scheme (B) can yield on (A). The excitation energy obtained within scheme (B) should in principle be lower than the one determined through scheme (A) since the ground-state WF-in-DFT energy is identical in both cases, while scheme (B) allows more degrees of freedom for the excited state. Therefore, whenever scheme (B) produces a blue shift, this suggests an unresolved issue either with the embedding potential or the environmental correction (the non-additive correction is non problematic as it is typically at least one order of magnitude smaller).

Finally, we note that the environmental correction has a slightly different expression for the case of pseudopotentials as used for instance in the QMC calculations:

$$
\begin{aligned}
\delta E_{\text {simple }}^{(\mathrm{B}), \mathrm{PP}} & =E_{2}^{\mathrm{KS}}\left[\rho_{2}^{\mathrm{pol}}\right]-E_{2}^{\mathrm{KS}}\left[\rho_{2}\right] \\
& +\int v_{\mathrm{PP}, 1}(\mathbf{r}) \Delta \rho_{2}(\mathbf{r}) \mathrm{d} \mathbf{r}
\end{aligned}
$$


where $v_{\mathrm{PP}, 1}(\mathbf{r})$ denotes the sum of the pseudopotentials of the atoms in subsystem 1. Since the ADF code we employ to compute this correction does not allow the use of pseudopotentials, we approximate this integral by simply using the $-Z_{\text {eff }} / r$ component of the pseudopotential. This approximation amounts to the neglect of the non-local components of the pseudopotentials which have however rapid exponential decay. We show below that, for the systems investigated here, this simple procedure for computing the environmental correction reproduces the all-electron excitation energies at the CASPT2 level.

\subsubsection{State-specific CC-in-DFT calculations}

In this paper, we also perform CC-in-DFT calculations including the environmental response not in linear response but, analogously to our CASPT2 calculations, with the use of state-specific embedding potentials and separate calculations for each state. The ground-state energy is then the coupled-cluster result in the ground-state potential, while the excited-state energy is the sum of the ground-state energy and the linear-response excitation both computed in the excited-state potential, but without environmental contributions in the linear response part of the calculation. For the CCSDR(3) calculations, we add ground-state CC 3 energies to the excitation energies computed at the level of $\operatorname{CCSDR}(3)$, since $\operatorname{CCSDR}(3)$ is only a response model. The excitation energies with scheme (B) at the CC2 or CCSD level are therefore given by:

$$
\begin{aligned}
\Delta E_{\mathrm{CCSD} / \mathrm{CC} 2}^{(\mathrm{B})} & =E_{\mathrm{CCSD} / \mathrm{CC} 2}^{0, \mathrm{ex}}+\Delta E_{\mathrm{CCSD} / \mathrm{CC} 2}^{\mathrm{ex}}-E_{\mathrm{CCSD} / \mathrm{CC} 2}^{0, \mathrm{gs}} \\
& +\delta E_{\text {nadd }}^{(\mathrm{B})}+\delta E_{\text {simple }}^{(\mathrm{B})}
\end{aligned}
$$

and in CCSDR(3) by:

$$
\begin{aligned}
\Delta E_{\mathrm{CCSDR}(3)}^{(\mathrm{B})} & =E_{\mathrm{CC} 3}^{0, \mathrm{ex}}+\Delta E_{\mathrm{CCSDR}(3)}^{\mathrm{ex}}-E_{\mathrm{CC} 3}^{0, \mathrm{gs}} \\
& +\delta E_{\text {nadd }}^{(\mathrm{B})}+\delta E_{\text {simple }}^{(\mathrm{B})}
\end{aligned}
$$

where $E^{0, \mathrm{ex}}$ and $E^{0, \mathrm{gs}}$ refer to the ground-state energy in the excited- and the groundstate embedding potentials, respectively.

\subsubsection{Construction of the excited-state density}

We obtain the state-specific embedding potentials from DFT calculations where we use an approximate excited-state density $\rho_{1}$ to generate the corresponding relaxed environmental density $\rho_{2}^{\text {pol,e }}$. In our previous chapter, we considered two different strategies for this, namely, the fully relaxed (fr-KS) and the self-consistent postKohn-Sham (scp-KS) density. In the fr-KS case, we optimize the Kohn-Sham orbitals of system 1 with fixed occupations to represent a singlet excited state characterized by a pure single-orbital transition (a restricted approach with fractional occupation numbers in the frontier orbitals is used here; see Chapter 3 for details). In the scp-KS case, we optimize the Kohn-Sham orbitals for the ground state and only then change 
the occupations to obtain the excited-state density of subsystem 1. Here, we also use a third strategy, namely, to employ wavefunction-based densities. In particular, we use state-specific CASPT2 densities obtained within scheme (A), and relax the environment in the presence of the wavefunction density. For MCP, we verified that further iterations to bring the two subsystems at self-consistency leave the excitation energies unchanged to better than $0.01 \mathrm{eV}$. As discussed in our previous chapter, the use of state-specific perturbation densities for the systems investigated here is appropriate and necessary since the CASPT2 densities obtained from a state-average CASSCF calculation are not sufficiently accurate. We note that, also in the coupled cluster and QMC calculations, we employ the potentials generated with the CASPT2 excited-state densities when comparing the excitation energies computed in the presence of a wave-function-based embedding potential.

For these calculations, we need to compute the wavefunction density with the same basis set as used in the DFT calculations. Therefore, since the CASPT2 densities are obtained in Molcas with a Gaussian basis while the ADF software employs Slater basis sets, we perform a Gaussian fit of Slater orbitals [15] for the exponents of the DZP Slater basis set of ADF, using a contraction of 6 Gaussian primitives for every Slater orbital (SDZP-6G). This basis is then used in the state-specific CASPT2 calculations to generate the excited-state density, which is then imported in ADF to obtain the embedding potential. To test convergence, we also employ a SDZP-5G fit with only 5 Gaussian primitives per Slater orbital, and obtain practically unchanged densities. We use the same SDZP-6G basis set to construct the TDDFT densities in the Gaussian 09 code.

\subsection{Computational details}

We employ the geometries obtained in our previous chapter and optimized within DFT with the BLYP $[16,17]$ functional and the cc-pVDZ basis set $[18]$ using the Gaussian 09 [19] code.

All subsystem DFT calculations are performed with the subsystem-DFT implementation [20,21] in the ADF2013.2 package. [22-24] We employ either the M06-HF [25] or the PW91 [26] exchange-correlation functional and the DZP basis set [27]. For the embedding calculations, we use the Thomas-Fermi (TF) [28-30] or the PW91k [31] kinetic-energy functional to compute the non-additive terms of the kinetic energy. We employ the PW91 functional for the non-additive exchangecorrelation contributions.

For the linear-response coupled-cluster at the approximate doubles (CC2), [3234] singles and doubles (CCSD), [35] and non-iterative triples [CCSDR(3)] [36, 37] orders, we use the Dalton 2013 code [38] appropriately modified for the CC-in-DFT embedding calculations [7]. The CC2 calculations for the complete solvated systems are performed with the Turbomole code, version 6.3.1 [39] and the resolution of the identity [33]. The $1 s$ core orbitals for heavy atoms were kept frozen in these calculations.

We use Molcas 7.4 [40] for the CASPT2 calculations and a modified version 
of the code for the CASPT2-in-DFT embedding calculations, where the embedding interface is adapted from the Molcas-Embed interface developed by Carter and coworkers. [41, 42] We use state-averaged complete-active-space self-consistent field (CASSCF) wave functions with equal weights on the ground and the excited states of interest, and refer the reader to the previous chapter for a description of the choice of active spaces. In the CASPT2 calculations, we employ the default IPEA zero-order Hamiltonian [43] and introduce an additional constant imaginary shift [44] of 0.1 a.u. In the CASPT2 calculations, we freeze the lowest $\sigma$ orbitals corresponding to the $1 \mathrm{~s}$ atomic orbitals of the heavy atoms in the system. We use the Cholesky decomposition of the two-electron integrals [45] with a threshold of $10^{-4}$. All CASPT2 excitation energies reported in this work are obtained at the single-state level.

For the CC and the CASPT2 embedding calculations, we employ the aug-ccpVDZ [18] basis sets for the solute molecule. At variance with Chapter 3, where we treated the water molecules in the supermolecular calculations with the 6-31G basis set, we give here a more balanced description of the solute and the solvent when they are both treated at the WFT level: For solvated methylenecyclopropene (MCP), we use the aug-cc-pVDZ basis set to describe all explicit water molecules and, in the supermolecular calculations for acrolein, we treat the 10 water molecules closest to the formylic oxygen with the aug-cc-pVDZ and the remaining 9 waters with the cc-pVDZ basis set. Within CC2, we can test the use of aug-cc-pVDZ for all 19 surrounding waters and obtain excitation energies for the $n \rightarrow \pi^{*}$ and $\pi \rightarrow \pi^{*}$ states which are compatible within $0.02 \mathrm{eV}$. In order to compare with QMC, we also perform pseudopotential calculations within CASPT2, employing the Stuttgart/Cologne pseudopotentials and basis sets [46] augmented with the diffuse functions from augcc-pVDZ [47].

The program package CHAMP [48] is used for the QMC calculations with scalarrelativistic energy-consistent Hartree-Fock pseudopotentials and the corresponding basis sets. $[49,50]$ In particular, we employ the cc-pVDZ basis augmented with diffuse $s$ and $p$ functions [51] on the heavy atoms. Different Jastrow factors are used to describe the correlation with different atom types and, for each atom type, the Jastrow factor consists of an exponential of the sum of two fifth-order polynomials of the electron-nucleus and the electron-electron distances, respectively [52]. The inclusion of electron-electron-nucleus terms in the Jastrow factor is tested and leads to excitation energies which are equivalent in diffusion Monte Carlo (DMC) within the statistical error. We employ the same CAS spaces used in the CASPT2 calculations and obtain the starting determinantal components of the wavefunction in a CASSCF calculation done with the GAMESS(US) package. [53] In the embedding calculations of MCP, all (linear, Jastrow, and orbital) parameters in the QMC wavefunctions are optimized in a state-average fashion within variational Monte Carlo (VMC) using the linear method [54] extended to state-average calculations. [55] In the cluster calculations, we only optimize the linear and Jastrow parameters and test the effect of also optimizing the orbitals for the isolated and the 3-water system. In both cases, we find that the excitation energies are lowered by about $0.05 \mathrm{eV}$. The pseudopotentials are treated beyond the locality approximation $[56,57]$ and an imaginary time step of 0.05 a.u. is used in the DMC calculations. All QMC results shown below are 
obtained within DMC.

In the QM/MM calculations, we use the TIP3P [58] values of $\mathrm{q}_{\mathrm{O}}=-0.834, \mathrm{q}_{\mathrm{H}}=$ 0.417 when treating the water molecules as static point charges.

\subsection{Results}

We revisit here the performance of the WFT-in-DFT embedding schemes (A) and (B) for the two smallest molecules studied in our previous chapter, namely, methylenecyclopropene (MCP) and s-cis-acrolein (henceforth, acrolein). These molecules are interesting because their low-lying excitations have internal charge-transfer character, and are therefore archetypal examples of strong coupling with the solvent environment. Their small size is also well suited to a detailed investigation with wavefunction approaches of high computational cost.

An important motivation for investigating MCP and acrolein in more detail is also that the performance of scheme (B) is not fully satisfactory for these molecules: The excitation energies of MCP in a responsive water solvent display a strong dependence on the approximate excited-state density chosen to generate the embedding potential and the agreement with the supermolecular results for acrolein is not very good in the case of water solvent (see Chapter 3.

Here, we first establish the reliability of the supermolecular reference used to assess the quality of the embedding calculations, through an extensive comparison of a variety of wavefunction approaches. We then analyze how different exchangecorrelation and kinetic functionals affect the state-independent and state-specific embedding potentials and the resulting excitation energies of solvated MCP and acrolein. We also employ wavefunction densities to polarize the environment in the ground as well as in the excited state, and investigate the effect of partitioning the cluster in subsystems by including an increasing number of water molecules in the wavefunction part.

\subsubsection{Methylenecyclopropene}

We analyze the embedding calculations of the $\pi \rightarrow \pi^{*}$ excitation energy of MCP in water from several perspectives. First, we employ several wavefunction methods in addition to CASPT2, which also used in our previous chapter, and investigate their convergence behavior with increasing cluster size to assess the reliability of the supermolecular reference. Second, we compare these reference excitation energies to the embedding results of schemes (A) and (B) computed with the same wavefunction approach. We then investigate how the errors in the embedding methods depend on the system size (discarding some water molecules) and on the size of the active part treated at the WF level (moving some water molecules from the environment to the active subsystem).

In Table 4.1, we present the excitation energies computed with CASPT2, three different flavors of coupled cluster theory, and QMC for MCP surrounded by an increasing number of water molecules. We incorporate up to a total of 17 water 
molecules. We note that the supermolecular calculation with 17 water molecules is not feasible with CCSD and that MCP with three water molecules is the largest system considered at the CCSDR(3) level.

As shown in Fig. 4.1, the shifts in the excitation energy with increasing number of water molecules are rather consistent between the CASPT2, CCSD, CCSD(3), and QMC methods, supporting the reliability of these approaches in describing the changes in the excitation of MCP due to the water solvent. In particular, the agreement between CASPT2, CCSDR(3), and QMC for isolated MCP and the 3-water cluster is very good and the solvatochromic shift for a 9-water system is very similar at the CASPT2, CCSD, and QMC levels, namely, 0.72, 0.73, and 0.76(2) eV, respectively. Furthermore, even though the supermolecular QMC calculation with 17 waters has a large error bar (to keep the computational cost manageable), the total solvatochromic shift of $0.87(4) \mathrm{eV}$ is compatible with the CASPT2 value of 0.86 $\mathrm{eV}$. This must be contrasted to the use of CC2 which systematically underestimates the solvatochromic shift: CC2 yields a lower solvatochromic shift of $0.64 \mathrm{eV}$ for the 9 -water cluster and of $0.78 \mathrm{eV}$ for the total system. Furthermore, CC2 shows an increasing error compared to its CCSD reference with increasing system size here.

These reference data for the supermolecular case with all water molecules are used to assess the quality of the WF-in-DFT embedding calculations which are also listed in Table 4.1. We consider the embedding schemes (A) and (B), and treat all 17 water molecules within DFT, and MCP with the same wavefunction approaches as used above. We first note that using a ground-state CASPT2 density to polarize the environment in scheme (A) gives excitation energies very similar to the ones obtained with a potential constructed from the ground-state DFT density. As illustrated in Fig. 4.2, the excitation energies obtained in scheme (A) are however consistently blue-shifted by about $0.12 \mathrm{eV}$ with respect to the corresponding reference while method (B) yields red-shifted energies by about $0.1 \mathrm{eV}$. The only exception is again $\mathrm{CC} 2$, which gives an error for scheme (A) almost twice as large and an excitation energy in scheme (B) which agrees with the CC2 supermolecular value. As discussed above, this probably reflects an inaccuracy in the solvatochromic shift for the complete system, which is underestimated by CC2. Surprisingly, an accidental agreement with the reference calculation is obtained within scheme (A) if we do not perform freeze-and-thaw cycles but employ the isolated environmental density to construct the embedding potential. We will see below that this procedure leads to poor results in the case of acrolein.

As shown in Table 4.1, we also test the sensitivity of the embedding calculations to the choice of exchange-correlation functional used to the describe the subsystems in the construction of the embedding potential. We employ two different exchangecorrelation functionals, M06-HF and PW91, and find that the resulting excitation energies in both schemes (A) and (B) are rather insensitive to this ingredient. As previously observed in Chapter 3 and also shown in the table, the (B) excitation energy for MCP displays instead a strong dependence on the approximate excitedstate density used to relax the environment. The excitation energy obtained using a scp-KS excited-state density is significantly red-shifted with respect to the value obtained with a fr-KS density. To overcome the limitations of a $\triangle \mathrm{SCF}$-like density, 


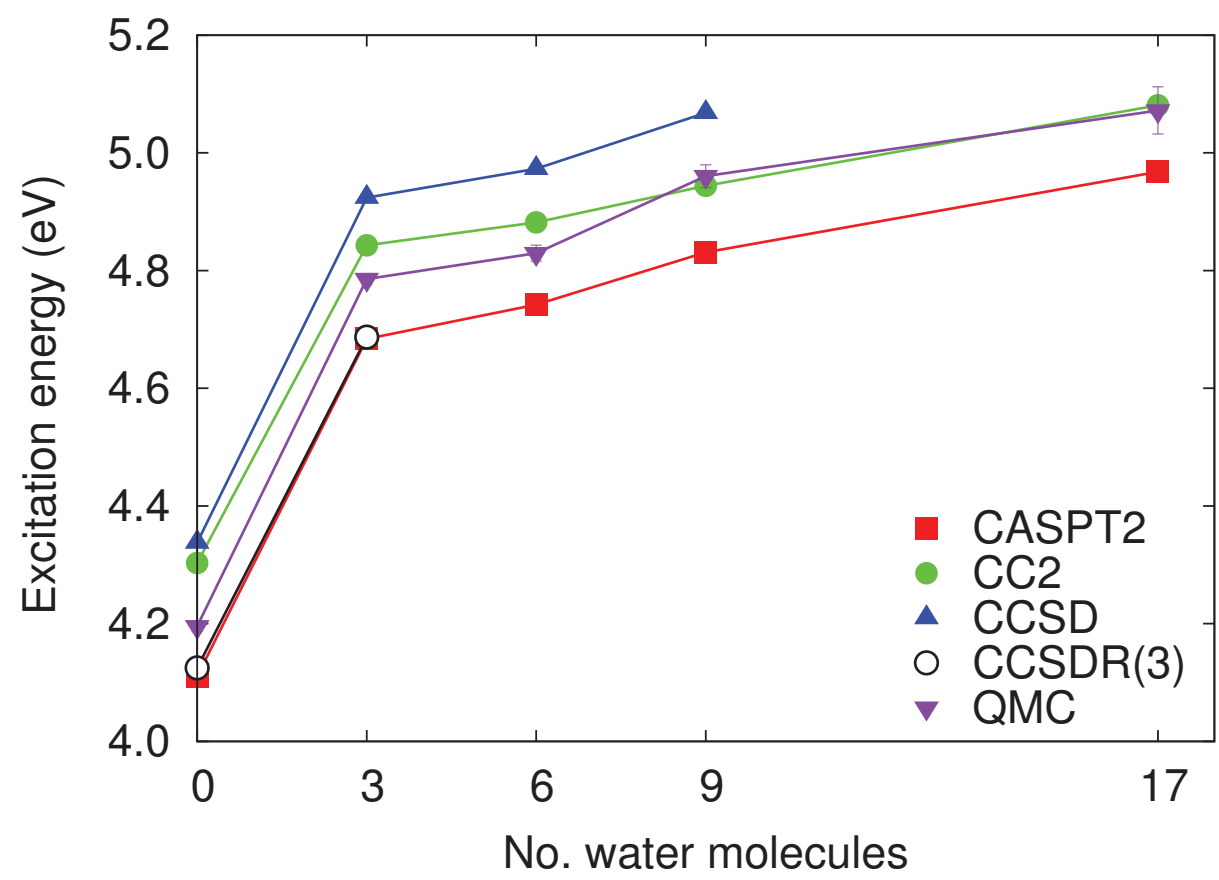

Figure 4.1: Excitation energies (eV) of $\mathrm{MCP}$ and an increasing number of water molecules. We incorporate up to a total of 17 water molecules.

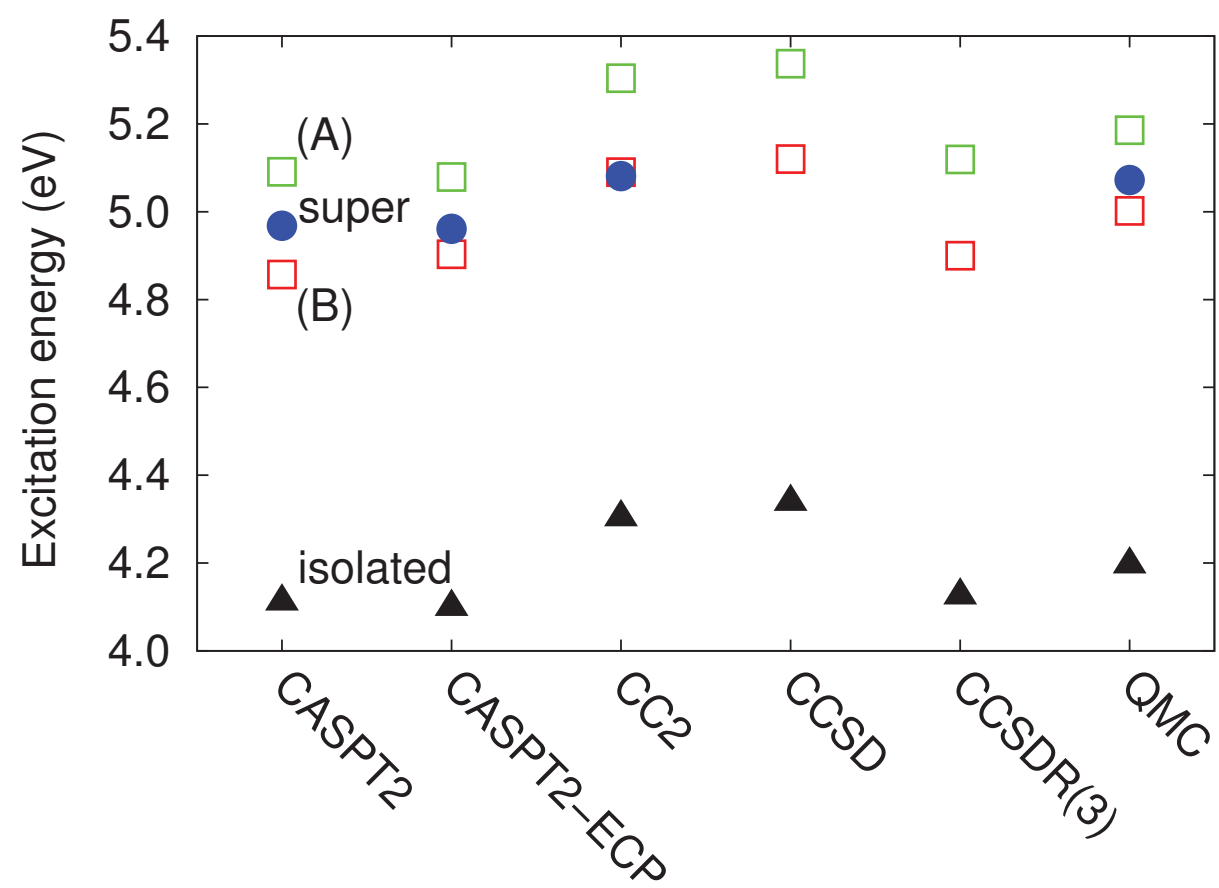

Figure 4.2: Excitation energies (eV) of MCP in the complete water system computed with the WF-in-DFT schemes (A) and (B) and in a supermolecular calculation at the CASPT2 level. The excitation energies for isolated MCP are also shown. The PW91/PW91/PW91k functionals are employed in the embedding calculations and the fr-KS density in scheme (B). 


\begin{tabular}{lcccccc}
\hline \multirow{2}{*}{ \#wat } & \multicolumn{2}{c}{ CASPT2 } & CC2 & CCSD & CCSDR(3) & QMC \\
\cline { 2 - 5 } & AE & ECP & & & & ECP \\
\hline 0 & 4.111 & 4.099 & 4.303 & 4.338 & 4.125 & $4.20(1)$ \\
3 & 4.684 & 4.676 & 4.843 & 4.924 & 4.687 & $4.79(1)$ \\
6 & 4.742 & 4.733 & 4.882 & 4.973 & - & $4.83(1)$ \\
9 & 4.831 & 4.823 & 4.944 & $5.068^{a}$ & - & $4.96(2)$ \\
17 & 4.968 & 4.961 & 5.081 & - & - & $5.07(4)$ \\
\hline \multicolumn{7}{c}{ M06-HF/PW91/PW91k } \\
(A) & 5.104 & 5.094 & 5.315 & 5.348 & 5.132 & $5.19(1)$ \\
(B) fr-KS & 4.867 & 4.912 & 5.084 & 5.116 & 4.897 & $5.02(1)$ \\
iso env & 4.926 & - & - & - & - & \\
(A) & 5.091 & 5.078 & 5.304 & 5.337 & 5.119 & $5.19(1)$ \\
(A) WF & 5.082 & 5.072 & 5.296 & 5.330 & 5.112 & $5.18(1)$ \\
(B) scp-KS & 4.750 & 4.831 & 4.975 & 5.003 & 4.781 & $4.92(1)$ \\
(B) fr-KS & 4.871 & 4.903 & 5.090 & 5.120 & 4.900 & $5.00(1)$ \\
(B) WF & 4.876 & 4.910 & 5.096 & 5.127 & 4.907 & $5.02(1)$ \\
\hline T The cC-pVDZ
\end{tabular}

${ }^{a}$ The cc-pVDZ basis set is used for the $\mathrm{H}$ atoms.

Table 4.1: CASPT2 (all-electron, AE, and effective core potential, ECP), CC, and QMC excitation energies $(\mathrm{eV})$ of MCP and an increasing number of water molecules. We incorporate up to a total of 17 water molecules. In the WF-in-DFT results with schemes (A) and (B), MCP is treated at the WF level and all 17 water molecules within DFT. In scheme (B), we employ $\triangle$ SCF-like (scp-KS and fr-KS) and wavefunction (CASPT2) densities to polarize the environment and construct the embedding potentials. "Iso env" denotes an embedding scheme where the potential is generated with the frozen ground-state density of the isolated environment. We use PW91 and PW91k as the inter-fragment functional and either M06-HF (M06HF/PW91/PW91k) or PW91 (PW91/PW91/PW91k) for the intra-fragment calculations. Only the linear coefficients and Jastrow parameters are optimized in the QMC calculations for the clusters.

we use ground- and excited-state CASPT2 densities to polarize the water molecules in schemes (A) and (B), respectively. For MCP, we find that the use of a WF density yields similar excitation energies to the one obtained with the fr-KS density, so the source of the remaining error with respect to the supermolecular reference must be sought in some other aspects of the embedding schemes.

To this aim, we study the effect of changing the size of the environment, that is, the number of solvent water molecules. We proceed in two different ways: First, we treat only MCP at the WF level and include a given number of water molecules within DFT, discarding all the others. Second, we move the boundary between the WF and DFT parts of the complete system by including an increasing number of water molecules in the WF part.

The results obtained with a growing number of DFT water molecules in the cluster are presented in Fig. 4.3. The results of scheme (A) are blue-shifted with respect 


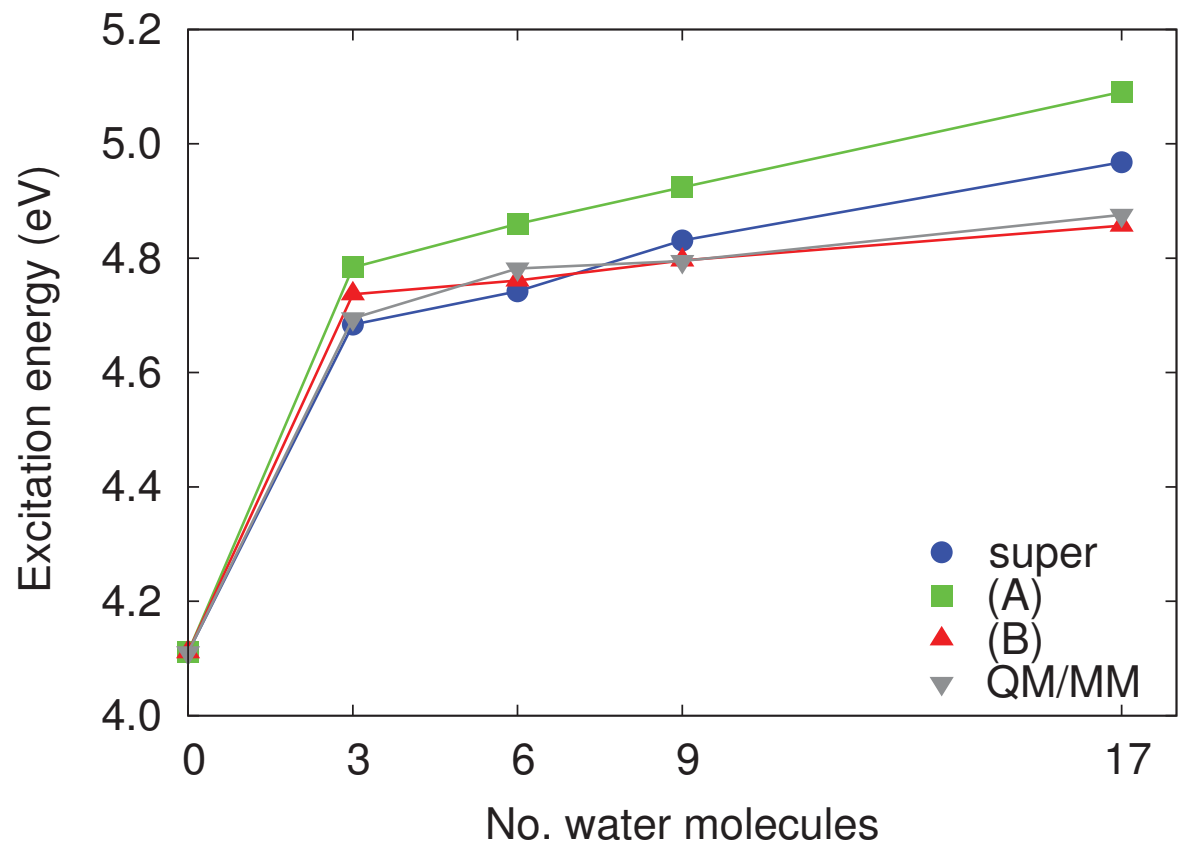

Figure 4.3: CASPT2 excitation energies of MCP and an increasing numbers of water molecules. We incorporate up to a total of 17 water molecules. The corresponding QM/MM and WF-in-DFT energies obtained with schemes (A) and (B) are also shown. The PW91/PW91/PW91k functionals are employed in the embedding calculations and the fr-KS density in scheme (B).

to the energy obtained treating the whole cluster at the WF level, but the size of this shift is quite constant $(0.09-0.12 \mathrm{eV})$ for the $3,6,9$, and 17 water clusters. This suggests that the main source of error of scheme (A) is the neglect of the response of the closest 3 water molecules, while the other 14 water molecules are adequately described as a "frozen" solvent. Scheme (B) correctly yields a red-shift compared to (A) by including the response of the 3 closest water molecules. However, it predicts that this red-shift with respect to scheme (A) grows uniformly with the size of the system to a final value of $0.24 \mathrm{eV}$ for 17 waters, and a resulting excitation energy $0.10 \mathrm{eV}$ lower than the reference value. The environmental response should no doubt increase with the number of solvent molecules but scheme (B) significantly overestimates it since the error with respect to the reference grows with the size of the environment.

In Table 4.2 and Fig. 4.4, we present the excitation energies obtained by including more water molecules in the WF region and treating the remaining ones at the DFT level. We observe that scheme (A) is in very good agreement with the supermolecular reference when we include 3 water molecules in the WF region (blue-shifted by only $0.04 \mathrm{eV}$ for PW91/PW91/PW91k). This finding is very much consistent with the results of the previous test (Fig. 4.3), namely, that the largest error in scheme (A) is the inadequate description of the closest 3 water molecules (possibly, neglecting their response). On the other hand, scheme (B) needs as many as 9 water molecules in the WF region to yield the same level of accuracy. Surprisingly, the use of wavefunction- 


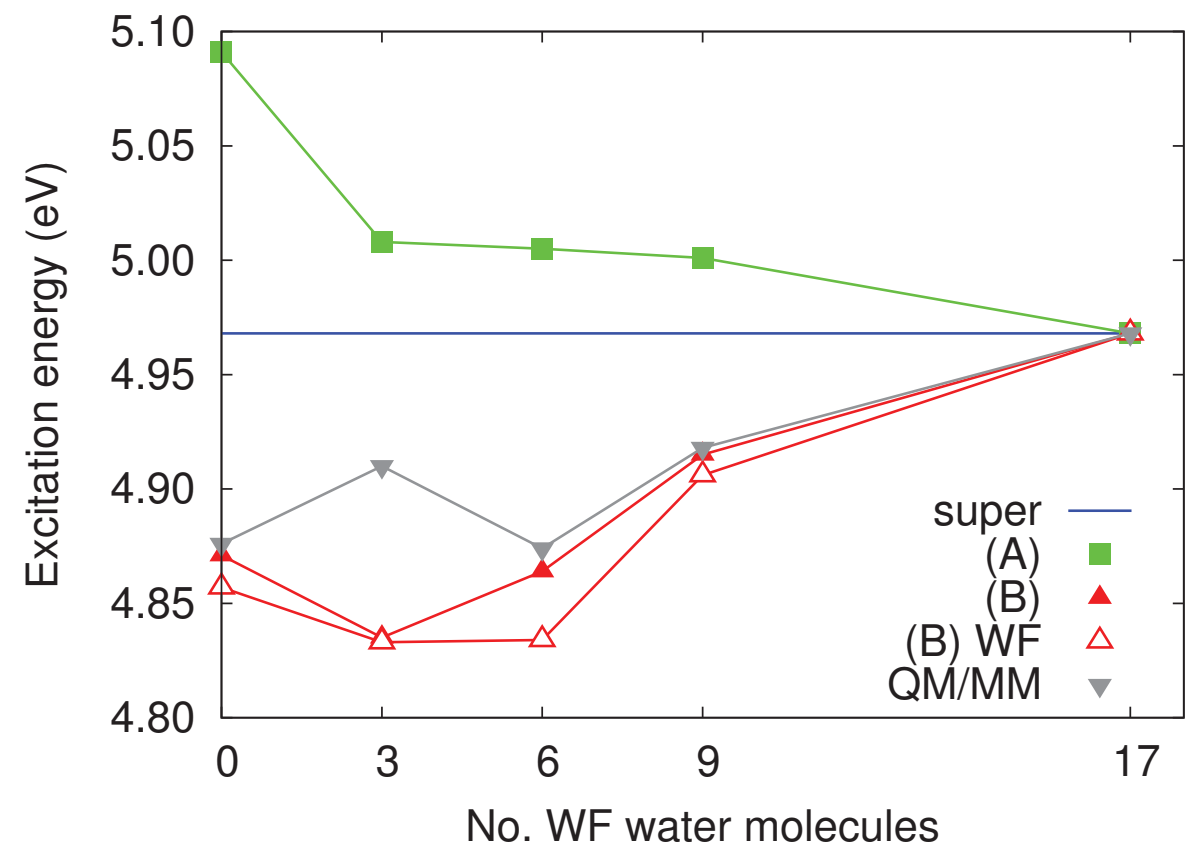

Figure 4.4: Dependence of the CASPT2 excitation energy on the number of water molecules in the WF region. The PW91/PW91/PW91k functionals are employed in the embedding calculations. We use either fr-KS or WF (CASPT2) densities in scheme (B).

based densities brings no significant improvement in the agreement of scheme (B) with the supermolecular value. In fact, for most cases, the QM/MM excitation energy is very close to scheme (B) - this is surely a coincidence as the two approaches are radically different.

We also check for the dependence of the results on the kinetic-energy functional and the exchange-correlation functional used to describe the subsystems. Employing the TF functional instead of PW91k yields a blue shift of 0.02-0.08 eV, depending on the size of the WF region. Therefore, the use of TF as a kinetic-energy functional improves the performance of scheme (B) and worsens the one of scheme (A) when comparing to the reference value. Even though this improvement may be based on error cancellation, it illustrates that the non-additive kinetic-energy functional (and its functional derivative entering the embedding potential) can have a non-negligible effect here, since the desired differential polarization effects are of similar magnitude. M06-HF as an intra-fragment exchange-correlation functional produces slightly less stable results than PW91. For example, there is an unphysical $0.1 \mathrm{eV}$ blue shift of scheme (B) with respect to scheme (A) for the case of 3 water molecules in the WF part. The better performance of PW91 can possibly be explained by the fact that the same density functional is then employed in all aspects of the embedding calculation (intra- and inter-fragment functionals), which translates into a better cancellation of errors.

To better understand why scheme (B) overestimates the red-shift, we also study how the dipole moments of the total system (MCP and solvent) computed with 


\begin{tabular}{|c|c|c|c|c|c|c|c|c|}
\hline \multirow[b]{3}{*}{ \#wat } & \multirow[b]{3}{*}{ QM/MM } & \multirow{2}{*}{\multicolumn{2}{|c|}{$\begin{array}{l}\text { M06-HF } \\
\text { PW91k }\end{array}$}} & \multicolumn{5}{|c|}{ PW91 } \\
\hline & & & & \multicolumn{3}{|c|}{ PW91k } & \multicolumn{2}{|c|}{$\mathrm{TF}$} \\
\hline & & (A) & (B) & (A) & (B) & (B) WF & (A) & (B) \\
\hline 0 & 4.876 & 5.104 & 4.867 & 5.091 & 4.871 & 4.857 & 5.167 & 4.940 \\
\hline 3 & 4.910 & 4.924 & 5.027 & 5.008 & 4.835 & 4.833 & 5.063 & 4.886 \\
\hline 6 & 4.874 & 4.952 & 4.906 & 5.005 & 4.864 & 4.834 & 5.047 & 4.904 \\
\hline 9 & 4.918 & 4.982 & 4.935 & 5.001 & 4.915 & 4.906 & 5.020 & 4.934 \\
\hline 17 & 4.968 & 4.968 & 4.968 & 4.968 & 4.968 & 4.968 & 4.968 & 4.968 \\
\hline
\end{tabular}

Table 4.2: Excitation energies ( $\mathrm{eV}$ ) for MCP computed with different numbers of water molecules in the WF region and the remaining ones treated at the DFT level. In the WF-in-DFT calculations, four different combinations of functionals are employed with M06-HF and PW91 as intra-fragment exchange-correlation functional, and TF and PW91k as kinetic-energy functionals. The non-additive exchange-correlation potential is always computed with the PW91 functional. We use either fr-KS or WF (CASPT2) densities in scheme (B).

schemes (A), (B), and QM/MM compare to the reference values. We treat MCP at the WF level and include only part of the water molecules in the DFT region, discarding the others (as in Fig. 4.3). The dipole contribution of the environment is estimated in schemes (A) with the use of the subsystem DFT density of the water molecules and in QM/MM by simply using the classical electrostatic dipole created by the static point charges. For scheme (B), we compute the dipole contribution of the relaxed environment using the polarized density obtained in a self-consistent fr-KS calculation in the presence of excited MCP.

We plot the difference between the excited- and ground-state dipole moments for our schemes (A), (B), QM/MM, and the supermolecular reference in Fig. 4.5(a). For scheme (A) and QM/MM, the dipole of the environment cancels out but scheme (B) also includes the dipole shift stemming from the environment since the water DFT density is different in the ground and the excited state. The calculations denoted as "(B) only MCP" neglect this change in the environmental density, so only the MCP dipole is included in the calculation. We find that methods (A) and QM/MM seemingly converge to their respective values for the complete system when only 6 water molecules are included. On the other hand, the dipole shift of the supermolecular reference does not converge even for 17 water molecules. In fact, the largest change in the reference dipole shifts occurs between the 9-water cluster and the 17-water one; this observation calls into question the concept that the only effect scheme (A) misses is the response of the nearest 3 water molecules to get an accurate description of the excitation process. However, even though the additional 8 water molecules have apparently a significant response in terms of total density/dipole moment, from our previous analysis, it is clear that the neglect of their response does not amount to a serious error in terms of final excitation energy. We also notice that the agreement of the dipole shift given by scheme (A) with all 17 water molecules and the corresponding supermolecular value is most likely just a coincidence since scheme (A) displays significant errors for all other cluster sizes. 
Scheme (B) shows important similarities to, but also some significant deviations from the supermolecular reference. On the positive side, it is the only embedding scheme tested here which qualitatively correctly predicts a decrease in the dipole shift from 0 to 3 water molecules. However, it overestimates this decrease, and predicts a further decrease for 6 and 9 water molecules instead of an almost constant behavior (in fact, a slight increase) observed in the supermolecular reference. The increase observed for scheme (B) when going from 9 to 17 water molecules is again in very good agreement with the supermolecular result, and much better than for all other methods. If one ignores the environment relaxation, scheme "(B) only MCP" produces larger dipole shifts than (A) or QM/MM. This effect is, however, counterbalanced by the contribution of the responsive DFT environment, which makes the dipole shifts of scheme (B) the smallest of the four schemes considered here. A dipole shift in the active subsystem should indeed induce an opposite (approximately antiparallel) shift in the environment, so scheme (B) works qualitatively as expected, but it clearly overestimates this effect. This finding is also reminiscent of what we have seen in the excitation energies of Fig. 4.3, namely, that scheme (B) overestimates the environmental response.

We also analyze the total ground- and excited-state dipole moments projected to the main axis of MCP in Fig. 4.5(b). As perhaps expected, scheme (A) reproduces the reference dipole moment very well for the ground state (the ground-state dipole moment of scheme (B) is of course identical). Interestingly, scheme (A) is superior to scheme (B) in the excited state even though scheme (A) in no way accounts for the environmental relaxation. Scheme (B) correctly reduces the dipole moment in the excited state with respect to (A) but the correction w.r.t. (A) overshoots the reference value. In fact, it overshoots so much here that a worse agreement with the reference is obtained. Therefore, we witness an over-correction of scheme (B) as in our previous analyses. Finally, we find that QM/MM yields surprisingly good dipoles up to 6 water molecules, especially for the ground state.

\subsubsection{Acrolein}

For acrolein in water, we focus on the comparison of the embedding schemes (A) and (B) with the supermolecular reference, and summarize the results in Table 4.3. We employ CASPT2 and CC2 as wavefunction approaches to describe active acrolein and find that they predict significantly different excitation energies in the presence of the solvent: For the $n \rightarrow \pi^{*}$ and $\pi \rightarrow \pi^{*}$ excitations, CC2 gives supermolecular excitation energies lower than the CASPT2 values by about 0.3 and $0.1 \mathrm{eV}$, respectively. We recall that $\mathrm{CC} 2$ disagrees with CASPT2 also in the case of MCP in water, where higher-level coupled-cluster and QMC approaches have however validated the use of CASPT2 against CC2.

The embedding calculations reproduce the supermolecular reference excitation energy rather well for the $n \rightarrow \pi^{*}$ state. At the CASPT2 level, scheme (A) gives a very good agreement with the reference, independently of the ground-state density (DFT or WF) used to polarize the environment and construct the embedding potential. The excitation energies obtained with scheme (B) depend instead on the choice 

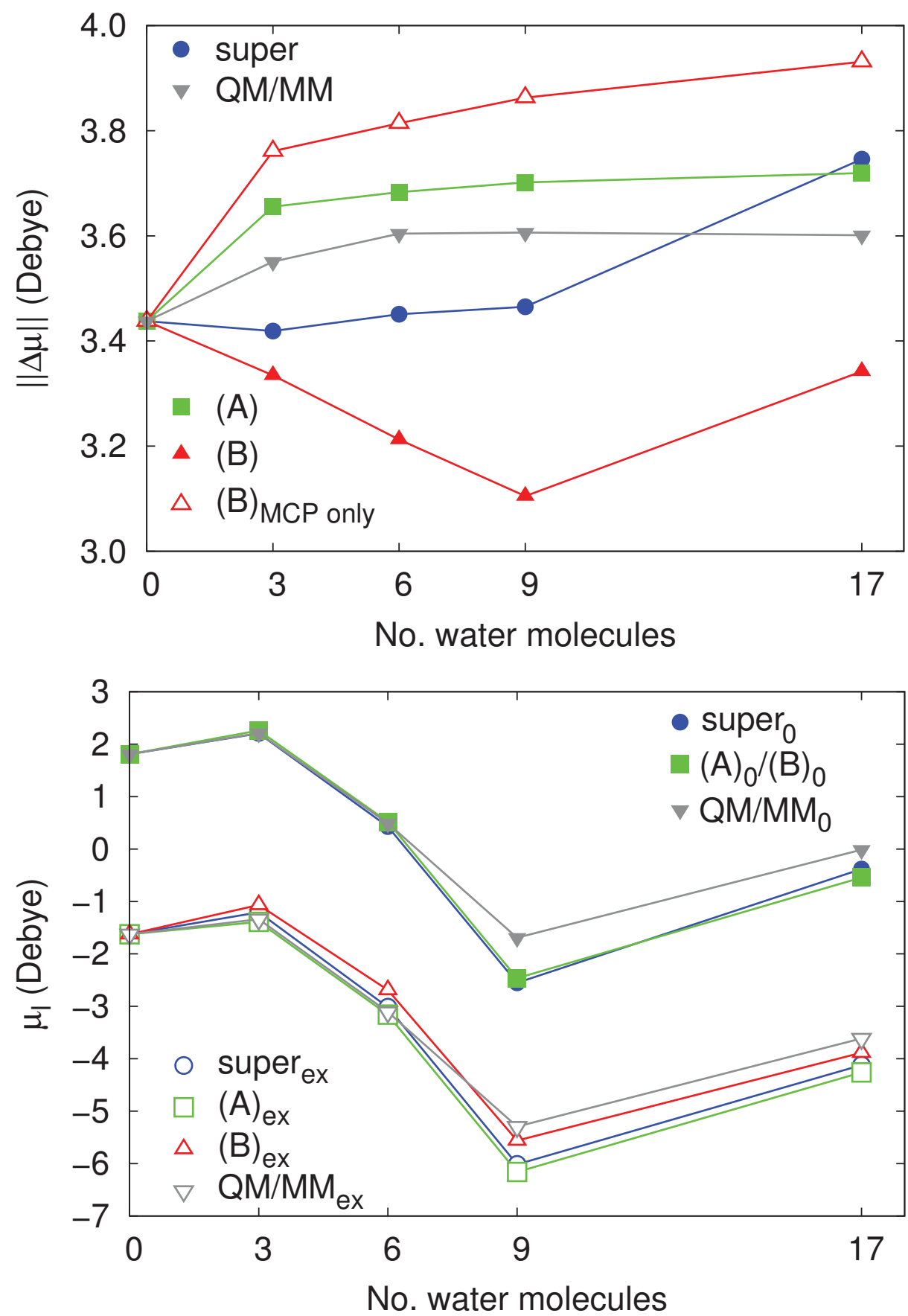

Figure 4.5: Top: Absolute value of the CASPT2 dipole shifts between the groud and excited state. Bottom: ground- and excited-state dipole moments projected on the main axis of MCP. For both cases, we consider MCP and an increasing numbers of water molecules. For schemes (A) and (B), the MCP molecule is treated at the WF level and the water molecules at the DFT level, and the PW91/PW91/PW91k functionals are employed with the fr-KS density in scheme (B). 


\begin{tabular}{lccccccc}
\hline & \multicolumn{3}{c}{$n \rightarrow \pi^{*}$} & & \multicolumn{3}{c}{$\pi \rightarrow \pi^{*}$} \\
\cline { 2 - 4 } \cline { 7 - 8 } scheme & CC2 & CCSD & CASPT2 & & CC2 & CCSD & CASPT2 \\
\hline isolated & 3.280 & 3.371 & 3.289 & & 5.891 & 6.025 & 5.856 \\
iso env & - & - & 4.392 & & - & - & 5.837 \\
(A) & 4.433 & 4.532 & 4.623 & & 5.773 & 5.919 & 5.763 \\
(A) WF & 4.432 & 4.532 & 4.623 & & 5.782 & 5.928 & 5.774 \\
(B) scp-KS & 4.242 & 4.360 & 4.421 & & 5.822 & 5.970 & 5.816 \\
(B) fr-KS & - & - & - & & 5.779 & 5.926 & 5.771 \\
(B) WF & 4.318 & 4.427 & 4.500 & & 5.828 & 5.976 & 5.821 \\
super & 4.340 & - & 4.610 & & 5.580 & - & 5.658 \\
\hline
\end{tabular}

Table 4.3: CASPT2 and CASPT2-in-DFT excitation energies (eV) of acrolein in water. The PW91/PW91/PW91k functionals are employed in the embedding calculations in combination with $\triangle \mathrm{SCF}$ (scp-KS and fr-KS) and WF (CASPT2) densities. We also report the excitation energy of isolated acrolein and the value (iso env) obtained with an embedding scheme where the potential is generated with the frozen ground-state density of the isolated environment.

of the excited-state density used to relax the environment. With the scp-KS density, scheme (B) yields a red-shift of $0.2 \mathrm{eV}$ with respect to the (A) result, overestimating the environmental response and, therefore, spoiling the agreement with the supermolecular calculation. Scheme (B) with the wavefunction-based density fares much better since it predicts a red-shift of only about $0.1 \mathrm{eV}$, which brings the values in better agreement with the supermolecular reference. Interestingly, if we do not perform freeze-and-thaw cycles but employ the isolated environmental density to construct the embedding potential, we obtain a rather poor excitation energy. Therefore, although reasonable for MCP, the procedure of using isolated densities advocated in Ref. [59] is not generally valid and can be rather dangerous for the system at hand if not properly validated.

Unlike the $n \rightarrow \pi^{*}$ state, the $\pi \rightarrow \pi^{*}$ excitation energies obtained with the different embedding schemes and wavefunction methods have a rather small spread (about $0.05 \mathrm{eV}$ ). We note that there is a small, unphysical (see Section 4.2) blue-shift of about $0.04 \mathrm{eV}$ when going from scheme (A) to (B). Finally, we stress once again that the use of $\mathrm{CC} 2$ as supermolecular reference would have in fact lead us to the opposite conclusion as regards the relative accuracy of the (A) and (B) embedding schemes in the case of the $n \rightarrow \pi^{*}$ excitation. This underlines the importance of having an independent reference wavefunction approach, especially in view of the fact that CC2 shifts showed an increasing deviation from their CCSD reference for increasing solvation shell in case of MCP.

Similarly to MCP, the use of a ground-state wavefunction density to polarize the solvent yields practically unchanged excitation energies. This again gives us confidence in the ground-state description of these two systems in WF/DFT. 


\subsubsection{Excited-state densities to polarize the environment}

As discussed above, the accuracy of scheme (B) often hinges on the availability of a good approximation for the excited-state density of the embedded molecule to polarize the environment. The two simple ways (scp-KS and fr-KS) of generating an approximate density within Kohn-Sham DFT can lead to significantly different excitation energies within scheme (B) as observed for MCP, and may be both not sufficiently accurate. Furthermore, it is not always possible to converge the selfconsistent procedure for obtaining the fr-KS density as in the case of the $n \rightarrow \pi^{*}$ state of acrolein.

As demonstrated above, the use of accurate wavefunction-based densities can improve the results obtained with $\triangle \mathrm{SCF}$-like densities or be used to confirm the excitation energies obtained with these densities. Here, we used CASPT2 densities obtained in state-specific calculations since the use of state-average orbitals significantly impacts the quality of the resulting PT2 density (see the appendix of Chapter 3). However, this recipe is not generally applicable since a state-specific calculation can be unsuitable for the system at hand. Moreover, in practical computations of larger systems, it might simply be too costly to determine an accurate density with a wavefunction approach, in particular if iterative relaxation cycles ("freezeand-thaw") are carried out.

An alternative we have not considered so far is to obtain an excited-state density through time-dependent density functional theory (TDDFT). To investigate whether this is a viable strategy, we consider the isolated MCP molecule (TDDFT densities are not yet available for embedding calculations with the ADF code) and compute the linear density difference between the ground and the excited state of MCP defined as:

$$
\lambda^{\mathrm{e}}(x)-\lambda^{\mathrm{g}}(x)=\int \rho^{\mathrm{e}}(x, y, z) \mathrm{d} y \mathrm{~d} z-\int \rho^{\mathrm{g}}(x, y, z) \mathrm{d} y \mathrm{~d} z,
$$

where the $\mathrm{x}$-axis is parallel to the two carbon atoms in the $\mathrm{CCH}_{2}$ terminus. In Fig. 4.6, we compare the results obtained with the reference CASPT2, the two $\triangle \mathrm{SCF}-$ based densities, and two TDDFT densities obtained with the CAM-B3LYP [60] functional. An approximate TDDFT density is computed in a CIS-like fashion [13] and the "exact" linear-response (LR) one is calculated as the functional derivative of the energy with respect to the potential. [14] We find that the scp-KS and CIS-like TDDFT densities are very close to each other and severely overestimate the charge transfer effect while fr-KS slightly underestimates it. As compared to the fr-KS density, the linear-response or "exact" TDDFT density displays a better agreement with the reference on the side of the $\mathrm{CCH}_{2}$ terminus but a larger discrepancy in the ring. We recall that, for MCP, the use of the reference WF density yields very similar excitation energies within scheme (B) to the ones obtained with the fr-KS density while the scp-KS construction leads to severely underestimated energies. Therefore, while CIS-only TDDFT does not bring an improvement compared to a simple scpKS density, the use of the linear-response TDDFT density in scheme (B) represents a promising compromise between accuracy and computational cost. 


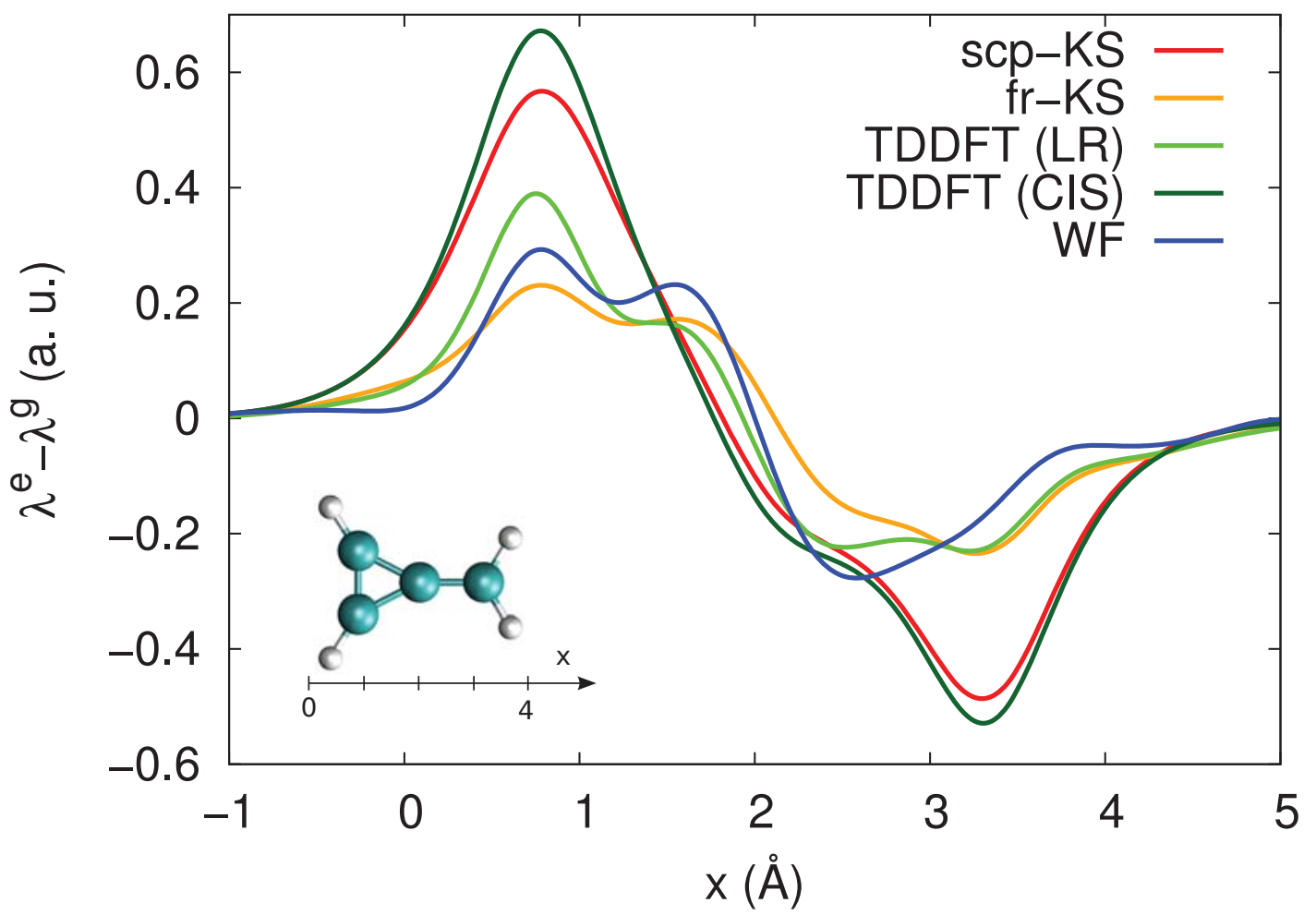

Figure 4.6: Comparison of linear difference densities obtained by DFT/M06-HF (scp-KS and fr-KS), TDDFT/CAM-B3LYP (linear-response and CIS-like), and wave function (CASPT2) methods. Inset: the position of the MCP molecule. 


\subsubsection{On the accuracy of the embedding potentials}

To understand the effect of using state-specific potentials in scheme (B), it is instructive to analyze the embedding potentials. For MCP, we know that scheme (B) yields a red-shifted excitation with respect to scheme (A) and this feature should be reflected in the ground- and excited-state embedding potentials. If we plot the difference between the excited- and ground-state embedding potentials in Fig. 4.7, we observe in fact that the excited-state potential promotes the charge transfer from the methylene moiety to the ring by increasing the potential around the region which loses charge. Therefore, the excited-state potential has the appropriate features to give a qualitatively correct chemical behavior in the excitation.

However, if we want to investigate in more detail how the various ingredients in the calculations affect the embedding potentials, it is difficult to do so through a direct visualization of the potential since MCP is not a linear molecule. Therefore, we choose here to look at the gross features of the potentials by averaging it over an isosurface "outside" the molecule characterized by the same distance to the closest carbon atom of the MCP molecule. In Fig. 4.8, we show the average embedding potential as a function of this coordinate for schemes (A) and (B), which form a very high barrier in the range of about 2-4 $\AA$. As documented in the literature for smaller molecules [61], this barrier is introduced by the non-additive contribution of the functional derivative of the kinetic functional and, while it correctly serves the purpose of confining the electrons in the respective subsystems, it is usually overestimated by present approximate kinetic-energy functionals. Since scheme (B) red-shifts the excited-state energy with respect to scheme (A), one might expect some difference in the average features of the potential or in the height of the barriers. Surprisingly, the average embedding potentials from schemes (A) and (B) and, consequently, the corresponding barriers are practically identical. Therefore, the way in which a statespecific potential affects the excited state at the level of a $0.1-0.2 \mathrm{eV}$ is hidden in the finer features of the potentials and it will be possibly harder to capture when developing newer kinetic-energy functionals. On the other hand, the use of different kineticenergy functionals, PW91k and Thomas-Fermi, in the construction of the potential for scheme (A) yields some clear differences: The barrier formed by Thomas-Fermi is slightly higher than the one obtained with PW91k and the potential raises more steeply. This behavior correlates rather well with the excitation energies, which are consistently blue shifted when the Thomas-Fermi approximation is employed (see Table 4.2). An analogous analysis for acrolein in water yields similar global features in the embedding potential as illustrated in Figure 4.9.

\subsection{Conclusions}

We performed here a detailed analysis of the WF-in-DFT approach we recently proposed for excited-state calculations, which includes differential polarization effects through state-specific embedding potentials. We focused on two molecules (micro)solvated in water, MCP and acrolein, where the use of state-specific embedding 


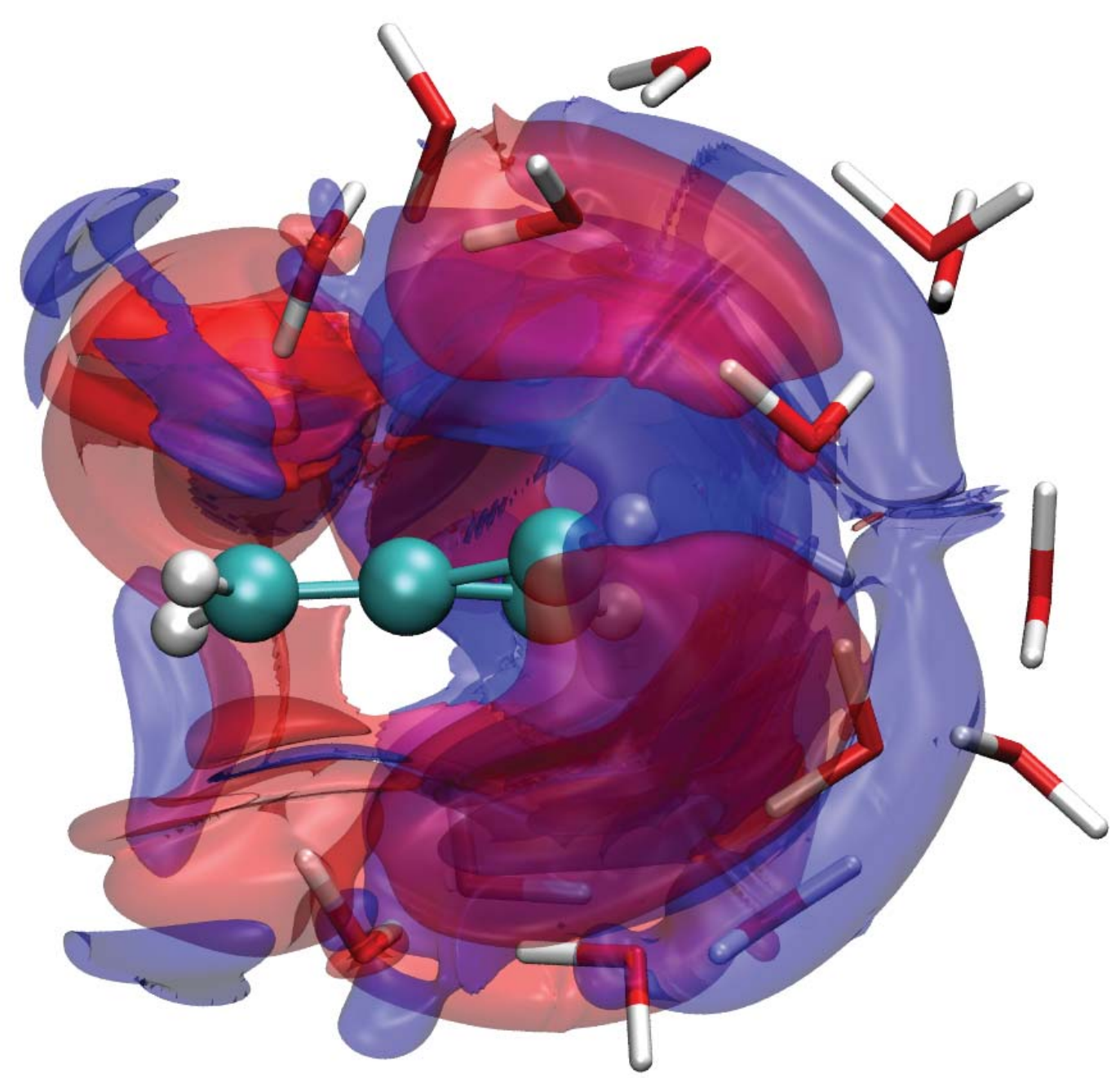

Figure 4.7: The difference between the excited- and ground-state embedding potentials for MCP obtained with the PW91/PW91/PW91k functionals and the fr-KS density in scheme (B). The red and blue isosurfaces correspond to the values of 0.0075 and -0.0075 a.u., respectively. 

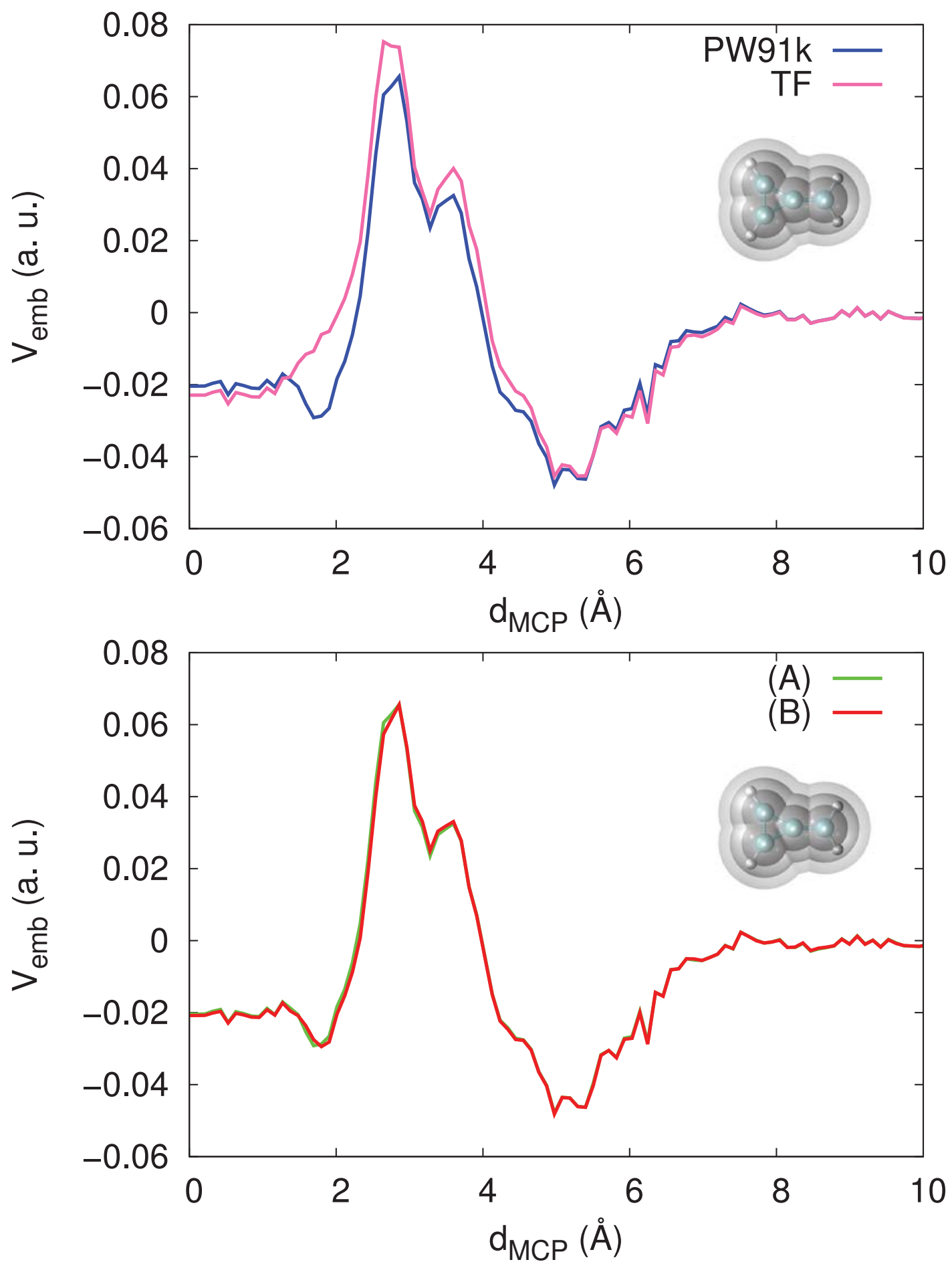

Figure 4.8: Average embedding potential as a function of the distance from the closest carbon atom of MCP. Top: Two different kinetic-energy functionals are used in the construction of the non-additive kinetic potential in scheme (A). Bottom: The potentials are obtained with schemes (A) and (B). PW91 is always employed for the intra-fragment computations and the non-additive exchange-correlation potentials. Inset: Points in space for $\mathrm{d}_{\mathrm{MCP}}=1.0,1.5$, and $2.0 \AA$. 

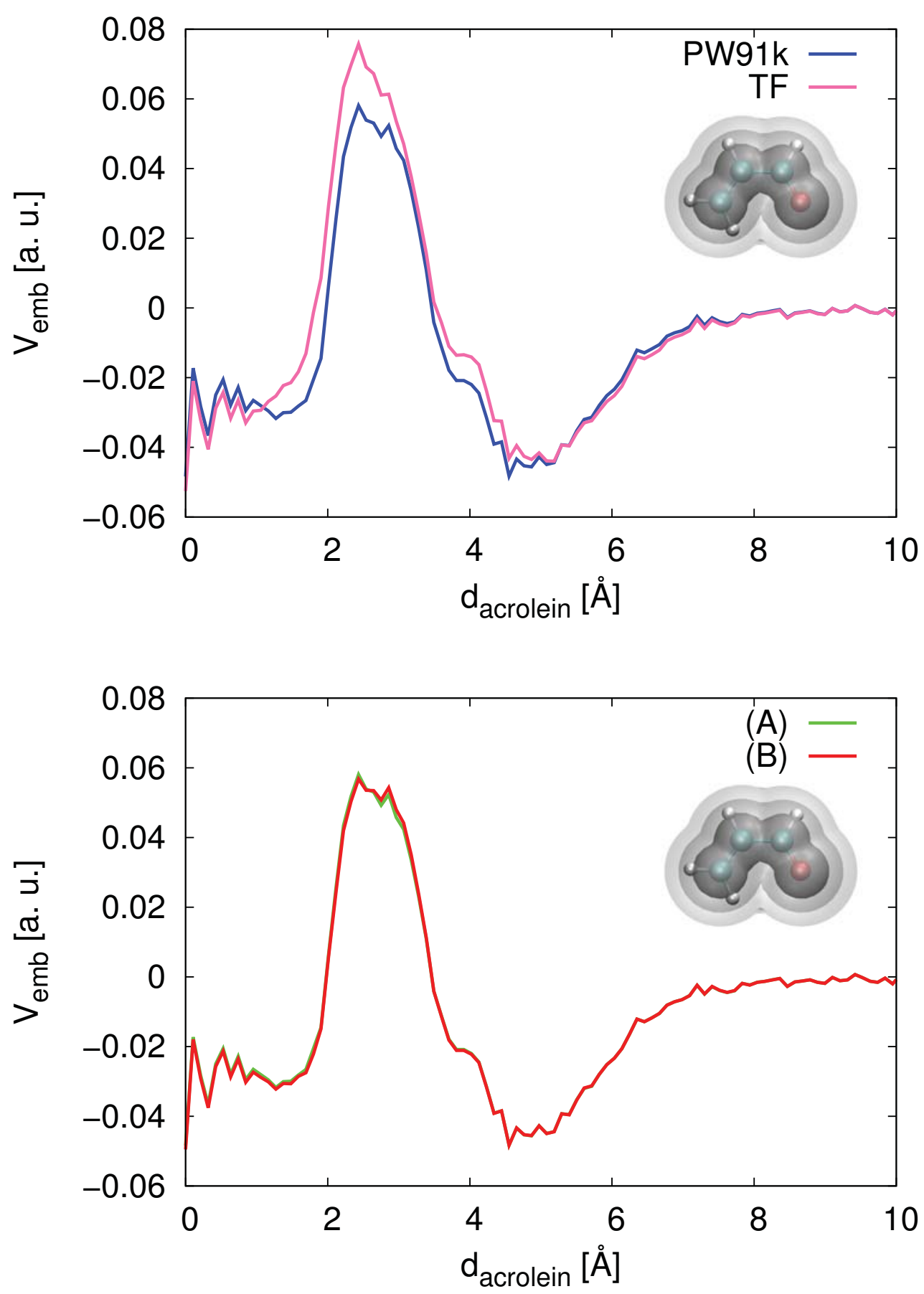

Figure 4.9: Average embedding potential as a function of the distance from the closest heavy atom of acrolein. Top: Two different kinetic-energy functionals are used in the construction of the non-additive kinetic potential in scheme (A). Bottom: The potentials are obtained with schemes (A) and (B). PW91 is always employed for the intra-fragment computations and the non-additive exchange-correlation potentials. Inset: Points in space for $\mathrm{d}_{\text {acro }}=1.0,1.5$, and $2.0 \AA$. 
potentials in Chapter 3 did not improve upon the excitation energies obtained with the standard WF-in-DFT approach with state-independent potentials and a frozen ground-state DFT environment.

First of all, we showed that the supermolecular solvatochromic shifts from CASPT2 calculations, which were exclusively used as a reference in our previous chapter, are confirmed by QMC and by CC methods. In particular, the QMC, CCSD, and CCSDR(3) approaches offer a very similar response to the solvent as more water molecules are added to the system, in good agreement with the CASPT2 method. We note in passing that the $\mathrm{CC} 2$ model in our MCP test case turned out to yield increasing deviations from CCSD for excitation energies with increasing number of solvent molecules. Using the $\mathrm{CC} 2$ shifts as a reference can actually change the conclusion on the relative accuracy of the embedding methods (A) versus (B), as is the case here of the $n \rightarrow \pi^{*}$ excitation of acrolein. Concerning the embedding effect on different electronic-structure methods, we found for MCP that all wavefunction approaches respond to both embedding schemes in a very similar manner: Scheme (A) uniformly leads to a solvatochromic shift of about $1 \mathrm{eV}$, whereas scheme (B) yields smaller shifts of about $0.8 \mathrm{eV}$. For this molecule, scheme (B) leads to slightly better shifts than scheme (A) with the settings tested here (unless "scp-KS" densities are used). Our results demonstrate the importance of using a well validated reference (computed here with a consistently large basis set for the complete system) and an accurate excited-state density in the construction of the embedding potential in scheme (B).

We also investigated in detail how various computational ingredients affect the ground- and excited-state potentials of MCP and acrolein and the resulting excitation energies in scheme (A) and (B). We found that the specific exchange-correlation functional used to describe the subsystems does not significantly affect the excitation energies. However, the choice of the excited-state density used to polarize the environment and to construct the excited-state potential in scheme (B) can play a major role. In order to overcome the limitation of $\triangle \mathrm{SCF}$-like (scp-KS and fr-KS) densities as used in our previous chapter, we tested accurate CASPT2 excited-state densities obtained in scheme (A) to polarize the environmental density. This procedure is computationally more costly, but offers a more consistent WFT-in-DFT embedding approach. Moreover, wavefunction-based densities can be obtained for any state of interest unlike the fr-KS one, for which no convergence could be reached for the $n \rightarrow \pi^{*}$ state of acrolein. We also tentatively analyzed whether TDDFT-based densities represent a viable route with reduced computational cost and, for MCP, observed a very promising agreement of the linear-response TDDFT density with the accurate wavefunction-based reference. This option will have to be further explored and applied to the construction of the excited-state potential for scheme (B).

Even with the use of wavefunction-based excited-state densities in the construction of the excited-state potentials, the results of the WFT-in-DFT embedding with state-specific potentials are not systematically better than scheme (A), which makes use of state-independent embedding potentials. Our analysis shows that scheme (B) qualitatively corrects results from scheme (A) into the right direction, but often leads to an overcorrection. In the case of MCP, state-specific embedding potentials slightly overestimate the response of the environment and therefore also the solvatochromic 
shift: Compared to the supermolecular reference, the errors produced by scheme (B) are similar in magnitude to those of scheme (A), but in the opposite direction. For the $n \rightarrow \pi^{*}$ state of acrolein, we found that scheme (B) again overestimates the environmental response and red-shifts the excitation energy below the reference value. We stress that these errors are rather small, typically below $0.1 \mathrm{eV}$, and that a clear signature of possible inaccuracies in scheme (B) is in fact the dependence of the excitation energy on the excited-state density used to polarize the environment.

Possibly, the presence of such strong dependence on the excited-state density of the active subsystem signals another limitation of the approach, namely, the use of ground-state energy functionals and their functional derivatives to generate excitedstate embedding potentials. At present, theoretical effort on excited-state energy functionals is, however, still mainly focused on developing a proper theoretical formulation $[62,63]$ more than on generating approximations for practical use.

Another source of error which affects both embedding schemes (A) and (B) is of course the relative crudeness of the available approximations for the kinetic energy functional entering in the embedding potentials as compared to the exchangecorrelation counterpart. This was shown here by a comparison of TF and PW91k for the excitation energies of MCP. To understand possible routes of improvement for either scheme, we believe that the comparison with exact reconstructed [61] stateindependent and state-specific embedding potentials is essential. This will be the subject of future investigations. 


\section{Bibliography}

[1] C. R. Jacob and J. Neugebauer, WIREs Comput. Mol. Sci. 4, 325 (2014).

[2] N. Govind, Y. A. Wang, A. J. R. da Silva, and E. A. Carter, Chem. Phys. Lett. 295, 129 (1998).

[3] T. A. Wesolowski, Phys. Rev. A 77, 012504 (2008).

[4] T. Klüner, N. Govind, Y. A. Wang, and E. A. Carter, Phys. Rev. Lett. 86, 5954 (2001).

[5] A. S. P. Gomes and Ch. R. Jacob, Annu. Rep. Prog. Chem. C 108, 222 (2012).

[6] F. Libisch, C. Huang, and E. A. Carter, Acc. Chem. Res. (2014).

[7] A. S. P. Gomes, C. R. Jacob, and L. Visscher, Phys. Chem. Chem. Phys. 10, 5353 (2008).

[8] T. A. Wesolowski and A. Warshel, J. Phys. Chem. 97, 8050 (1993).

[9] S. Höfener, A. S. P. Gomes, and L. Visscher, J. Chem. Phys. 136, 044104 (2012).

[10] C. Daday, C. König, O. Valsson, J. Neugebauer, and C. Filippi, J. Chem. Theory Comput. 9, 2355 (2013).

[11] T. A. Wesolowski and J. Weber, Chem. Phys. Lett. 248, 71 (1996).

[12] Y. G. Khait and M. R. Hoffmann, J. Chem. Phys. 133, 044107 (2010).

[13] M. E. Casida, in Recent Advances in Density Functional Methods Part I, edited by D. P. Chong (World Scientific, 1995), pp. 155-192.

[14] D. Marx and J. Hutter, Ab Initio Molecular Dynamics - Basic Theory and Advanced Methods (Cambridge University Press, 2009).

[15] W. J. Hehre, R. F. Stewart, and J. A. Pople, J. Chem. Phys. 51, 2657 (1969).

[16] A. D. Becke, Phys. Rev. A 38, 3098 (1988).

[17] C. Lee, W. Yang, and R. G. Parr, Phys. Rev. B 37, 785 (1988). 
[18] T. H. Dunning Jr., J. Chem. Phys. 90, 1007 (1989).

[19] M. J. Frisch, G. W. Trucks, H. B. Schlegel, G. E. Scuseria, M. A. Robb, J. R. Cheeseman, G. Scalmani, V. Barone, B. Mennucci, G. A. Petersson, H. Nakatsuji, M. Caricato, X. Li, H. P. Hratchian, A. F. Izmaylov, J. Bloino, G. Zheng, J. L. Sonnenberg, M. Hada, M. Ehara, K. Toyota, R. Fukuda, J. Hasegawa, M. Ishida, T. Nakajima, Y. Honda, O. Kitao, H. Nakai, T. Vreven, J. J. A. Montgomery, J. E. Peralta, F. Ogliaro, M. Bearpark, J. J. Heyd, E. Brothers, K. N. Kudin, V. N. Staroverov, R. Kobayashi, J. Normand, K. Raghavachari, A. Rendell, J. C. Burant, S. S. Iyengar, J. Tomasi, M. Cossi, N. Rega, J. M. Millam, M. Klene, J. E. Knox, J. B. Cross, V. Bakken, C. Adamo, J. Jaramillo, R. Gomperts, R. E. Stratmann, O. Yazyev, A. J. Austin, R. Cammi, C. Pomelli, J. W. Ochterski, R. L. Martin, K. Morokuma, V. G. Zakrzewski, G. A. Voth, P. Salvador, J. J. Dannenberg, S. Dapprich, A. D. Daniels, Ö. Farkas, J. B. Foresman, J. V. Ortiz, J. Cioslowski, and D. J. Fox, Gaussian 09 Revision A.02, Gaussian Inc. Wallingford CT 2009.

[20] J. Neugebauer, C. R. Jacob, T. A. Wesolowski, and E. J. Baerends, J. Phys. Chem. A 109, 7805 (2005).

[21] C. R. Jacob, J. Neugebauer, and L. Visscher, J. Comput. Chem. 29, 1011 (2008).

[22] G. te Velde, F. M. Bickelhaupt, E. J. Baerends, S. J. A. van Gisbergen, C. Fonseca Guerra, J. G. Snijders, and T. Ziegler, J. Comput. Chem. 22, 931 (2001).

[23] C. Fonseca Guerra, J. G. Snijders, G. te Velde, and E. J. Baerends, Theoret. Chem. Acc. 99, 391 (1998).

[24] ADF2013, SCM, Theoretical Chemistry, Vrije Universiteit, Amsterdam, The Netherlands, http://www.scm.com (access date: 06/06/2014).

[25] Y. Zhao and D. G. Truhlar, J. Phys. Chem. A 110, 13126 (2006).

[26] J. P. Perdew, Phys. Lett. A 165, 79 (1992).

[27] E. V. Lenthe and E. J. Baerends, J. Comput. Chem. 24, 1142 (2003).

[28] L. A. Thomas, Proc. Camb. Phil. Soc. 23, 542 (1927).

[29] E. Fermi, Z. Phys. 48, 73 (1928).

[30] P. Cortona, Phys. Rev. B 44, 8454 (1991).

[31] A. Lembarki and H. Chermette, Phys. Rev. A 50, 5328 (1994).

[32] O. Christiansen, H. Koch, and P. Jørgensen, Chem. Phys. Lett. 243, 409 (1995).

[33] C. Hättig and F. Weigend, J. Chem. Phys. 113, 5154 (2000).

[34] C. Hättig, Adv. Quantum Chem. 50, 37 (2005). 
[35] G. D. Purvis and R. J. Bartlett, J. Chem. Phys. 76, 1910 (1982).

[36] O. Christiansen, H. Koch, and P. Jørgensen, J. Chem. Phys. 105, 1451 (1996).

[37] O. Christiansen, H. Koch, P. Jørgensen, and J. Olsen, Chem. Phys. Lett. 256, 185 (1996).

[38] K. Aidas, C. Angeli, K. L. Bak, V. Bakken, R. Bast, L. Boman, O. Christiansen, R. Cimiraglia, S. Coriani, P. Dahle, E. K. Dalskov, U. Ekström, T. Enevoldsen, J. J. Eriksen, P. Ettenhuber, B. Fernández, L. Ferrighi, H. Fliegl, L. Frediani, K. Hald, A. Halkier, C. Hättig, H. Heiberg, T. Helgaker, A. C. Hennum, H. Hettema, E. Hjertenæs, S. Høst, I.-M. Høyvik, M. F. Iozzi, B. Jansik, H. J. A. Jensen, D. Jonsson, P. Jørgensen, J. Kauczor, S. Kirpekar, T. Kjærgaard, W. Klopper, S. Knecht, R. Kobayashi, H. Koch, J. Kongsted, A. Krapp, K. Kristensen, A. Ligabue, O. B. Lutnæs, J. I. Melo, K. V. Mikkelsen, R. H. Myhre, C. Neiss, C. B. Nielsen, P. Norman, J. Olsen, J. M. H. Olsen, A. Osted, M. J. Packer, F. Pawlowski, T. B. Pedersen, P. F. Provasi, S. Reine, Z. Rinkevicius, T. A. Ruden, K. Ruud, V. V. Rybkin, P. Sałek, C. C. M. Samson, A. S. de Merás, T. Saue, S. P. A. Sauer, B. Schimmelpfennig, K. Sneskov, A. H. Steindal, K. O. Sylvester-Hvid, P. R. Taylor, A. M. Teale, E. I. Tellgren, D. P. Tew, A. J. Thorvaldsen, L. Thøgersen, O. Vahtras, M. A. Watson, D. J. D. Wilson, M. Ziolkowski, and H. Ågren, Wiley Interdisciplinary Reviews: Computational Molecular Science 4, 269 (2014).

[39] R. Ahlrichs, M. Bär, M. Häser, H. Horn, and C. Kölmel, Chem. Phys. Lett. 162, 165 (1989).

[40] F. Aquilante, L. De Vico, N. Ferré, G. Ghigo, P.-Å. Malmqvist, P. Neogrády, T. B. Pedersen, M. Pitoňák, M. Reiher, B. O. Roos, L. Serrano-Andrés, M. Urban, V. Veryazov, and R. Lindh, J. Comput. Chem. 31, 224 (2010).

[41] P. Huang and E. A. Carter, J. Chem. Phys. 125, 084102 (2006).

[42] S. Sharifzadeh, P. Huang, and E. A. Carter, Chem. Phys. Lett. 470, 347 (2009).

[43] G. Ghigo, B. O. Roos, and P.-Å. Malmqvist, Chem. Phys. Lett. 396, 142 (2004).

[44] N. Forsberg and P.- Å. Malmqvist, Chem. Phys. Lett. 274, 196 (1997).

[45] F. Aquilante, P.-Å. Malmqvist, T. B. Pedersen, A. Ghosh, and B. O. Roos, J. Chem. Theory Comput. 4, 694 (2008).

[46] A. Bergner, M. Dolg, W. Küchle, H. Stoll, and H. Preuß, Mol. Phys. 80, 1431 (1993).

[47] We employ the original Stuttgart/Cologne $(4 s 4 p) /[2 s 2 p]$ basis sets for $C, N$, and $\mathrm{O}$, removing the additional diffuse $p$ functions from the $(4 \mathrm{~s} 5 \mathrm{p}) /[2 \mathrm{~s} 3 \mathrm{p}]$ basis of $\mathrm{O}$. We then add the $d$ polarization and the $s, p$, and $d$ diffuse functions from the aug-cc-pVDZ basis set for all atoms, obtaining a contraction of $(5 \mathrm{~s} 5 \mathrm{p} 2 \mathrm{~d}) /[3 \mathrm{~s} 3 \mathrm{p} 2 \mathrm{~d}]$. 
[48] CHAMP is a quantum Monte Carlo program package written by Umrigar, C. J.; Filippi, C. and collaborators.

[49] M. Burkatzki, C. Filippi, and M. Dolg, J. Chem. Phys. 126, 234105 (2007).

[50] For the hydrogen atom, we use a more accurate BFD pseudopotential and basis set. Dolg, M.; Filippi, C., private communication.

[51] We add one $s$ and one $p$ diffuse function on the carbon and the nitrogen using exponents from the aug-cc-pVDZ basis set, taken from EMSL Basis Set Library (http://bse.pnl.gov).

[52] C. Filippi and C. J. Umrigar, J. Chem. Phys. 105, 213 (1996), as Jastrow correlation factor, we use the exponential of the sum of three fifth-order polynomials of the electron-nuclear (e-n), the electron-electron (e-e), and of pure 3-body mixed e-e and e-n distances, respectively. The Jastrow factor is adapted to deal with pseudo-atoms, and the scaling factor $\kappa$ is set to 0.60 a.u.

[53] M. Schmidt, K. K. Baldridge, J. A. Boatz, S. T. Elbert, M. S. Gordon, H. J. Jensen, S. Koseki, N. Matsunaga, K. A. Nguyen, S. Su, T. L. Windus, M. Dupuis, and J. J. A. Montgomery, J. Comput. Chem. 14, 1347 (1993).

[54] C. J. Umrigar, J. Toulouse, C. Filippi, S. Sorella, and R. G. Hennig, Phys. Rev. Lett. 98, 110201 (2007).

[55] C. Filippi, M. Zaccheddu, and F. Buda, J. Chem. Theory Comput. 5, 2074 (2009).

[56] M. Casula, Phys. Rev. B 74, 161102 (2006).

[57] M. Casula, S. Moroni, S. Sorella, and C. Filippi, J. Chem. Phys. 132, (2010).

[58] W. L. Jorgensen, J. Chandrasekhar, and J. D. Madura, J. Chem. Phys. 79, 926 (1983).

[59] M. Humbert-Droz, X. Zhou, S. V. Shedge, and T. A. Wesolowski, Theor. Chem. Acc. 133, 1405 (2014).

[60] T. Yanai, D. P. Tew, and N. C. Handy, 393, 51 (2004).

[61] S. Fux, C. R. Jacob, J. Neugebauer, L. Visscher, and M. Reiher, J. Chem. Phys. 132, $164101(2010)$.

[62] P. W. Ayers and M. Levy, Phys. Rev. A 80, 012508 (2009).

[63] P. W. Ayers, M. Levy, and A. Nagy, Phys. Rev. A 85, 042518 (2012). 


\section{Chapter 5}

\section{Multiscale excited-state modeling of green fluorescent protein: Solving a polarizing issue ${ }^{\dagger}$}

We study the absorption properties of green fluorescent protein (GFP) using several multiscale schemes. For the modeling of the excitation on the photoactive chromophore, we employ a variety of electronic-structure methods: Time-dependent density functional theory (TDDFT), multiconfigurational perturbation theory (NEVPT2 and CASPT2), and quantum Monte Carlo (QMC), and we treat the rest of the protein at several different levels to explore the most important physical effects: Nonpolarizable and polarizable classical descriptions and quantum environments (DFT embedding). After performing thermal sampling using ab initio dynamics for both protonation forms, we choose a total of 100 room-temperature frames and treat these at the lowest class of environmental description, static point charges. From these results, we identify an important difference between the protonation states: The excitation of the neutral A form is mostly tuned by the coordinates of the chromophore, while the anionic B form is more affected by the external parameters of the hydrogen-bond network. Given that these initial runs yield blue shifted results compared to the experiments, we pursue various ways of going beyond the pointcharge models. Through comparisons with TDDFT reference calculations on a 300atom cluster, we find that the point charges cause an average blue shift of about 0.1 $\mathrm{eV}$ and correcting this error is not possible through adding ground-state polarizable dipoles. On the other hand, we show that dipoles induced in the excited state alleviate the errors yielded by the point-charge description, but only in the formulation of linear-response polarization (polLR) and not that of state-specific ones (polSS). Since apparently non-electrostatic coupling (captured by polLR but not polSS) with the environment is the most important effect, the dominantly electrostatic DFT embedding does not improve on the static point-charge model.

$\dagger$ This chapter is submitted for publication as C. Daday, C. Curutchet, A. Sinicropi, B. Mennucci, and C. Filippi, "Chromophore-protein coupling beyond non-polarizable models: Understanding absorption in green fluorescent protein ", 


\subsection{Introduction}

The development of multiscale methods to treat complex environments is at the forefront of research in computational chemistry [1-6]. An important application of these approaches is the description of the excited states of large photosensitive systems, where the excitation of a limited active region is modeled at a high-level electronic structure method and a lower level of theory describes the rest of the system. While several possible combinations of computational ingredients are possible in such hierarchical models, the simplest embedding approach of a static classical environments in so-called quantum mechanics in molecular mechanics (QM/MM) calculations has seen widespread use for more than a decade $[1,3]$. There is however mounting evidence that this approach is inadequate for the treatment of excitation energies: Several recent papers using classical point charges in combination with a variety of quantum approaches [7-13] have reported significant errors in the computed excitation energies with respect to experimental absorption maxima in different photoactive proteins.

There are essentially two main paths one may follow to alleviate the shortcomings stemming from the inadequacy of classical point charges. One may either employ a more sophisticated but still classical environment or attempt to treat parts of the environment at the quantum level. In the first class of models, the use of polarizable dipole moments (MMpol) [14-17] is gaining popularity: Polarizable dipoles appear to yield significantly improved excitation energies with respect to non-polarizable classical embedding [13,18-21] and offer the appealing feature that the effect of including dipoles induced in the ground state or also responding to the excitation of the embedded system can be systematically studied [22]. The second class of models, seeking to treat more atoms at a quantum level, has several possible members. In the so-called "mechanical embedding" schemes, the high-level quantum calculation is only performed on an isolated part of the total system and determines a correction to a low-level supermolecular quantum calculation [23]. A self-consistent quantum method is sub-system density functional theory (DFT) embedding, where the environment density creates a one-electron embedding potential to be used in a high-level calculation of the active sub-system. Similarly to the case of polarizable dipoles, ground-state $[24,25]$ and state-specific [26] schemes are available to study the effect of the environment response. Finally, a more "brute-force" approach $[10,13,27]$ is to simply increase the quantum region to include part of the protein environment and treat the whole cluster at the same level of theory. The clusters might however be much larger than what is viable with most quantum methods: A recent study [8] reported that more than 700 quantum atoms are necessary to converge the excitation energy of the photoactive yellow protein.

Here, we focus on wild-type Aequorea green fluorescent protein (GFP), as a particularly ideal case, which challenges our understanding of how the the excitation of the photoreceptor (the chromophore) couples to the protein environment, and of what the necessary ingredients in a computational method are to describe its photophysics. The importance of GFP as photobiological system stems from being the progenitor of the large family of intrinsically fluorescent proteins $[28,29]$ which have 
launched a revolution in cell biology being compatible with non-invasive imaging in living cells. More recently, the development of fluorescent proteins with targeted photochemical properties has also enabled remarkable advances in super-resolution bio-imaging techniques capable of beating the diffraction limit [30,31]. Relevantly to the purpose of the current study, the environment surrounding the chromophore in these proteins is known to play a critical role in tuning its excited-state behavior: Through the mutagenis and continuous discovery of GFP-like proteins in different sea organisms [28], this class of proteins now covers almost the entire visible spectrum both in emission and absorption. On the other hand, predicting the relation between relatively fine structural changes in the chromophore pocket and the spectral properties of the chromophore-protein complex remains a very demanding task for multiscale approaches. Several recent studies [12, 19,32] have attempted a computational characterization of these photoactive systems but reproduced trends in absorption between the various proteins with limited degree of success.

For wild-type GFP, in particular, many computational articles have appeared in the literature $[7,9,12,13,18-21,27,32-40]$ due to the importance of the system and abundance of experiments characterizing its structural and spectral properties. The bulk of these studies produced however excitation energies blue-shifted with respect to absorption experiments when using a classical static treatment of the protein $[7,9,12,32,34-40]$. Consequently, the focus has recently shifted towards improving the description of the environment either through polarizable dipoles [18-21] or simply by increasing the quantum region and therefore treating all atoms at the same level of theory $[10,27]$. While both approaches appear to improve on experimental agreement, many fundamental questions remain unanswered that transcend the choice of GFP as system of study: Does the excitation of the chromophore polarizes the environment and how long range is this effect? Is the coupling between the active site and the protein of electrostatic nature or do we ultimately need a fully quantum mechanical description? Are there clear signatures indicating which computational route one must follow in modeling other photobiological systems?

Here, we seek to provide robust answers to these questions and, to this aim, employ a large battery of tools to describe the chromophore-protein complex. We will begin with extensive QM/MM molecular dynamics simulations and generate a large number of representative frames (about 100 in total) to investigate the interplay between structure and excited states of both protonation states of GFP. Analyzing numerous frames allows us to faithfully assess temperature effects, explore the main factors that determine the spread of absorption energies, and avoid the danger of drawing far-reaching conclusions on a single configuration of the system. We will consider the three main approaches to multiscale modeling of excited states, namely, static point charges, polarizable dipoles, and DFT embedding (the latter being here applied for the first time to fluorescent proteins) and, for many configurations sampled from the dynamics, either compare their results to supermolecular calculations on very large clusters or change the quantum method within the same hybrid scheme to understand how the response varies with the sophistication of the quantum treatment. For this purpose, we will perform calculations with several different electronic-structure methods: Time-dependent density functional theory (TDDFT) 
with two different functionals and three wave-function methods, that is, the complete active space perturbation theory (CASPT2), n-electron valence state perturbation theory (NEVPT2), and quantum Monte Carlo (QMC). In particular, we will explore the nature of polarization effects on the excitation energies of GFP using a frozen and a polarizable environment both at the level of DFT and MMpol embedding to identify the origin of the failure of a static classical description. Finally, we will establish the validity of representing the whole protein as a small cluster in several different ways; this analysis has been lacking in the bulk of previous literature.

The remainder of this paper is organized as follows: In Section 5.2, we describe the computational details and, in Section 5.3, we present our main results. We discuss them and draw our conclusions in Section 5.4.

\subsection{Computational details}

For the QM/MM simulations [41, 42], we use the CPMD 3.17.1 [43] and Gromos96 [44] codes with the Amber 03 [45] force field and the TIP3P [46] water model for the $\mathrm{MM}$ atoms. We treat the $\mathrm{QM}$ region with density functional theory (DFT) and employ the PBE [47,48] exchange-correlation functional, the MartinsToullier pseudopotentials [49] and a plane-wave cutoff of $70 \mathrm{Ry}$. The size of the quantum box is such that the distance between periodic replicas is at least $8 \AA$. We perform the Car-Parrinello molecular dynamics (MD) simulations with a time step of 4 a.u (about $0.1 \mathrm{fs}$ ) and a fictitious electron mass of 400 a.u. For the first 0.5 ps of the room-temperature $(300 \mathrm{~K})$ runs, we use the Berendsen thermostat [50] with a time constant of 10 a.u. We then switch to a Nosé-Hoover thermostat $[51,52]$ with a characteristic frequency of $2000 \mathrm{~cm}^{-1}$ and default settings for the rest of the 27 and 25 ps MD simulations for B and A form, respectively. In the annealing runs, we rescale the velocities at each step by 0.999 until the temperature is below 0.1 and 10 $\mathrm{K}$ for the $\mathrm{A}$ and the $\mathrm{B}$ form, respectively, and by 0.99 afterwards.

We use Molcas 7.4 and 7.8 [53] for the CASPT2 calculations and a module adapted from the Molcas-Embed interface developed by Carter and co-workers [54, 55] for the CASPT2/DFT embedding runs. We employ the ANO-S-VDZP [56, 57] basis set and, in the convergence tests, the aug-cc-pVDZ [58]. We use the Cholesky decomposition of the two-electron integrals [59] with a threshold of $10^{-4}$. In the CASPT2 calculations, we always adopt the default IPEA zero-order Hamiltonian [60] with an additional constant imaginary shift [61] of 0.1 a.u. and, unless otherwise noted, report the excitation energies computed at the single-state level. We also freeze as many $\sigma$ orbitals as there are heavy atoms. In the MM calculations, we describe the protein with the Amber 99 [62] force field and set the charges of the charge group closest to the MM boundary to zero, redistributing them to the next charge group, weighted by nuclear charge. All CASSCF calculations are carried out in state average and we use a CAS $(14,13)$ for the B form and CAS $(16,15)$ for the A form. The reason why we deviate from the minimal CAS $(16,14)$ is different for the two forms: For the A form, we found that including an extra virtual orbital to substantially stabilize our states and for the B form, freezing one occupied orbital 
allowed for cheaper calculation on the large number of frames that we will consider.

We also perform CASPT2/MMpol calculations in the presence of a polarizable environment using the Amber pol12 parameters (AL model in Ref. $[63,64]$ ) to describe the protein and the water solvent. For water, we derived the point charges from a standard RESP fit using the electrostatic potential computed at the MP2/augcc-pVTZ level on the TIP3P water geometry $\left(\mathrm{q}_{\mathrm{O}}=-0.726, \mathrm{q}_{\mathrm{H}}=0.363\right)$. These CASPT2/MMpol results are estimated using a two-step strategy recently presented [65], where the MM induced dipoles and charges from a CASSCF/MMpol calculation performed using the Gaussian code are later used in Molcas as a static external potential to obtain the CASPT2 results. Such CASSCF/MMpol calculations, performed using a locally modified version of Gaussian09, revision A.02 [66], are obtained using the state-average procedure for the ground and first excited states and adapting the MM polarization either to the ground state (polGS), or to the ground and excited states in two separate calculations (polSS).

The time-dependent density functional theory (TDDFT) runs are also performed using a locally modified version of Gaussian09, revision A.02 [66], and with the CAM-B3LYP [67] and LC-BLYP [68] functionals. We use the AL Amber pol12 parameters $[63,64]$ for the polarizable dipole calculations and Amber 99 for the nonpolarizable runs for consistency with CASPT2/MM. We employ the 6-31G [69] basis set on the hydrogens and the $6-31+\mathrm{G}(\mathrm{d})[69-71]$ for the other atoms.

To generate the embedding potentials for the CASPT2/DFT and TDDFT/DFT calculations, we use the ADF 2013.2 code [72-74] with the DZP [75] basis set. We employ the M06HF [76] exchange-correlation functional for the intramolecular calculations, and the PW91k [77] kinetic functional and the PW91 [78] exchangecorrelation potential for the non-additive part of the embedding potential. For frozendensity embedding TDDFT/DFT calculations, we use the ADF program with the same embedding potentials used in the CASPT2 runs and the CAMY-B3LYP functional $[67,79,80]$.

For the NEVPT2 calculations in the strongly contracted formulation [81-83], we use ORCA 3.0.2 [84] with the ANO-L-VDZP [56, 57] basis set and the aug-ccpVTZ [58] as auxiliary basis set for the resolution of identity [85]. The RIJCOSX approximation [86] is employed in the CASSCF step. The orbital energies for the doubly occupied and virtual orbitals appearing in the Dyall Hamiltonian [87] (used for the definition of the zero order Hamiltonian in NEVPT2) were obtained by the diagonalization of a generalization of the Fock operator [88] (canonical orbital option in ORCA). In the construction of the third- and fourth-order density matrices, the CASSCF wave function is truncated so that configurations with lower weight than a threshold of $10^{-8}$ are discarded. The NEVPT2/MM runs are performed in the presence of the same point charges as in the CASPT2 calculations.

For the QMC calculations, we use CHAMP [89] with scalar-relativistic energyconsistent Hartree-Fock pseudopotentials and the corresponding cc-pVDZ [90,91] basis sets augmented with diffuse $s$ and $p$ functions on the heavy atoms [92]. We employ a two-body Jastrow factor to account for electron-electron and electronnucleus correlations and use different Jastrow factors to describe different types of atoms [93]. The starting orbitals are obtained in a state-average CASSCF calcula- 
tions with Molcas 7.8 (in the presence of the MM charges, if appropriate) and are not optimized in the QMC runs. We truncate the CAS expansion in state-average natural orbitals with a cutoff on the CI coefficients as detailed in Section 5.3. The CI coefficients and Jastrow parameters are optimized with the linear method [94] in a state-averaged fashion [95] within variational Monte Carlo (VMC). The pseudopotentials are treated beyond the locality approximation [96] and an imaginary time step of 0.05 or 0.075 a.u. is used in the diffusion Monte Carlo (DMC) calculations. The potential generated by the external MM charges is put on a grid which is padded to have at least $5.0 \AA$ distance from any atom in the chromophore and has a step size of 0.2 a.u. A finer grid with a step size of 0.1 a.u. yields VMC excitation energies compatible to better than $0.02 \mathrm{eV}$. The electron loss ratio (attempted evaluations outside the cubic grid) is less than $10^{-6}$ in all runs. All QMC calculations reported below refer to DMC.

\subsubsection{Models}

To prepare the structures of the A and B forms, we start from the protein models obtained in our previous study [9]. For the A form, we remove one GFP barrel from the previously used dimeric system as well as all water molecules which are more than $5 \AA$ from the remaining monomer. We then solvate the system and bring the added water in equilibrium by performing a 5 ns NPT classical MD simulation with NAMD [97] while keeping all other atoms fixed. The solvation box is approximately $72 \times 84 \times 82 \AA^{3}$ for the A form and $70 \times 65 \times 70 \AA^{3}$ for the B form.

In the QM/MM simulations, we set the boundary between the $\mathrm{QM}$ and $\mathrm{MM}$ regions at the single bonds $\mathrm{C}_{\mathrm{OOH}}-\mathrm{C}_{\alpha}$ of Phe64 and $\mathrm{N}-\mathrm{C}_{\alpha}$ of Val68. With this QM part of 40 (A form) or 39 (B form) atoms, we run long QM/MM room-tempertature simulations of 27 and 25 ps for the A and B form, respectively. We then perform a cluster analysis [98] with VMD [99] on 4000 frames sampled every 5 fs from the last $20 \mathrm{ps}$ of the QM/MM trajectories. Each cluster is populated by frames that are within a certain cutoff distance of the central frame of the same cluster. As distance between two frames, we employ the mass-weighted fitted RMSD where the frames are first aligned to obtain the lowest possible RMSD. In the computation of the distance between two frames, we include all the atoms in the protein but not the water molecules, and use a cutoff distance of $0.5 \AA$ for the A form and $0.6 \AA$ for the $\mathrm{B}$ form to define a cluster. The central frames of the most populated 10 clusters from the cluster analysis are then used in the calculation of the excitation energies. For further analysis, we isolate 50 equidistant frames sampled every 400 fs from the QM/MM MD runs and also determine a final annealed structure for both forms of the protein.

For the B form, we also consider a larger QM region, which includes the chromophore and the residues Gln94 and Arg96, and the boundary between the QM and $\mathrm{MM}$ regions also includes the single bonds $\mathrm{C}_{\mathrm{OOH}^{-}}-\mathrm{C}_{\alpha}$ of Val93, N-C $\mathrm{C}_{\alpha}$ of Thr97, and $\mathrm{C}_{\alpha}-\mathrm{C}_{\beta}$ of Glu95 (the backbone of Glu95 is modeled at the quantum level but its sidechain classically). With the resulting QM region, containing 93 atoms, 5 of which are capping hydrogens, we perform a QM/MM simulation of $1.5 \mathrm{ps}$ and determine then an annealed structure. 


\begin{tabular}{|c|c|c|c|}
\hline Cluster & atoms & Residues & Capping \\
\hline Cluster 1 & 168 & $\begin{array}{l}\text { CRO, Thr62, Gln69, Gln94, Arg96, His148, } \\
\text { Val150, Ile167, Thr203, Ser205, Glu222, } \\
8 \mathrm{xH}_{2} \mathrm{O}\end{array}$ & $\mathrm{C}_{\beta}$ or deeper \\
\hline Cluster 2 & 279 & $\begin{array}{l}\text { CRO, Thr62, Gln94, Arg96, Tyr145, His148, } \\
\text { Thr203, Ser205, Glu222, 4x } \mathrm{H}_{2} \mathrm{O}\end{array}$ & next $\mathrm{C}_{\alpha}$ \\
\hline Cluster 3 & 345 & As Cluster 1 & next $C_{\alpha}$ \\
\hline Cluster 4 & 529 & $\begin{array}{l}\text { CRO, Leu42, Val61, Thr62, Thr63, Gln69, } \\
\text { Cys70, Phe71, Gln94, Arg96, Asn121, } \\
\text { Tyr145, His148, Val150, Phe165, Ile167, } \\
\text { Gln183, Thr203, Ser205, Glu222, 8xH }{ }_{2} \mathrm{O}\end{array}$ & $\operatorname{next} \mathrm{C}_{\alpha}$ \\
\hline
\end{tabular}

Table 5.1: Number of atoms and residues involved in the quantum clusters considered for GFP. The type of capping is also given.

Finally, we construct QM cluster models for the TDDFT, CASPT2/DFT, and TDDFT/DFT calculations. The smallest cluster contains 168 atoms from 10 residues (with deep cuts at $\mathrm{C}_{\beta}$ or even deeper) and 8 water molecules, and is similar to the models used in Ref. 12. For the three larger clusters of 279, 345, and 529 atoms (4 waters and 8 residues, 7 waters and 10 residues, and 7 waters and 19 residues, respectively), we use capping methyl groups and cut the residues at the neighboring $\mathrm{C}_{\alpha}$ atoms in the backbone. The list of residues included in the clusters is given in Table 5.2.1.

\subsection{Results}

In equilibrium, the chromophore of wild-type GFP exists in either a neutral A form or an anionic B form. Upon excitation in the blue, the A form of the chromophore loses the phenolic proton converting to an intermediate anionic I form, which in turn infrequently transforms into the thermodynamically stable B form [100]. The neutral chromophore is known to transfer its proton to Glu222 through the wire CRO-WatSer205-Glu222 [101, 102] but other differences in the surroundings are difficult to assess experimentally: Since the predominant form of wild-type GFP at room temperature is the neutral one, the structure of the anionic form cannot be established through X-ray diffraction. As workaround, one can adopt as starting model a suitably modified X-ray structure of the mutant S65T which stabilizes the anionic form as also done in earlier computational work [9,103]. The available X-ray structures of this mutant (PDB entries 1EMA, 1EMG, and 1Q4A [104-106]) suggest that the hydrogen-bond network of the B form is similar to that of the A form except for a different orientation of Thr203. A recent theoretical study [40] challenges the accepted orientation of Thr203 and also suggests a new conformation of the anionic chromophore. We will explore this possibility and its consequences in Subsection 5.3.5.

The solvated chromophore of GFP does not exhibit fluorescence at room temperature (quantum yield of less than 0.001 [107]) but fluoresces in solution at 77 
$\mathrm{K}[108,109]$ and also in the protein, being rigidly held by the environment which inhibits its numerous available modes of relaxation [109-111]. Temperature effects on the chromophore and its surroundings have nevertheless an impact on the excited-state properties of GFP: When temperature is reduced, the absorption maximum moves from 2.59 to $2.63 \mathrm{eV}$ for the $\mathrm{B}$ form, and from 3.12 to $3.05 \mathrm{eV}$ for the A form and, consequently, the distance between the peaks of two forms decreases from 0.5 to $0.4 \mathrm{eV}$. Furthermore, the width of the peaks is much smaller in the lowtemperature than in the room-temperature spectrum [112]. We begin our study by investigating the effects of temperature on the chromophore-protein complex of both forms of GFP and explore the range of conformations visited by the system in our $\mathrm{QM} / \mathrm{MM}$ MD trajectories.

\subsubsection{Thermal stability of hydrogen-bond network}

For the A form, the hydrogen-bond network around the chromophore is quite stable during the 27 ps of QM/MM dynamics. In particular, the CRO-WAT-Ser205-Glh222$\mathrm{CRO}$ wire is always connected except for a very short instance in the equilibration part where the water loses the H-bond with Ser205 (see Figure 5.1 for the position of the residues with respect to the chromophore and the labeling). His 148 is always bonded to the water close to the phenolic oxygen and also the imidazolinone ring has a stable environment. In particular, O12 is bonded to Arg96, Gln94, and a water molecule, while N2 is weakly bonded to a water molecule. Only the O12-water interaction is sporadically broken. The annealed frame has the same hydrogen-bond network as the one in the dynamics.

For the B form, we observe instead that the hydrogen-bond network adopts several configurations even during the relatively short 25 ps dynamics as can be seen in Figure 5.2. The CRO-WAT-Ser205-Glu222-CRO wire is occasionally broken as the water molecule loses the bond to the phenolic O1. There are in fact four residues around $\mathrm{O} 1$ that compete to form hydrogen bonds with it: Tyr145, Hid148, Thr203, and a water molecule as illustrated in Figure 5.1. Through the trajectory, either two or three of these four residues are bonded to the phenolic oxygen. In particular, in most of the trajectory, exclusively one of the two residues Tyr145 and His148 (see Figure 5.2) is bonded to the oxygen, while Thr203 is bonded most of the time only rarely losing the bond due to the movement of the $\mathrm{H}$ atom (the heavy atoms of the residue are always at a distance consistent with a hydrogen bond). Finally, we note that the water molecule close to the phenolic oxygen $\mathrm{O} 1$ forms a bond with Thr203 for a short time interval, losing the one with $\mathrm{O} 1$ but keeping the other hydrogen bonded with Ser205. The annealed frame of the B form has Tyr145 bonded to the phenolic $\mathrm{O} 1$ even though, for most of the dynamics, it had His148 instead. This simply indicates that multiple close minima are available to the system. In fact, the annealing run with the larger quantum region results instead in Tyr145, His148, and the water bonded to the phenolic ring, while Thr203 forms a bond with the carbonyl group in His148, mainly due to the movement of the gamma hydrogen atom in the threonine residue. Consequently, the commonly used approach of using a single frame obtained through annealing or optimization can lead to a configuration which 


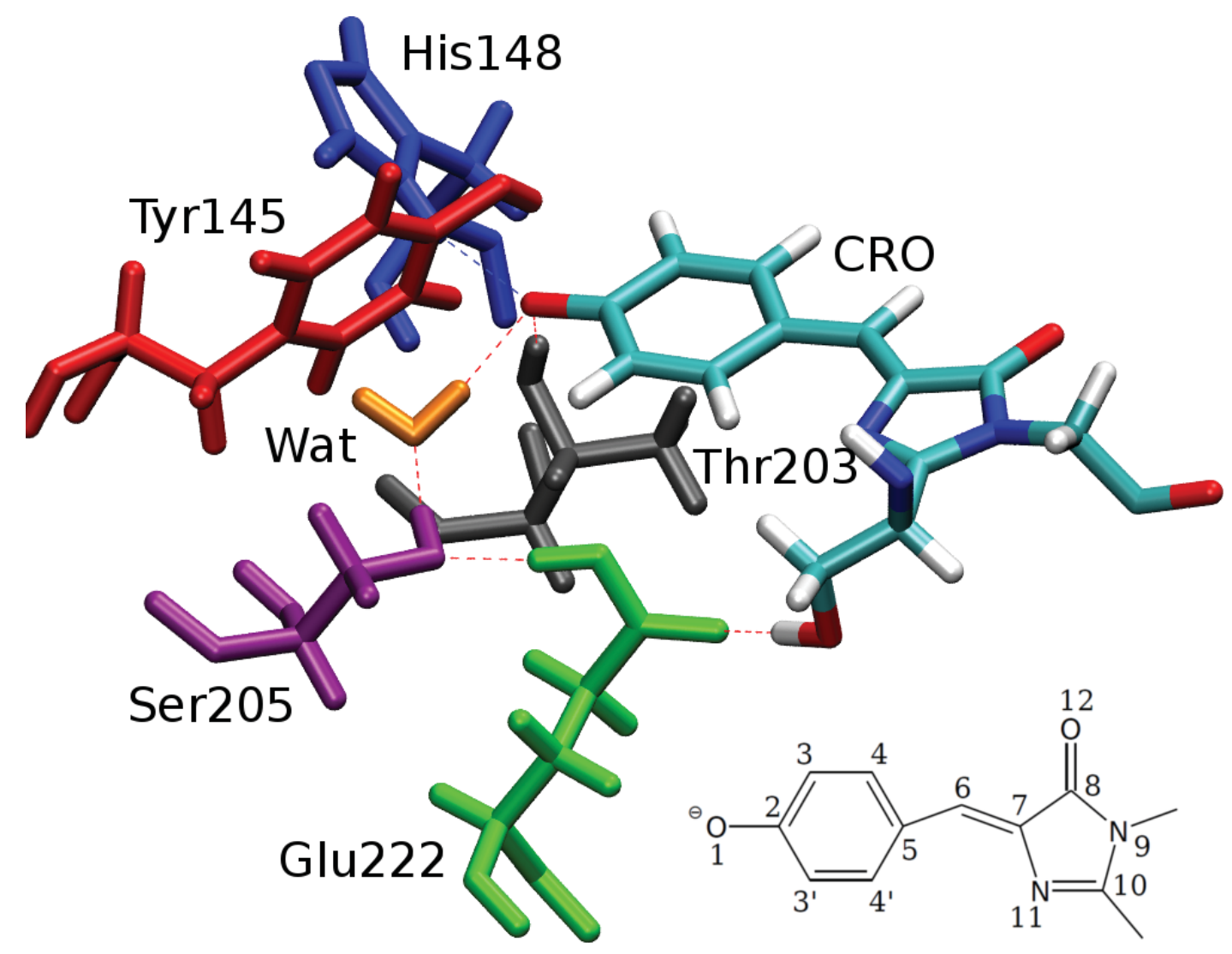

Figure 5.1: B form: The hydrogen-bond network around the chromophore (CRO). The phenolic oxygen $\mathrm{O} 1$ is bonded to the water molecule (orange), His148 (red), Thr203 (gray), while Tyr148 (red) does not participate in this particular snapshot. The hydrogen-bond network is completed by Ser205 (purple) and Glu222 (green). The hydrogen-bond network of the A form is similar but Glu222 loses its proton to $\mathrm{O} 1$ and Thr203 is no longer bonded to $\mathrm{CRO}$ owing to a change in conformation. 

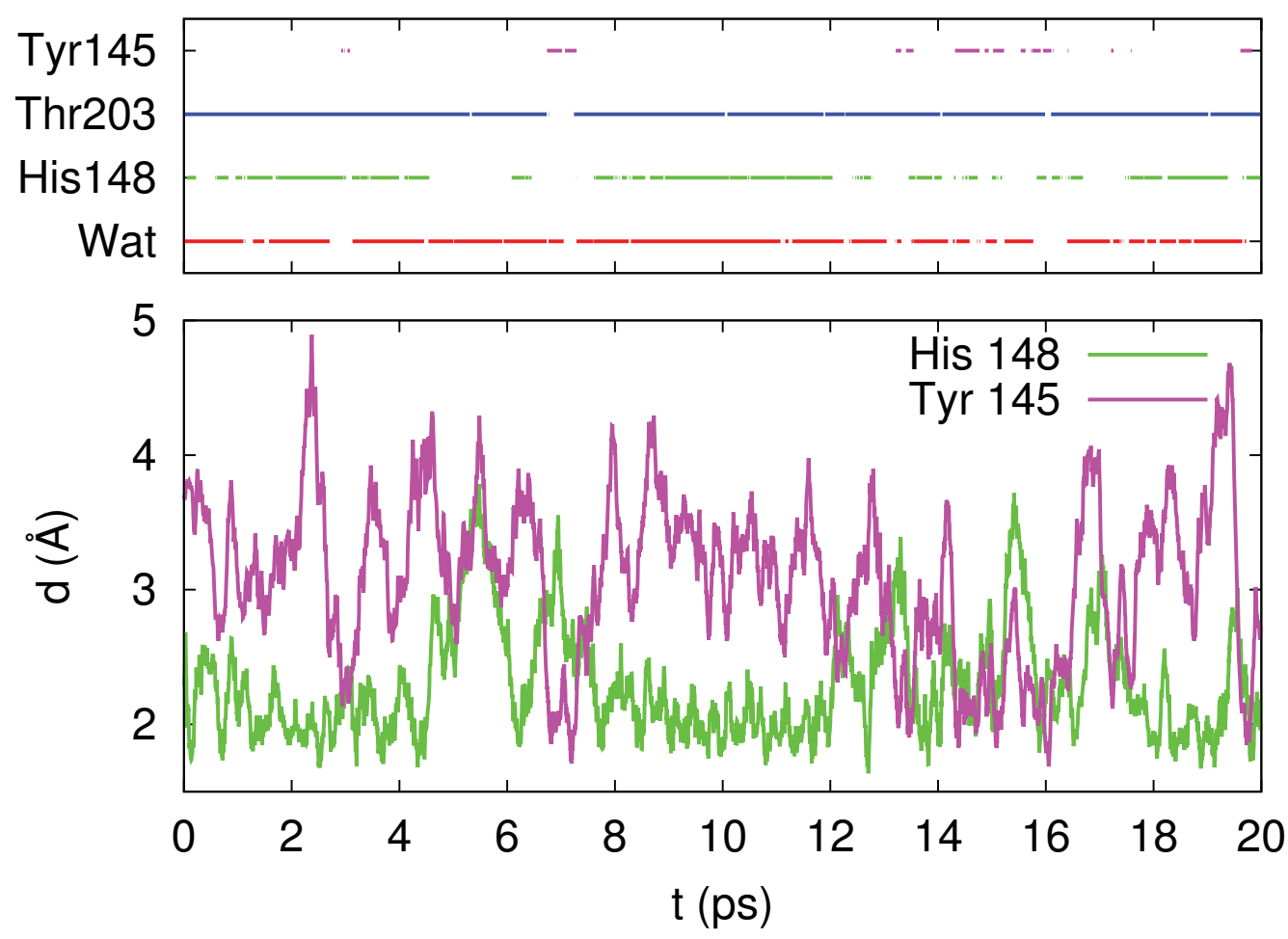

Figure 5.2: B form: The hydrogen bonds formed with the phenolic oxygen of the chromophore during the QM/MM trajectory as a function of time. Top: The bonding of the four possible residues (when the distance between their hydrogen atom and $\mathrm{O} 1$ is less than $2.3 \AA$ ). Bottom: The distances between $\mathrm{O} 1$ and the bonded hydrogen atoms of Tyr145 and His148 as a function of time.

is not representative of the average behavior.

\subsubsection{Relationship between structure and excitation ener- gies}

To analyze the excitation energies over a wide range of representative conformations of the protein, we choose 50 equidistant frames from the last $20 \mathrm{ps}$ of the $\mathrm{QM} / \mathrm{MM}$ trajectories of both the A and B forms (i.e. one frame every $500 \mathrm{fs}$ ). Given the significant number of frames, we perform a first screening of the behavior of the excitation energies over these frames using the relatively cheap TDDFT/MM method. We choose CAM-B3LYP as exchange-correlation functional since it has been shown $[113,114]$ to ameliorate problems with spurious charge-transfer effects that may plague conventional hybrid methods $[115,116]$. Later in this paper, we will also employ more sophisticated methods to treat either the chromophore or the protein environment and focus then on 10 representative frames obtained from cluster analysis for each protonation state. Our findings on the 50 frames for the A and B forms are summarized in Figure 5.3. The A form exhibits a spread in the TDDFT excitation energies of more than $0.5 \mathrm{eV}$ and a standard deviation of $0.1 \mathrm{eV}$, while the 


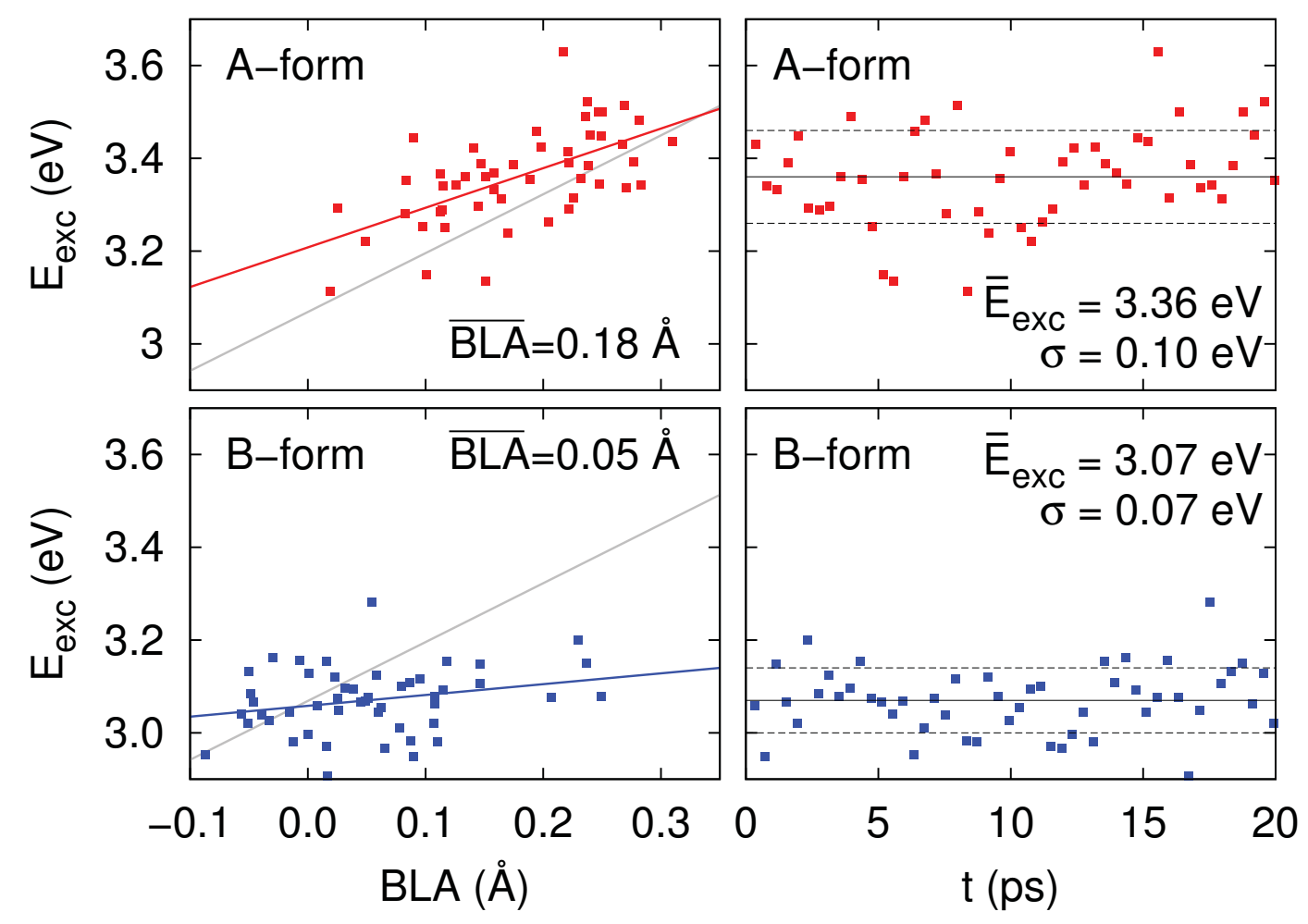

Figure 5.3: The dependence of the TDDFT/MM excitation energies of the A (top) and B (bottom) forms on time (right) and on the bond-length alternation (left). In the BLA panels, the colored linear fit refers to the specific protonation form, while the gray line is a fit of all data for both protonation forms.

B form has a smaller but still considerable spread of almost $0.4 \mathrm{eV}$ and a slightly smaller standard deviation of $0.07 \mathrm{eV}$. The extent of values for the A form is particularly surprising since the protein environment is very stable during the dynamics as noted above.

The origin of these large variations is worth exploring. Previous studies [12,34, 117] have shown that the excitation energies of fluorescent proteins depend on the bond-length alternation (BLA) within the chromophore. The BLA was then defined as a linear combination of the 15 heavy-atom bonds in the chromophore, whose weights were fitted to the excitation energies in various ways. We also perform such an analysis but use a much simpler definition of the BLA than in previous works, namely,

$$
\begin{aligned}
\mathrm{BLA} & =\left[\mathrm{O} 1 \mathrm{C} 2+\frac{1}{2} *\left(\mathrm{C} 3 \mathrm{C} 4+\mathrm{C} 3^{\prime} \mathrm{C} 4^{\prime}\right)\right. \\
& +\mathrm{C} 5 \mathrm{C} 6+\mathrm{C} 7 \mathrm{C} 8] \\
& -\left[\mathrm{O} 12 \mathrm{C} 8+\frac{1}{2} *\left(\mathrm{C} 2 \mathrm{C} 3+\mathrm{C} 2 \mathrm{C} 3^{\prime}\right)\right. \\
& \left.+\frac{1}{2} *\left(\mathrm{C} 4 \mathrm{C} 5+\mathrm{C} 4^{\prime} \mathrm{C} 5\right)+\mathrm{C} 6 \mathrm{C} 7\right]
\end{aligned}
$$

where the atom labeling is given in Figure 5.2. The significance of this parameter can be understood in terms of the two resonance structures of the anionic chromophore 

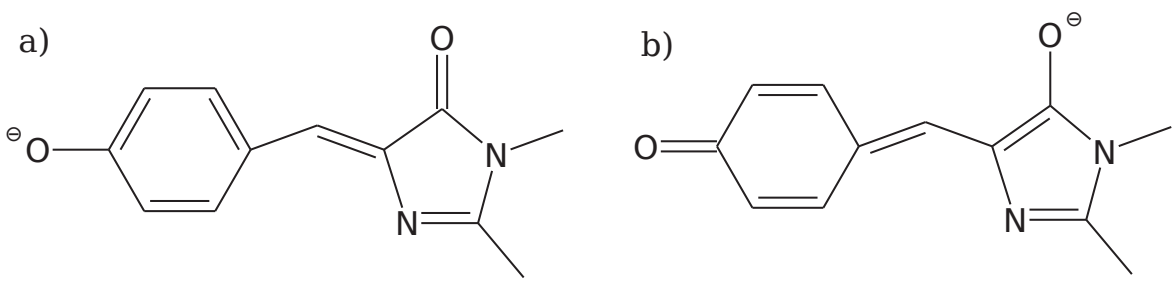

Figure 5.4: The a) benzenoid and b) quinoid resonance structures of the anionic chromophore.

shown in Figure 5.4: The BLA defined above is positive when the chromophore is in the dominant benzenoid structure and negative in the quinoid one. Such a simple definition avoids the danger of over-fitting our data and, as shown in Figure 5.3, suffices to describe a sizable part of the tuning in the A form. In this case, since the chromophore adopts exclusively the benzenoid resonance structure, when the BLA approaches zero through thermal fluctuations, the electronic structure of the ground state is severely perturbed, thereby closing the gap. On the other hand, for the anionic B form, small values of the BLA are more typical since the quinoid structure acquires importance and the ground state is more capable of adjusting also to conformations with a negative BLA and a dominant quinoid resonance structure. The dependence on the BLA of the excitation energy is therefore much less steep as shown in Figure 5.3.

We also note that the quality of the fit for the $\mathrm{B}$ form is lower, indicating that other factors than the BLA affect its excitation energy. We can in fact improve the fit if we account for environmental effects by adding for instance fitting parameters which measure the presence or lack of a bond between $\mathrm{O} 1$ and His148 and/or Tyr145 (the resulting shifts are +0.05 and $+0.03 \mathrm{eV}$, respectively). Even though more data would be required to elucidate the precise role of the different amino acids in the surrounding of the chromophore, our analysis suggests that the spread of the absorption energies has different causes in the two forms: For the A form, it is mostly determined by the internal coordinates (which in turn are coupled to the external conformations) and, for the B form, the flexible hydrogen-bond network around the chromophore seems to be a more directly relevant factor.

While the average BLA is smaller for the B form than the A form, this fact alone is not enough to explain why the A form has a higher excitation energy. If we attempt to fit the dependence of the excitation energies on the BLA for both forms together as done for instance in Ref. [34], we observe that the excitation energies of the A form are systematically higher than those of the B form as is evident from Figure 5.3 and that a single fit is inadequate (we reach the same conclusion if we allow arbitrary weights for the bonds in the fitting procedure). We therefore add an energy shift between the two forms in the linear model to account for this offset, which is found to be $0.22 \mathrm{eV}$ and improves the quality of the fit as shown in Figure 5.5. Consequently, of the total shift of $0.29 \mathrm{eV}$ between the average excitation energies of the A and $\mathrm{B}$ forms, only $0.07 \mathrm{eV}$ is accounted for by a lower average BLA in the B form than in the $\mathrm{A}$ form and the remaining $0.22 \mathrm{eV}$ is caused by the different electronic structure 


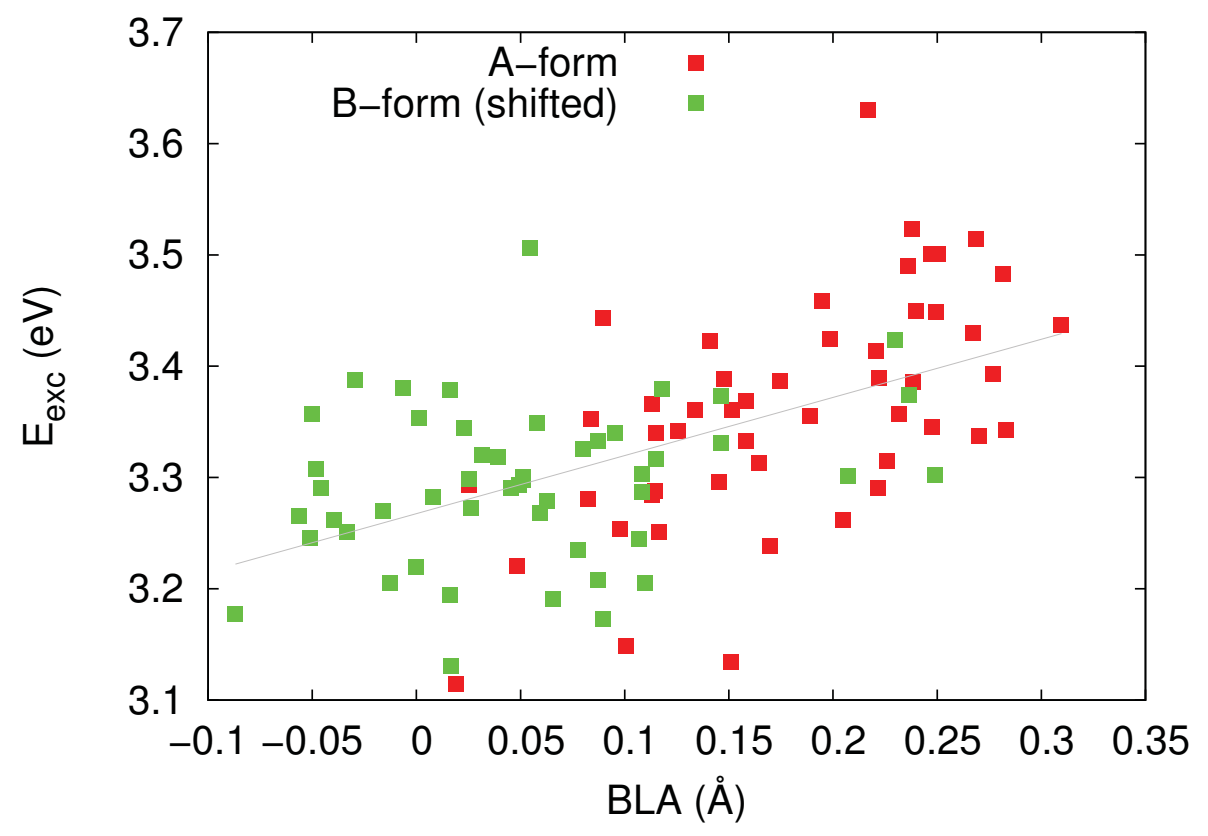

Figure 5.5: $\mathrm{A}$ and $\mathrm{B}$ forms: TDDFT/MM excitation energies as a function of the BLA. A shift between the two forms is introduced as parameter to yield the best linear fit, the value (A-B) being $0.22 \mathrm{eV}$. (This value is not directly comparable with the experimental value of $0.4 \ldots 0.5 \mathrm{eV}$ since the average BLA is different in the two protonation forms.)

of the two protonation states. We note in passing that, for both forms, the excitation energy displays the strongest dependence on the bridging distance C6-C7 (double bond in the A form and predominantly double bond in the B form) when we search for correlations between the excitation and any single bond length, while the other bridging distance, C5-C6, has a less significant influence. This is in line with the Hückel model description [37] for the excitation energy of the A form, which is approximated as a simple ethylene-like HOMO-LUMO transition on the C6-C7 bond. However, the same model assumes the bonds C5-C6 and C6-C7 to be equivalent in the B form, which does not fit our findings.

In agreement with previous studies [12, 19,39, 118], we find that CAM-B3LYP gives blue-shifted values for the excitation energies of these photoactive systems. For the B form, the average excitation energy over 50 frames is $3.07 \pm 0.01$, which is almost $0.5 \mathrm{eV}$ blue-shifted with respect to the experimental absorption maximum of $2.59 \mathrm{eV}$. Furthermore, the dependence of the excitation energies on the BLA is not as clear as for the A form and attempts to correlate the spread in the energies with environmental changes gave inconclusive results. Given these relatively unclear findings, one can ask whether the observed trends are physically meaningful or just noise due to the use of approximate TDDFT. To answer this question, we carry out CASPT2/MM calculations on all 50 frames of the B form and compare the resulting excitation energies with those obtained with CAM-B3LYP/MM. As illustrated in 


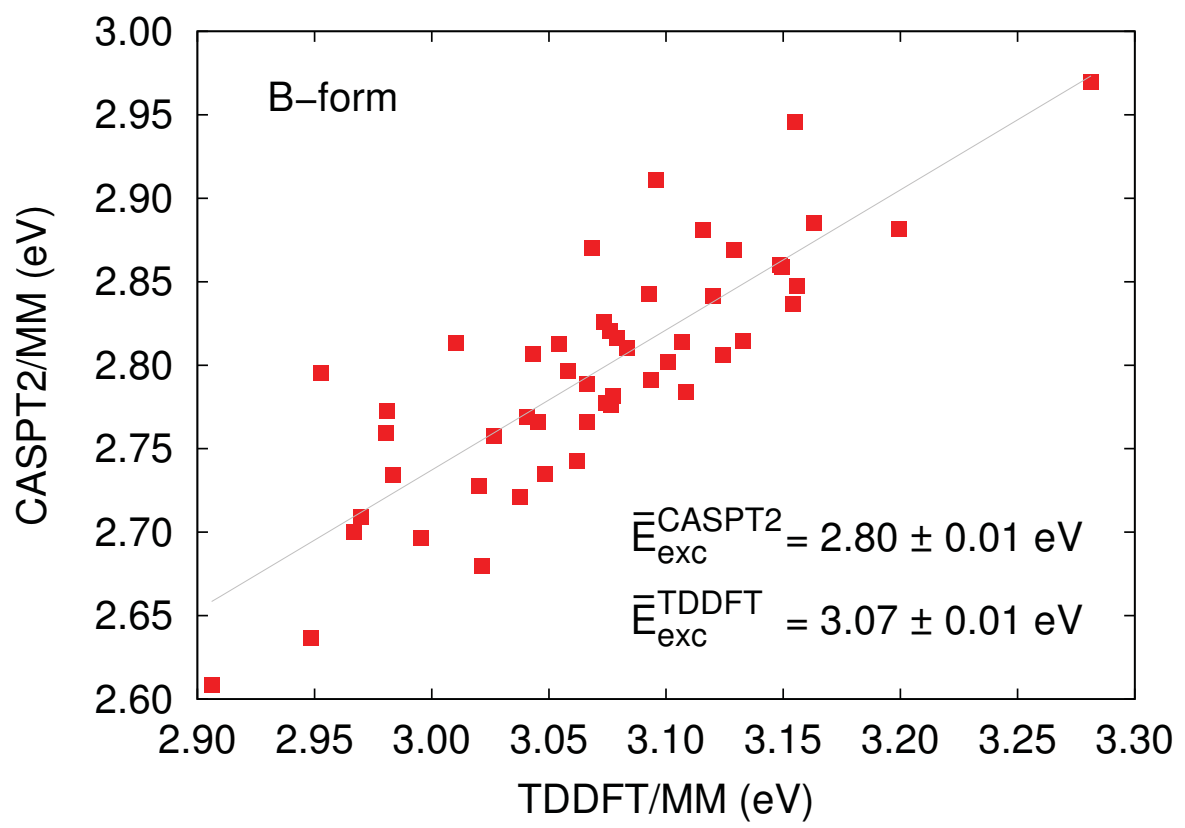

Figure 5.6: B form: Comparison of the TDDFT/MM and CASPT2/MM excitation energies computed on the 50 equidistant frames of the QM/MM MD simulation.

Figure 5.6, the conclusion is unequivocal: The two methods are in excellent agreement as regards general trends. While these calculations reinforce our preceding analysis (the BLA dependence is qualitatively identical for CASPT2 energies), the CASPT2/MM average excitation energy of $2.80 \pm 0.01 \mathrm{eV}$ is still clearly blue-shifted compared to experiment. This means that thermal sampling by itself is not sufficient to overturn the strong indications coming from previous studies $[9,11,39,119]$ that describing the protein environment as only classical charges produces an unphysical blue shift in the excitation energies of related photoactive systems.

To verify that the choice of quantum method is not responsible for the blue shift, we extend our investigation to other computational approaches for 10 representative frames obtained through cluster analysis. We employ the LC-BLYP functional in the TDDFT calculations and also use quantum Monte Carlo and NEVPT2 as alternative wave function-based methods. To pinpoint the effect of the MM environment, we consider the difference between the QM/MM description and the excitation energies computed on the isolated chromophore at the geometry obtained within the protein. As shown in Figure 5.7, the trends across the frames are qualitatively similar at all levels of theory but the shifts induced by the protein are larger for the wave function approaches. As regards the absolute excitation energies with the other WF methods, we find that NEVPT2 and QMC are rather close and about $0.2 \mathrm{eV}$ blue-shifted compared to CASPT2, thereby further worsening the agreement with experiments. This finding renders even more pressing the search for an alternative, better description of the environment, which will be the focus of the following subsection. 


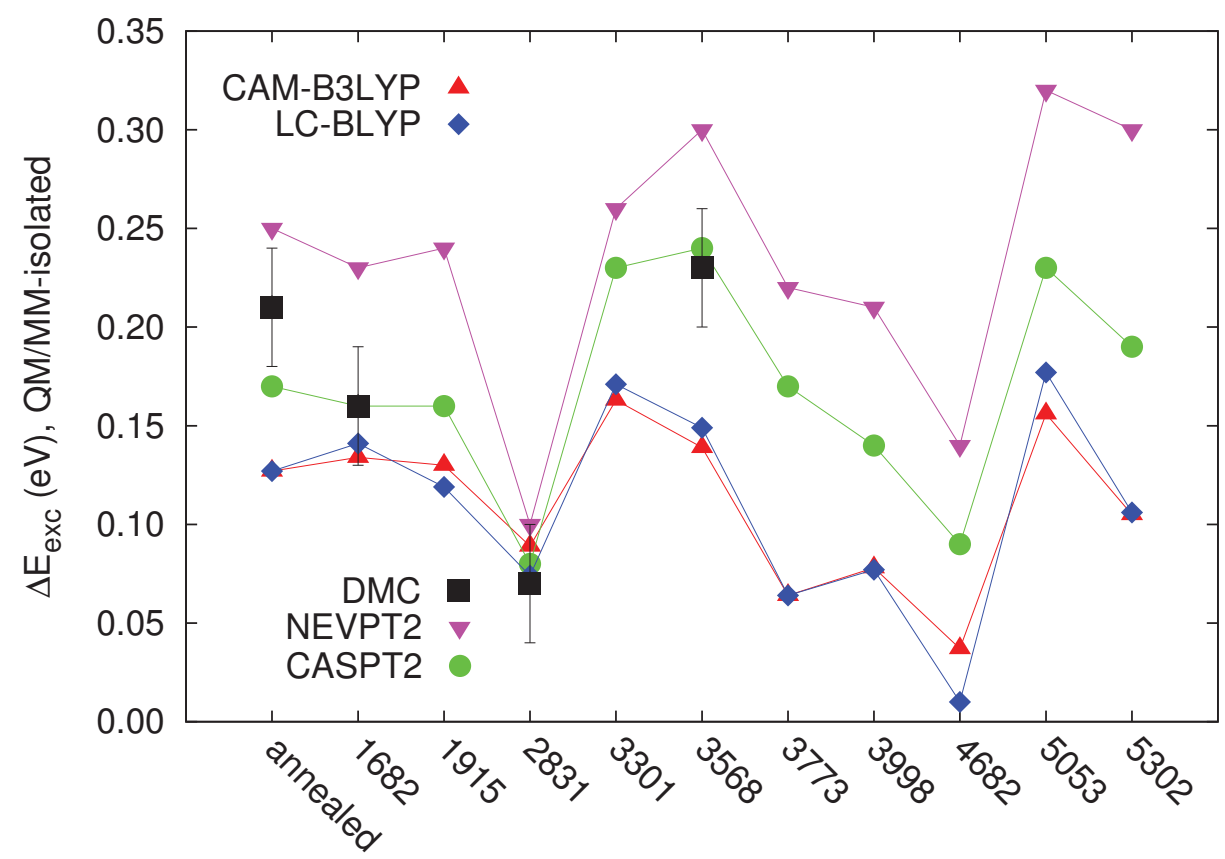

Figure 5.7: B form: Difference between the QM/MM and isolated excitation energies computed with TDDFT (CAM-B3LYP and LC-BLYP), CASPT2, NEVPT2, and QMC.

\subsubsection{Improved description of the environment}

To further investigate whether the MM description of the protein is responsible for the significantly blue-shifted excitation energies with respect to experiment, we explore here the use of other embedding methods to improve on the simple MM scheme with static point charges. A possible strategy is that of adding a polarizable embedding (MMPol) in terms of induced dipoles [15]. From the QM/MM trajectories of the $\mathrm{A}$ and the $\mathrm{B}$ forms, we choose 10 representative snapshots using cluster analysis and repeat the TDDFT investigation for these frames with induced dipoles in addition to the MM charges to describe the protein. On these 10 frames we have applied three different MMPol approaches: In the first one, the polarization of the environment is limited to the ground state (polGS), in the second a state-specific (polSS) polarization is used in terms of the response of the induce dipoles to the change in the density of the chromophore due to the excitation, and in the third linear response (polLR) is instead used where the induced dipoles are determined by the chromophore transition density. We note that polSS formulation corresponds to what is called corrected LR method within polarizable continuum approaches [120]. The three models represent three different approximations of the environment response to excitations, namely, polGS neglects any response, i.e. it assumes a completely frozen environment, while polSS and polLR allow the polarizable part of the environment to respond to the excitation. In particular, polSS introduces a relaxation of the environmental polarization to the new excited-state density, while polLR uses a resonance-like formalism where the transition density of the chromophore oscil- 
lating at the Bohr frequency induces an oscillating polarization in the environment. This creates in turn an in-phase response on the chromophore which gives on average a nonzero interaction with the transition density itself. The LR formulation has been largely used in combination with polarizable continuum models and has been described as a dispersion-like term [121].

As summarized in Figure 5.8, we find that for the A form, polarizing the environment around the ground state (polGS) of the chromophore leaves the excitation energy essentially unchanged with respect to the non-polarizable MM approach. On the other hand, for the B form, polGS leads to a blue shift $(0.06 \mathrm{eV})$. That the ground-state polarization has a larger effect on the $\mathrm{B}$ form is understandable as the chromophore is charged. Moving to the two descriptions which introduce an environment response to the excitation, we obtain that, for both forms, the polSS description does not significantly change the excitation energy with respect to a completely frozen solvent (polGS). This seems to indicate that the variation of the electron density upon excitation is not very significant neither in the A nor the and B form (as a matter of fact, the TDDFT/CAM-B3LYP absolute variation of the dipole moment between ground and excited state is around 3 Debye for both molecules. On the contrary, the polLR causes a substantial red shift with respect to polGS for both the A $(-0.17 \mathrm{eV})$ and the $\mathrm{B}(-0.22 \mathrm{eV})$ forms. Such a finding can be explained in terms of the large transition dipole which characterizes the excitation in both forms and which can strongly interact with the polarizable environment. Perturbatively to linear order, the shifts in the polSS and polLR excitation energies with respect to polGS can in fact be shown [122] to be proportional to the square of the change in dipole moment between the ground and excited states and to the square of the transition dipole moment, respectively, a relation which is very convincingly demonstrated in Figure 5.9. From this analysis, we conclude that the chromophore-environment interactions in the ground state are almost equally described by the non-polarizable and the polarizable models. To account for full response to the excitation, we need instead to move beyond an electrostatic-only picture of the coupling with the protein.

An alternative method for treating the environment is DFT embedding [123], which one would expect to be superior to a classical treatment. To generate the embedding potential for the chromophore, we use here either the density of the isolated environment, relaxing the chromophore density [124], or bring the environmental and chromophore densities at self-consistency after an appropriate number of socalled freeze\&thaw cycles [125]. For the A form, we find that DFT embedding with freeze\&thaw cycles yields a small blue shift (about $0.02 \mathrm{eV}$ ) compared to the MM description, while using the density of the isolated environment leaves the excitation energies essentially unchanged. However, for the B form, DFT embedding with freeze\&thaw cycles causes a blue shift with respect to the MM charges as large as $0.15 \mathrm{eV}$ at the level of TDDFT/DFT. Since DFT embedding is conceptually a ground-state embedding method, this finding is reminiscent of the blue shift caused by the polGS model, although the shift is larger for DFT embedding. If one uses the density of the isolated environment, there is almost no shift with respect to the MM values. Therefore, DFT embedding gives at best excitation energies equivalent to the MM ones but only with an isolated environmental density, while one would 

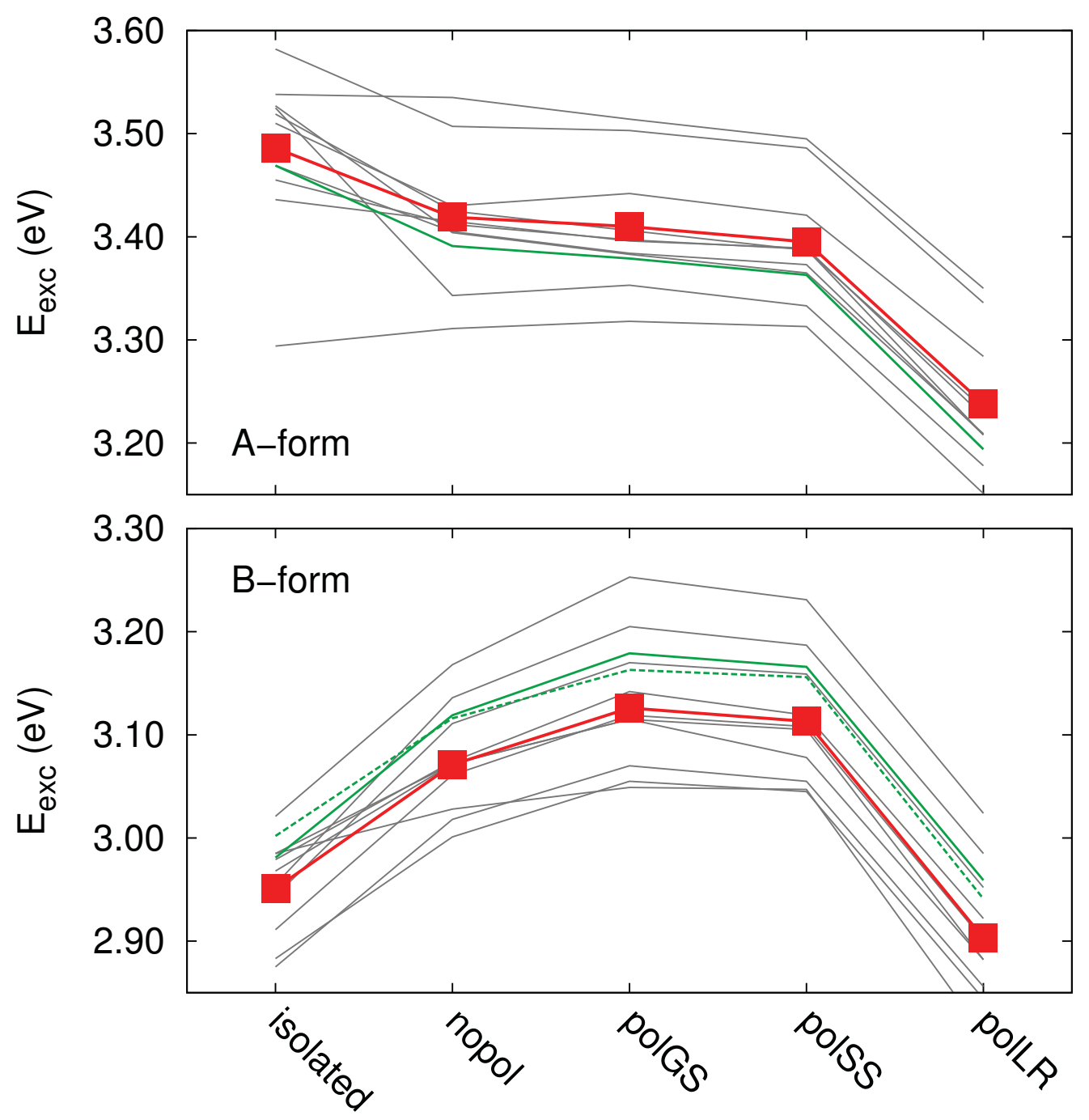

Figure 5.8: A and B forms: Excitation energies computed with TDDFT on the chromophore only (isolated) or surrounded by the environment described with static point charges (nopol), with point charges and dipoles polarized in the ground state (polGS), in the excited state (polSS) or using a linear-response (polLR) formulation. The thin, gray lines represent the results on a specific frame, while the thick, red line shows average values across the frames. The thin, green lines represent the annealed frame, while the dashed, green line for the B form represents the annealed frame obtained with a larger QM region in the QM/MM dynamics. 


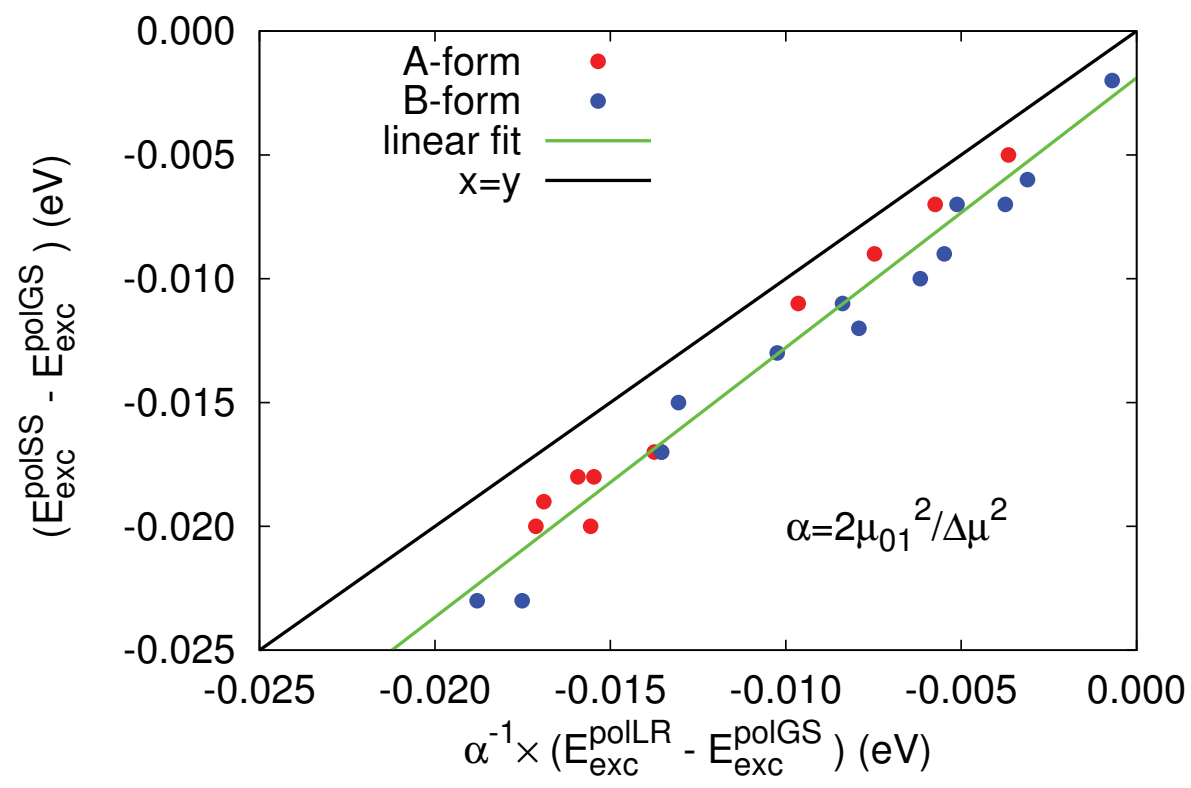

Figure 5.9: A and B forms: Shifts between the TDDFT/MMpol state-specific (polSS) and ground-state polarized (polGS) excitation energies versus the corresponding linear-response (polLR) results rescaled with twice the ratio squared of the transition dipole moment and the change in dipole moment between the ground and excited states.

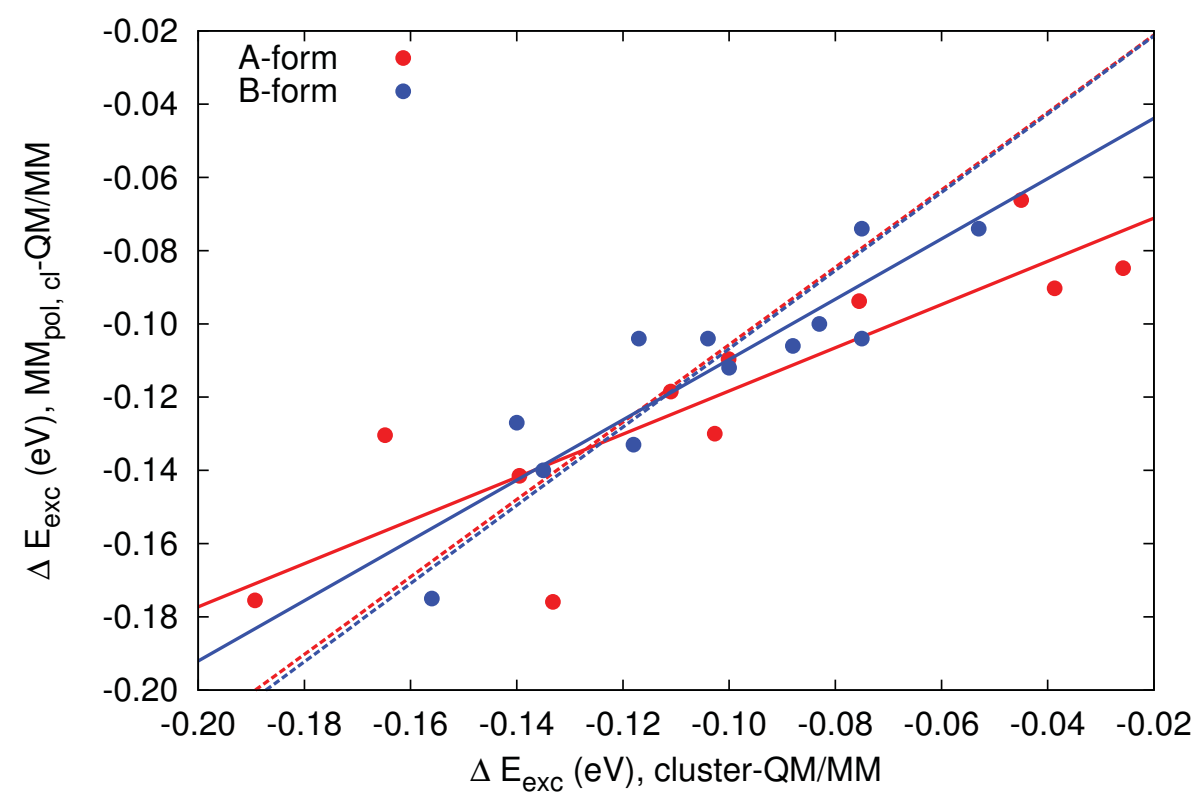

Figure 5.10: A (red) and B (blue) forms: Correlation between the excitation-energy differences cluster-MM and $\mathrm{MMpol}_{\mathrm{Cl}}-\mathrm{MM}(\mathrm{eV})$. Full lines: Fit function $\mathrm{f}(\mathrm{x})=\mathrm{a}^{*} \mathrm{x}+\mathrm{b}$ with coefficients $a=0.59(11), b=-0.059(13)$ for the $\mathrm{A}$ form, and $a=0.82(13), b=-$ 0.027(14) for the B form. Dashed lines: $b=0$ with $a=1.06(9)$ for the A form and 1.07(4) for the B form. 
expect a relaxed environment to provide a better description of the system especially in the anionic B form. Nevertheless, in the following, we present the DFT embedding results computed with the freeze\&thaw scheme which is internally consistent, conceptually related to polGS, and not dictated by the performance of the method for this particular system.

To investigate the effect of polarizing the environment at the DFT level, we also apply the recently introduced state-specific formulation of DFT embedding $[26,126]$ and find that the response to state-specific potentials at the TDDFT/DFT level is on average very small for both forms (see SI). While the use of an excited-state functional in the state-specific scheme would in principle ensure the inclusion of all relevant environmental effects on the excitation, the use of ground-state functionals appears in practice to only capture the electrostatic response of the protein. It is therefore not surprising that the state-specific TDDFT/DFT results are in line with the polSS ones.

While the electrostatic contribution to the environment response is negligible for both forms, polarizable dipoles in linear response appear to offer a possible remedy for the blue shift in the excitation energies caused by the classical point charges. To validate the behavior of polLR in describing the environment effects on the excitations, we compare the polLR results with supermolecular calculations at the same level of theory. Given the size of the protein model and the number of frames, we limit these test calculations to clusters of 279 atoms comprising the chromophore and close-by residues, and compare the TDDFT/MMpol calculations on the same clusters $\left(\mathrm{MMpol}_{\mathrm{Cl}}\right)$ with the supermolecular TDDFT results. As shown in Figure 5.10, we find a remarkably good correlation between the shifts in the polLR and supermolecular excitation energies with respect to the MM description for each frame. In fact, the parameters of a linear fit for the A and B forms are compatible and imposing a intercept of zero in the fit leads to a slope of about one and not a significantly poorer quality of the fit for both forms. Furthermore, the average excitation energies computed at the polLR level on the cluster differ from the average supermolecular reference by less than $0.01 \mathrm{eV}$ for both forms as detailed in Table 5.2.

The recent study of Ref. [13] with the CC2 method reports a somewhat larger discrepancy of about $0.04 \mathrm{eV}$ between linear-response MMpol and supermolecular calculations both done on 160-atom clusters of the A and B forms. This is in line with our results: When considering individual frames in Figure 5.10, one can find some frames with similar TDDFT/MMpol excitation energies but a spread of up to $0.05 \mathrm{eV}$ in the cluster values, and vice versa. Averaging over many frames seems however to lead to a high degree of cancellation in the MMpol errors.

A summary of the results obtained for all 10 frames by applying the different ways of treating the environment in combination with a TDDFT description of the excitations is reported in Figure 5.11 and in Table 5.2, where we also list the excitation energies obtained with the LC-BLYP functional. We find that LC-BLYP responds to the changes in the description of the environment very similarly to CAM-B3LYP and that the agreement between the polLR and cluster excitation energies is equally good. We finally note in Table 5.2 and Figure 5.8 that increasing the QM region in the QM/MM dynamics and annealing has no significant effect on the excitation en- 


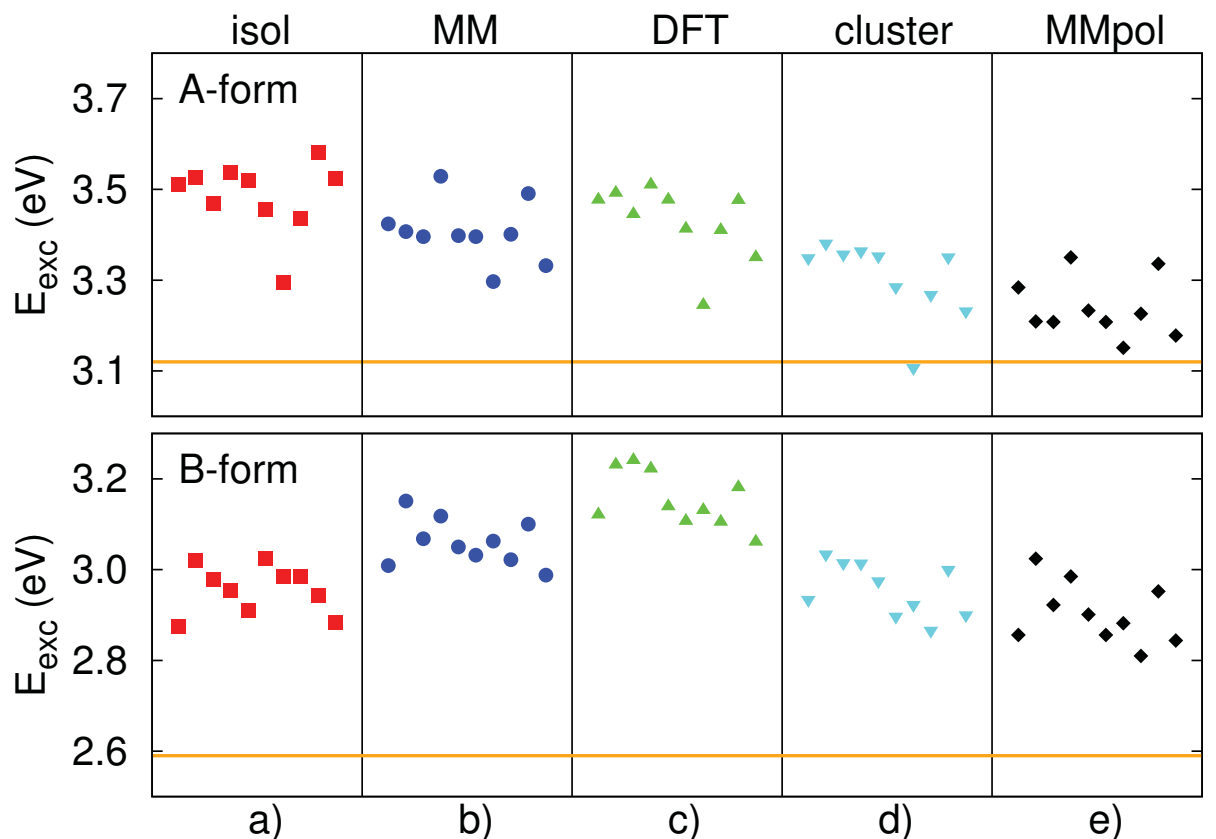

Figure 5.11: A (top) and B (bottom) forms: Excitation energies computed with TDDFT for a) the isolated QM chromophore, b) the QM chromophore/MM protein, c) DFT environment, d) a large QM cluster, e) QM chromophore/MMpol (polLR) protein. The orange lines indicate the room-temperature absorption maxima of 3.12 and $2.59 \mathrm{eV}$ for the $\mathrm{A}$ and $\mathrm{B}$ forms, respectively.

ergies of the B form at variance with the suggestion of Ref. [10], where only a partial and constrained optimization of the structure was carried out.

As already discussed in the previous section, all WF methods considered here agree with TDDFT in predicting that the MM description of the protein leads to significantly blue-shifted excitation energies of the B form with respect to the experimental absorption maximum: The average excitation energy over the 10 frames is $2.79 \pm 0.02$ and $3.02 \pm 0.02 \mathrm{eV}$ for CASPT2/MM and NEVPT2/MM, respectively. The QMC/MM excitation energies on a subset of frames are very close to the NEVPT2 values as illustrated in Figure 5.12 and detailed for a subset of frames in Table 5.3. Also the anomalous blue shift observed with TDDFT/DFT for the B form with respect to the MM embedding is confirmed at the wave function level, with CASPT2/DFT predicting an even larger blue shift of $0.25 \pm 0.02 \mathrm{eV}$ and QMC/DFT a similar correction of $0.11 \pm 0.04 \mathrm{eV}$. The further localization of the excitation energy induced by DFT embedding is possibly due to a too high/steep barrier in the embedding potential induced at the edge of the chromophore by the approximate kinetic functional [126]. Increasing the cluster size worsens the situation, causing further blue shifts at the CASPT2 level as shown in Figure 5.13. As in the case of TDDFT/DFT, we find that the use of state-specific DFT potentials has on average almost no effect on the excitation energies at the CASPT2 level (less than $0.01 \mathrm{eV}$ ).

All findings with WF methods for the B form are summarized in Figure 5.12, 


\begin{tabular}{|c|c|c|c|c|c|}
\hline & \multicolumn{4}{|c|}{ CAM-B3LYP } & \\
\hline & \multicolumn{2}{|c|}{ A form } & \multicolumn{3}{|c|}{ B form } \\
\hline & $300 \mathrm{~K}$ & $0 \mathrm{~K}$ & $300 \mathrm{~K}$ & $0 \mathrm{~K}$ & $0 \mathrm{~K}^{a}$ \\
\hline isolated & $3.49(3)$ & 3.47 & $2.96(2)$ & 2.98 & 3.00 \\
\hline QM/MM & $3.41(2)$ & 3.38 & $3.06(2)$ & 3.11 & 3.11 \\
\hline QM/MMpol & $3.24(2)$ & 3.19 & $2.90(2)$ & 2.96 & 2.94 \\
\hline $\mathrm{QM} / \mathrm{MMpol}_{\mathrm{cl}}$ & $3.30(3)$ & 3.26 & $2.95(2)$ & 3.01 & 2.98 \\
\hline $\mathrm{QM}^{\mathrm{DFT}} \mathrm{cl}$ & $3.43(3)$ & 3.43 & $3.15(2)$ & 3.23 & 3.19 \\
\hline Cluster & $3.30(3)$ & 3.28 & $2.96(2)$ & 3.03 & 2.99 \\
\hline Exp. [112] & 3.12 & 3.05 & 2.59 & 2.63 & 2.63 \\
\hline \multicolumn{6}{|c|}{ LC-BLYP } \\
\hline & \multicolumn{2}{|c|}{ A form } & \multicolumn{3}{|c|}{ B form } \\
\hline & $300 \mathrm{~K}$ & $0 \mathrm{~K}$ & $300 \mathrm{~K}$ & $0 \mathrm{~K}$ & $0 \mathrm{~K}^{a}$ \\
\hline isolated & $3.70(3)$ & 3.67 & $3.03(2)$ & 3.05 & 3.07 \\
\hline QM/MM & $3.58(3)$ & 3.53 & $3.13(2)$ & 3.18 & 3.16 \\
\hline QM/MMpol & $3.41(3)$ & 3.35 & $2.99(3)$ & 3.04 & 3.00 \\
\hline $\mathrm{QM} / \mathrm{MMpol}_{\mathrm{cl}}$ & $3.49(4)$ & 3.42 & $3.03(3)$ & 3.09 & 3.02 \\
\hline $\mathrm{QM}^{\mathrm{DFT}} \mathrm{Cl}_{\mathrm{cl}}$ & - & - & - & - & - \\
\hline Cluster & $3.51(4)$ & 3.48 & $3.06(3)$ & 3.14 & 3.06 \\
\hline Exp. [112] & 3.12 & 3.05 & 2.59 & 2.63 & 2.63 \\
\hline
\end{tabular}

Table 5.2: TDDFT excitation energies (eV) of A and B forms averaged over 10 frames $(300 \mathrm{~K})$ and for the annealed structure $(0 \mathrm{~K})$. The polLR formulation is used in QM/MMpol. Experimental absorption maxima at $295 \mathrm{~K}$ and $1.6 \mathrm{~K}$ are also given. The error on the averages is in brackets.

\begin{tabular}{lcccc}
\hline frame & TDDFT & SS/MS CASPT2 & NEVPT2 & QMC \\
\hline \multicolumn{5}{c}{ A-form } \\
ann. & 3.38 & $3.24 / 3.32$ & - & $3.55(2)$ \\
1189 & 3.42 & $3.36 / 3.43$ & - & $3.69(2)$ \\
2103 & 3.40 & $3.31 / 3.40$ & - & $3.58(1)$ \\
4519 & 3.33 & $3.23 / 3.28$ & - & $3.51(2)$ \\
& \multicolumn{5}{c}{ B-form } \\
ann. & 3.11 & $2.82 / 2.82$ & 3.06 & $3.10(2)$ \\
1682 & 3.01 & $2.72 / 2.73$ & 2.98 & $3.03(2)$ \\
2831 & 3.07 & $2.71 / 2.72$ & 2.92 & $2.95(2)$ \\
3568 & 3.05 & $2.85 / 2.86$ & 3.03 & $3.13(2)$ \\
\hline
\end{tabular}

Table 5.3: A and B forms: Excitation energies (eV) computed with MM embedding at the TDDFT, SS/MS CASPT2, NEVPT2, and QMC levels. The statistical error on the $\mathrm{QMC}$ values is given in brackets. 


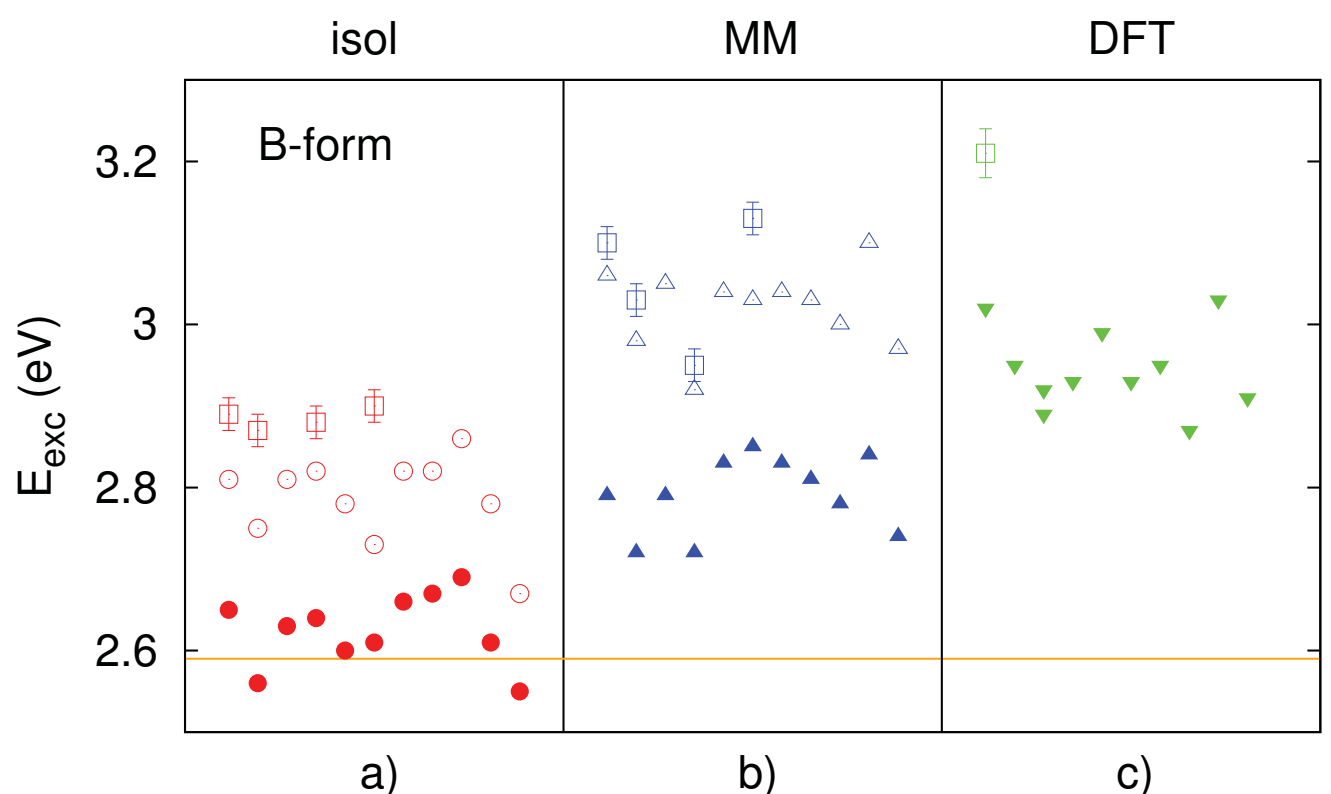

Figure 5.12: B form: Excitation energies computed with WF methods for a) the isolated QM chromophore, b) the QM chromophore/MM protein, c) DFT environment. Full symbols refer to CASPT2, empty squares to QMC, and other empty symbols to NEVPT2. The leftmost symbols in each panel represent the annealed structure and the others the room-temperature frames, while the orange line indicates the experimental room-temperature absorption maximum of $2.59 \mathrm{eV}$.

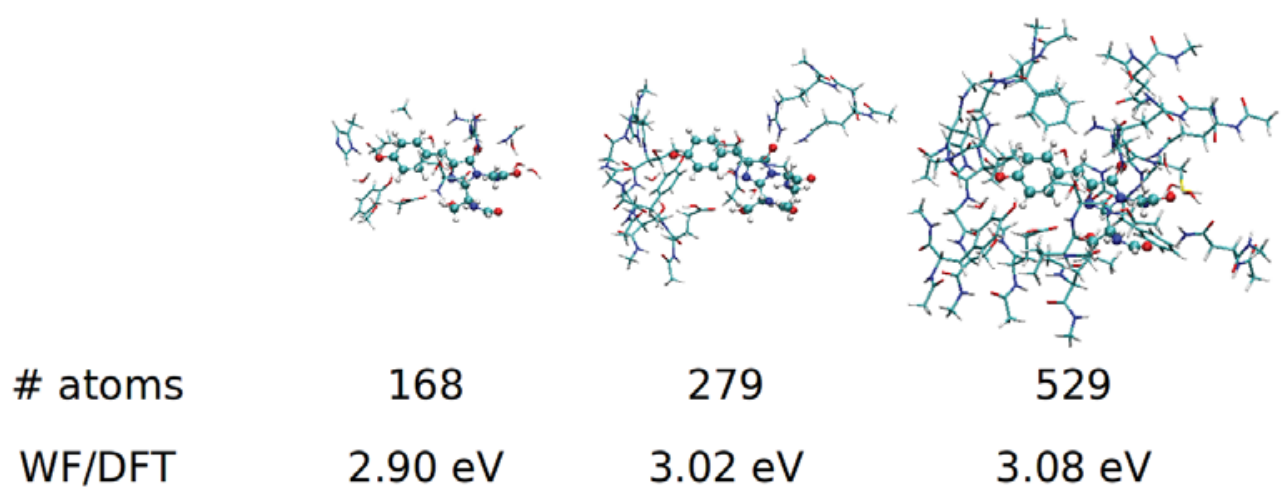

Figure 5.13: B form: Excitation energies computed with CASPT2/DFT for increasing cluster size for the annealed structure. 
which clearly illustrates how the trends for the different embedding methods mirror the TDDFT ones of Figure 5.11, with the difference that the shifts are generally slightly larger for the WF methods. One might therefore hope that the large redshift between TDDFT/MM and TDDFT/cluster or TDDFT/MMpol would be even larger between $\mathrm{WF} / \mathrm{MM}$ and $\mathrm{WF} / \mathrm{MMpol}$ or a possible $\mathrm{WF} /$ cluster run. Unfortunately, WF/MMpol in the polLR formulation is not yet available for the chosen WF methods (an estimate of such effects at the CASPT2/MMpol level will be given in the conclusions), while a calculation on a cluster of almost 300 atoms is out of reach for all but the simplest WF approaches due to prohibitive costs. For the A form, we encounter severe difficulties in the WF calculations due to the presence of multiple minima in the CASSCF reference calculations and a strong sensitivity of the CASPT2 excitation energies on the size of the active space, the minimal but yet large expansion not being sufficient. Therefore, in Table 5.3, we only present CASPT2/MM and QMC/MM results for four frames (the annealed and three room-temperature ones) where the CASPT2 calculations are robust at both the single- and multi-state level as discussed in detail in the SI. The available WF excitation energies are in line with the TDDFT results also for the A form with QMC/MM being a bit higher and CASPT2/MM a bit lower than the corresponding TDDFT/MM excitation energies on the same frames, and both being blue-shifted with respect to the experimental absorption maximum of $3.12 \mathrm{eV}$ also of this form.

If we focus on the embedding MMpol (polLR) approach which all tests indicate as the most reliable for this system, we can only attempt a comparison with experiments at the TDDFT level. Even though a direct comparison of the CAM-B3LYP results to experimental absorption maxima is tenuous as previously noted, we can use the data for each form to validate our account of thermal effects. Since we have performed MMpol calculations on both room-temperature and annealed frames as listed in Table 5.2, comparing shifts between the excitation energies of the two data sets to experimental shifts in the absorption maxima is possible. We find that for the A form, the annealed (frozen) frame has a lower excitation energy than the average of the room-temperature frames by $0.05 \pm 0.02 \mathrm{eV}$, while for the $\mathrm{B}$ form, the annealed frame has a higher excitation energy than the room-temperature average $(0.04 \pm 0.02$ $\mathrm{eV}$ if we focus on the annealing performed with the same QM region). These two shifts are rather close to the experimental values [112] of $0.07 \mathrm{eV}$ red-shift between room- and low-temperature absorption maxima for the A form and $0.04 \mathrm{eV}$ blue-shift for the B form. The precise values of the shifts are difficult to ascertain, as especially the A form has a wide peak, but reassuringly, the direction and order of magnitude is well reproduced by combining an MMpol embedding with our thermal sampling in the QM/MM MD simulations.

\subsubsection{On the use of a cluster representation of the protein}

In the previous subsection, we employed cluster calculations to validate the MMpol approach and to perform the DFT embedding calculations. Quite often in the literature, a cluster representation of the protein is instead used to compute the excitation energies for a direct comparison with experiments and the question therefore arises 
on how large a cluster must be for a converged calculation of the excitation energies. Distressingly, a recent article [8] suggested that clusters as large as 723 atoms are necessary for reliable results on the photoactive yellow protein.

In Table 5.4, we answer this question for wild-type GFP by enhancing the 279atom cluster calculations in several ways, namely, by increasing the cluster size to 345 atoms, including the rest of the protein outside the cluster as point charges, or comparing the TDDFT/MMpol results for the cluster only and for the full protein. We present also an estimate based on CASPT2/DFT calculations on two different cluster sizes for the B form but consider the observed unphysical increase in the blue shift an artifact of the use of approximate kinetic functionals. In the case of TDDFT-based estimates, the error introduced by the use of a 279-atom cluster is small and comparable for both protonation states, between 0 and $0.07 \mathrm{eV}$ for the $\mathrm{A}$ form and between -0.03 and $0.06 \mathrm{eV}$ for the $\mathrm{B}$ form. Therefore, it appears that a cluster of about 300 atoms is sufficient to estimate the excitation energies and, especially, the trends between the two protonation forms of wild-type GFP. On the other hand, smaller clusters as for instance employed in previous coupled-cluster studies of GFP [10] probably suffer from much larger cluster errors and carry therefore a large uncertainty as regards comparison with experiments. For example, adding the rest of the protein in Ref. 10 as standard classical force-field point charges yielded a $0.2 \mathrm{eV}$ blue shift, which is about an order of magnitude larger than the errors we obtain here due to the addition of the $\mathrm{MM}$ protein $(0.01 \mathrm{eV}$ for the $\mathrm{A}$ and $0.03 \mathrm{eV}$ for the B form).

\subsubsection{Alternative H-bond network for the B form}

Our results strongly indicate that a poor description of the environment via classical point charges is mainly responsible for the blue shift observed in the excitation energies with respect to experiments. Nevertheless, we will here also explore the possibility that a drastically different configuration of the chromophore might lead to the desired agreement with experiments. In particular, we will investigate the impact on the excitation energies of the formation of an internal hydrogen bond between $\mathrm{O}_{\gamma}$ in the Ser65 side chain and N11 of the imidazolinone ring. The extra hydrogen bond on the imidazolinone side of the chromophore is expected to stabilize the excited state as the excitation has a partial charge transfer character from the phenolic to the imidazolinone ring, and therefore red shift the excitation energy. (We note that the an analogous internal hydrogen bond network between the Thr65 side chain and the chromophore is observed in the X-ray structure 1Q4B [106] of the S65T mutant.) The distortion of Ser65 however significantly perturbs the surroundings of the chromophore since it is also coupled to a twist of Glu222 from the anti to the syn conformation and, consequently, to a breaking of the proton-transfer wire, so the combined effect on the excitation energy is in fact hard to predict.

Such a structure was recently put forward as the "true" conformation of the B form of wild-type GFP while the standard hydrogen-bond network we have adopted above was assigned to the I form of GFP [40]. This assignment was motivated by the the small blue shift of $0.02-0.04$ (depending on the quantum method) computed be- 


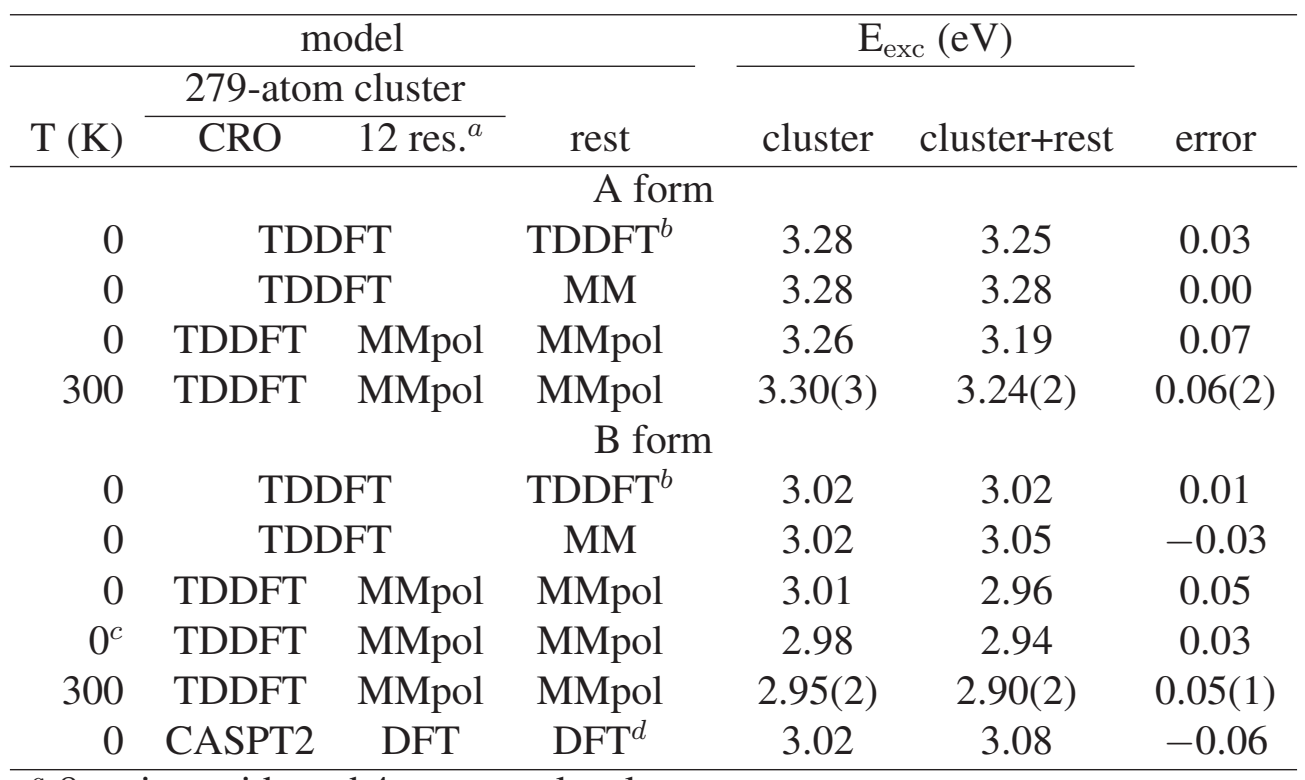

${ }^{a} 8$ amino acids and 4 water molecules.

${ }^{b}$ Enlarging cluster to 345 atoms ( +2 amino acids and 3 waters).

${ }^{c}$ Large QM region in the QM/MM annealing.

${ }^{d}$ Enlarging cluster to 529 atoms (+11 amino acids and 3 waters).

Table 5.4: Cluster convergence analysis for the A and B forms. Results on the chromophore (CRO) plus 12 residues (279-atom cluster) are compared with calculations on a larger cluster (345 and 529 atoms) or the whole protein treated at different levels of theory. 
tween the excitation energies of their B and I forms and the fact that, experimentally, the $0 \rightarrow 0$ transition of the B form is also blue shifted (albeit by $0.1 \mathrm{eV}$ ) with respect to the I form.

To prepare this alternative B form, we appropriately twist Ser65 and Glu222, but keep the S65T-like orientation of Thr203 bonded to the phenolic oxygen for consistency with our standard B form and with the bulk of the literature (Thr203 is twisted away from the phenolic oxygen in Ref. 40). We then optimize a small cluster within DFT with fixed surrounding residues and, starting from this structure, perform a QM/MM simulation of 15 ps followed by annealing. During the 15 ps run, the hydrogen-bond network around the phenolic oxygen behaves similarly to what observed for the standard setup. In particular, the water molecule and Thr203 are constantly bonded to the phenolic oxygen but His148 and Tyr145 intermittently bond and detach. The final structure resembles the one of Ref. 40 but displays a hydrogen bond between His148 and the phenolic oxygen of the chromophore instead of having the water molecule bridging the histidine to the chromophore.

Finally, we compute the excitation energies on this structure at the level of CASPT2/MM, TDDFT/MM, and TDDFT on a cluster model. Disappointingly and in agreement with Ref. 40, we obtain a small blue shift of 0.04-0.07 in the excitation energies with respect to the annealed structure of our standard B form. Our results therefore suggest that the conformational changes of Ser65 and Glu222 do indeed have some effect on the excitation energy but in the opposite direction than what a simple analysis would have suggested. We stress however that we do not agree with Ref. 40 in assigning these structural changes to the difference between the I and $\mathrm{B}$ forms based on this small difference in excitation energies. The only available experimental data for the I form [112] is the $0 \rightarrow 0$ transition at room temperature and not the absorption maximum at low temperature, we should compare our vertical excitation energy for the annealed structure. Furthermore, we have shown above that temperature effects on the excitation energies are of this same order of magnitude. Finally, temperature effects were neglected altogether in Ref. 40, so their relative absolute energies for the A, B, and I forms should be compared to low-temperature populations. Consequently, since they estimated the A form to be $1 \mathrm{kcal} / \mathrm{mol}$ below the I and B forms, this would lead to the A form being exclusively populated at low temperature in contradiction with experiments at $1.6 \mathrm{~K}$ [112], which indicate that the $\mathrm{B}$ form is energetically favorable to the A form and the I form entirely unpopulated.

\subsection{Discussion and conclusions}

In this paper, we have thoroughly investigated the multi-scale computation of the excitation energies of wild-type GFP, examining the causes of the spectral spread and identifying the most suitable protocol for an accurate and affordable calculation of the absorption properties of this prototypical fluorescent protein.

Through extensive QM/MM molecular dynamics simulations, we explored the possible conformations of the protein in both the neutral A and anionic B forms, and found that the A form displays a very stable hydrogen-bond network, while 
the B form exhibits more significant deviations from the average structures even in the 20 ps time interval we examined. Analyzing 50 equidistant frames for each protonation form at the TDDFT/MM level, we found remarkably large spreads in the excitation energies of up to $0.5 \mathrm{eV}$ in both cases and, for the $\mathrm{B}$ form, confirmed the correctness of the TDDFT excitation-structure relation through CASPT2/MM calculations on the same 50 frames. Surprisingly, the structurally more stable A form has a larger standard deviation in the energies, which we find to be more sensitive to the internal coordinates of the chromophore, while the environment plays a bigger influence on the excitations of the B form. Including several frames, as done here, ensures a faithful sampling of the possible conformations of the chromophore and its surroundings.

A first screening of the excitation energies of these numerous frames with the cheap MM embedding yielded excitation energies blue shifted with respect to experiments. Depending on the quantum method, the excitation energies are in the range of 3.2-3.6 for the A form and 2.8-3.1 eV for the B form compared to an experimental absorption maximum of 3.1 and $2.6 \mathrm{eV}$, respectively. For both forms, CASPT2 gives the lowest excitation energies, QMC the highest ones, and CAM-B3LYP lies between the two, while NEVPT2, only employed on the B form, agrees reasonably well with QMC. Our findings are in line with other studies in literature on wild-type GFP [7, 9, 10,13] and other photosensitive bio-systems [8,11, 12, 119] which found blue-shifted excitation energies compared to experiments using different quantum methods in combination with an MM environment. Including temperature effects, as done here and in previous work $[11,12,19]$, fails to improve on experimental agreement.

While there is clearly a spread in the values stemming from the choice of excitedstate quantum method, the coarseness of the MM description is responsible for a systematic blue shift. To demonstrate this, we used 10 frames from cluster analysis for each protonation form and compared the MM results at the TDDFT level to calculations done on clusters containing almost 300 atoms, which we showed to be sufficiently large to account for most of the tuning by the protein environment. Within TDDFT, which is the only method allowing the treatment of such large clusters, the MM description causes a blue shift of about 0.03-0.19 eV when compared to the reference cluster calculations of both the A and the B forms. Even though the use of large clusters does not greatly ameliorate the agreement of TDDFT with experiments, the measure of success/failure of a particular description of the environment is its ability/inability to reproduce the excitation energies on large clusters treated at the same level of theory.

Given the evident inadequacy of static point charges, we explored two alternative approaches to improve the description of the environment. Remaining within classical methods, we investigated the inclusion of induced dipoles (MMpol) and performed TDDFT/MMpol in linear response (polLR) on ten frames for each protonation form. The cluster TDDFT excitation energies and the MMpol calculations on exactly the same cluster agree remarkably well for both the A and B forms. We note that, when comparing with previous studies with a similar MMpol methodology $[19,21]$, we find a good agreement for the B form but not for the A form, 
which displays a higher sensitivity in the excitation to the internal coordinates of the chromophore. As discussed in the SI, if one accounts for the use of a different DFT functional in optimizing the quantum chromophore (PBE in our case and B3LYP in those studies) and differences in the structural relaxation of the environment (fixed from a classical MD simulation in Ref. [19]), the discrepancy reduces to less than $0.1 \mathrm{eV}$ also for the A form. Importantly for our discussion, the shifts with respect to the MM values induced by their polarizable dipoles obtained in a LoProp construction [127] are in very good agreement with our findings. This shows that the more cost-effective choice of semi-empirical force-field parameters is adequate for the description of these systems as also apparent from the agreement of our TDDFT/MMpol results with the cluster calculations.

The good performance shown by the TDDFT/MMpol approach allows us to better understand the role of the environment on determining the excitation energies of the two forms of the GFP chromophore. By comparing three different formulations of the embedding model, namely, the ground-state (polGS), state-specific (polSS) and linear-response (polLR) flavors, it is apparent that, in GFP, the response of the environment to the excitation cannot be represented in terms of purely electrostatic effects induced by the change in the electronic density upon excitation: In fact, the polSS result which accounts for the electrostatic response of the environment to the excitation is almost equivalent to that obtained by assuming that the polarization of the environment is frozen in the configuration corresponding to the chromophore in its ground state (polGS). On the contrary, if we switch on the polLR interaction, a quite different picture is obtained as regards the nature of the chromophore-protein coupling. This interaction comes from the dynamical response of the environment (here represented by the induced dipoles) to the density of charge of the chromophore oscillating at the Bohr frequency (i.e., in a dipolar approximation, to the transition dipole). Such a "resonance" term, which might be classified as a part of dispersion, leads to a significant red-shift with respect to polGS (or the nonpolarizable MM scheme) and agreement with the reference cluster calculations is finally recovered.

In view of these findings, it is perhaps not so surprising that the other route we followed to improve over static MM charges, namely, sub-system DFT embedding was not successful: This scheme turned out to be at best equivalent to the MM description and, in the case of the anionic B form, to produce further unphysical blue shifts which persist across several quantum methods (CASPT2, QMC, and TDDFT) if the environment is relaxed through freeze\&thaw cycles. The recently introduced statespecific formulation of DFT embedding $[26,126]$ did not capture any response of the environment, and increasing the quantum DFT environment brought further blue shifts. While displaying a somewhat worse performance than the polGS and polSS formulations of MMpol probably due to the quality of the approximate kinetic functional, the behavior of DFT embedding is in line with the MMpol results and reveals that the state-specific formulation of sub-system DFT can recover electrostatic effects but that these are not dominant in this system. Similarly, one expects that other alternative DFT-based embedding techniques that eliminate the need for kinetic-energy functionals either by reconstructing the embedding potentials [128] or imposing orthogonality of the orbitals of the sub-systems [129] will not possess the necessary 


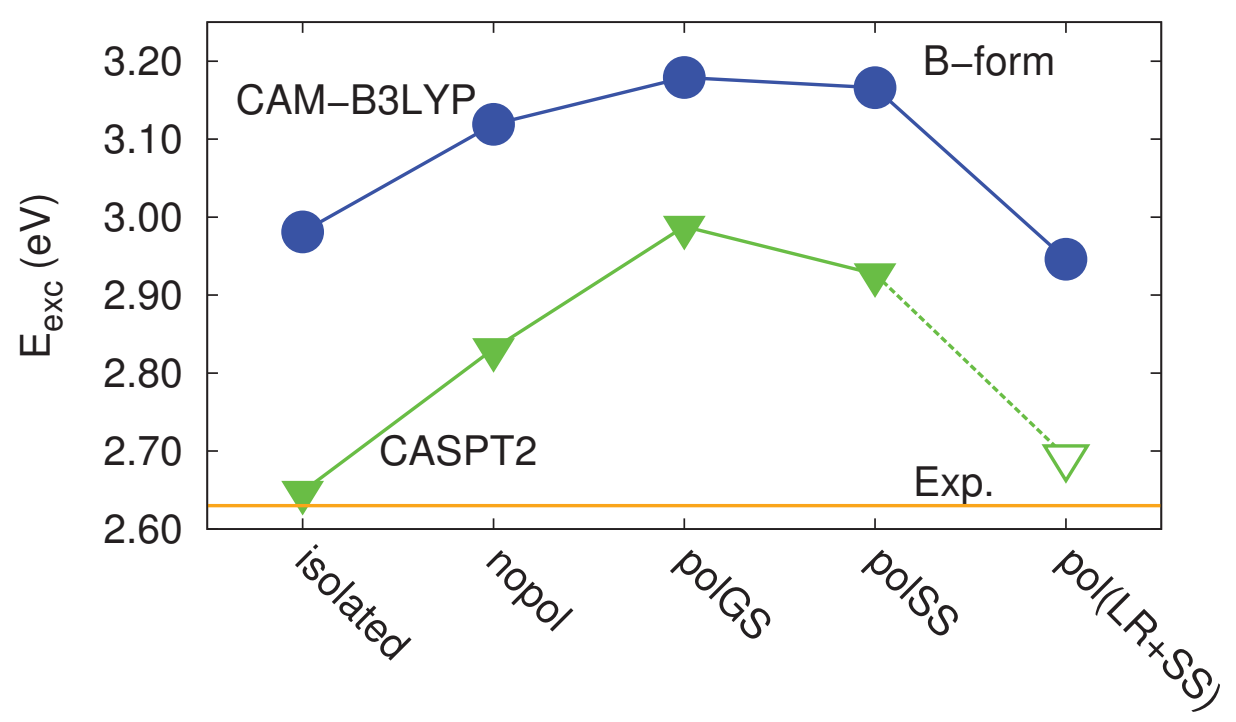

Figure 5.14: B form: Excitation energies computed with CASPT2 on the chromophore only (isolated) or surrounded by the environment described with static point charges (nopol), with point charges and dipoles polarized within CASSCF/MMpol in the ground state (polGS), in the excited state (polSS) or using an estimate based on the linear-response (polLR) TDDFT result (see text). The calculations are performed on the annealed frame.

ingredients to describe the excited states of GFP: They will improve the ground-state description of the environment and, even if they included state-specificity, will not easily capture the coupling with the environment beyond electrostatic effects.

While the MMpol embedding scheme has allowed us to identify the necessary ingredients in the description of the excited states of GFP, we have been able to include either the state-specific (electrostatic) or the linear-response (resonance) polarization of the environment but not both in a united formulation without resorting to a complete "brute-force" quantum calculation on a large quantum cluster. Furthermore, we have done so only at the TDDFT level. Since both the SS and the LR responses depend on the quantum method used to describe the excitation, what can we achieve with a correlated method considering that a large quantum cluster is out of reach and an LR formulation often not available? In an effort towards a more complete and accurate simulation of the excitation process in GFP, we attempt here to estimate the best possible excitation values using a CASPT2 description of the excitation process instead of TDDFT. At the CASPT2 level, one can evaluate the excitation energy in a polSS scheme using the state-specific induced dipoles obtained within CASSCF/MMPol in the computation of the CASPT2 energies (see Computational Details). Then, we estimate the polLR correction as the TDDFT/MMpol (polLR) shift with respect to the corresponding polGS value and rescaling it with the ratio squared of the CASPT2 and TDDFT transition dipole moments.

The final picture emerging from this approximate CASPT2 estimate of the effects of the environment is illustrated for the B form in Figure 5.14 where the different 
CASPT2 excitation energies computed with static point charges, ground-state and state-specific induced dipoles are compared to the extrapolated polLR+polSS value. As expected from the results in the previous section, the response at the correlated level is stronger than in TDDFT when the MM description is added and then enhanced with induced dipoles in a polGS and, subsequently, polSS formulation. The estimated polLR correction is instead rather similar to the TDDFT shift since the ratio of the CASPT2 and TDDFT transition dipole moments is in this case very close to one. That the extrapolated CASPT2 energy correlates very well with experiments is an appealing result, which should however not be overstated. One should apply a similar analysis to the other correlated methods used in this study, which further blueshift the excitation energy with respect to CASPT2 but also respond more strongly to changes in the description of the environment (a perfect agreement between vertical excitation energies and absorption maximum is anyhow a misguided expectation also for the fluorescent B form). However, our extrapolation clearly shows that environmental effects on the excitation processes in GFP are the result of different interactions in the ground and the excited state; only by adopting a polarizable approach which can account for both state-specific relaxation and "resonance" coupling we can get a semiquantitatively correct description. 


\section{Bibliography}

[1] H. M. Senn and W. Thiel, Ang. Chem. Int. Ed. 48, 1198 (2009).

[2] J. Neugebauer, Phys. Rep. 489, 1 (2010).

[3] B. Mennucci, Phys. Chem. Chem. Phys. 15, 6583 (2013).

[4] M. Karplus, Ang. Chem. Int. Ed. 53, 9992 (2014).

[5] M. Levitt, Ang. Chem. Int. Ed. 53, 10006 (2014).

[6] A. Warshel, Ang. Chem. Int. Ed. 53, 10020 (2014).

[7] R. Send, V. R. I. Kaila, and D. Sundholm, J. Chem. Phys. 134, 214114 (2011).

[8] C. M. Isborn, A. W. Götz, M. A. Clark, R. C. Walker, and T. J. Martinez, J. Chem. Theory Comput. 8, 5092 (2012).

[9] C. Filippi, F. Buda, L. Guidoni, and A. Sinicropi, J. Chem. Theory Comput. 8, 112 (2012).

[10] V. R. I. Kaila, R. Send, and D. Sundholm, Phys. Chem. Chem. Phys. 15, 4491 (2013).

[11] O. Valsson, P. Campomanes, I. Tavernelli, U. Rothlisberger, and C. Filippi, J. Chem. Theory Comput. 9, 2441 (2013).

[12] P. Amat and R. Nifosi, J. Chem. Theory Comput. 9, 497 (2013).

[13] T. Schwabe, M. T. Beerepoot, J. M. Olsen, and J. Kongsted, Phys. Chem. Chem. Phys. 17, 2582 (2015).

[14] M. A. Thomspon, J. Phys. Chem. 100, 14492 (1996).

[15] C. Curutchet, A. Muñoz-Losa, S. Monti, J. Kongsted, G. D. Scholes, and B. Mennucci, J. Chem. Theory Comput. 5, 1838 (2009).

[16] J. M. Olsen, K. Aidas, and J. Kongsted, J. Chem. Theory Comput. 6, 3721 (2010).

[17] L. V. Slipchenko, J. Phys. Chem. A 114, 8824 (2010). 
[18] A. H. Steindal, J. M. Olsen, K. Ruud, L. Frediani, and J. Kongsted, Phys. Chem. Chem. Phys. 14, 5440 (2012).

[19] M. T. Beerepoot, A. H. Steindal, J. Kongsted, B. O. Brandsdal, L. Frediani, K. Ruud, and J. M. Olsen, Phys. Chem. Chem. Phys. 15, 4735 (2013).

[20] M. T. Beerepoot, A. H. Steindal, K. Ruud, J. M. Olsen, and J. Kongsted, Comp. Theor. Chem. 1040-1041, 304 (2014).

[21] A. Pikulska, A. H. Steindal, M. T. P. Beerepoot, and M. Pecul, J. Phys. Chem. B 119, 3377 (2015), pMID: 25646666.

[22] K. Sneskov, T. Schwabe, O. Christiansen, and J. Kongsted, Phys. Chem. Chem. Phys. 13, 18551 (2011).

[23] M. Svensson, S. Humbel, R. D. J. Froese, T. Matsubara, S. Sieber, and K. Morokuma, J. Phys. Chem. 100, 19357 (1996).

[24] A. S. Pereira Gomes, C. R. Jacob, and L. Visscher, Phys. Chem. Chem. Phys. 10, 5353 (2008).

[25] A. S. P. Gomes and C. R. Jacob, Annu. Rep. Prog. Chem., Sect. C 108, 222 (2012).

[26] C. Daday, C. König, O. Valsson, J. Neugebauer, and C. Filippi, J. Chem. Theory Comput. 9, 2355 (2013).

[27] R. Send, C.-M. Suomivuori, V. R. I. Kaila, and D. Sundholm, J. Phys. Chem. B 119, 2933 (2015).

[28] R. N. Day and M. W. Davidson, Chem. Soc. Rev. 38, 2887 (2009).

[29] R. H. Newman, M. D. Fosbrink, and J. Zhang, Chem. Rev. 111, 3614 (2011).

[30] S. W. Hell, Science 316, 1153 (2007).

[31] G. H. Patterson, Semin. Cell Dev. Biol. 20, 886 (2009).

[32] J.-Y. Hasegawa, K. Fujimoto, B. Swerts, T. Miyahara, and H. Nakatsuji, J. Comput. Chem. 28, 2443 (2007).

[33] M. A. L. Marques, X. López, D. Varsano, A. Castro, and A. Rubio, Phys. Rev. Lett. 90, 258101 (2003).

[34] T. Laino, R. Nifosi, and V. Tozzini, Chem. Phys. 298, 17 (2004).

[35] A. Sinicropi, T. Andruniow, N. Ferré, R. Basosi, and M. Olivucci, J. Am. Chem. Soc. 127, 11534 (2005).

[36] K. B. Bravaya, M. G. Khrenova, B. L. Grigorenko, A. V. Nemukhin, and A. I. Krylov, J. Phys. Chem. B 115, 8296 (2011). 
[37] K. B. Bravaya, B. L. Grigorenko, A. V. Nemukhin, and A. I. Krylov, Acc. Chem. Res. 45, 265 (2011).

[38] B. L. Grigorenko, A. V. Nemukhin, D. I. Morozov, I. Polyakov, K. B. Bravaya, and A. I. Krylov, J. Chem. Theory Comput. 8, 1912 (2012).

[39] A. Petrone, P. Caruso, S. Tenuta, and N. Rega, Phys. Chem. Chem. Phys. 15, 20536 (2013).

[40] B. L. Grigorenko, A. V. Nemukhin, I. Polyakov, D. I. Morozov, and A. I. Krylov, J. Am. Chem. Soc. 135, 11541 (2013).

[41] A. Laio, J. VandeVondele, and U. Röthlisberger, J. Chem. Phys. 116, 6941 (2002).

[42] A. Laio, J. VandeVondele, and U. Röthlisberger, J. Phys. Chem. B 106, 7300 (2002).

[43] CPMD v3.13.1,, copyright IBM Corp, 1990-2008; Copyright MPI für Festkörperforschung Stuttgart, 1997-2001; http://www.cpmd.org/.

[44] W. F. van Gunsteren, S. R. Billeter, A. A. Eising, P. H. Huenenberger, P. Krueger, A. E. Mark, W. R. P. Scott, and I. G. Tironi, Biomolecular Simulation: The Gromos96 Manual and User Guide, Zurich, 1996, hochschulverlag an der ETH Zurich.

[45] Y. Duan, C. Wu, S. Chowdhury, M. C. Lee, G. Xiong, W. Zhang, R. Yang, P. Cieplak, R. Luo, T. Lee, J. Caldwell, J. Wang, and P. Kollman, J. Comput. Chem. 24, 1999 (2003).

[46] W. L. Jorgensen, J. Chandrasekhar, J. D. Madura, R. W. Impey, and M. L. Klein, J. Chem. Phys. 79, 926 (1983).

[47] J. P. Perdew, K. Burke, and M. Ernzerhof, Phys. Rev. Lett. 77, 3865 (1996).

[48] J. P. Perdew, K. Burke, and M. Ernzerhof, Phys. Rev. Lett. 78, 1396 (1997).

[49] N. Troullier and J. L. Martins, Phys. Rev. B 43, 1993 (1991).

[50] H. J. C. Berendsen, J. P. M. Postma, W. F. van Gunsteren, A. DiNola, and J. R. Haak, J. Chem. Phys. 81, 3684 (1984).

[51] S. Nosé, J. Chem. Phys. 81, 511 (1984).

[52] W. G. Hoover, Phys. Rev. A 31, 1695 (1985).

[53] F. Aquilante, L. D. Vico, N. Ferré, G. Ghigo, P.-Å. Malmqvist, P. Neogrády, T. B. Pedersen, M. Pitonák, M. Reiher, B. O. Roos, L. Serrano-Andrés, M. Urban, V. Veryazov, and R. Lindh, J. Comp. Chem. 31, 224 (2010). 
[54] P. Huang and E. A. Carter, J. Chem. Phys. 125, 084102 (2006).

[55] S. Sharifzadeh, P. Huang, and E. A. Carter, Chem. Phys. Lett. 470, 347 (2009).

[56] P.-O. Widmark, P.-Å. Malmqvist, and B. O. Roos, Theor. Chem. Acc. 77, 291 (1990).

[57] P. Widmark, P. Malmqvist, and B. O. Roos, Theor. Chem. Acc. 77, 291 (1990).

[58] T. H. Dunning Jr, J. Chem. Phys. 90, 1007 (1989).

[59] F. Aquilante, P.-Å. Malmqvist, T. B. Pedersen, A. Ghosh, and B. O. Roos, J. Chem. Theory Comput. 4, 694 (2008).

[60] G. Ghigo, B. O. Roos, and P.-Å. Malmqvist, Chem. Phys. Lett. 396, 142 (2004).

[61] N. Forsberg and P.-A. Malmqvist, Chem. Phys. Lett. 274, 196 (1997).

[62] J. Wang, P. Cieplak, and P. A. Kollman, J. Comput. Chem. 21, 1049 (2000).

[63] J. Wang, P. Cieplak, J. Li, T. Hou, L. Ray, and D. Yong, J. Chem. Phys. B 8, 3091 (2011).

[64] J. Wang, P. Cieplak, J. Li, J. Wang, Q. Cai, M. Hsieh, H. Lei, R. Luo, and Y. Duan, J. Chem. Phys. B 8, 3100 (2011).

[65] Q. Li, B. Mennucci, M. A. Robb, L. Blancafort, and C. Curutchet, J. Chem. Theory Comput. 11, 1674 (2015).

[66] M. J. Frisch, G. W. Trucks, H. B. Schlegel, G. E. Scuseria, M. A. Robb, J. R. Cheeseman, G. Scalmani, V. Barone, B. Mennucci, G. A. Petersson, H. Nakatsuji, M. Caricato, X. Li, H. P. Hratchian, A. F. Izmaylov, J. Bloino, G. Zheng, J. L. Sonnenberg, M. Hada, M. Ehara, K. Toyota, R. Fukuda, J. Hasegawa, M. Ishida, T. Nakajima, Y. Honda, O. Kitao, H. Nakai, T. Vreven, J. J. A. Montgomery, J. E. Peralta, F. Ogliaro, M. Bearpark, J. J. Heyd, E. Brothers, K. N. Kudin, V. N. Staroverov, R. Kobayashi, J. Normand, K. Raghavachari, A. Rendell, J. C. Burant, S. S. Iyengar, J. Tomasi, M. Cossi, N. Rega, J. M. Millam, M. Klene, J. E. Knox, J. B. Cross, V. Bakken, C. Adamo, J. Jaramillo, R. Gomperts, R. E. Stratmann, O. Yazyev, A. J. Austin, R. Cammi, C. Pomelli, J. W. Ochterski, R. L. Martin, K. Morokuma, V. G. Zakrzewski, G. A. Voth, P. Salvador, J. J. Dannenberg, S. Dapprich, A. D. Daniels, Ö. Farkas, J. B. Foresman, J. V. Ortiz, J. Cioslowski, and D. J. Fox, Gaussian 09 Revision A.2, gaussian 09 Revision A.2, Gaussian Inc. Wallingford CT 2009.

[67] T. Yanai, D. P. Tew, and N. C. Handy, Chem. Phys. Lett. 393, 51 (2004).

[68] H. Iikura, T. Tsuneda, T. Yanai, and K. Hirao, J. Chem. Phys. 115, 3540 (2001). 
[69] W. J. Hehre, R. Ditchfield, and J. A. Pople, J. Chem. Phys. 56, 2257 (1972).

[70] T. Clark, J. Chandrasekhar, G. W. Spitznagel, and P. v. R. Schleyer, J. Comput. Chem. 4, 294 (1983).

[71] M. J. Frisch, J. A. Pople, and J. S. Binkley, J. Chem. Phys. 80, 3265 (1984).

[72] G. te Velde, F. M. Bickelhaupt, E. J. Baerends, S. J. A. van Gisbergen, C. Fonseca Guerra, J. G. Snijders, and T. Ziegler, J. Comput. Chem. 22, 931 (2001).

[73] C. Fonseca Guerra, J. G. Snijders, G. te Velde, and E. J. Baerends, Theor. Chem. Acc. 99, 391 (1998).

[74] ADF2013, SCM, Theoretical Chemistry, Vrije Universiteit, Amsterdam, The Netherlands, http://www.scm.com (access date: 06/06/2014).

[75] E. V. Lenthe and E. J. Baerends, J. Comput. Chem. 24, 1142 (2003).

[76] Y. Zhao and D. G. Truhlar, J. Phys. Chem. A 110, 13126 (2006).

[77] A. Lembarki and H. Chermette, Phys. Rev. A 50, 5328 (1994).

[78] J. P. Perdew, Phys. Lett. A 165, 79 (1992).

[79] Y. Akinaga and S. Ten-no, Chem. Phys. Lett. 462, 348 (2008).

[80] M. Seth and T. Ziegler, J. Chem. Theory Comput. 8, 901 (2012).

[81] C. Angeli, R. Cimiraglia, S. Evangelisti, T. Leininger, and J.-P. Malrieu, J. Chem. Phys. 114, 10252 (2001).

[82] C. Angeli, R. Cimiraglia, and J.-P. Malrieu, Chem. Phys. Lett. 350, 297 (2001).

[83] C. Angeli, R. Cimiraglia, and J.-P. Malrieu, J. Chem. Phys. 117, 9138 (2002).

[84] N. F., Wiley Interdiscip. Rev.: Comput. Mol. Sci. 2, 73 (2012).

[85] K. Eichkorn, O. Treutler, H. Öhm, M. Hada, M. Häser, and R. Ahlrichs, Chem. Phys. Lett. 240, 283 (1995).

[86] F. Neese, F. Wennmohs, A. Hansen, and U. Becker, Chem. Phys. 356, 98 (2009).

[87] K. G. Dyall, J. Chem. Phys. 102, 4909 (1995).

[88] C. Angeli, R. Cimiraglia, and J.-P. Malrieu, Chem. Phys. Lett. 317, 472 (2000).

[89] CHAMP is a quantum Monte Carlo program package written by C. J. Umrigar, C. Filippi and collaborators. 
[90] M. Burkatzki, C. Filippi, and M. Dolg, J. Chem. Phys. 126, 234105 (2007).

[91] For the hydrogen atom, we use a more accurate BFD pseudopotential and basis set. Dolg, M.; Filippi, C., private communication.

[92] We add one $s$ and one $p$ diffuse function on the carbon and the nitrogen using exponents from the aug-cc-pVDZ basis set, taken from EMSL Basis Set Library (http://bse.pnl.gov).

[93] C. Filippi and C. J. Umrigar, J. Chem. Phys. 105, 213 (1996), as Jastrow correlation factor, we use the exponential of the sum of three fifth-order polynomials of the electron-nuclear (e-n), the electron-electron (e-e). The Jastrow factor is adapted to deal with pseudo-atoms, and the scaling factor $\kappa$ is set to 0.6 a.u. The 2-body Jastrow factor includes five parameters in the e-e terms and four parameters for each atom type in the e-n terms.

[94] C. J. Umrigar, J. Toulouse, C. Filippi, S. Sorella, and R. G. Hennig, Phys. Rev. Lett. 98, 110201 (2007).

[95] C. Filippi, M. Zaccheddu, and F. Buda, J. Chem. Theory Comput. 5, 2074 (2009).

[96] M. Casula, Phys. Rev. B 74, 161102 (2006).

[97] J. C. Phillips, R. Braun, W. Wang, J. Gumbart, E. Tajkhorshid, E. Villa, C. Chipot, R. D. Skeel, L. Kalé, and K. Schulten, J. Comput. Chem. 26, 1781 (2005).

[98] L. J. Heyer, S. Kruglyak, and S. Yooseph, Genome Res. 9, 1106 (1999).

[99] W. Humphrey, A. Dalke, and K. Schulten, J. Mol. Graphics 14, 33 (1996).

[100] M. Chattoraj, B. A. King, G. U. Bublitz, and S. G. Boxer, Proc. Natl. Acad. Sci. 93, 8362 (1996).

[101] K. Brejc, T. K. Sixma, P. A. Kitts, S. R. Kain, R. Y. Tsien, M. Ormö, and S. J. Remington, Proc. Natl. Acad. Sci. 94, 2306 (1997).

[102] D. Stoner-Ma, A. A. Jaye, P. Matousek, M. Towrie, S. R. Meech, and P. J. Tonge, J. Am. Chem. Soc. 127, 2864 (2005).

[103] R. Nifosi and V. Tozzini, Proteins: Struct., Funct., Genet. 51, 378 (2003).

[104] M. Ormo, A. B. Cubitt, K. Kallio, L. A. Gross, R. Y. Tsien, and S. J. Remington, Science 273, 1392 (1996).

[105] M. Elsliger, R. Wachter, G. Hanson, K. Kallio, and S. Remington, Biochemistry 38, 5296 (1999).

[106] R. K. Jain and R. Ranganathan, Proc. Natl. Acad. Sci. 101, 111 (2004). 
[107] W. W. Ward, C. W. Cody, R. C. Hart, and M. J. Cormier, Photochem. Photobiol. 31, 611 (1980).

[108] H. Niwa, S. Inouye, T. Hirano, T. Matsuno, S. Kojima, M. Kubota, M. Ohashi, and F. I. Tsuji, Proc. Natl. Acad. Sci. 93, 13617 (1996).

[109] N. M. Webber, K. L. Litvinenko, and S. R. Meech, J. Phys. Chem. B 105, 8036 (2001).

[110] K. L. Litvinenko, N. M. Webber, and S. R. Meech, Chem. Phys. Lett. 346, 47 (2001).

[111] D. Mandal, T. Tahara, N. M. Webber, and S. R. Meech, Chem. Phys. Lett. 358, 495 (2002).

[112] T. M. H. Creemers, A. J. Lock, V. Subramaniam, T. M. Jovin, and S. Völker, Nat. Struct. Biol. 6, 557 (1999).

[113] R. Kobayashi and R. D. Amos, Chem. Phys. Lett. 420, 106 (2006).

[114] Z.-L. Cai, M. J. Crossley, J. R. Reimers, R. Kobayashi, and R. D. Amos, J. Phys. Chem. B 110, 15624 (2006).

[115] D. J. Tozer, R. D. Amos, N. C. Handy, B. O. Roos, and L. Serrano-ANDRES, Mol. Phys. 97, 859 (1999).

[116] A. Dreuw and M. Head-Gordon, J. Am. Chem. Soc. 126, 4007 (2004), pMID: 15038755 .

[117] M. Wanko, P. Garcia-Risueño, and A. Rubio, Phys. Status Solidi B 392 (2012).

[118] P. Armengol, R. Gelabert, M. Moreno, and J. M. Lluch, Org. Biomol. Chem. 12, 9845 (2014).

[119] M. Wanko, M. Hoffmann, T. Frauenheim, and M. Elstner, J. Phys. Chem. B 112, 11462 (2008).

[120] M. Caricato, B. Mennucci, J. Tomasi, F. Ingrosso, R. Cammi, S. Corni, and G. Scalmani, J. Chem. Phys. 124, 124520 (2006).

[121] S. Corni, R. Cammi, B. Mennucci, and J. Tomasi, J. Chem. Phys. 123, 134512 (2005).

[122] R. Cammi, S. Corni, B. Mennucci, and J. Tomasi, J Chem Phys 122, 104513 (2005).

[123] T. A. Wesolowski and A. Warshel, J. Phys. Chem. 97, 8050 (1993).

[124] M. Humbert-Droz, X. Zhou, S. V. Shedge, and T. A. Wesolowski, Theor. Chem. Acc. 133, 1405 (2014). 
[125] T. A. Wesolowski and J. Weber, Chem. Phys. Lett. 248, 71 (1996).

[126] C. Daday, C. König, J. Neugebauer, and C. Filippi, ChemPhysChem 15, 3205 (2014).

[127] L. Gagliardi, R. Lindh, and G. Karström, J. Chem. Phys. 121, 4494 (2004).

[128] S. Fux, C. R. Jacob, J. Neugebauer, L. Visscher, and M. Reiher, J. Chem. Phys. 132, (2010).

[129] F. R. Manby, M. Stella, J. D. Goodpaster, and T. F. Miller, J. Chem. Theory Comput. 8, 2564 (2012), pMID: 22904692. 


\section{Chapter 6}

\section{Wavefunction embedding using exact Freeze\&Thaw cycles ${ }^{\dagger}$}

Given the slightly disappointing performance of subsystem DFT embedding in Chapter 5, we explore here an alternative way of conducting embedding calculations: We employ a scheme based on reconstructed potentials, thereby eliminating the need of a kinetic-energy functional, which has been established as one of the main approximations in the procedure of Chapter 4 . We show that using these exact kinetic-energy potentials obtained via reconstruction, we can reproduce supermolecular densities by only performing subsystem calculations, thereby proving the exact nature of this approach. As a practical route to reconstruction, we test two closely related procedures: The $\mathrm{Wu}$-Yang scheme and the so-called virtual projection approach, which removes the unphysical dependence of the final potential on some computational parameters of the reconstruction procedure. We also perform excited-state wave function calculations in the embedding potentials and demonstrate the accuracy of the potentials obtained with the virtual projection procedure.

\subsection{Theory}

\subsubsection{Reconstruction techniques}

In a DFT calculation, one solves the non-interacting Kohn-Sham (KS) equations self consistently:

$$
\left(-\frac{1}{2} \nabla^{2}+v_{s}(\mathbf{r})\right) \phi_{i}(\mathbf{r})=\epsilon_{i} \phi_{i}(\mathbf{r})
$$

which yield the ground-state density of the interacting system as

$$
\rho(\mathbf{r})=\sum_{i}\left|\phi_{i}(\mathbf{r})\right|^{2}
$$

$\dagger$ This chapter is in preparation to be published as C. Daday, C. Filippi, A. S. P. Gomes, and C. Jacob, "Wavefunction embedding using exact Freeze\&Thaw cycles", 
The one-body KS potential is given by

$$
v_{s}(\mathbf{r})=v_{\mathrm{nuc}}(\mathbf{r})+\int \frac{\rho\left(\mathbf{r}^{\prime}\right)}{\left|\mathbf{r}-\mathbf{r}^{\prime}\right|} \mathrm{d} \mathbf{r}+\frac{\delta E_{\mathrm{xc}}[\rho]}{\delta \rho(\mathbf{r})},
$$

where $E_{\mathrm{xc}}(\rho)$ is the exchange-correlation functional. The solution of the KS problem will minimize the energy functional:

$$
E[\rho]=T_{s}[\rho]+\int v_{\text {nuc }}(\mathbf{r}) \rho(\mathbf{r}) \mathrm{d} \mathbf{r}+\frac{1}{2} \int \frac{\rho(\mathbf{r}) \rho\left(\mathbf{r}^{\prime}\right)}{\left|\mathbf{r}-\mathbf{r}^{\prime}\right|} \mathrm{d} \mathbf{r} \mathrm{d} \mathbf{r}^{\prime}+E_{\mathrm{xc}}[\rho],
$$

where the noninteracting kinetic energy is known exactly as a function of the orbitals:

$$
T_{s}[\rho]=-\frac{1}{2} \sum_{i}\left\langle\phi_{i}\left|\nabla^{2}\right| \phi_{i}\right\rangle .
$$

The only approximation in a KS calculation enters in the exchange-correlation functional $E_{\mathrm{xc}}[\rho]$ since the electrostatic terms and the kinetic energy can be computed exactly. However, in frozen-density embedding (FDE) extensively discussed in Chapters 3, 4, and 5, one also needs the expression of the noninteracting kinetic energy as a functional of the density, that is, without the benefit of knowing the orbitals of the system. In particular, the functional derivative of the noninteracting kinetic energy enters in the definition of the embedding potential of a given subsystem, so one needs an approximate noninteracting kinetic-energy density functional (KEDF) in addition to the exchange-correlation one.

Minimization of Eq. 6.4 with respect to the density (with the constraint that the density integrates to the given number of electrons) yields a relation between the KS potential $v_{s}$ and the functional derivative of the kinetic energy:

$$
v_{t}[\rho](\mathbf{r})=\frac{\delta T_{s}[\rho]}{\delta \rho(\mathbf{r})}=\mu-v_{s}[\rho]
$$

where $\mu$ is a constant shift (the chemical potential). Therefore, if, for a given density, we can determine the $\mathrm{KS}$ potential $v_{s}$ with yields that density as the non-interacting ground-state density, we can determine the functional derivative of the kinetic energy computed at the same density [1]. Since the kinetic potential is the term needed in an embedding calculation, we will exploit this equation in order to eliminate the need of an approximate KEDF, as will be shown below.

The problem of determining the kinetic-energy potential is therefore equivalent to one of computing the potential $v_{s}$. It is possible to determine a potential $\tilde{v}_{s}$ that, in a KS calculation, yields a given ground-state target density $\rho^{\text {target }}$ through a socalled reconstruction procedure (in the following, $\tilde{v}_{s}$ always refers to the result of a reconstruction). In practice, for the given target density, we define convergence in such a reconstruction scheme in terms of a density-error threshold:

$$
\int\left|\rho\left[\tilde{v}_{s}\right](\mathbf{r})-\rho^{\operatorname{target}}(\mathbf{r})\right| \mathrm{d} \mathbf{r}<e_{\mathrm{dens}}
$$


where by $\rho\left[\tilde{v}_{s}\right]$ we denote the ground-state density computed in a KS calculation using the reconstructed potential $\tilde{v}_{s}$.

In the Wu-Yang [2-5] (WY) reconstruction scheme or direct optimization, the potential is computed by maximizing the following Lagrangian with respect to it:

$$
W_{s}\left[\tilde{v}_{s}\right]=-\frac{1}{2} \sum_{i}\left\langle\phi_{i}\left|\nabla^{2}\right| \phi_{i}\right\rangle+\int \tilde{v}_{s}(\mathbf{r})\left(\rho\left[\tilde{v}_{s}\right](\mathbf{r})-\rho^{\operatorname{target}}(\mathbf{r})\right) \mathrm{d} \mathbf{r} .
$$

where the reconstructed potential will be given as the sum of a "guess" potential and a linear combination of potential basis functions whose coefficients we want to determine:

$$
\tilde{v}_{s}(\mathbf{r})=v_{s}^{\text {guess }}(\mathbf{r})+\sum_{t} b_{t} g_{t}(\mathbf{r}) .
$$

As initial guess in our procedure, we choose the following potential:

$$
v_{s}^{\text {guess }}(\mathbf{r})=v_{\text {nuc }}(\mathbf{r})+\int \frac{\rho\left(\mathbf{r}^{\prime}\right)}{\left|\mathbf{r}-\mathbf{r}^{\prime}\right|} \mathrm{d} \mathbf{r}+v_{0}(\mathbf{r}),
$$

where $v_{0}$ is an approximate exchange-correlation potential, in our case, the FermiAmaldi potential [6]:

$$
v_{0}(\mathbf{r})=-\frac{\zeta}{N} \int \frac{\rho\left(\mathbf{r}^{\prime}\right)}{\left|\mathbf{r}-\mathbf{r}^{\prime}\right|} \mathrm{d} \mathbf{r}^{\prime}
$$

with $\zeta$ the exponent of the most diffuse Slater function in our orbital basis set. This expression satisfies the exact long-distance limit corresponding to the target density obtained with the given orbital basis set (decaying as the most diffuse function), and therefore the difference between the exact $v_{s}$ and the starting potential $v_{s}^{\text {guess }}$ is known to vanish at long distances.

It is possible to show that any reconstruction procedure using a finite orbital basis set suffers from the ill-posed nature of the problem: Several different embedding potentials can yield the same Kohn-Sham density due to the limitations of the orbital basis (the Hohenberg-Kohn theorem only applies in the basis-set limit). Therefore, there is a certain arbitrariness involved in any practical reconstruction scheme and changing certain numerical parameters such as the density threshold $e_{\text {dens }}$ or the starting potential can have strong effects on the final reconstructed potential. In practice, this has as a consequence that the $\mathrm{Wu}$-Yang reconstruction produces unphysical oscillations in the resulting potential, which depend strongly on the particular orbital basis and numerical parameters of the reconstruction.

There are several ways to address these issues. For example, one may impose a certain penalty function in the Lagrangian (Eq. 6.8) for oscillations of the potential [7]:

$$
\begin{aligned}
W_{s}^{\text {smooth }, \lambda}\left[\tilde{v}_{s}\right] & =-\frac{1}{2} \sum_{i}\left\langle\phi_{i}\left|\nabla^{2}\right| \phi_{i}\right\rangle \\
& +\int\left\{\tilde{v}_{s}[\rho](\mathbf{r})\left(\rho\left[\tilde{v}_{s}\right](\mathbf{r})-\rho^{\operatorname{target}}(\mathbf{r})\right)+\lambda\left|\nabla \tilde{v}_{s}(\mathbf{r})\right|^{2}\right\} \mathrm{d} \mathbf{r}
\end{aligned}
$$


which alleviates the unphysical oscillations but introduces another arbitrary parameter along with the density threshold, namely, the constant $\lambda$ of the penalty function. ${ }^{1}$ A more recent proposal is the so-called virtual projection [5], which determines a single potential that would yield approximately the same density independently of any expansion of the orbital basis set. Consequently, unphysical oscillations due to the finiteness of the basis set are removed and the potential is largely decoupled from the details of the reconstruction procedure. In general, the density error will increase due to the virtual projection but the potential is more physically meaningful. Therefore, since we want to employ the potential in a different quantum method as in a wave function in DFT embedding calculation, the potential obtained in the virtual projection scheme will be more accurate and robust than the original Wu-Yang potential that is guaranteed to give good results only for the particular basis set and method used in its construction (Kohn-Sham DFT). In particular, the effects of the rapid oscillations on the virtual orbitals will be unpredictable (the virtual orbitals being irrelevant in the ground-state reconstruction), making an excited-state calculation in such a potential possibly problematic. For the sake of comparison, we will employ here both the original Wu-Yang as well as the virtual projection method.

\subsubsection{Conventional DFT embedding}

As already discussed in Subsection 2.3.3, in a conventional subsystem DFT or wave function in DFT (WF/DFT) embedding calculation, the embedding potential has the following expression:

$$
\begin{aligned}
v_{\text {emb }, 1}\left[\rho_{1}, \rho_{2}\right](\mathbf{r}) & =v_{\text {nuc } 2}(\mathbf{r})+\int \frac{\rho_{2}\left(\mathbf{r}^{\prime}\right)}{\left|\mathbf{r}-\mathbf{r}^{\prime}\right|} \mathrm{d} \mathbf{r}^{\prime} \\
& +v_{\text {xc }}^{\text {nad }}\left[\rho_{1}, \rho_{2}\right](\mathbf{r})+v_{t}^{\text {nad }}\left[\rho_{1}, \rho_{2}\right](\mathbf{r}),
\end{aligned}
$$

where the nonadditive potentials are given by:

$$
\begin{aligned}
& v_{\mathrm{xc}}^{\operatorname{nad}}\left[\rho_{1}, \rho_{2}\right](\mathbf{r})=\left.\frac{\delta E_{\mathrm{xc}}[\rho]}{\delta \rho(\mathbf{r})}\right|_{\rho=\rho_{1}+\rho_{2}}-\left.\frac{\delta E_{\mathrm{xc}}[\rho]}{\delta \rho(\mathbf{r})}\right|_{\rho=\rho_{1}} \\
& v_{\mathrm{t}}^{\operatorname{nad}}\left[\rho_{1}, \rho_{2}\right](\mathbf{r})=\left.\frac{\delta T_{\mathrm{s}}[\rho]}{\delta \rho(\mathbf{r})}\right|_{\rho=\rho_{1}+\rho_{2}}-\left.\frac{\delta T_{\mathrm{s}}[\rho]}{\delta \rho(\mathbf{r})}\right|_{\rho=\rho_{1}},
\end{aligned}
$$

where $E_{\mathrm{xc}}[\rho]$ and $T_{\mathrm{s}}[\rho]$ must both be approximated with orbital-free functionals. If the exchange-correlation functional in our calculation is not explicitly dependent on the orbitals (i.e., an LDA or GGA), we can use the same functional in the nonadditive exchange-correlation potential and in the calculation to generate the density for the two subsystems, and the only additional approximation in comparison to a supermolecular Kohn-Sham calculation is the presence of an approximate kineticenergy density functional (KEDF). Eliminating the need for an approximate KEDF

\footnotetext{
${ }^{1}$ In practice, one may look for a suitable $\lambda$ that will increase the density error from the initial threshold $e_{\mathrm{dens}}$ to another, higher $e_{\mathrm{dens}}^{\text {smooth }}$ [4], but this modified approach will also require two parameters instead of one.
} 
is therefore a very desirable goal which would allow significant improvements on the performance of the embedding calculations.

We note that another method which avoids the use of a KEDF is the recently proposed projection-based scheme [8] in which the non-additive kinetic energy term is enforced to be zero by imposing orthogonality between the orbitals of the subsystems with the use of a nonlocal projector operator. Differently from the scheme of Ref. 8, we maintain here a local embedding potential which allows interfacing to a wider range of methods in case of WF/DFT and do not require a supermolecular calculation (as shown below). Importantly, the reconstruction-based embedding potentials can be used as benchmarks for future approximations of KEDF's, diagnosing issues in current approximations.

\subsubsection{Exact embedding using a reconstruction scheme}

We have now introduced all the necessary ingredients to establish a subsystem DFT scheme that eschews the use of a KEDF. Combining Eqs. 6.6, 6.13, and 6.15, we can obtain the exact embedding potential for a conventional subsystem DFT calculation as

$$
\begin{aligned}
v_{\text {emb }, 1}\left[\rho_{1}, \rho_{2}\right](\mathbf{r}) & =v_{\text {nuc } 2}(\mathbf{r})+\int \frac{\rho_{2}\left(\mathbf{r}^{\prime}\right)}{\left|\mathbf{r}-\mathbf{r}^{\prime}\right|} \mathrm{d} \mathbf{r} \\
& +v_{\text {xc }}^{\text {nad }}\left[\rho_{1}, \rho_{2}\right](\mathbf{r})+v_{t}^{\text {nad,exact }}\left[\rho_{1}, \rho_{2}\right](\mathbf{r}),
\end{aligned}
$$

where $\rho_{2}$ is fixed and $\rho_{1}$ is an initial density that will be optimized. The exact nonadditive kinetic potential has the expression:

$$
\begin{aligned}
v_{t}^{\text {nad,exact }}\left[\rho_{1}, \rho_{2}\right] & =\left.\frac{\delta T_{s}[\rho]}{\delta \rho}\right|_{\rho=\rho_{1}+\rho_{2}}-\left.\frac{\delta T_{s}[\rho]}{\delta \rho}\right|_{\rho=\rho_{1}} \\
& =-\tilde{v}_{s}\left[\rho_{1}+\rho_{2}\right]+\tilde{v}_{s}\left[\rho_{1}\right]+\Delta \mu,
\end{aligned}
$$

where the reconstructed potentials replace the functional derivative of the KEDF which is the main approximation of conventional DFT embedding.

In this scheme, before relaxing $\rho_{1}$, we need to first reconstruct the potentials for $\rho_{1}+\rho_{2}$ and $\rho_{1}$ to yield an exact nonadditive kinetic potential to use in the expression of the embedding potential. After relaxing $\rho_{1}$ in the embedding potential containing these terms, we can invert the roles of $\rho_{1}$ and $\rho_{2}$ and perform a full freeze\&thaw $(\mathrm{F} \& \mathrm{~T})$ calculation until self consistency, as sketched in the following subsection.

\subsubsection{The freeze\&thaw procedure}

We outline here the procedure we have optimized to conduct a freeze\&thaw run with the exact kinetic potentials is:

0) Perform isolated runs for the subsystems to obtain $\rho_{1}^{0}$ and $\rho_{2}^{0}$. Define $\rho_{\text {tot }}^{0}=$ $\rho_{1}^{0}+\rho_{2}^{0}$.

1a) Reconstruct $\tilde{v}_{s}\left[\rho_{\text {tot }}^{0}\right], \tilde{v}_{s}\left[\rho_{1}^{0}\right]$, and $\tilde{v}_{s}\left[\rho_{2}^{0}\right]$. 
1b) Construct the embedding potentials (Eq. 6.16) for subsystems 1 and 2 from the densities from point 0 and the $t_{s}^{\operatorname{nad}}\left[\rho_{1}^{0}, \rho_{2}^{0}\right]$ and $t_{s}^{\operatorname{nad}}\left[\rho_{2}^{0}, \rho_{1}^{0}\right]$ contributions obtained from the reconstructed potentials of point 1a (Eq. 6.17).

1c) Compute $\rho_{1}^{1}$ and $\rho_{2}^{1}$ using the embedding potentials from $1 \mathrm{~b}$, defining $\rho_{\text {tot }}^{1}=$ $\rho_{1}^{1}+\rho_{2}^{1}$.

etc.) Repeat points a-c until self-consistency.

The proposed scheme is preferable to defining and reconstructing the intermediate total density after updating subsystem 1 , namely, $\rho_{\text {tot }}^{i *}=\rho_{1}^{i}+\rho_{2}^{i-1}$, since this step would require two supermolecular reconstructions per freeze\&thaw cycle and these reconstructions are the bottleneck of this procedure. We also note that, in contrast to a conventional subsystem DFT calculation, in which the embedding potential $v_{\text {emb }}\left[\rho_{1}, \rho_{2}\right]$ can be (and usually is) updated during the minimization of $\rho_{1}$, in our scheme, such a "real-time" update of the embedding potential is impractical since the reconstruction steps are rather expensive computationally. However, at self-consistency, this lack of continuous updates is irrelevant. In practice, neither of these considerations are found to be an issue in the following and our F\&T cycles rapidly converge, as we will show below.

As initial guess potential, we use the following expression:

$$
v_{s}^{\text {guess }}[\rho](\mathbf{r})=v_{\text {Coul }}(\mathbf{r})-\frac{\zeta}{N} \int \frac{\rho(\mathbf{r})}{\left|\mathbf{r}-\mathbf{r}^{\prime}\right|} \mathrm{d} \mathbf{r},
$$

where, for the first cycle and subsystem 1 ,

$$
v_{\text {Coul }}^{(1)}(\mathbf{r})=v_{\text {nuc1 }}(\mathbf{r})+\int \frac{\rho_{1}^{(0)}\left(\mathbf{r}^{\prime}\right)}{\left|\mathbf{r}-\mathbf{r}^{\prime}\right|} \mathrm{d} \mathbf{r}^{\prime},
$$

while for all other cycles $i>1$ we need to include the influence of subsystem 2 as well:

$$
\begin{aligned}
v_{\text {Coul }}^{(i)}(\mathbf{r}) & =v_{\text {nuc1 }}(\mathbf{r})+\int \frac{\rho_{1}^{(i-1)}\left(\mathbf{r}^{\prime}\right)}{\left|\mathbf{r}-\mathbf{r}^{\prime}\right|} \mathrm{d} \mathbf{r}^{\prime}+ \\
& +v_{\text {nuc2 }}(\mathbf{r})+\int \frac{\rho_{2}^{(i-1)}\left(\mathbf{r}^{\prime}\right)}{\left|\mathbf{r}-\mathbf{r}^{\prime}\right|} \mathrm{d} \mathbf{r}^{\prime}
\end{aligned}
$$

Equivalent equations hold for subsystem 2 .

\subsection{Computational details}

We use the ADF program, version 2014 [9], in the PyADF scripting framework [10]. We employ the DZP [11] Slater basis set for the orbital basis and the DZP [11] charge-fitting basis as potential basis. We employ the PyADF interface [10] available with the ADF program for our calculations. We denote the frozen-density embedding calculations with orbital and potential basis sets localized on the subsystems as 
$\operatorname{FDE}(m)$ ("monomolecular basis set") and the results with a supermolecular basis set (both for the expression of the orbitals and of the potentials) as $\operatorname{FDE}(s)$. We allow a maximum density error (as defined in Eq. 6.7) of $e_{\text {dens }}<5 \cdot 10^{-3}$ a. u. for the reconstruction procedure of the supermolecules and $e_{\text {dens }}<2 \cdot 10^{-3}$ a. u. for those of the individual subsystems for all three example systems. For comparison with conventional DFT embedding, we run subsystem calculations using the PW91k [12] kinetic energy density functional. As exchange-correlation functional, we use BLYP $[13,14]$ in all calculations.

We use a modified version of the Molcas 7.4 [15] code for the CASPT2-in-DFT embedding calculations, where the embedding interface is adapted from the MolcasEmbed interface developed by Carter and co-workers [16, 17]. These calculations employ the aug-cc-pVDZ [18] basis set. We use the minimal CAS expansion for the molecules treated in WF/DFT, that is, CAS $(4,4)$ for MCP and CAS $(6,5)$ for acrolein.

We performed geometry minimization in the Gaussian09 code [19], using the cc-pVDZ [18] basis set and the BLYP [13,14] functional.

\subsection{Results}

To demonstrate our approach, we use the three test systems shown in Figure 6.1: Ammonia bonded to a water molecule, $s$-trans-acrolein (henceforth, acrolein) bonded to a water molecule, and methylenecyclopropene (MCP) bonded to three water molecules. For the excitation-energy calculations, we study the dark $\pi \rightarrow \pi^{*}$ excitation of MCP and the dark $n \rightarrow \pi^{*}$ excitation of $s$-trans-acrolein. The $\mathrm{MCP}+3 \times \mathrm{H}_{2} \mathrm{O}$ system is identical to the one discussed already as a test case in Chapter 4.

To assess the accuracy of the embedding potential, we perform two main tests: We compare the densities obtained with the converged F\&T procedure to supermolecular reference densities, and the excitation energies obtained by wave function calculations in the presence of the embedding potential from the last F\&T cycle to supermolecular excitation energies.

\subsubsection{Density errors for subsystem DFT}

The primary goal of any subsystem DFT calculation is to reproduce a supermolecular density. To test how well our F\&T method meets this standard, we compute the F\&T total densities after each F\&T cycle and compare them to densities computed via supermolecular KS calculations. In Table 6.1, we list the density errors yielded by monomolecular as well as supermolecular basis sets.

For all three molecules, our F\&T method in combination with the pure WY procedure yields converged densities with errors which are significantly smaller that those obtained in a standard subsystem DFT calculation with the approximate PW91k kinetic functional. If the monomolecular basis sets, $\operatorname{FDE}(m)$, are used as orbital and potential basis, the converged error on the sum of the two subsystem densities is however sizable for all three molecules. To show that the exact reconstruction scheme can reproduce the supermolecular results indeed exactly, we also perform the same 


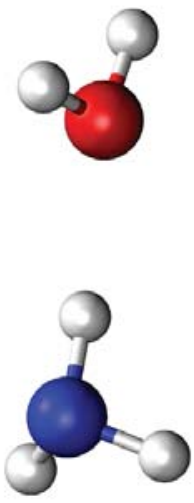

a)

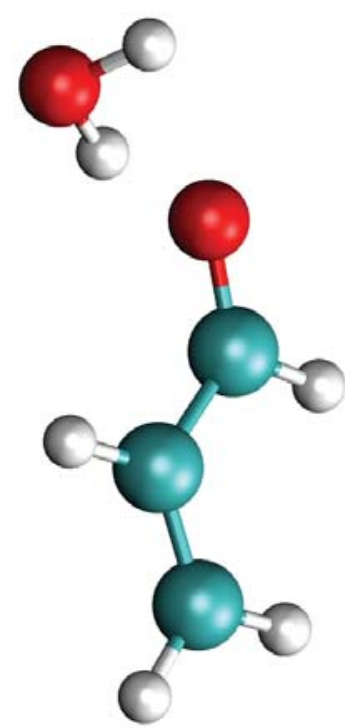

b)

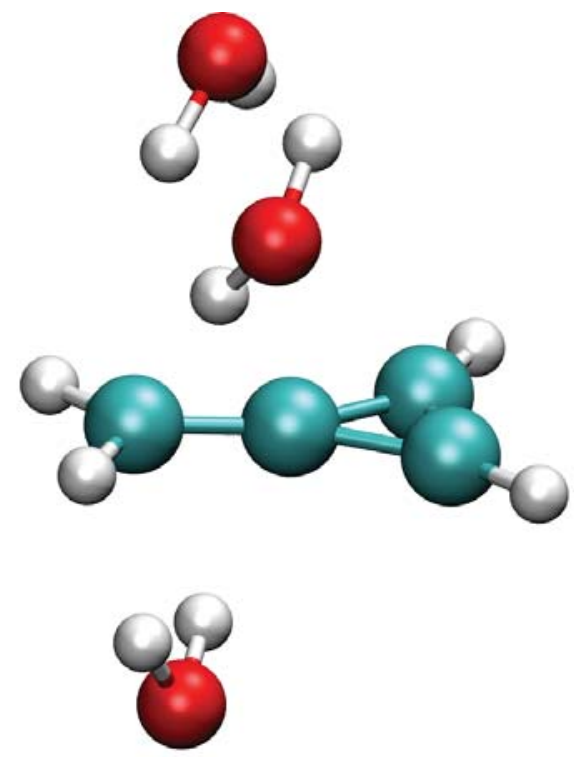

c)

Figure 6.1: The systems used in this study: a) Ammonia and a water molecule, b) acrolein and a water molecule, and c) MCP and three water molecules.

calculations using supermolecular orbital and potential basis sets, $\operatorname{FDE}(s)$, and find that the density error reduces by more than a factor of 6 and is comparable to the tolerance level of the WY procedure itself (in our case, chosen to be 0.005 a.u.) with the largest errors in the case of $\mathrm{MCP}+3$ waters still being only 0.013 a.u.

The density errors yielded by the virtual projection are however the largest, being up to 50\% larger than those obtained with PW91k. In Figures 6.2 and 6.3, we illustrate the evolution of the density error of acrolein as a function of number of F\&T cycles performed within $\operatorname{FDE}(m)$ and $\operatorname{FDE}(s)$, respectively. The other two systems display a very similar behavior. The fact that the density error produced by the virtual projection is significant is an issue that can however be averted by the use of larger orbital or potential basis sets and will be the subject of further study.

\subsubsection{Analysis of the embedding potentials}

In order to better understand the performance of virtual projection in our procedure, we plot the embedding potential acting on acrolein yielded by our exact freeze\&thaw computation of acrolein in water in Figure 6.4. We consider a configuration where the water and acrolein oxygens are at a distance of $2.7 \AA$. In the limit of large separation between the subsystems, the exact embedding potential in the region of the frozen subsystem (here, the water) is known to be zero (for instance, the nonadditive kinetic potential cancels the nuclear potential [20] in the environment). While in this case the separation is finite, the density overlap between the subsystems in the region of the water molecule is negligible and the large variations in the potential close to the oxygen nucleus of the water molecule are therefore unreasonable. Clearly, the 


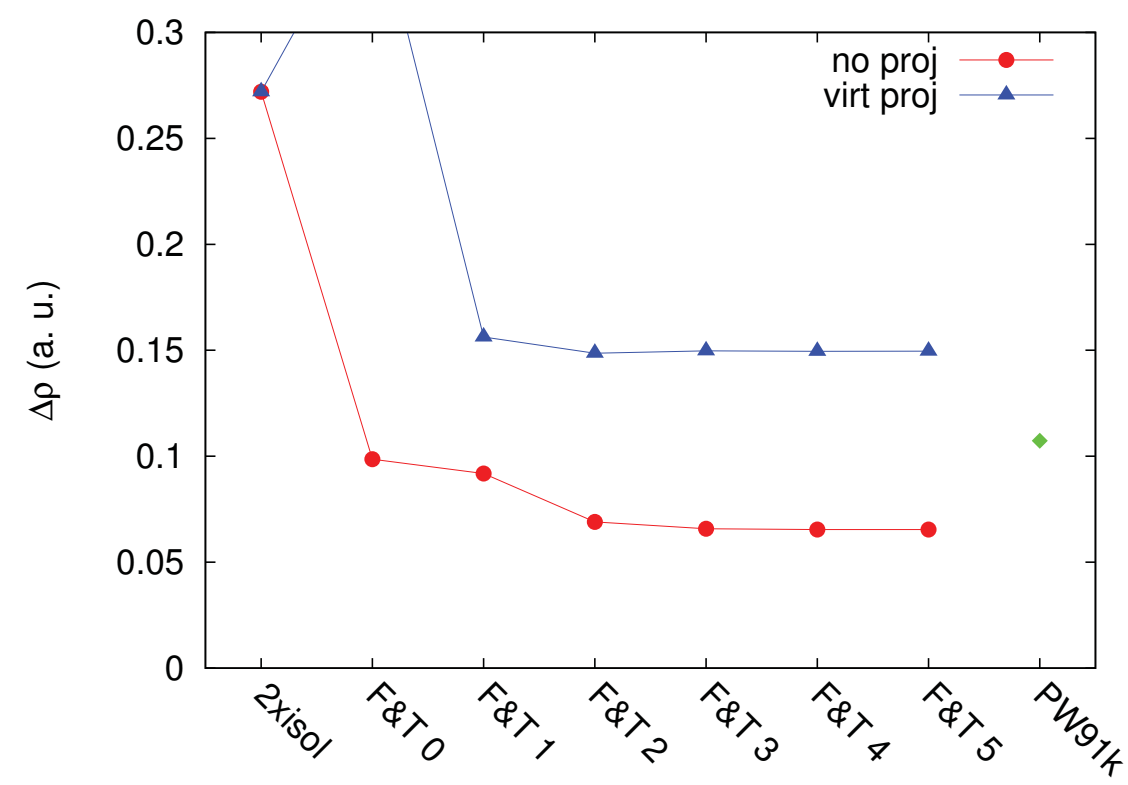

Figure 6.2: Acrolein: Density error of the sum of the two subsystem densities in F\&T cycles using a monomolecular basis set (as orbital and potential basis). The red circles represent F\&T calculations using the WY procedure (no proj) while the blue triangles represent $\mathrm{F} \& \mathrm{~T}$ calculations where we also apply the virtual projection (virt proj) during the construction of the potential. The green symbol represents the final value of a F\&T calculation with PW91k.

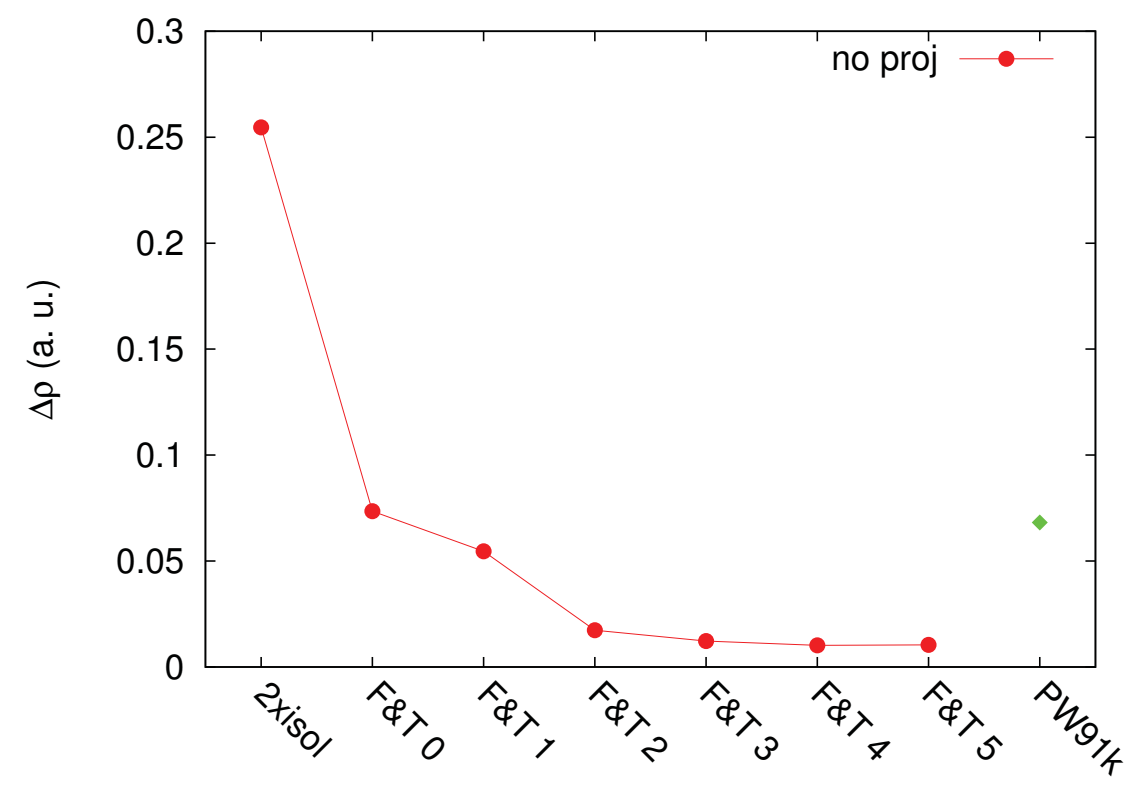

Figure 6.3: Acrolein: Density error of the sum of the two subsystem densities in F\&T cycles using a supermolecular basis set (as orbital and potential basis). The red circles represent F\&T calculations using the WY procedure (no proj) during the construction of the potential and the green symbol represents the final value of a F\&T calculation with PW91k. 


\begin{tabular}{ccccccc} 
& \multicolumn{3}{c}{$\operatorname{FDE}(m)$} & & \multicolumn{2}{c}{$\mathrm{FDE}(s)$} \\
\cline { 2 - 4 } \cline { 6 - 7 } System & WY & WY+virt & PW91k & & WY & PW91k \\
\hline ammonia+wat & 0.020 & 0.043 & 0.039 & & 0.003 & 0.031 \\
acrolein+wat & 0.065 & 0.150 & 0.107 & & 0.010 & 0.068 \\
MCP+3 wat & 0.090 & 0.245 & 0.161 & & 0.013 & 0.108 \\
\hline
\end{tabular}

Table 6.1: Density errors of the final sum of the two subsystem densities yielded by the F\&T procedure for ammonia, acrolein, and MCP in water, computed with FDE calculations with a monomolecular $[\operatorname{FDE}(m)]$ or supermolecular $[\operatorname{FDE}(s)]$ basis set for both the orbitals and the reconstructed potential. WY refers to the F\&T cycles employing only the WY scheme, while WY+virt denotes the runs applying the virtual projection after the WY scheme in the reconstruction.

virtual projection eliminates most of the sudden oscillations created by the Wu-Yang procedure and also evens out most of the strong repulsion close to the core of the environmental nuclei and is closer to fulfilling the criterion of a smooth potential in the environment than PW91k. The virtual projection also largely eliminates the dependence of the embedding potential on the density threshold $e_{\text {dens }}$ (data not shown here).

\subsubsection{WF/DFT results}

For MCP and acrolein, we compute excitation energies on the final embedding potentials constructed in the previous subsection using monomolecular basis sets. We refrain from using the embedding potentials determined using supermolecular basis sets for consistency with the wave function calculations which employ monomolecular basis sets, as practical calculations will need to do so. In Table 6.2, we report the excitation energies computed. In the interpretation of the data below, we must however keep in mind that our scheme does not include environmental response to the excitation (we are performing ground-state embedding) and agreement with the supermolecular value might be a result of cancellation of errors.

In the case of acrolein, the water molecule causes a blue shift of about $0.23 \mathrm{eV}$ on the excitation energy. The converged excitation energy computed using the exact F\&T procedure and the WY procedure is red-shifted compared to the reference value, which means that including environmental response to the excitation would worsen the agreement with the reference. Consequently, the red-shift observed in the excitation is most likely due to the unphysical features associated to the potentials obtained in the WY procedure. On the other hand, the excitation energies with virtually projected potentials are very close to PW91k and about $0.1 \mathrm{eV}$ blue shifted compared to the pure WY-based result.

In the case of MCP, the three water molecules cause a blue shift of about 0.57 $\mathrm{eV}$. The closest result to the supermolecular reference is the pure WY procedure, but it is again red-shifted compared to the reference value so the agreement will worsen if one allows a responsive environment. PW91k is about $0.1 \mathrm{eV}$ too high compared to the reference and the virtual projection causes a blue shift of more than $0.2 \mathrm{eV}$ 

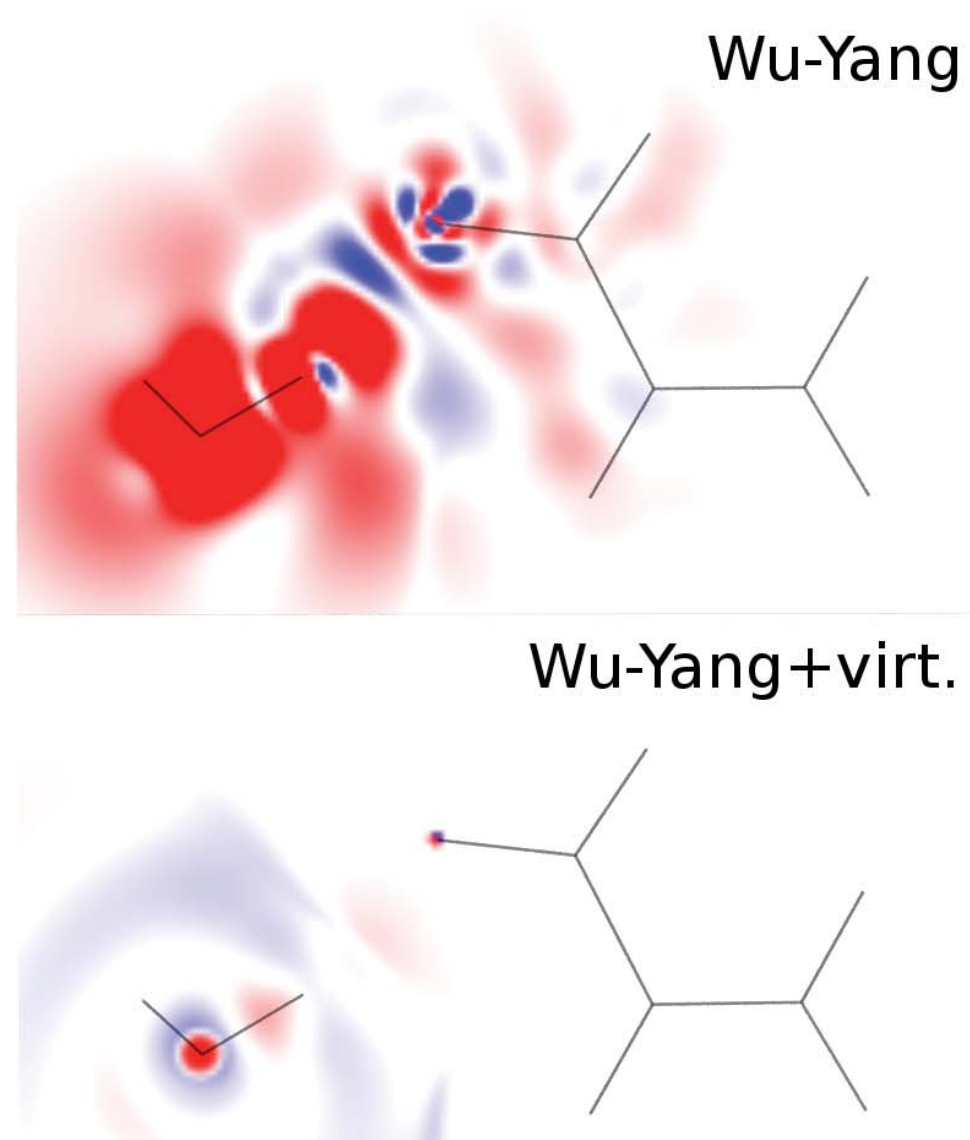

\section{PW91k}

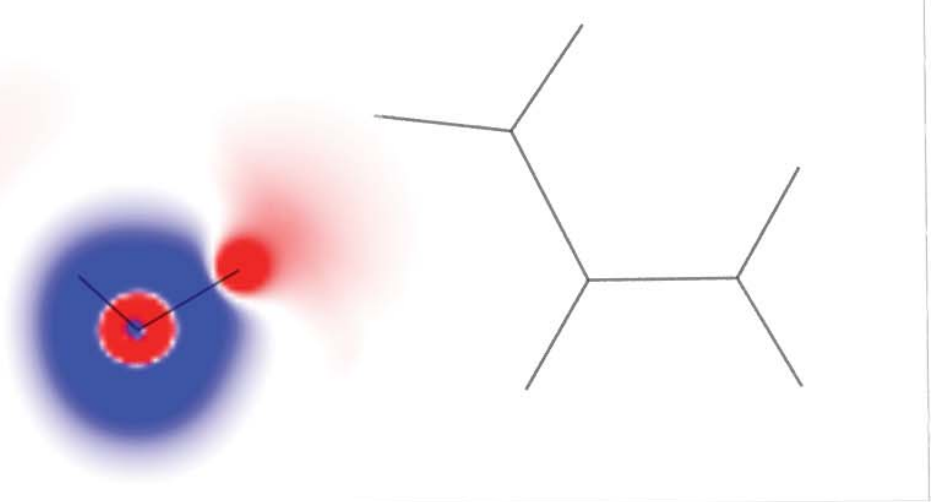

Figure 6.4: Acrolein: Embedding potential in the plane approximately defined by the $\mathrm{OH}$ bond in the water molecule and the oxygen atom in acrolein. Pure red refers to values higher than 0.5 hartree, pure blue refers to values lower than -0.5 hartree, white refers to zero, and shades of red and blue represent intermediate values. We illustrate the embedding potentials computed using $\mathrm{Wu}$-Yang reconstruction, the virtual projection (middle), and PW91k. The black lines refer to the bonds in the water (bottom left) and the acrolein molecules (right). 


\begin{tabular}{ccccccc} 
& \multicolumn{3}{c}{ CASPT2/DFT } & & \multicolumn{2}{c}{ CASPT2 } \\
\cline { 2 - 4 } \cline { 6 - 7 } System & WY & WY+virt & PW91k & & isolated & super \\
\hline acrolein+wat & 3.669 & 3.763 & 3.775 & & 3.477 & 3.705 \\
MCP+3 wat & 4.640 & 4.865 & 4.779 & & 4.111 & 4.684
\end{tabular}

Table 6.2: CASPT2/DFT excitation energies (eV) using the embedding potentials from the exact F\&T procedure $(\operatorname{FDE}(m))$ for acrolein and MCP. The CASPT2 excitation energies of the isolated molecules as well as the supermolecule are also reported.

compared to the results yielded by only the WY procedure. We stress again that a somewhat blue-shifted excitation energy is not unreasonable since we are computing the excitation energy in an embedding potential obtained in a ground-state procedure. Based on the available data, we can only conclude that improving the ground-state potential in a wave function in DFT calculation does not necessarily improve the agreement of the excitation energy with the reference since we are still neglecting the relaxation of the environment in response to the excitation of the embedded species.

\subsection{Conclusions}

We implemented and performed for the first time subsystem DFT calculations with an exact freeze\&thaw procedure based on reconstructed potentials, thereby without the use of an approximate kinetic-energy density functional. In the case of supermolecular basis sets, the procedure yields densities that are very close to the results of reference supermolecular KS calculations and far better than those yielded by approximate kinetic-energy functionals. Virtual projection eliminates unphysical oscillations in the potential at the cost of increased density errors. The excitation energies obtained in wave function calculations in the embedding potentials obtained with our exact freeze\&thaw procedure are reasonable but there are large spreads of up to 0.2 $\mathrm{eV}$ depending on the chosen reconstruction scheme. For the two systems considered here, the use of only the WY procedure to generate the potentials yields red-shifted excitation energies compared to the reference, while the results when employing the virtual projection are similar or higher than those obtained with PW91k, both of them being blue-shifted compared to the reference excitation energies. While embedding potentials determined using the pure WY scheme give apparently the excitation energies closest to the reference, we should keep in mind that this is likely the result of cancellation of errors due to the neglect of environmental response, which will in fact further red-shift the excitation energies. Further studies are necessary to understand how to reduce the currently quite large density errors produced by the virtual projection procedure, most likely through the expansion of the orbital or potential basis set. The reduction of this error will allow us to generate both robust embedding potentials and accurate total densities by combining our exact $\mathrm{F} \& \mathrm{~T}$ procedure with the virtual projection reconstruction approach. 


\section{Bibliography}

[1] R. G. Parr and W. Yang, Density-Functional Theory of Atoms and Molecules (Oxford University Press, 1989).

[2] W. Yang and Q. Wu, Phys. Rev. Lett. 89, 143002 (2002).

[3] Q. Wu and W. Yang, J. Chem. Phys. 118, 2498 (2003).

[4] S. Fux, C. R. Jacob, J. Neugebauer, L. Visscher, and M. Reiher, J. Chem. Phys. 132, 164101 (2010).

[5] C. R. Jacob, J. Chem. Phys. 135, 244102 (2011).

[6] E. Fermi and E. Amaldi, Accad. Ital. Rome 6, 117 (1934).

[7] T. Heaton-Burgess, F. A. Bulat, and W. Yang, Phys. Rev. Lett. 98, 256401 (2007).

[8] F. R. Manby, M. Stella, J. D. Goodpaster, and T. F. Miller, J. Chem. Theory Comput. 8, 2564 (2012), pMID: 22904692.

[9] ADF2014, SCM, Theoretical Chemistry, Vrije Universiteit, Amsterdam, The Netherlands, http://www.scm.com (access date: 06/06/2015).

[10] C. R. Jacob, S. M. Beyhan, R. E. Bulo, A. S. P. Gomes, A. W. Götz, K. Kiewisch, J. Sikkema, and L. Visscher, J. Comput. Chem. 32, 2328 (2011).

[11] E. V. Lenthe and E. J. Baerends, J. Comput. Chem. 24, 1142 (2003).

[12] A. Lembarki and H. Chermette, Phys. Rev. A 50, 5328 (1994).

[13] A. D. Becke, Phys. Rev. A 38, 3096 (1988).

[14] C. T. Lee, W. T. Yang, and R. G. Parr, Phys. Rev. B 37, 785 (1988).

[15] F. Aquilante, L. D. Vico, N. Ferré, G. Ghigo, P.-Å. Malmqvist, P. Neogrády, T. B. Pedersen, M. Pitonák, M. Reiher, B. O. Roos, L. Serrano-Andrés, M. Urban, V. Veryazov, and R. Lindh, J. Comp. Chem. 31, 224 (2010).

[16] P. Huang and E. A. Carter, J. Chem. Phys. 125, 084102 (2006).

[17] S. Sharifzadeh, P. Huang, and E. A. Carter, Chem. Phys. Lett. 470, 347 (2009). 
[18] T. H. Dunning Jr, J. Chem. Phys. 90, 1007 (1989).

[19] M. J. Frisch, G. W. Trucks, H. B. Schlegel, G. E. Scuseria, M. A. Robb, J. R. Cheeseman, G. Scalmani, V. Barone, B. Mennucci, G. A. Petersson, H. Nakatsuji, M. Caricato, X. Li, H. P. Hratchian, A. F. Izmaylov, J. Bloino, G. Zheng, J. L. Sonnenberg, M. Hada, M. Ehara, K. Toyota, R. Fukuda, J. Hasegawa, M. Ishida, T. Nakajima, Y. Honda, O. Kitao, H. Nakai, T. Vreven, J. J. A. Montgomery, J. E. Peralta, F. Ogliaro, M. Bearpark, J. J. Heyd, E. Brothers, K. N. Kudin, V. N. Staroverov, R. Kobayashi, J. Normand, K. Raghavachari, A. Rendell, J. C. Burant, S. S. Iyengar, J. Tomasi, M. Cossi, N. Rega, J. M. Millam, M. Klene, J. E. Knox, J. B. Cross, V. Bakken, C. Adamo, J. Jaramillo, R. Gomperts, R. E. Stratmann, O. Yazyev, A. J. Austin, R. Cammi, C. Pomelli, J. W. Ochterski, R. L. Martin, K. Morokuma, V. G. Zakrzewski, G. A. Voth, P. Salvador, J. J. Dannenberg, S. Dapprich, A. D. Daniels, Ö. Farkas, J. B. Foresman, J. V. Ortiz, J. Cioslowski, and D. J. Fox, Gaussian 09 Revision A.2, gaussian 09 Revision A.2, Gaussian Inc. Wallingford CT 2009.

[20] C. R. Jacob, S. M. Beyhan, and L. Visscher, J. Chem. Phys. 126, 234116 (2007). 


\section{Chapter 7}

\section{Full Configuration Interaction excitations of ethene and butadiene: Resolution of an ancient question ${ }^{\dagger}$}

We employ the recently developed full configuration interaction quantum Monte Carlo (FCIQMC) method to compute the $\pi \rightarrow \pi^{*}$ vertical excitation energies of ethene and all-trans butadiene. These excitations have been the subject of extensive theoretical studies, and their location with respect to the corresponding absorption band maximum the source of a long lingering debate. Here, we reliably estimate the vertical excitations of ethene and butadiene by performing FCIQMC calculations for spaces as large as $10^{18}$ and $10^{29}$ Slater determinants, respectively. For ethene, we obtain a vertical excitation energy in the range of 7.89-7.96 eV, depending on the particular equilibrium ground-state geometry employed, and definitely higher than the absorption maximum located at $7.66 \mathrm{eV}$. For the computationally more challenging case of butadiene, our calculations provide a robust estimate of about $6.3 \mathrm{eV}$ for this excitation, that is, $0.4 \mathrm{eV}$ higher than the corresponding absorption band maximum. Our FCIQMC excitation energies represent a reliable benchmarking reference for future calculations.

\subsection{Introduction}

The $\pi \rightarrow \pi^{*}$ vertical excitation energies of ethene and all-trans butadiene have been the subject of a remarkably large number of theoretical investigations [1-24]. A wide variety of ab initio methods has been employed but little consensus has been reached on the exact location of the vertical excitations to the $1^{1} B_{1 u}$ and $1^{1} B_{u}$ states of ethene and butadiene, respectively. Importantly, despite the evident difficulty of

$\dagger$ This chapter has been published as C. Daday, S. Smart, G. H. Booth, A. Alavi, and C. Filippi, "Full Configuration Interaction excitations of ethene and butadiene: Resolution of an ancient question", J. Chem. Theory Comput. 2014, 8, 4441-4451 
obtaining a reliable estimate of their excitation energies, ethene and butadiene are often chosen as test cases to assess the accuracy of theoretical approaches for excited states $[3,5,11,12,14,24]$.

Experimentally, the absorption spectrum of ethene in the far ultraviolet region consists of a broad continuous band with a maximum at $7.66 \mathrm{eV}[25,26]$. A direct comparison between the experimental absorption maximum and the computed vertical excitation energy is meaningful if the transition probability is largest at the ground-state minimum. Since the Franck-Condon envelope might be significantly distorted for the active $1^{1} B_{1 u}$ state of ethene, an excitation energy of $7.8 \mathrm{eV}$ was estimated based on an intensity weighted average energy calculated from the experimental spectrum [6]. Moreover, an earlier theoretical study indicated that nonadiabatic effects should also be taken in account, which will lead to a further blue shift in the location of the vertical excitation with respect to the band maximum [1]. Several attempts to directly compute the full spectrum have unfortunately not clarified the issue since qualitative agreement in the description of the intensity distribution has been obtained with rather different theoretical models, for instance, either discarding or including nonadiabatic couplings to other electronic excited states [1,9,10,27].

Most calculations of the $1^{1} B_{1 u}$ vertical excitation of ethene obtain values higher than the experimental absorption maximum. The most reliable complete-activespace second-order perturbation theory (CASPT2) estimates lie between 7.8 and 8.0 $\mathrm{eV}[5,12,15]$, and coupled cluster (CC) approaches up to perturbative third order place the excitation in the same energy range $[4,9,12]$. In contrast, two investigations [7,13] obtain a vertical excitation energy of the order of $7.7 \mathrm{eV}$, that is, very close to the experimental absorption maximum at $7.66 \mathrm{eV}$ and therefore exclude a significant impact of nonadiabatic vibronic effects on the location of the absorption maximum. These calculations employ multi-reference coupled-cluster and configuration-interaction approaches on appropriately constructed molecular orbitals [7] or multi-reference perturbation approaches on orbitals obtained with restricted active space methods [13]. The difficulty in computing the $\pi \rightarrow \pi^{*}$ vertical excitation energy of ethene is evident from the spread of theoretical estimates, and is generally attributed to the so-called valence-Rydberg mixing, namely, the presence of a Rydberg-like state close in energy to the excited state of interest, which is instead essentially valence in nature. Unless electron correlation is accurately described, the Rydberg state might undesirably mix in, rendering the resulting wave function unduly diffuse and its energy unreliable. A recent study comparing the many different computed values in the literature [12] concludes that "the vertical excitation energy of the $1^{1} B_{1 u}$ state can still not be assigned precisely, but should lie between 7.7 and $8.0 \mathrm{eV}$ ".

For butadiene, the situation is equally confused. The $1^{1} B_{u}$ state has been experimentally investigated by electron impact and optical spectroscopy and the absorption spectrum is characterized by broad bands between 5.7 and $6.3 \mathrm{eV}$ with the intensity maximum at $5.92 \mathrm{eV}$ [28-30]. Similarly to the case of ethene, the vertical excitation energy is believed to be blue-shifted with respect to this value. Theoretically, valence-Rydberg mixing represents a problem also for the description of the valence $1^{1} B_{u}$ state of butadiene and a rather large spread of computational estimates can in 
fact be found in the literature. Available CASPT2 calculations give excitation energies in the range of 6.1-6.3 eV $[3,14,18]$ but these single-state studies are hard to compare as they employ different zero-order Hamiltonians $[14,18]$ or, in the presence of diffuse functions, lead to a too diffuse state due to the lack of a multi-state treatment of the close Rydberg state [3]. Various flavors of CC calculations yield values between 6.2 and $6.4 \mathrm{eV}[4,17,22,23]$. A recent multi-reference coupled cluster study obtains an excitation energy of $6.18 \mathrm{eV}$ [20] with the use of molecular orbitals constructed with a similar procedure to the one adopted by the same group for ethene [7].

Here, we employ the recently developed full configuration interaction quantum Monte Carlo (FCIQMC) method [31-35] to compute the bright $\pi \rightarrow \pi^{*}$ excitations of ethene and all-trans butadiene. While a conventional full configuration interaction (FCI) calculation would not be feasible for these systems, the FCIQMC approach offers a computationally costly but practical route to obtain the FCI limit of these excitation energies with several orders of magnitude less memory requirements. We recall that the largest reported FCI calculation has been performed for the nitrogen molecule and a space of $10^{10}$ Slater determinants [36]. In contrast, we treat here FCI spaces of $10^{18}$ determinants for ethene and $10^{29}$ determinants for butadiene corresponding to a partially augmented triple-valence basis and a partial triple-valence basis, respectively.

The article is organized as follows. In Section 7.2, we give a short description of the FCIQMC method and summarize the aspects specific to the current calculations. In Section 7.3, we present our choice of basis sets and geometries. In Section 7.4, we discuss our results and compare them with previous calculations in the literature. Section 7.5 summarizes our conclusions.

\subsection{The FCIQMC method}

The recently developed FCIQMC approach yields the FCI solution of the Schrödinger equation stochastically and with significantly less memory requirements than conventional FCI [31-35]. The method is based on formulating projection Monte Carlo in Slater determinant space, so the ground-state wave function is obtained as

$$
\Psi_{0}=\lim _{\tau \rightarrow \infty} e^{-\tau(\mathcal{H}-E)} \Psi^{(0)},
$$

where the trial energy $E$ is adjusted to the value of the ground-state energy and $\Psi^{(0)}$ is some initial wave function. If we express the wave function in the basis of Slater determinants, $D_{i}$, generated by arranging the electrons in a given set of spin orbitals, we obtain the FCI representation of the wave function,

$$
\Psi=\sum_{i} c_{i} D_{i}
$$

The projection (Eq. 7.1) formulated in this determinantal basis is then equivalent to a set of coupled equations for the evolution of the determinant coefficients in the CI 
expansion as

$$
-\frac{d c_{i}}{d \tau}=\left(H_{i i}-E\right) c_{i}+\sum_{j \neq i} H_{i j} c_{j},
$$

where $H_{i j}$ are the matrix elements of the Hamiltonian between the Slater determinants.

The evolution of the CI coefficients is simulated through the dynamics of a population of $N_{w}$ walkers which are distributed over the determinants and can carry a positive or a negative sign. The parameter $\tau$ is discretized and the walkers evolve according to the terms in the above equation (Eq. 7.3): Walkers can spawn new walkers on to other determinants (off-diagonal terms) and each walker can die or clone (diagonal term) depending on the trial energy, which is varied to keep the population roughly constant at a target value. After every step, walkers of opposite sign on the same determinant annihilate each other to ensure sign coherence [31,37]. While the algorithm still scales exponentially with the system size, FCIQMC achieves convergence to the FCI limit with populations of walkers that are a fraction of the dimensions of the determinant space $\left(N_{\mathrm{FCI}}\right)$ as demonstrated for systems with spaces ranging from $10^{9}$ to $10^{14}$ determinants [31]. The gain with respect to a conventional FCI simulation is system dependent and rather hard to predict, being for instance minor in the relatively simple case of methane and significant for other notoriously difficult molecules.

A dramatic improvement to this original formulation is represented by the socalled initiator approach [32], which significantly reduces the population needed and extends the applicability of FCIQMC to more complex systems and larger basis sets [32-35]. In the initiator approach, spawning to empty determinants is only allowed from determinants, dubbed "initiators", with a number of walkers exceeding a certain threshold, $n_{\text {add }}$. Except for the special case of "double-spawning", the progeny of non-initiator determinants survive only if they are spawned onto an already occupied determinant. Double-spawning occurs if two non-initiators simultaneously spawn walkers onto the same (originally empty) determinant: If the two progeny are of the same sign, they are allowed to survive. Such double-spawning events are rare, but are found to be helpful in establishing sign coherence [32]. Clearly, the initiator algorithm reduces to the FCIQMC algorithm in the limit of a large number of walkers, since in this limit, all determinants acquire an occupancy and therefore walkers spawned from non-initiators will survive as per the original FCIQMC algorithm. In practice, the convergence of the correlation energy with walker number to the FCI result is very rapid and occurs well before all determinants have acquired an occupation. It does however have a system dependence: Generally speaking, weakly correlated systems will converge more rapidly with walker number than strongly correlated ones. The standard protocol of an initiator-FCIQMC simulation is to perform calculations with increasing numbers of walkers, and to monitor the convergence of the energy. It is found that the initiator-FCIQMC method is still characterized by exponential scaling but the exponent is now significantly smaller than with FCIQMC. In other words, the relation between the size of the walker pop- 
ulation and dimension of the FCI space is given by

$$
N_{w} \propto\left(N_{\mathrm{FCI}}\right)^{\alpha},
$$

but the initiator approach significantly reduces the value of $\alpha$ with respect to the original FCIQMC algorithm, characterized by walker populations roughly linear with the FCI size, $\alpha \approx 1$ [31]. For calculations with the initiator approach, values of $\alpha$ between 0.16 for neutral and ionized atoms up to sodium [34] and 0.34 for $\mathrm{C}_{2}$ [35] have been reported.

Here, we employ the initiator variant of the FCIQMC method and report below the value of $n_{\text {add }}$ used in each calculation. We construct the Slater determinants from the Hartree-Fock orbitals and expand the wave functions on spin-flip combinations of determinants constructed to exclude odd-spin states [35]. This is particularly relevant for the computation of the $1^{1} B_{1 u}$ and $1^{1} B_{u}$ excited states of ethene and butadiene, respectively, because it ensures that the solution converges to the singlet state of the chosen spatial symmetry and not to the triplet state, which is lower in energy. While even-spin states other than the singlet are not excluded, they are significantly higher in energy and therefore projected out in the dynamics. All simulations are started from one walker on the reference configuration state function, which is the HartreeFock determinant in the ground-state calculations and a spin-flip combination for the excited states. We grow the walker population to a target value while keeping the trial energy fixed to the Hartree-Fock value, and then release the trial energy into variable mode to maintain the population stationary. The energy is computed with the so-called mixed estimator as

$$
E_{\text {proj }}=\frac{\left\langle D_{0}|\mathcal{H}| \Psi_{\mathrm{FCI}}\right\rangle}{\left\langle D_{0} \mid \Psi_{\mathrm{FCI}}\right\rangle}=\sum_{j}\left\langle D_{0}|\mathcal{H}| D_{j}\right\rangle \frac{\left\langle N_{j}\right\rangle}{\left\langle N_{0}\right\rangle},
$$

where the coefficient $c_{i}$ in the expansion is estimated as the average of the sum, $N_{i}$, of the signs of all walkers on that determinant. In this energy expression, we still denote with $D_{i}$ the corresponding spin-flip combination of determinants.

\subsection{Computational details}

All FCIQMC calculations are performed with the Alavi group code (NECI) and the setup of the one- and two-electron integrals and molecular orbitals necessary for FCIQMC is carried out with the Q-Chem code [38]. We employ the frozen-core approximation and keep the molecular orbitals corresponding to the 1s electrons of the carbon atoms doubly occupied. This amounts to freezing 4 and 8 spin-orbitals for ethene and butadiene, respectively.

The ground-state equilibrium structures are optimized at the MP2 level with the Gaussian 09 code [39] and at the CASPT2 level with the MOLCAS code version 7.4 [40]. We investigate the basis-set dependence of the excitation energies within linear response coupled-cluster (CC) theory [41,42] at the singles and doubles (CCSD) [43] and approximate third (CC3) [44, 45] orders, using the Dalton 


\begin{tabular}{llcc}
\hline Basis & Label & Aug-C & Aug-H \\
\hline ANO-L-VDZP & & & \\
& $+\mathrm{C}(\mathrm{p})$ & $1 \mathrm{p}$ & \\
& $+\mathrm{C}$ & $1 \mathrm{~s} 1 \mathrm{p} 1 \mathrm{~d}$ & \\
& + all & $1 \mathrm{~s} 1 \mathrm{p} 1 \mathrm{~d}$ & $1 \mathrm{~s} 1 \mathrm{p}$ \\
& + double & $2 \mathrm{~s} 2 \mathrm{p} 2 \mathrm{~d}$ & $1 \mathrm{~s} 1 \mathrm{p}$ \\
& & & \\
ANO-L-VTZP & & & \\
ANO-L-VTZP & & & \\
& $+\mathrm{C}(\mathrm{s}, \mathrm{p}, \mathrm{d})$ & $1 \mathrm{~s} 1 \mathrm{p} 1 \mathrm{~d}$ & \\
& + all & $1 \mathrm{~s} 1 \mathrm{p} 1 \mathrm{~d} 1 \mathrm{f}$ & $1 \mathrm{~s} 1 \mathrm{p} 1 \mathrm{~d}$ \\
& + double & $2 \mathrm{~s} 2 \mathrm{p} 2 \mathrm{~d} 2 \mathrm{f}$ & $1 \mathrm{~s} 1 \mathrm{p} 1 \mathrm{~d}$ \\
& & & \\
ANO-L-VQZP & & & \\
& $+\mathrm{C}$ & $1 \mathrm{~s} 1 \mathrm{p} 1 \mathrm{~d} 1 \mathrm{f}$ & \\
& + all & $1 \mathrm{~s} 1 \mathrm{p} 1 \mathrm{~d} 1 \mathrm{f}$ & $1 \mathrm{~s} 1 \mathrm{p} 1 \mathrm{~d}$ \\
\hline
\end{tabular}

Table 7.1: Number and type of diffuse functions employed to augment the ANO-LVXZP basis sets on the carbon (Aug-C) and hydrogen (Aug-H) atoms. The label we assign to the resulting augmented basis set is also listed.

2.0 program [46]. We also perform equation-of-motion coupled cluster calculations at the singles, doubles and triples (EOM-CCSDT) order [47,48] using the CFOUR program, version 1.0 [49]. The frozen-core approximation is employed in the CC calculations.

\subsubsection{Choice of basis sets}

To optimize the ground-state equilibrium structures and investigate their basis set dependence, we employ the Dunning's correlation consistent cc-pVXZ basis sets [50]. For the computation of the excitation energies, we use the ANO-L-VXZP basis sets [51] with the following contraction scheme: ANO-L-VDZP [3s2p1d]/[2s1p], ANO-L-VTZP [4s3p2d1f]/[3s2p1d], ANO-L-VQZP [5s4p3d2f]/[4s3p2d] and ANOL-V5ZP [6s5p4d3f]/[5s4p3d].

In Table 7.1, we detail how we augment the ANO-L-VXZP basis sets with the diffuse functions from the corresponding aug-cc-pVXZ and d-aug-cc-pVXZP basis sets [52], and how we label the various augmented basis sets. For example, in the $\mathrm{VDZP}+\mathrm{C}(\mathrm{p})$ set, we only augment the basis on the $\mathrm{C}$ atoms with the diffuse $p$ function from the aug-cc-pVDZ basis while we use the full augmentation on the $\mathrm{C}$ atoms in the VDZP+C basis set. In the VDZP+double basis set, the augmentation from the $\mathrm{d}$-aug-cc-pVDZ is employed on the $\mathrm{C}$ atoms in combination with the augmentation from the aug-cc-pVDZ on the $\mathrm{H}$ atoms. Finally, we also construct a VTZP' basis set without augmentation, where the VTZP basis set without f-functions is employed on the carbon atoms, and the VDZP basis set on the hydrogen atoms. 


\subsubsection{Ground-state equilibrium geometries}

Previous calculations of the vertical excitation energies of ethene and all-trans butadiene have been performed on a variety of geometrical models either optimized with different ab initio methods or from different experimental studies. For butadiene, in particular, there are at least four different structures derived from independent experimental data [53-56], which give a spread of about $0.012 \AA$ in the carbon-carbon double bond, well outside the reported error bars, as shown in Table 7.2. For ethene, most previous calculations of the vertical excitation energy have employed the geometry from the experimental study of Ref. 57 but it is worth noting that another structure has been reported with a carbon-carbon bond length not compatible within the given error bar [58] (see Table 7.2). Given the uncertainty in the structures derived from experiments, we generate here the ground-state equilibrium structures with the MP2 method and validate our choice against CASPT2 geometries.

We optimize the MP2 geometries with cc-pVXZ basis set series and list the carbon-carbon bond lengths in Table 7.2. We impose $\mathrm{D}_{2 \mathrm{~h}}$ symmetry for ethene and $\mathrm{C}_{2 \mathrm{~h}}$ symmetry for butadiene, and place ethene in the yz-plane along the $\mathrm{z}$-axis, so the $\pi \rightarrow \pi^{*}$ excited state has $\mathrm{B}_{1 u}$ symmetry. We find that the cc-pVQZ basis set gives converged bond lengths to better than $0.001 \AA$ while smaller basis sets yield slightly elongated bond lengths with a discrepancy of about $0.015 \AA$ for the smallest cc-pVDZ basis set. To assess the quality of the MP2/cc-pVQZ geometries, we also optimize the structures with the CASPT2/cc-pVQZ method. We use a CAS $(2,2)$ and a CAS $(4,4)$ expansion for ethene and butadiene, respectively, where all $\pi$ electrons in the reference are correlated in an equal number of $\pi$ orbitals. For ethene, we also consider the larger CAS $(6,6)$ active space where $\sigma$ and $\pi$ electrons are correlated over the orbitals $\left(1 b_{2 g}, 1 b_{3 u}, 3 a_{g}, 1 b_{3 g}, 2 b_{2 u}, 3 b_{1 u}\right)$ as in Ref. 7. The differences between the CASPT2 and MP2 bond lengths optimized with the same cc-pVQZ basis are smaller than 0.004 and $0.002 \AA$ in ethene and butadiene, respectively. We also note that our MP2 geometries are in excellent agreement with the equilibrium structures recently obtained by combining rotational spectroscopic data with vibration-rotation constants from quantum chemical calculations [59] (denoted with "Semi-exp" in Table 7.2).

We also investigate the dependence of the vertical excitation energies on the choice of geometry at the CCSD level using the ANO-L-VQZP basis for ethene, and the ANO-L-VTZP+all basis set for butadiene. We find that the vertical excitation energies of both molecules are rather sensitive to relatively small changes in bond lengths. For instance, the use of MP2 geometries optimized with the cc-pVDZ basis yields excitation energies red-shifted by as much as $0.1 \mathrm{eV}$ with respect to the values obtained on the MP2/cc-pV5Z structures. The excitations computed on the cc-pVQZ structures are instead converged to better than $0.01 \mathrm{eV}$. We also compute the CCSD excitations on the four experimental geometries of butadiene, where we use the available experimental parameters but the carbon-hydrogen bond lengths and HCC bond angles from the CASPT2 equilibrium geometry. The spread of $0.012 \AA$ in the carbon-carbon double bond among the four geometries translates into variations of $0.1 \mathrm{eV}$ in the computed vertical excitation energy. Clearly, the sensitivity to the 


\begin{tabular}{llcccc}
\hline Molecule & Method & Basis & $\mathrm{C}=\mathrm{C}$ & $\mathrm{C}-\mathrm{C}$ & $\Delta \mathrm{E}(\mathrm{eV})$ \\
\hline Ethene & MP2 & $\mathrm{D}$ & 1.346 & & 7.95 \\
& MP2 & $\mathrm{T}$ & 1.332 & & 8.05 \\
& MP2 & $\mathrm{Q}$ & 1.330 & & 8.06 \\
MP2 & 5 & 1.329 & & 8.07 \\
& CASPT2/CAS(2,2) & $\mathrm{Q}$ & 1.332 & & 8.05 \\
& CASPT2/CAS(6,6) & $\mathrm{Q}$ & 1.333 & & 8.04 \\
Exp [57] & & 1.339 & & 8.00 \\
Exp [58] & & $1.334(1)$ & & 8.03 \\
Semi-exp [59] & & $1.3305(10)$ & & 8.06 \\
Butadiene & MP2 & $\mathrm{D}$ & 1.353 & 1.464 & 6.27 \\
& MP2 & $\mathrm{T}$ & 1.340 & 1.453 & 6.35 \\
MP2 & $\mathrm{Q}$ & 1.338 & 1.451 & 6.36 \\
MP2 & 5 & 1.338 & 1.450 & 6.36 \\
CASPT2/CAS(4,4) & $\mathrm{Q}$ & 1.340 & 1.452 & 6.35 \\
Exp [53] & & $1.343(9)$ & $1.467(2)$ & 6.36 \\
Exp [54] & & $1.341(2)$ & $1.463(3)$ & 6.36 \\
Exp [55] & & $1.349(1)$ & $1.467(2)$ & 6.31 \\
Exp [56] & & 1.337 & 1.467 & 6.40 \\
Semi-exp [59] & & $1.3376(10)$ & $1.4539(10)$ & 6.37 \\
\hline
\end{tabular}

Table 7.2: Carbon-carbon bond lengths ( $⿱$ ) of ethene and butadiene optimized with the MP2 and CASPT2 methods and the cc-pVXZ basis set with X=D, T, Q, 5. We also list the CCSD vertical excitation energies $(\mathrm{eV})$ computed with the ANO-LVQZP basis set for ethene, and the ANO-L-VTZP+all basis set for butadiene.

choice of geometry should be taken into account when comparing theoretical studies employing different structural models of ethene and butadiene.

Having validated our choice of equilibrium geometries, we perform the FCIQMC calculations on the MP2/cc-pVQZ structures. This combination of method and basis has also the advantage of being easily applicable to consistently extend the present FCIQMC study to a larger set of molecules. For ethene, we also perform FCIQMC calculations on the experimental geometry from Ref. 57 since this geometry has been so often employed in previous theoretical studies of the same vertical excitation.

\subsection{Results}

We present here the FCIQMC vertical excitation energies of ethene and butadiene computed with various basis sets. In all calculations, we use the initiator-FCIQMC approach with thresholds of $n_{\text {add }}=3$ for ethene and $n_{\text {add }}=5$ for butadiene. These values minimize the required size of walker populations for all basis sets in the two systems. For each basis set, the number of walkers is increased until no significant change is observed in the correlation energy. We converge the ground and excited states separately and, in general, the excited state requires more walkers for conver- 
gence. Nevertheless, we report the energies obtained with the same population size for both states to further reduce the statistical error on the excitation energy. To estimate the remaining basis set error, we also perform $\mathrm{CC}$ calculations with the same basis sets as in the FCIQMC runs as well as with larger basis sets. In all the computations below, we employ the augmented ANO-L basis sets described in Table 7.1, and omit at times ANO-L in the acronym.

\subsubsection{Ethene}

In Table 7.3, we report the FCIQMC total energies of the $1^{1} \mathrm{~A}_{\mathrm{g}}$ and $1^{1} \mathrm{~B}_{1 \mathrm{u}}$ states of ethene and the corresponding excitation energies. We employ the ANO double- $\zeta$ basis sets with the augmentations listed in Table 7.1, and the triple- $\zeta$ basis with and without $+\mathrm{C}(\mathrm{s}, \mathrm{p}, \mathrm{d})$ augmentation.

In all FCIQMC calculations, we find that the number of walkers needed to converge the $1^{1} \mathrm{~B}_{1 \mathrm{u}}$ state is many orders of magnitude smaller than the dimensions of the FCI space. For example, we obtain convergence with a population of approximately $10^{8}$ walkers in a FCI space of $10^{18}$ determinants in correspondence of the largest VTZP+C(s,p,d) basis set. We also find that the size of the walker population scales with respect to the FCI dimension according to a power law (Eq. 7.4) as previously observed for other systems. However, as shown in Figure 7.1, the scaling of the augmented basis sets constructed from the ANO double- $\zeta$ basis differs significantly from that of triple- $\zeta$ basis sets. For instance, the VTZP basis set without augmentation requires the same number of walkers as the VDZP+all basis even though its FCI space is almost 100 times larger. Therefore, the triple- $\zeta$ basis sets appear to require fewer walkers than what the extrapolation from the augmented double- $\zeta$ basis sets would suggest. We believe that this difference of prefactors stems from the higher relative number of diffuse basis functions in the ANO double basis set series as compared to the two triple- $\zeta$ basis sets considered here. Indeed, once the augmentation functions are added, it is found that the $\pi^{*}$ orbital no longer corresponds to the Hartree-Fock LUMO orbital. This potentially has significant implications as regards the rapidity of convergence of the FCI wave function of both the $A_{g}$ state and the $1^{1} B_{1 u}$ states, since the appearance of additional low energy determinants may result in a significantly more multi-configurational wave function. This undesirable behavior may be ameliorated by employing a different one-electron basis, but in this study we continue to use a Hartree-Fock basis, leaving this question for a future study. In general, however, we find that the introduction of very diffuse functions leads to a slower convergence of the FCIQMC simulation. A log-log fit of only the double- $\zeta$ populations yields $\alpha=0.48$. It is unclear from only two basis sets whether the triple- $\zeta$ basis sets have the same scaling parameter $\alpha$. The scaling parameter of ethene is therefore larger than the ones obtained for first and second row atoms and anions ( $\alpha=0.16)$ [34] or $\mathrm{C}_{2}(\alpha=0.34)$ [35]. Clearly, the size of the FCI space is not the only relevant parameter which affects the size of the required walker population, but the relative number and the diffuseness of the augmented functions also need to be taken into account.

The FCIQMC excitation energies display a smooth convergence with respect 


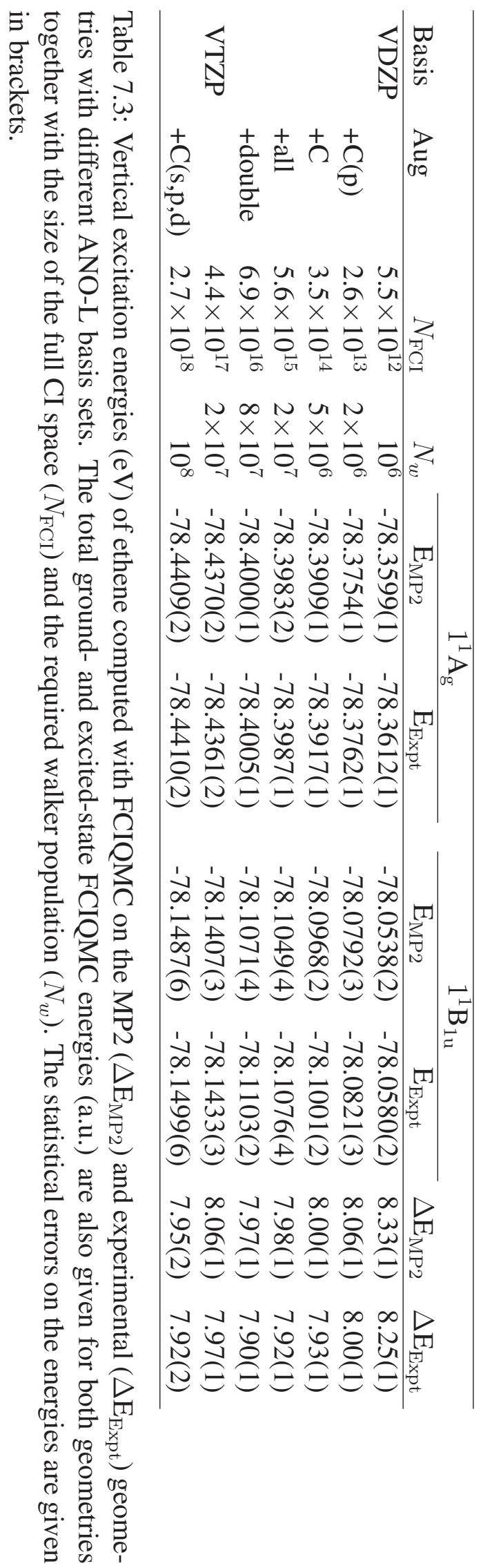




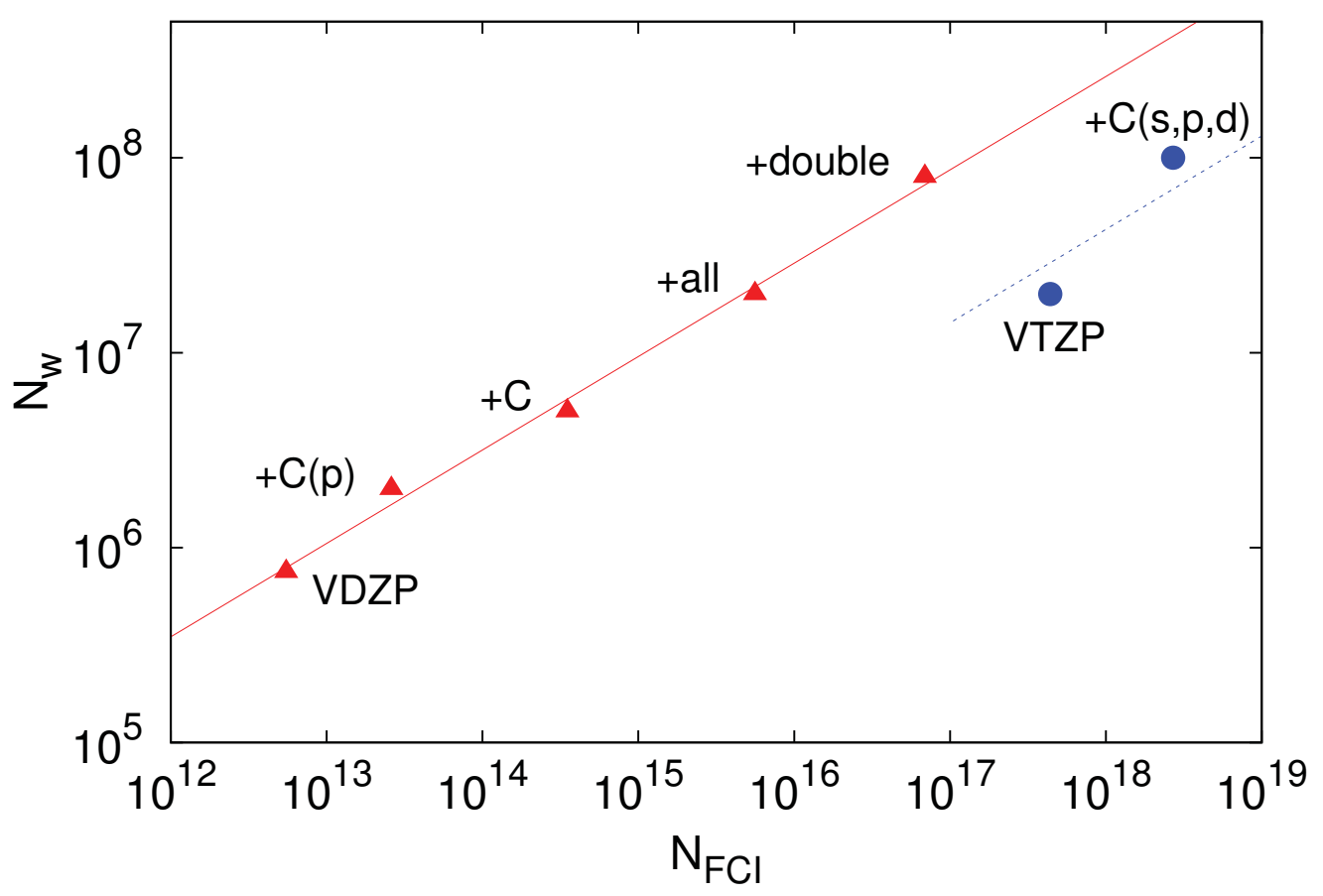

Figure 7.1: Logarithmic plot of the dimension of the FCI space $\left(N_{\mathrm{FCI}}\right)$ and the corresponding walker population $\left(N_{w}\right)$ employed in the calculations of the $1^{1} \mathrm{~B}_{1 \mathrm{u}}$ state of ethene for five basis sets based on VDZP (red triangles) and two basis sets based on VTZP (blue circles). A log-log fit of the excitations computed with augmented VDZP basis sets yields a slope of $\alpha=0.48$ (solid, red line). The blue dashed line, which is drawn with the same slope as the red line, suggests that the VTZP basis sets might display similar scaling properties.

to the basis sets of the augmented ANO double- $\zeta$ series. The VDZP+double values computed on the MP2 and the experimental geometry are equal to 7.97(1) and 7.90(1) eV, respectively, and compatible with the corresponding excitation energies of 7.95(2) and 7.92(2) eV obtained with the VTZP+C(s,p,d) basis set. To assess the degree of convergence of these excitation energies with basis set, we compare the FCIQMC results with the CCSD, CC3, and CCSDT excitation energies computed with much larger basis sets of up to quintuple- $\zeta$ quality.

The coupled-cluster excitations are compared with the FCIQMC values in Table 7.4, and the convergence of the excitations with basis set is illustrated in Figure 7.2. The CC3 and CCSDT values are very close to the FCIQMC energies and the $\mathrm{CC} 3$ results computed with the $\mathrm{VDZP}+$ double and the $\mathrm{VTZP}+\mathrm{C}(\mathrm{s}, \mathrm{p}, \mathrm{d})$ basis sets are converged to better than 0.01 and $0.02 \mathrm{eV}$, respectively. Given the very similar behavior of the coupled-cluster and FCIQMC excitations as a function of basis set, it is safe to expect that also the FCIQMC excitation energies computed with the VDZP+double basis set are affected by a similar, small basis-set error of at most $0.01 \mathrm{eV}$. If we correct the FCIQMC/VDZP+double values by this amount, we esti- 


\begin{tabular}{llcccc}
\hline Basis & Aug & CCSD & CC3 & CCSDT & FCIQMC \\
\hline VDZP & & $8.44 / 8.37$ & $8.33 / 8.26$ & $8.34 / 8.26$ & $8.33(1) / 8.25(1)$ \\
& + C(p) & $8.13 / 8.07$ & $8.05 / 7.99$ & $8.06 / 8.00$ & $8.06(1) / 8.00(1)$ \\
& + C & $8.08 / 8.02$ & $7.98 / 7.92$ & $7.99 / 7.93$ & $8.00(1) / 7.93(1)$ \\
& +all & $8.06 / 8.00$ & $7.95 / 7.89$ & $7.96 / 7.90$ & $7.98(1) / 7.92(1)$ \\
& +double & $8.03 / 7.97$ & $7.93 / 7.87$ & $7.94 / 7.88$ & $7.97(1) / 7.90(1)$ \\
VTZP & & $8.15 / 8.08$ & $8.03 / 7.96$ & $8.04 / 7.97$ & $8.06(1) / 7.97(1)$ \\
& + +C(s,p,d) & $8.06 / 7.99$ & $7.94 / 7.88$ & $7.96 / 7.89$ & $7.95(2) / 7.92(2)$ \\
& +all & $8.05 / 7.99$ & $7.93 / 7.87$ & $7.95 / 7.88$ & \\
& + double & $8.05 / 7.98$ & $7.93 / 7.86$ & $7.94 / 7.87$ & \\
VQZP & & $8.06 / 7.99$ & $7.94 / 7.88$ & $7.96 / 7.89$ & \\
& $+C$ & $8.05 / 7.98$ & $7.93 / 7.86$ & & \\
& + +all & $8.05 / 7.98$ & $7.93 / 7.86$ & & \\
V5ZP & & $8.05 / 7.99$ & $7.93 / 7.87$ & & \\
\hline
\end{tabular}

Table 7.4: Vertical excitation energies (eV) of ethene calculated with CCSD, CC3, CCSDT, and FCIQMC and different ANO-L basis sets on the MP2 and the experimental geometry (separated by a slash as MP2/Expt). The statistical errors on the FCIQMC excitation energies are given in brackets.

mate that the vertical excitation energy of ethene is $\Delta \mathrm{E}_{\mathrm{MP} 2}=7.96(1) \mathrm{eV}$ on the MP2 geometry and $\Delta \mathrm{E}_{\mathrm{EXP}}=7.89(1) \mathrm{eV}$ on the experimental geometry.

While all FCIQMC calculations above have been obtained treating all possible excitations of the 12 valence electrons, it is interesting to find out which level of excitation plays an important role in our final results. Therefore, for the VDZP+all basis set which has proven to yield nearly converged results for ethene, we compute the excitation energies with excitations limited to 3, 4, 5, 6 and 8 electrons. The walker population is kept fixed at the size needed at the FCI level, namely, $2 \times 10^{7}$. The $1^{1} \mathrm{~A}_{\mathrm{g}}$ state is already converged with an excitation level of 5 , but the $1^{1} \mathrm{~B}_{1 \mathrm{u}}$ state needs at least sixfold excitations as shown in Table 7.5.

\begin{tabular}{cccc}
\hline Max exc. & $1^{1} A_{g}$ & $1^{1} B_{1 u}$ & $\Delta \mathrm{E}$ \\
\hline 3 & $-78.3700(0)$ & $-78.0450(1)$ & $8.84(0)$ \\
4 & $-78.3973(1)$ & $-78.0948(2)$ & $8.23(1)$ \\
5 & $-78.3980(1)$ & $-78.1027(3)$ & $8.04(1)$ \\
6 & $-78.3983(1)$ & $-78.1051(3)$ & $7.98(1)$ \\
8 & $-78.3983(1)$ & $-78.1053(3)$ & $7.97(1)$ \\
12 & $-78.3983(2)$ & $-78.1049(2)$ & $7.98(1)$ \\
\hline
\end{tabular}

Table 7.5: FCIQMC total (a.u.) and vertical excitation (eV) energies of ethene computed with limited excitations. We employ the MP2 ground-state equilibrium geometry, the VDZP+all basis set, and a walker population of $5 \times 10^{6}$. A maximum excitation (Max exc.) of 12 corresponds to a (frozen-core) FCI calculation. The statistical error on the energies is given in brackets. 


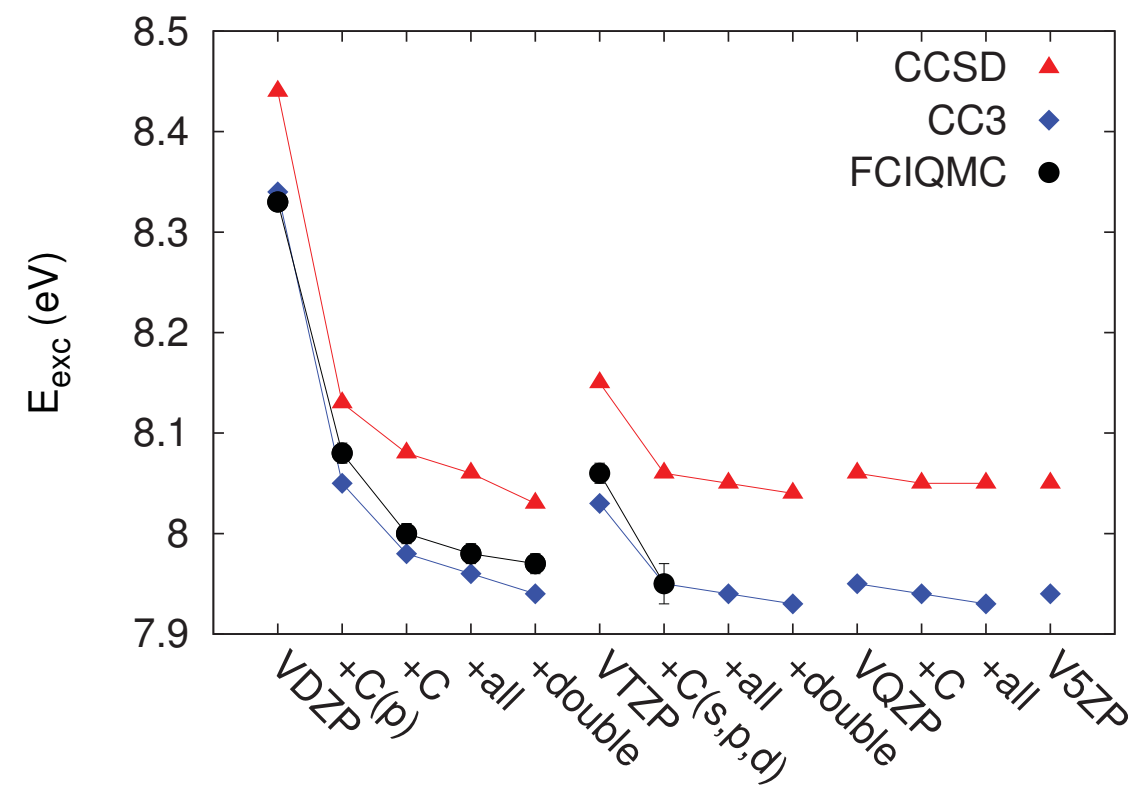

Figure 7.2: Basis-set convergence of the excitation energies (eV) of ethene, computed with CCSD, CC3, and FCIQMC on the MP2 geometry. A very similar behavior is observed on the experimental geometry. The statistical error bar on the FCIQMC energies is either smaller than the size of the symbols or is indicated.

To further validate our FCIQMC results, we investigate two possible sources of error in our calculations, that is, the frozen-core approximation and the lack of additional diffuse functions. In all the FCIQMC calculations above, we have frozen the lowest four orbitals corresponding to the $1 \mathrm{~s}$ electrons of the carbon atoms. We therefore unfreeze all orbitals in the computation of the excitation energy of ethene computed with the VDZP+all basis set. As expected, the absolute ground- and excitedstate energies change significantly but the vertical excitation energy of 7.97(1) remains compatible with the value of 7.98(1) previously obtained within the frozencore approximation as shown in Table 7.6. Interestingly, while the FCI space increases by a factor of $10^{4}$, the required population is only 2.5 times larger than in the frozen-core calculations. This can be explained with the fact that the determinants corresponding to excitations from the core have significantly higher energies and a relatively small weight, so the walker population needed to map this region of the FCI space is small relative to the total number of determinants. We note that the CCSD and $\mathrm{CC} 3$ excitations computed without the frozen-core approximation also change by at most $0.01 \mathrm{eV}$ (data not shown).

As for the diffuseness of the basis, several previous calculations of the vertical excitation energy of ethene employ basis sets which are more diffuse than the ones considered in this work. In particular, the two investigations which have led to the lowest estimate of the vertical excitation at $7.7 \mathrm{eV}[7,13]$ have used more diffuse functions on the carbon atoms. Therefore, to verify that such diffuse functions do 


\begin{tabular}{cccccc}
\hline Orb. frozen & $N_{\mathrm{FCI}}$ & $N_{w}$ & $\mathrm{E}\left(1^{1} \mathrm{~A}_{\mathrm{g}}\right)$ & $\mathrm{E}\left(1^{1} \mathrm{~B}_{1 \mathrm{u}}\right)$ & $\Delta \mathrm{E}$ \\
\hline 0 & $7.9 \times 10^{19}$ & $5 \times 10^{7}$ & $-78.4121(1)$ & $-78.1193(2)$ & $7.97(1)$ \\
4 & $5.6 \times 10^{15}$ & $2 \times 10^{7}$ & $-78.3983(2)$ & $-78.1049(2)$ & $7.98(1)$ \\
\hline
\end{tabular}

Table 7.6: FCIQMC total ( $\mathrm{E}$ in a.u.) and vertical excitation $(\Delta \mathrm{E}$ in $\mathrm{eV})$ energies of ethene computed with and without frozen orbitals. The VDZP+all basis set and the MP2 equilibrium geometry are used. The statistical error on the energies is given in brackets.

\begin{tabular}{lccccc}
\hline Basis & $N_{\mathrm{FCI}}$ & $N_{w}$ & $\mathrm{E}\left(1^{1} \mathrm{~A}_{\mathrm{g}}\right)$ & $\mathrm{E}\left(1^{1} \mathrm{~B}_{\mathrm{u}}\right)$ & $\Delta \mathrm{E}$ \\
\hline VDZP & $2.5 \times 10^{25}$ & $3 \times 10^{8}$ & $-155.5481(6)$ & $-155.3072(4)$ & $6.56(2)$ \\
& & $10^{9}$ & $-155.5491(4)$ & $-155.3092(6)$ & $6.53(2)$ \\
VTZP $^{\prime}$ & $1.1 \times 10^{29}$ & $10^{9}$ & $-155.6076(4)$ & $-155.373(1)$ & $6.38(3)$ \\
\hline
\end{tabular}

Table 7.7: Vertical excitation energies $(\Delta \mathrm{E}$ in $\mathrm{eV})$ of butadiene computed with FCIQMC with different ANO-L basis sets. The total ground- and excited-state FCIQMC energies (a.u.) are also given together with the size of the full CI space $\left(N_{\mathrm{FCI}}\right)$ and the required walker population $\left(N_{w}\right)$. The statistical error on the energies is given in brackets.

not change the excitation energy, we further augment our VQZP+all basis set with the p-augmentation of carbon from Ref. 13, by adding two sets of Gaussians with exponents 0.010727 and 0.003576 (in total, twelve additional spatial basis functions). The resulting basis set is not the same as the one used in Refs. [7] or [13] because ANO-L-VQZP has more primitives than cc-pVQZ, but the total number of functions is the same and the diffuse functions are identical. At the CCSD and CC3 level, we find that the additional diffuse functions have very little effect on the excitation energies which are only $0.01 \mathrm{eV}$ lower than the values obtained with the VQZP+all basis.

Finally, we note that the ground-state FCIQMC energies in Table 7.3 should also give us an indication of the quality of the MP2 equilibrium structure as compared to the experimental geometry from Ref. 57. We first observe that the ground-state FCIQMC energies obtained with the VDZP basis set are lower on the experimental geometry than on the MP2 one. However, this difference diminishes as the basis is enlarged to the VDZP+double and the situation reverses at the triple- $\zeta$ level. This observation is consistent with our findings in optimizing the equilibrium geometry with different basis sets, namely, that a small basis of double valence quality favors a longer carbon-carbon bond length as the one in the experimental geometry (see Table 7.2).

\subsubsection{Butadiene}

For butadiene, we perform FCIQMC calculations with two basis sets without diffuse functions, namely, VDZP and $\mathrm{VTZP}^{\prime}$, and report the total and excitation energies in Table 7.7. For the VDZP basis set, we obtain a converged excitation energy of 
$6.56(2) \mathrm{eV}$ with a population of $3 \times 10^{8}$ walkers, and further increasing the population to $10^{9}$ walkers leads to a ground-state energy compatible within statistical error and only a slightly lower excited-state energy, reducing the excitation energy to 6.53(2) $\mathrm{eV}$. The VTZP' basis yields a FCI space of $10^{29}$ determinants and requires instead populations of at least $10^{9}$ walkers, which is computationally quite demanding and at the limits of what we can afford. With this population size, we achieve convergence for the ground-state energy but cannot further increase the number of walkers to verify convergence of the excited state.

Nevertheless, we believe that the corresponding excitation energy in the VTZP' basis set of 6.38(3) eV is nearly converged to the FCI limit for two reasons. First, halving the number of walkers yields a compatible ground-state energy within a statistical error of $1 \mathrm{mHartree}$, and raises the excited-state energy by less than $3 \pm 1$ mHartree. The corresponding excitation energy is increased by $0.10 \pm 0.05 \mathrm{eV}$. The convergence behavior for the two basis sets without diffuse functions in ethene and for the VDZP basis in butadiene reveals that the excited state requires a walker population less or equal to roughly twice the population needed for the ground state. Since we obtain convergence for the ground state of butadiene in the VTZP' basis set with $5 \times 10^{8}$ walkers, we do not expect that a further increase of the population beyond $10^{9}$ for the excited state will significantly affect the excitation energy. Second, we can assess convergence of our FCIQMC total and excitation energies through a comparison with the CCSD, CC3, and CCSDT values. As shown in Table 7.9 and in Figure 7.3, our FCIQMC excitation energies appear to have a similar convergence behavior as the $\mathrm{CC}$ values. For both basis sets, they lie in the range bordered on the high side by the CCSD and on the low side by the CCSDT or CC3 values, being slightly closer to CCSD. The difference of $0.15 \mathrm{eV}$ between the FCIQMC excitation energies computed with the two basis sets is comparable to what is obtained within CC. Furthermore, if we compare the total ground- and excited-state FCIQMC energies with the corresponding frozen-core CCSD values, we find that FCIQMC gives very similar shifts with respect to CC for both the VDZP and VTZP' basis sets, as shown in Table 7.8. These facts lead us to conclude that the employed walker population of $10^{9}$ is close to sufficient for the $\mathrm{VTZP}^{\prime}$ basis set.

To estimate the remaining basis set error, we note that the $\mathrm{CC}$ results are converged when the $\mathrm{VTZP}+\mathrm{C}(\mathrm{p})$ and larger basis sets are employed, and that $\mathrm{VTZP}^{\prime}$ yields $\mathrm{CC}$ excitations which are only $0.06 \mathrm{eV}$ higher than the converged values. Given the similar convergence behavior of FCIQMC, we expect that the basis-set correction should not be significantly different from the $0.06 \mathrm{eV}$ value estimated within $\mathrm{CC}$. With the use of this correction, we assign a value of $\Delta \mathrm{E}=6.32(3) \mathrm{eV}$ to the vertical excitation of butadiene.

Finally, we note that, as in the case of ethene, the use of basis sets with diffuse functions slows down the convergence of the FCIQMC energies, and leads to even larger walker populations, especially for the $1^{1} \mathrm{~B}_{\mathrm{u}}$ state. For instance, even though the VDZP+C and VTZP' basis sets are characterized by exactly the same size of FCI space, the excited state shows a significantly slower convergence with population size for the $\mathrm{VDZP}+\mathrm{C}$ basis. In particular, the $\mathrm{VDZP}+\mathrm{C}$ excited-state energy computed with a population of $10^{9}$ walkers is significantly closer to the CCSD value than 


\begin{tabular}{|c|c|c|c|c|}
\hline \multirow[b]{2}{*}{ Basis } & \multirow[b]{2}{*}{ Aug } & \multicolumn{3}{|c|}{$E\left(1^{1} A_{g}\right)$} \\
\hline & & FCIQMC & CCSD & $\Delta \mathrm{E}$ \\
\hline \multirow[t]{3}{*}{ ANO-L-VDZP } & & $-155.5481(3)$ & -155.5332 & $-0.0149(3)$ \\
\hline & $+\mathrm{C}(\mathrm{p})$ & $-155.5711(6)$ & -155.5566 & $-0.0145(6)$ \\
\hline & $+\mathrm{C}$ & $-155.5993(5)$ & -155.5838 & $-0.0155(5)$ \\
\hline \multirow[t]{2}{*}{ ANO-L-VTZP' } & & $-155.6076(1)$ & -155.5931 & $-0.0145(1)$ \\
\hline & & \multicolumn{3}{|c|}{$\mathrm{E}\left(1^{1} \mathrm{~B}_{\mathrm{u}}\right)$} \\
\hline Basis & Aug & FCIQMC & CCSD & $\Delta \mathrm{E}$ \\
\hline ANO-L-VDZP & & $-155.3072(1)$ & -155.2902 & $-0.0170(1)$ \\
\hline & $+\mathrm{C}(\mathrm{p})$ & $-155.3343(5)$ & -155.3203 & $-0.0140(5)$ \\
\hline & $+\mathrm{C}$ & $-155.3606(11)$ & -155.3490 & $-0.0116(11)$ \\
\hline ANO-L-VTZP' & & $-155.3731(6)$ & -155.3574 & $-0.0157(6)$ \\
\hline
\end{tabular}

Table 7.8: Absolute energies (a.u.) of the $1^{1} \mathrm{~A}_{\mathrm{g}}$ and $1^{1} \mathrm{~B}_{\mathrm{u}}$ states of butadiene computed with CCSD and FCIQMC with four ANO basis sets. The difference between FCIQMC and CCSD energies ( $\triangle \mathrm{E}$, a.u.) is also given.

\begin{tabular}{llcccc}
\hline Basis & Aug & CCSD & CC3 & CCSDT & FCIQMC \\
\hline VDZP & & 6.61 & 6.48 & 6.50 & $6.53(2)$ \\
& $+\mathrm{C}(\mathrm{p})$ & 6.43 & 6.32 & 6.34 & \\
& $+\mathrm{C}$ & 6.39 & 6.27 & 6.29 & \\
& + all & 6.38 & 6.26 & 6.27 & \\
VTZP $^{\prime}$ & & 6.41 & 6.29 & 6.31 & $6.38(3)$ \\
VTZP & & 6.39 & 6.26 & 6.28 & \\
& $+\mathrm{C}(\mathrm{p})$ & 6.36 & 6.24 & 6.25 & \\
& $+\mathrm{C}(\mathrm{s}, \mathrm{p}, \mathrm{d})$ & 6.36 & 6.23 & & \\
& $+\mathrm{all}$ & 6.35 & & & \\
VQZP & & 6.35 & & & \\
& $+\mathrm{C}(\mathrm{p})$ & 6.35 & & & \\
\hline
\end{tabular}

Table 7.9: Vertical excitation energies $(\mathrm{eV})$ of butadiene calculated with coupled cluster and FCIQMC. The statistical error on the energies is given in brackets. 


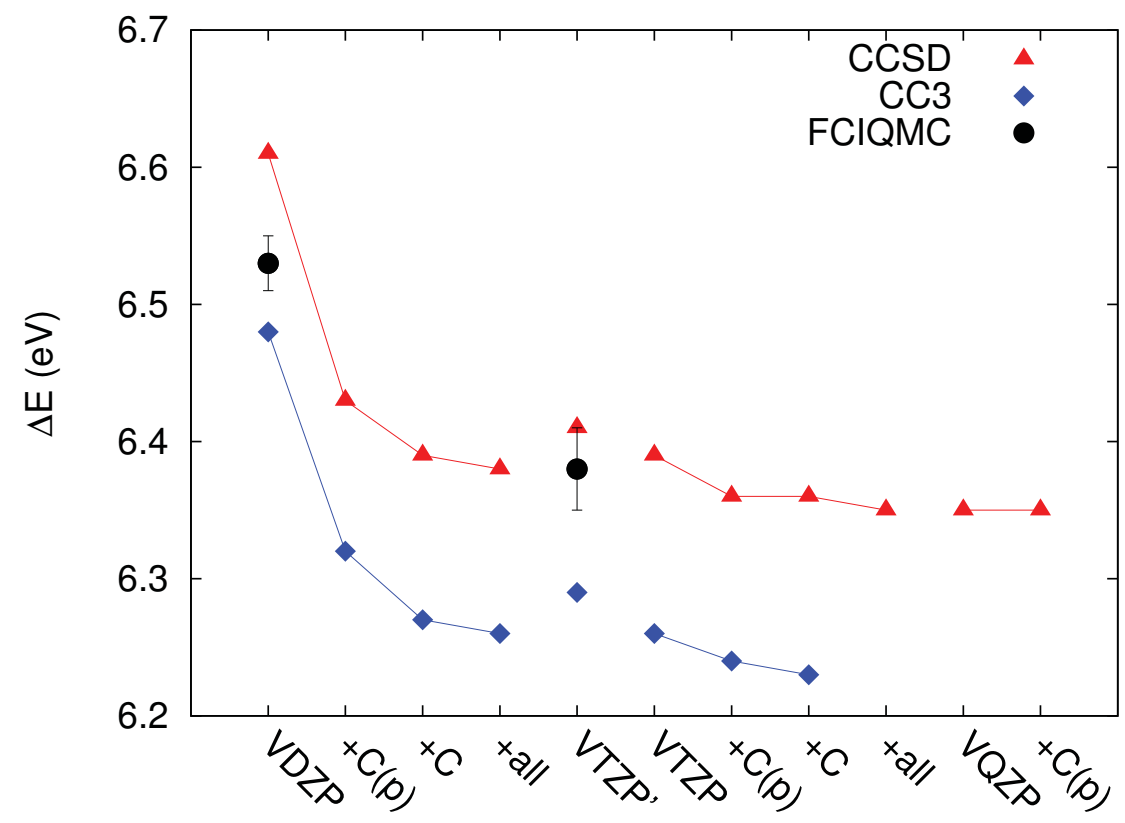

Figure 7.3: Basis-set convergence of the vertical excitation energies $(\mathrm{eV})$ of butadiene, computed with CCSD, CC3, and FCIQMC.

what is observed for the VDZP and VTZP' basis sets, and therefore too high (see Table 7.8).

\subsubsection{Theoretical comparison}

In Table 7.10, we collect the most relevant calculations of the vertical excitation energy of ethene from the literature, and compare them with our FCIQMC estimates of 7.96(1) and 7.89(1) eV on the MP2/cc-pVQZ and the experimental geometry of Ref. 57, respectively. We note that the listed calculations have been performed mainly on the same experimental geometry or on a geometry characterized by a carbon-carbon bond length between our MP2 (1.330 $\AA$ ) and the experimental value $(1.339 \AA)$. Therefore, these calculations can be either directly compared with our FCIQMC excitation on the same experimental structure or with a reference value which should lie between the FCIQMC estimates of 7.89(1) and 7.96(1) eV obtained on the experimental and MP2 structures. Multi-state (MS) CASPT2 calculations $[5,12,15]$ and previous CC calculations up to perturbative third order $[4,12]$ give excitations in the range of 7.8-8.0 eV. At variance with these calculations, an excitation energy of $7.7 \mathrm{eV}$ is obtained with either multi-reference (MR) configuration interaction and coupled cluster methods on appropriately constructed molecular orbitals [7], or with the perturbative NEVPT2 approach with the use of orbitals obtained with restricted active space methods [13]. Therefore, the CASPT2 and CC results are compatible with our FCIQMC excitation energies, while the value of 7.7 $\mathrm{eV}$ reported in Refs. $[7,13]$ is significantly lower. We reiterate that this difference 
does not stem from the choice of geometry since we employ the same experimental structure, nor from the use of more diffuse augmented functions, which only affects the excitation energy by $0.01 \mathrm{eV}$ as shown above. It is rather difficult to establish why Refs. [7, 13] obtain such a low excitation. In our FCIQMC calculations, there is no need to artificially constrain the available excitations to an appropriate set of orbitals as in the RASSCF and subsequent NEVPT2 calculations of Ref. 13 and sizeextensivity is guaranteed, unlike the MR-CISD+Q calculations of Ref. 7, which are based on the Davidson correction [60] on CISD values. In fact, bare MR-CISD with the largest CAS reference gives an excitation energy of $7.57 \mathrm{eV}$, which means that a Davidson correction of $0.12 \mathrm{eV}$ is significant and that the excitation energy is likely to become larger when higher excitations are included in the CI expansion.

Table 7.11 reports a similar comparison for the vertical excitation energy of butadiene. Several calculations in the literature compare rather well with our FCIQMC estimate of 6.32(3) eV. Previous CC calculations yield excitation energies in the range of 6.2-6.4 eV [4,22] with CC3 and CCSD being at the low and the high side of this range, respectively. The single-state (SS) CASPT2 and RASPT2 excitation energies of about $6.3 \mathrm{eV}$ [14] are in good agreement with our FCIQMC estimate while the CASPT2 value of $6.0 \mathrm{eV}$ [18] is significantly red-shifted, possibly due to the use of a different zero-order Hamiltonian. It is hard to establish the quality of the CASPT2 calculation of Ref. 3 due to the lack of multi-state treatment in combination with the use of diffuse functions which induce valence-Rydberg mixing in the excited state. The MR-CISD+Q result of $6.34 \mathrm{eV} \mathrm{[20]} \mathrm{is} \mathrm{also} \mathrm{in} \mathrm{very} \mathrm{good} \mathrm{agreement} \mathrm{with} \mathrm{our}$ FCIQMC excitation energy but their MR-AQCC value of $6.18 \mathrm{eV}$ is too red-shifted. In comparing the excitation energies of Table 7.11, we must however keep in mind that they are computed with different geometries and basis sets, and that the quality of the basis sets is less uniform than in the previous comparison for ethene. For instance, our CC calculations show that the VDZP basis employed in Ref. 18 is clearly inadequate. On the other hand, we expect that the geometrical differences affect to a lesser degree the corresponding vertical excitations. From Table 7.2, we can see for example that our MP2/cc-pVQZ geometry gives the same excitation energy at the CCSD level as the experimental geometry [53] employed in Refs. [3,4]. Similarly, our CC results agree within 0.01-0.04 eV with the ones of Ref. 22, where yet another geometry, optimized with the MP2 method but with a TZ-VPP basis set, is used.

Finally, we compute a weighted average value of the absorption spectrum of butadiene [28], namely, $\int I \mathrm{~d} E / \int(I / E) \mathrm{d} E$, as proposed in Ref. 6 for ethene, and obtain a value of 5.95-6.00 eV, depending on the limits of integration. This estimate is a better estimate of the vertical excitation than the band maximum because it takes into account adiabatic Franck-Condon coupling. However, it is still significantly lower than our FCIQMC excitation energy and most other values in the literature, indicating that non-adiabatic couplings to other excited states are important. 


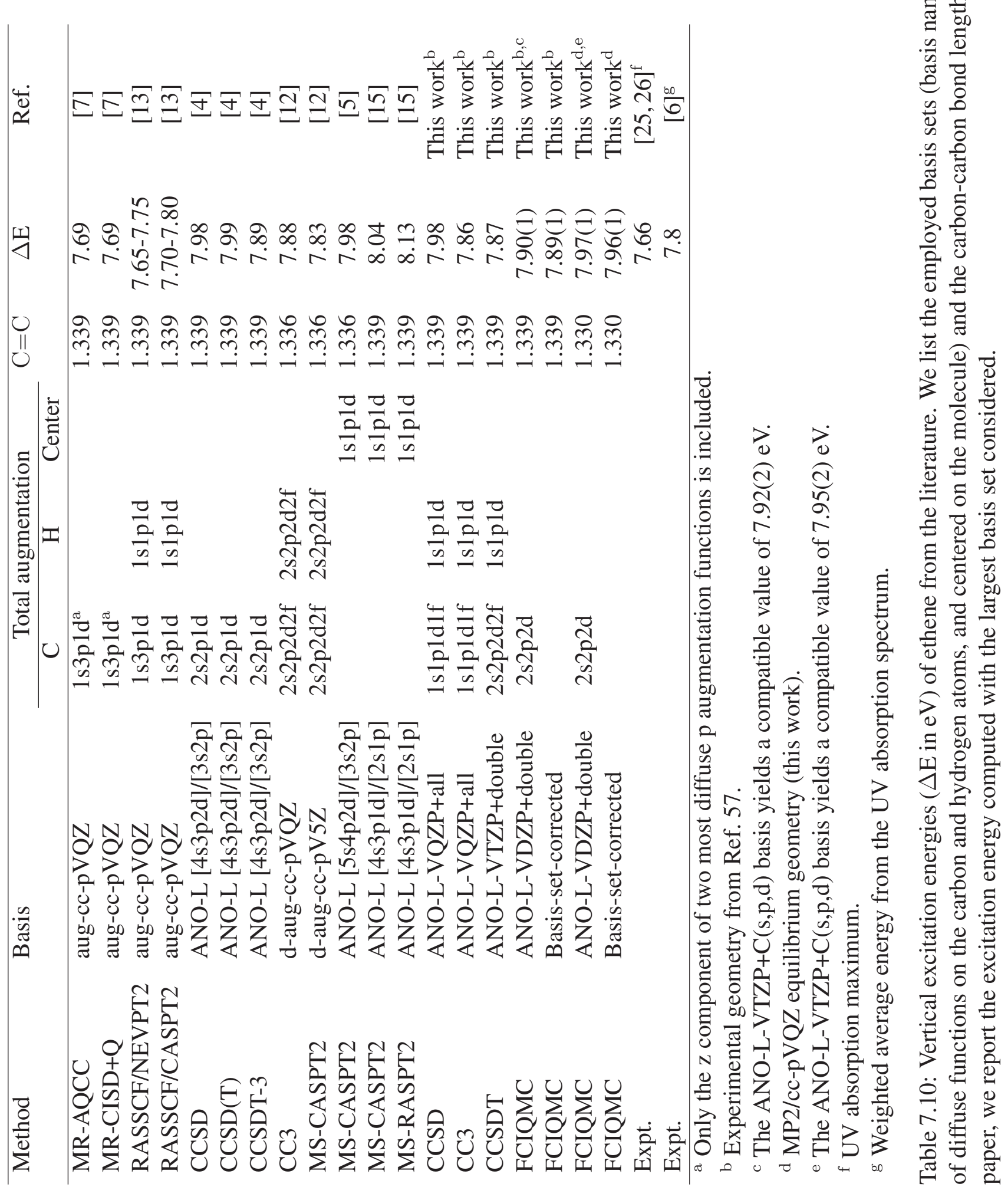




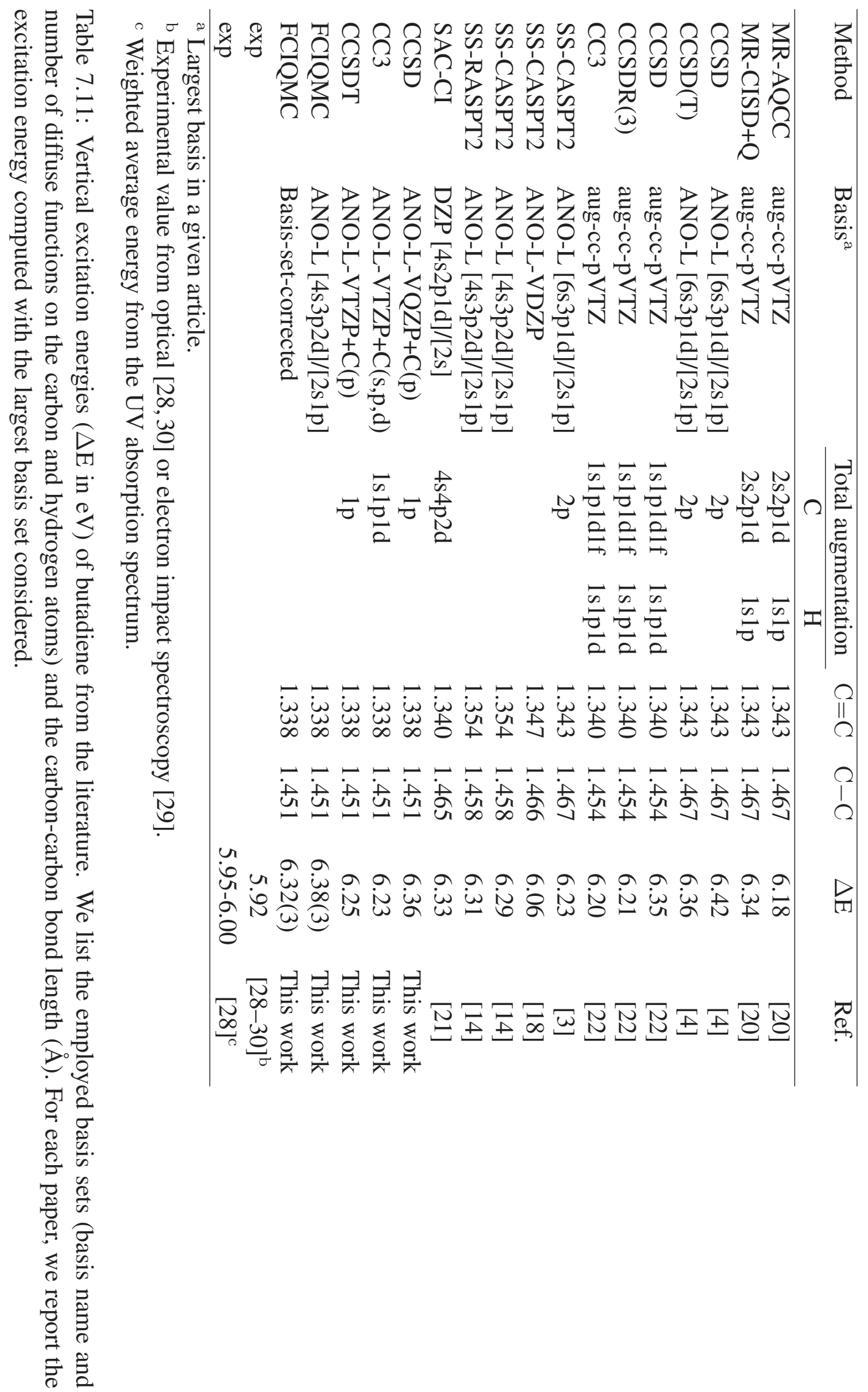




\subsection{Conclusions}

In this work, we employ the recently developed FCIQMC method to compute the $\pi \rightarrow \pi^{*}$ vertical excitation energies of ethene and butadiene, which have been the subject of extensive theoretical investigation for more than two decades and for which reliable estimates are still needed. For this complex problem, FCIQMC allows us to treat remarkably large FCI spaces which would be unthinkable for conventional FCI, namely, $10^{18}$ determinants for ethene and $10^{29}$ determinants for butadiene corresponding to a partially augmented triple-valence basis and a partial triple-valence basis, respectively.

For ethene, we obtain a vertical excitation energy in the range of 7.89-7.96 eV, depending on the particular equilibrium ground-state geometry employed. These values correspond to the FCIQMC limit for a double-augmented ANO-L basis set of double- $\zeta$ quality corrected for a basis-set error of $0.01 \mathrm{eV}$ estimated within $\mathrm{CC}$. The analysis of the FCIQMC excitations computed with a series of basis sets up to a partially augmented ANO-L triple-valence basis confirms our extrapolation. The vertical excitation energy is therefore definitely higher than the experimental absorption band maximum located at $7.66 \mathrm{eV}$, indicating that nonadiabatic effects are important and responsible for shifting the location of the vertical excitation to higher energies. For butadiene, the FCIQMC computation of the excitation is significantly more challenging even though the approach is able to reduce the complexity of the problem by 12 orders of magnitude with respect to conventional FCI. With an ANO-L triplevalence basis on the carbon atoms, we are close to convergence to the FCIQMC limit with populations of $10^{9}$ walkers but going to larger populations is currently beyond our computational possibilities. Our best estimate is a vertical excitation of about $6.3 \mathrm{eV}$, which we obtain with the use of a partial ANO-L triple-valence basis and a basis-set correction of $0.06 \mathrm{eV}$ estimated within $\mathrm{CC}$. This value is significantly blue-shifted with respect to the location of the absorption band maximum at 5.92 $\mathrm{eV}$, indicating the importance of nonadiabatic effects also in butadiene. Our recommended estimates of the vertical excitation energies of both molecules represent a robust, reliable benchmarking reference for future calculations. 
7 Full $\mathrm{Cl}$ excitations of ethene and butadiene: Resolution of an ancient question 


\section{Bibliography}

[1] C. Petrongolo, R. J. Buenker, and S. D. Peyerimhoff, J. Chem. Phys. 76, 3655 (1982).

[2] R. Lindh and B. O. Roos, Int. J. Quantum Chem. 813 (1989).

[3] L. Serrano-Andrés, M. Merchan, I. Nebot-Gil, R. Lindh, and B. O. Roos, J. Chem. Phys. 98, 3151 (1993).

[4] J. D. Watts, S. R. Gwaltney, and R. J. Bartlett, J. Chem. Phys. 105, 6979 (1996).

[5] J. Finley, P.-Å. Malmqvist, B. O. Roos, and L. Serrano-Andrés, Chem. Phys. Lett. 288, 299 (1998).

[6] E. R. Davidson and A. A. Jarzecki, Chem. Phys. Lett. 285, 155 (1998).

[7] T. Müller, M. Dallos, and H. Lischka, J. Chem. Phys. 110, 7176 (1999).

[8] M. Ben-Nun and T. J. Martinez, Chem. Phys. 259, 237 (2000).

[9] K. K. Baeck and T. J. Martinez, Chem. Phys. Lett. 375, 299 (2003).

[10] A. Hazra, H. H. Chang, and M. Nooijen, J. Chem. Phys. 121, 2125 (2004).

[11] F. Schautz and C. Filippi, J. Chem. Phys. 120, 10931 (2004).

[12] M. Schreiber, M. R. Silva-Junior, S. P. A. Sauer, and W. Thiel, J. Chem. Phys. 128, 134110 (2008).

[13] C. Angeli, J. Comput. Chem. 30, 1319 (2009).

[14] A. R. M. Shahi, C. J. Cramer, and L. Gagliardi, Phys. Chem. Chem. Phys. 11, 10964 (2009).

[15] V. Sauri, L. Serrano-Andrés, A. R. M. Shahi, L. Gagliardi, S. Vancoillie, and K. Pierloot, J. Chem. Theory Comp. 7, 153 (2011).

[16] R. J. Cave and E. R. Davidson, Chem. Phys. Lett. 148, 190 (1988).

[17] P. Cronstrand, P. Christiansen, P. Norman, and H. Agren, Phys. Chem. Chem. Phys. 3, 2567 (2001). 
[18] B. Ostojić and W. Domcke, Chem. Phys. 269, 1 (2001).

[19] M. Boggio-Pasqua, M. J. Bearpark, M. Klene, and M. A. Robb, J. Chem. Phys. 120, 7849 (2004).

[20] M. Dallos and H. Lischka, Theor. Chem. Acc. 112, 16 (2004).

[21] B. Saha, M. Ehara, and H. Nakatsuji, J. Chem. Phys. 125, 014316 (2006).

[22] O. Lehtonen, D. Sundholm, R. Send, and M. P. Johansson, J. Chem. Phys. 131, 024301 (2009).

[23] R. Send, D. Sundholm, M. P. Johansson, and F. Pawlowski, J. Chem. Theory Comp. 5, 2401 (2009).

[24] X. Li and J. Paldus, J. Chem. Phys. 134, 214118 (2011).

[25] P. G. Wilkinson and R. S. Mulliken, J. Chem. Phys. 23, 1895 (1955).

[26] R. S. Mulliken, J. Chem. Phys. 66, 2448 (1976).

[27] J.-S. Ryu and B. S. Hudson, Chem. Phys. Lett. 245, 448 (1995).

[28] R. McDiarmid, J. Chem. Phys. 64, 514 (1976).

[29] O. A. Mosher, W. M. Flicker, and A. Kuppermann, J. Chem. Phys. 59, 6502 (1973).

[30] J. P. Doering and R. McDiarmid, J. Chem. Phys. 73, 3617 (1980).

[31] G. H. Booth, A. J. W. Thom, and A. Alavi, J. Chem. Phys. 131, 054106 (2009).

[32] D. Cleland, G. H. Booth, and A. Alavi, J. Chem. Phys. 132, 041103 (2010).

[33] G. H. Booth and A. Alavi, J. Chem. Phys. 132, 174104 (2010).

[34] D. Cleland, G. H. Booth, and A. Alavi, J. Chem. Phys. 134, 024112 (2011).

[35] G. H. Booth, D. Cleland, A. J. W. Thom, and A. Alavi, J. Chem. Phys. 135, 084104 (2011).

[36] E. Rossi, G. L. Bendazzoli, S. Evangelisti, and D. Maynau, Chem. Phys. Lett. 310, 530 (1999).

[37] J. S. Spencer, N. S. Blunt, and W. M. C. Foulkes, J. Chem. Phys. 136, 054110 (2012). 
[38] Y. Shao, L. F. Molnar, Y. Jung, J. Kussmann, C. Ochsenfeld, S. T. Brown, A. T. Gilbert, L. V. Slipchenko, S. V. Levchenko, D. P. O’Neill, R. A. DiStasio Jr, R. C. Lochan, T. Wang, G. J. Beran, N. A. Besley, J. M. Herbert, C. Yeh Lin, T. Van Voorhis, S. Hung Chien, A. Sodt, R. P. Steele, V. A. Rassolov, P. E. Maslen, P. P. Korambath, R. D. Adamson, B. Austin, J. Baker, E. F. C. Byrd, H. Dachsel, R. J. Doerksen, A. Dreuw, B. D. Dunietz, A. D. Dutoi, T. R. Furlani, S. R. Gwaltney, A. Heyden, S. Hirata, C.-P. Hsu, G. Kedziora, R. Z. Khalliulin, P. Klunzinger, A. M. Lee, M. S. Lee, W. Liang, I. Lotan, N. Nair, B. Peters, E. I. Proynov, P. A. Pieniazek, Y. Min Rhee, J. Ritchie, E. Rosta, C. David Sherrill, A. C. Simmonett, J. E. Subotnik, H. Lee Woodcock III, W. Zhang, A. T. Bell, A. K. Chakraborty, D. M. Chipman, F. J. Keil, A. Warshel, W. J. Hehre, H. F. Schaefer III, J. Kong, A. I. Krylov, P. M. W. Gill, and M. Head-Gordon, Phys. Chem. Chem. Phys. 8, 3172 (2006).

[39] M. J. Frisch, G. W. Trucks, H. B. Schlegel, G. E. Scuseria, M. A. Robb, J. R. Cheeseman, G. Scalmani, V. Barone, B. Mennucci, G. A. Petersson, H. Nakatsuji, M. Caricato, X. Li, H. P. Hratchian, A. F. Izmaylov, J. Bloino, G. Zheng, J. L. Sonnenberg, M. Hada, M. Ehara, K. Toyota, R. Fukuda, J. Hasegawa, M. Ishida, T. Nakajima, Y. Honda, O. Kitao, H. Nakai, T. Vreven, J. J. A. Montgomery, J. E. Peralta, F. Ogliaro, M. Bearpark, J. J. Heyd, E. Brothers, K. N. Kudin, V. N. Staroverov, R. Kobayashi, J. Normand, K. Raghavachari, A. Rendell, J. C. Burant, S. S. Iyengar, J. Tomasi, M. Cossi, N. Rega, J. M. Millam, M. Klene, J. E. Knox, J. B. Cross, V. Bakken, C. Adamo, J. Jaramillo, R. Gomperts, R. E. Stratmann, O. Yazyev, A. J. Austin, R. Cammi, C. Pomelli, J. W. Ochterski, R. L. Martin, K. Morokuma, V. G. Zakrzewski, G. A. Voth, P. Salvador, J. J. Dannenberg, S. Dapprich, A. D. Daniels, Ö. Farkas, J. B. Foresman, J. V. Ortiz, J. Cioslowski, and D. J. Fox, Gaussian 09 Revision A.02, Gaussian Inc. Wallingford CT 2009.

[40] F. Aquilante, L. De Vico, N. Ferré, G. Ghigo, P.-Å. Malmqvist, P. Neogrády, T. B. Pedersen, M. Pitoňák, M. Reiher, B. O. Roos, L. Serrano-Andrés, M. Urban, V. Veryazov, and R. Lindh, J. Comput. Chem. 31, 224 (2010).

[41] J. Olsen and P. Jørgensen, J. Chem. Phys. 82, 3235 (1985).

[42] H. Koch and P. Jørgensen, J. Chem. Phys. 93, 3333 (1990).

[43] G. D. Purvis and R. J. Bartlett, J. Chem. Phys. 76, 1910 (1982).

[44] O. Christiansen, H. Koch, and P. Jørgensen, J. Chem. Phys. 103, 7429 (1995).

[45] H. Koch, O. Christiansen, P. Jørgensen, A. M. S. de Merás, and T. Helgaker, J. Chem. Phys. 106, 1808 (1997).

[46] DALTON, a molecular electronic structure program, Release 2.0 (2005), see http://daltonprogram.org/ (accessed July 12, 2012).

[47] K. Kowalski and P. Piecuch, J. Chem. Phys. 115, 643 (2001). 
[48] S. A. Kucharski, M. Wloch, M. Musial, and R. J. Bartlett, J. Chem. Phys. 115, 8263 (2001).

[49] CFOUR, Coupled-Cluster techniques for Computational Chemistry, a quantum-chemical program package by J.F. Stanton, J. Gauss, M.E. Harding, P.G. Szalay with contributions from A.A. Auer, R.J. Bartlett, U. Benedikt, C. Berger, D.E. Bernholdt, Y.J. Bomble, L. Cheng, O. Christiansen, M. Heckert, O. Heun, C. Huber, T.-C. Jagau, D. Jonsson, J. Jusélius, K. Klein, W.J. Lauderdale, D.A. Matthews, T. Metzroth, L.A. Mück, D.P. O’Neill, D.R. Price, E. Prochnow, C. Puzzarini, K. Ruud, F. Schiffmann, W. Schwalbach, S. Stopkowicz, A. Tajti, J. Vázquez, F. Wang, J.D. Watts and the integral packages MOLECULE (J. Almlöf and P.R. Taylor), PROPS (P.R. Taylor), ABACUS (T. Helgaker, H.J. Aa. Jensen, P. Jørgensen, and J. Olsen), and ECP routines by A. V. Mitin and C. van Wüllen. For the current version, see http://www.cfour.de. (accessed July 12, 2012).

[50] T. H. Dunning Jr, J. Chem. Phys. 90, 1007 (1989).

[51] P.-O. Widmark, P.-Å. Malmqvist, and B. O. Roos, Theor. Chim. Acta 77, 291 (1990).

[52] We take the diffuse functions from the aug-cc-pVXZ basis sets in the EMSL Basis Set Library (http://bse.pnl.gov, accessed July 12, 2012).

[53] W. Haugen and M. Trætteberg, Acta Chem. Scand. 20, 1726 (1966).

[54] K. Kuchitsu, T. Fukuyama, and Y. Morino, J. Mol. Struct. 1, 463 (1968).

[55] K. Kveseth, R. Seip, and D. A. Kohl, Acta Chem. Scand., Ser. A 34, 31 (1980).

[56] W. Caminati, G. Grassi, and A. Bauder, Chem. Phys. Lett. 148, 13 (1988).

[57] G. Herzberg, Electronic Spectra of Polyatomic Molecules (Van Nostrand, 1966).

[58] J. L. Duncan, I. J. Wright, and D. V. Lerberghe, J. Mol. Spectr. 42, 463 (1972).

[59] N. C. Craig, P. Groner, and D. C. McKean, J. Phys. Chem. A 110, 7461 (2006).

[60] S. R. Langhoff and E. R. Davidson, Int. J. Quantum Chem. 8, 61 (1974). 


\section{List of publications}

- C. Daday, S. Smart, G. Booth, A. Alavi, and C. Filippi,

"Full Configuration Interaction excitations of ethene and butadiene: Resolution of an ancient question",

J. Chem. Theory Comput. 8, 4441 (2012). (Chapter 7)

- C. Daday, O. Valsson, C. König, J. Neugebauer, and C. Filippi, "State-Specific Embedding Potentials for Excitation-Energy Calculations", J. Chem. Theory Comput. 9, 2355 (2013). (Chapter 3)

- C. Daday, C. König, J. Neugebauer, and C. Filippi, "Wavefunction-in-density functional theory for excited states: Which wavefunctions, which densities?", ChemPhysChem 15, 3205 (2014). (Chapter 4)

- C. Daday, A. Sinicropi, C. Curutchet, B. Mennucci, and C. Filippi, "Chromophore-protein coupling beyond non-polarizable models: Understanding absorption in green fluorescent protein"

Submitted (Chapter 5).

- R. Guareschi, H. Zulfikri, C. Daday, F. M. Floris, C. Filippi, and C. Amovilli, "Introducing QMC/MMpol: Quantum Monte Carlo in polarizable force fields for excited states"

In preparation.

- C. Daday, C. Filippi, A. S. P. Gomes, and C. Jacob, "Wavefunction embedding using exact Freeze\&Thaw cycles" In preparation (Chapter 6). 



\section{Samenvatting}

\section{De Gordiaanse knoop doorhakken in het modeleren van ge- ëxciteerde toestanden in complexe omgevingen}

Dit proefschrift heeft als doelstelling het betrouwbaar en exact modelleren van de lichtabsorptie van realistische biosystemen zoals fluorescente eiwitten (b.v. GFP). Voor dit doel is een multischaal model noodzakelijk vanwege de grootte van dit soort systemen: een eiwit kan duizenden atomen (en dus tienduizenden elektronen) bevatten en een kwantummechanische beschrijving ervan is duidelijk onmogelijk. De lichtabsorptie en elektronexcitatie van deze systemen zijn echter meestal redelijk goed gelokaliseerd binnen het eiwit. Dit gebeurt namelijk op de zogenaamde chromofoor. Een kwantummechanische behandeling van dit beperkte gebied op het eiwit, gecombineerd met een minder accurate maar snellere methode voor de rest van het systeem, is een mogelijke oplossing.

In Hoofdstuk 3 stellen we een nieuw multischaal schema voor, waarin een deel van de omgeving van de chromofoor toch kwantummechanisch behandeld wordt, maar door de rekenkundig minder intensieve dichtheidsfunctionaaltheorie (density functional theory, DFT). Dit soort schema's worden ook golffunctie-in-DFT inbedding (wavefunction-in-DFT embedding, WF/DFT) genoemd en bestonden al voor dit proefschrift, maar onze bedrage is de mogelijkheid van de DFT omgeving om te reageren op de excitatie. Dit schema is getest op een aantal kleine moleculen en wij laten zien dat het meestal een verbetering is op het "bevroren" schema waarin deze reactie niet mogelijk is.

In Hoofdstuk 4 voeren we verdere testen uit op ons schema: we gebruiken meerdere accurate kwantummechanische methodes en variëren verschillende parameters in het schema. Uit onze analyse blijkt dat de belangrijkste factor in ons schema de kinetische energie dichtheidsfunctionaal (kinetic energy density functional, KEDF) is en dat eventuele instabiliteiten of fouten in onze resultaten grotendeels vermeden zouden kunnen worden met de hypothetische "exacte KEDF."

In Hoofdstuk 5 passen we onze nieuwe methode toe op GFP. Wij vergelijken drie verschillende mogelijkheden qua behandeling van de omgeving. Ten eerste beschouwen wij de omgeving als een reeks van elektrische ladingen die niet kunnen reageren op hun omgeving. Ten tweede, de klassieke omgeving krijgt de mogelijkheid om te reageren dankzij polariseerbare dipolen. Ten slotte behandelen wij een deel van de omgeving als een kwantummechanisch onderdeel in de DFT. Uit onze analyse blijkt dat de koppeling tussen de chromofoor en de rest van het eiwit 
een fundamenteel kwantum karakter heeft en de enige manier om excitatie-energieën betrouwbaar te berekenen het gebruik van grote kwantumclusters (d.w.z. zonder multischaal modelering) blijft.

In Hoofdstuk 6 beproeven we een alternatieve vorm van WF/DFT inbouwen, namelijk gebaseerd op "exact embedding." In dit schema wordt er geen gebruik gemaakt van de KEDF's, die de belangrijkste benadering bleken te zijn in Hoofdstuk 4. Wij passen dit schema toe op kleine moleculen die waterstofbruggen vormen met een klein aantal watermoleculen en we bewijzen dat deze methode inderdaad "exact" is in het reproduceren van supermoleculaire elektrondichtheden vanuit berekeningen uitgevoerd op subsystemen. De vergelijking van excitatie-energieën is echter nogal onzeker omdat in ons model de omgevingsreactie op excitatie niet is geïmplementeerd (in tegenstelling tot de schema's in Hoofdstukken 3-5).

Ten slotte, in Hoofdstuk 7 is onze focus op een ander thema dan de rest van het proefschrift: de excitaties van twee kleine moleculen, etheen en butadieen, in geïsoleerde staat. Voor deze toepassing gebruiken we de zogenaamde FCIQMC (volledige configuratie-interactie quantum Monte Carlo, full CI quantum Monte Carlo) methode, die in principe de exacte energieën oplevert zoals de FCI (volledige configuratie-interactie, full $C I$ ) methode dit doet, maar met een aanzienlijk verminderde rekenkundige bewerkingen. Alhoewel dit onderzoek geen gebruik maakt van de eerder genoemde multischaal methodes, past dit hoofdstuk wel in dit proefschrift omdat het aangeeft dat er heel grote verschillen bestaan tussen resultaten van methodes die "betrouwbaar" verondersteld worden. Dit geldt zelfs voor zeer kleine systemen zoals de twee beschreven moleculen met niet meer dan enkele tientallen elektronen (16 en 30, respectievelijk). 


\section{Acknowledgements}

First and foremost, I would like to thank my supervisor, Prof. Claudia Filippi, for guiding me during these four years. I have learned countless things from her about electronic structure, research in general, teaching, career choices. I am especially thankful for the scientific integrity and resilience I learned from her in face of (at times) negative results.

From people I have met in the university I would like to mention Omar, who has helped me settle in Enschede, the office, and quantum chemistry in general; Riccardo and Habib, my colleagues with whom we collaborated in ways work-related and otherwise-I wish you a successful continuation of your projects and a painless $\mathrm{PhD}$ defense! I would like to thank the members of our "sister group" CBP: Wim, Wouter, Nana, Vishal, Vishal (in no particular order), and Matteo, and past members thereof: Tom, Gabriel, Igor, Li, and Barry. The discussions we had at coffee breaks and other occasions truly put the "Ph" in this $\mathrm{PhD}$. Finally, I would like to thank Els, our secretary, who has helped with my endless paperwork with endless patience.

I am also indebted to co-authors from other universities: Ali Alavi, George Booth, and Simon Smart from Cambridge, Johannes Neugebauer and Carolin König from Münster, Adalgisa Sinicropi from Siena, Benedetta Mennucci from Pisa, Carles $\mathrm{Cu}-$ rutchet from Barcelona, André Severo Pereira Gomes from Lille, and Christoph Jacob from Braunschweig. It has been a privilege to work with and learn from them.

Outside work, Enschede allowed me to travel the world without having to go too far off de Oude Markt. I spent time (learning Dutch, P-NUT, football, volleyball, chess, and winning at PainStation, among other things) and had fun with so many different people that I dare not try to name them all and instead will mention their countries (apologies for missing some!): Australia, Azerbaijan, Belgium, Brazil, Bosnia, China, Colombia, Croatia, Czech Republic, Denmark, England, France, Finland, Germany, Ghana, Greece, India, Indonesia, Iran, Ireland, Italy, Kenya, Korea, Moldova, Nigeria, Pakistan, Poland, Romania, Russia, Scotland, Serbia, Spain (and Catalonia ;) ), Turkey, Ukraine, the United States, ...

Ik heb zo veel gezellige Nederlanders ontmoet in de universiteit, bij Max Euwe (schaken), de Boerderie (bridgen), en eigenlijk overal. Ik voelde me gelijk thuis in Nederland en ga nog zeker vaak terugkeren, Heidelberg is op een steenworp afstand! Bedankt iedereen voor de gastvrijheid, Nederlandse lessen, en alles!

Végü, de nem utolsó sorban hálásan köszönöm a családom támogatását minden szempontból. Nagyon sokat jelent az is, hogy eljöttetek Enschedébe a védésemre. Remélem, nem kaptok fehérjemérgezést! 


